\title{
AN INTERACTIVE DEBUGGING TOOL FOR C++ BASED ON DYNAMIC SLICING AND DICING
}

\author{
By \\ WINAI WICHAIPANITCH \\ Bachelor of Science (Electrical Engineering) \\ Rajamangala Institute of Technology \\ Bangkok, Thailand \\ 1984 \\ Master of Science (Computer Science) \\ Oklahoma State University \\ Stillwater, Oklahoma \\ 1992
}

Submitted to the Faculty of the

Graduate College of the Oklahoma State University

in partial fulfillment of the requirements for the Degree of DOCTOR OF PHILOSOPHY

August 2003 


\section{AN INTERACTIVE DEBUGGING TOOL FOR C++}

BASED ON DYNAMIC SLICING AND DICING

Thesis Approved:

$\frac{\text { Mansw M. Samadgaded }}{\text { Thesis Adviser }}$

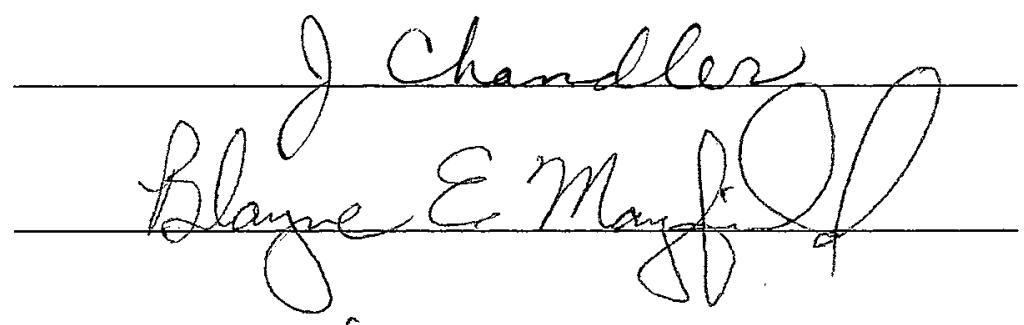

Eenrew. Dnagen

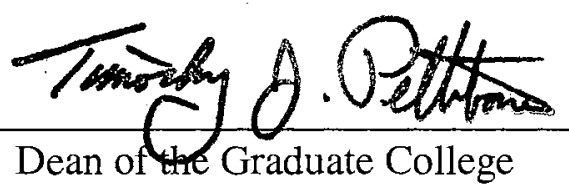




\section{PREFACE}

Since the article "Program Slicing" by Mark Weiser was initially published in 1981 [Weiser 81], program slicing has gained wide recognition in both academic and practical arenas. Several debugging tools have been developed that utilize program slicing. For example, Focus (designed and implemented by Lyle in 1984) was designed to be used with Fortran programs, and C-Sdicer (designed and implemented by Nanja and Samadzadeh in 1990) and C-Debug (designed and implemented by Wichaipanitch and Samadzadeh in 1992) were designed to be applicable to C language programs based on dynamic slicing.

Program slicing [Weiser 81,82 , and 84 ] is one of the debugging methods used to localize errors in a program. The idea of program slicing is to focus on the statements that have something to do with a certain variable of interest (criterion variable), with the unrelated statements being omitted. Using slicing, one obtains a new program of generally smaller size that still maintains all aspects of the original program's behavior with respect to the criterion variable. Dynamic slicing differs from static slicing in that it is defined on the basis of a computation or an execution rather than on all possible computations. Furthermore, it allows one to treat the elements and fields in dynamic records as individual variables [Korel and Laski 90]. As a result, the slice size computed based on the dynamic slicing technique is generally smaller. Moreover, 
dynamic slicing allows one to keep track of the run-time type binding (involving the type of each object) that is unknown at compile time but is determined when the program is executed. Dynamic slicing technique was used in this study.

Dicing technique [Lyle 84] [Nanja 90] [Nanja and Samadzadeh 90] can then be used to compare two or more slices resulting from the program slicing technique in order to identify the set of statements that are likely to contain an error. The formal model of static/dynamic slicing/dicing is presented. There is a need for debugging tools that are capable of making some deductions regarding the presence and location of errors in programs.

The main objective of this work was to develop an interactive debugging tool for $\mathrm{C}++$ programs. The tool that was developed is called $\mathrm{C}++$ Debug and it uses program slicing and dicing techniques. The design started by including simple statements first and then expanded to pointers, structures, functions, and classes. In order for $\mathrm{C}++\mathrm{Debug}$ to be more powerful, dynamic slicing rather than static slicing was chosen. The work includes new algorithms that handle Class, Function, and Pointer in C++. 


\section{ACKNOWLEDGMENTS}

I owe a great deal of gratitude and appreciation to my major adviser Dr. Mansur H. Samadzadeh for his guidance, motivation, dedication, and valuable instruction during my dissertation work. Dr. Samadzadeh continued to spend endless hours reviewing my work and offering suggestions for further refinement.

I wish to thank my other committee members Drs. Blayne E. Mayfield, John P. Chandler, and Cecil Dugger. Their time and efforts are greatly appreciated.

Many thanks are due to my wife Cholada for her moral support.

Finally, but certainly not least, I wish to thank my parents, Mr. Arun and Mrs. Tonghaw. It was their examples of hard work over many years that gave me the inspiration and motivation to complete my graduate studies. I will be forever indebted to them for this. 


\section{TABLE OF CONTENTS}

Chapter Page

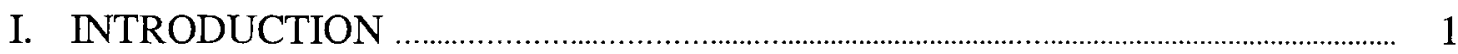

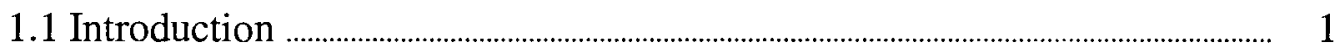

1.2 Purpose of the Study ............................................................................................... 2

1.3 Organization of the Report .............................................................................................. 3

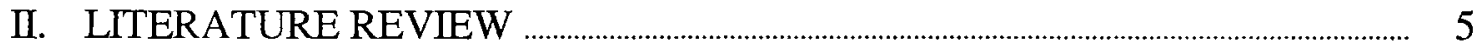

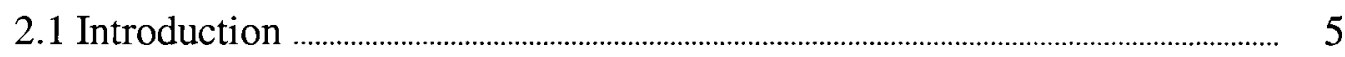

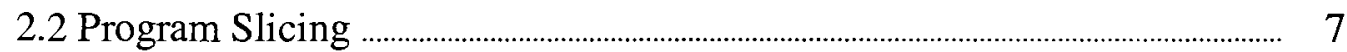

2.2.1 Static Slicing .............................................................................................. 8

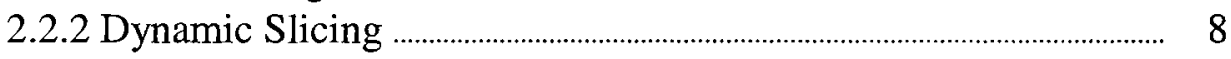

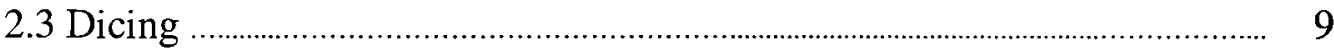

2.4 Examples ................................................................................................... 9

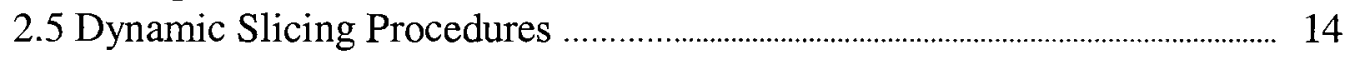

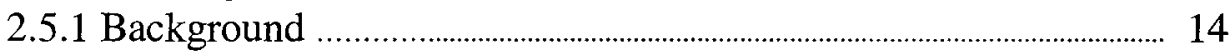

2.5.2 Slicing Criterion ........................................................................................... 16

2.5.3 Steps Needed to Obtain a Dynamic Program Slice ...................... 17

2.6 Dicing Procedures .................................................................................................. 24

2.7 Problems with Slices .............................................................................................. 25

III. C++ DYNAMIC SLICING AND DICING PROCEDURES .................................... 26

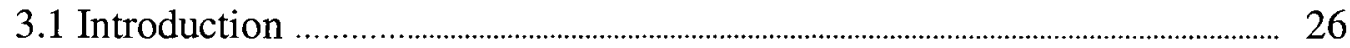

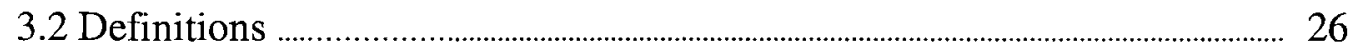

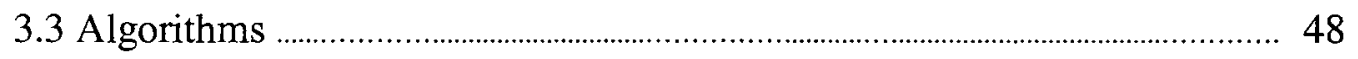

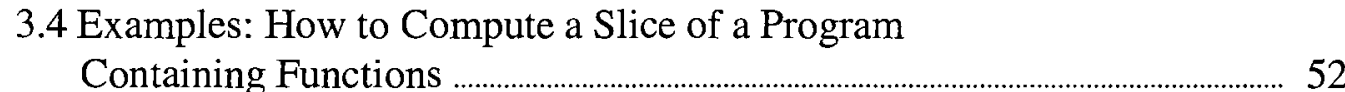

3.5 A Slice with Classes, Structures, and Unions ......................................................... 59

3.6 Problems and Situations in C++ That Were Taken into

Account in the Design ............................................................................................. 70

3.7 Dicing Procedure .................................................................................................... 72 


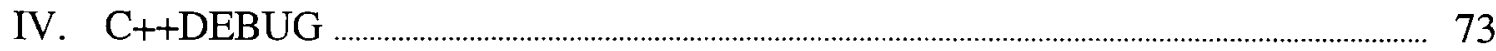

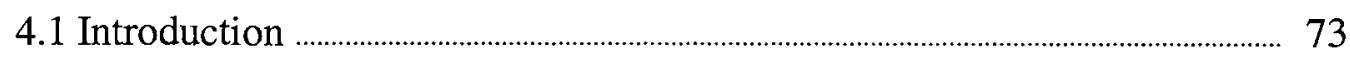

4.2 Software specification .................................................................................... 74

4.3 Software Design and Implementation .................................................................. 74

4.3.1 C++Debug Block Diagram ................................................................ 74

4.3.2 Datastructures .......................................................................................... 76

4.3.3 Symbol Tables ……….............................................................................. 76

4.4 Testing and Evaluation …......................................................................................... 77

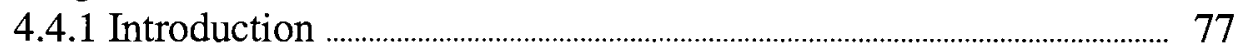

4.4.2 Testing .................................................................................................... 77

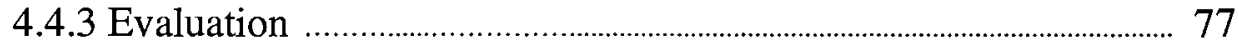

4.5 Limitations ....................................................................................................... 77

4.6 Program Documentation ................................................................................. 78

4.7 System Evolution ................................................................................................... 78

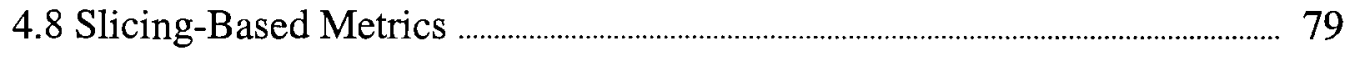

V. SUMMARY, CONCLUSIONS, AND FUTURE WORK …….................................. 82

5.1 Summary ……

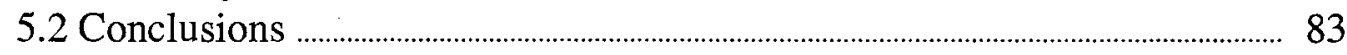

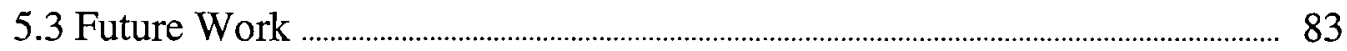

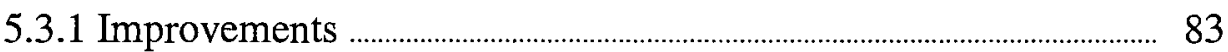

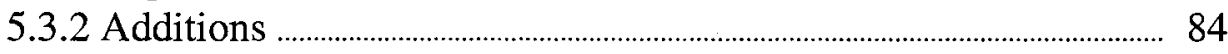

5.3.3 Future Work …………......................................................................................... 84

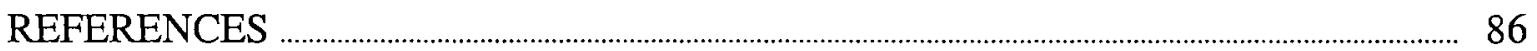

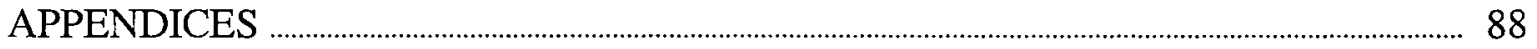

APPENDIX A - GLOSSARY ....................................................................... 89

APPENDIX B - USER'S MANUAL FOR C++DEBUG …........................................... 94

APPENDIX C - DATASTRUCTURES DESIGN FOR C++DEBUG BASED ON DYNAMIC PROGRAM SLICING AND DICING …………………………............ 104

APPENDIX D -SOFTWARE SPECIFICATIONS …………………………..... 120 
Chapter

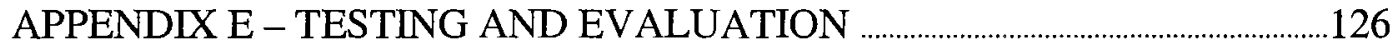

APPENDIX F - SAMPLE PROGRAMS USED FOR THE

COMPUTATION OF SLICING-BASED METRICS

APPENDIX G - SAMPLE C++DEBUG SOURCE CODE LISTING

146 


\section{LIST OF FIGURES}

Figure $\quad$ Page

1. The basic idea of program slicing and dicing .................................................................. 6

2. A program for counting occurrences and calculating the sum and average of a set of numbers

3. The output data of the program in Figure 2

4. A static program slice computed based on variable CountNumber in line 19 of the program in Figure 2

5. A dynamic program slice computed based on variable CountNumber [1] in line 19 of the program in Figure 2

6. A dynamic program slice computed based on variable CountNumber [2] in line 19 of the program in Figure 2

7. A program slice computed based on variable Sum in line 19 of the program in Figure 2

8. A program slice computed based on variable Avg in line 19 of the program in Figure 2

9. A final program segment after using dicing

10. A trajectory of the program from Figure 2 on input data MaxData $=2$, Data $=(3,5)$

11. The sets $D\left(X^{p}\right)$ and $U\left(X^{p}\right)$, definition and use, for the trajectory in Figure 10

12. The DU (definition-use) relation for the trajectory depicted in Figure 10 
13. The TC (test-control) relation for the trajectory depicted in Figure 10

14. The IR (identity relation) relation for the trajectory depicted in Figure 10

15. A dynamic program slice computed based on variable CountNumber[1] in line 19 of the program in Figure 2

16. A dynamic program slice computed based on variable CountNumber [5] in line 19 of the program in Figure 2

17. A dynamic program slice computed based on variable Sum in line 19 of the program in Figure 2

18. A dynamic program slice computed based on variable Avg in line 19 of the program in Figure 2

19. The final program segment after slicing and dicing 25

20. A program for computing the factorial of a number 28

21. A trajectory of the program in Figure 20 on input data $\mathrm{Num}=3$

22. The sets $\mathrm{M}\left(\mathrm{TF}_{\mathrm{Fac}}\right), D F_{\mathrm{Fac}}\left(\mathrm{X}^{\mathrm{P}}\right), \mathrm{UF}_{\mathrm{Fac}}\left(\mathrm{X}^{\mathrm{P}}\right)$, and $\mathrm{LF} \mathrm{F}_{\mathrm{Fac}}\left(\mathrm{X}^{\mathrm{P}}\right)$ for the trajectory in Figure 21

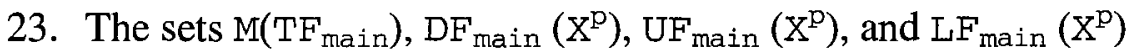
for the trajectory in Figure 21

24. The $D U F_{F a c}$ relation for the trajectory depicted in Figure 21 35

25. The $D U F_{\text {main }}$ relation for the trajectory depicted in Figure 21

26. The $L D R F_{F a c}$ relation for the trajectory depicted in Figure 21 35

27. The $L D R F_{\text {Main }}$ relation for the trajectory depicted in Figure 21 35

28. The $\mathrm{TCF}_{\mathrm{FaC}}$ relation for the trajectory depicted in Figure 21 
29. The $I R F_{\text {Fac }}$ relation for the trajectory depicted in Figure 21

30. A trajectory of functions $A$ and $B$ where function $A$ calls function $B$ 38

31. Illustrate Called-to-Calling 39

32. Illustrate Calling-to-Called 40

33. The EI relation for the trajectory depicted in Figure 21

34. The IE relation for the trajectory depicted in Figure 21

35. Rules for computing the CS (control scope) set

36. The Prototype, Called, Calling, D, U, DCL, VS, and CS sets for the program depicted in Figure 20

37. Algorithm to compute a set of slices

38. Slicing data structures 50

39. Algorithm to compute a slice of each function

40. Function to compute the scope of influences of a slice

41. A dynamic program slice computed based on variable Num in line 23 of the program in Figure 20

42. A dynamic program slice computed based on variable Fac in line 22 of the program in Figure 20 56

43. A dynamic program slice computed based on variable $I$ in line 8 of the program in Figure 20

44. A program for calculating the sum and average of a set of numbers 60

45. The trajectory of the program from Figure 44 on input data $\operatorname{Max}=4, \operatorname{Num}=(10.0,20.0,15.0,5.0)$

46. The Prototype, Called, Calling, D, U, DCL, VS, and CS sets for the program depicted in Figure 20 
47. The $D U F_{\text {Main }}, T C F_{\text {Main }} L D F_{\text {Main }}$, and $I R F_{\text {Main }}$ relations that are called by $32^{20}$ for the trajectory depicted in Figure 45

48. The DUF Compute, $T C F_{\text {Compute }} \mathrm{LDF}_{\text {Compute, }}$, and IRF $\mathrm{Compute}_{\text {relations }}$ that are called by $32^{20}$ for the trajectory depicted in Figure 45

49. The DUF Sum $_{\text {, }} \mathrm{TCF}_{\text {Sum }}, \mathrm{LDF}_{\text {Sum }}$, and $I R F_{\text {Sum }}$ relations that are called by $32^{20}$ for the trajectory depicted in Figure 45

50. The $\mathrm{DUF}_{\text {Sum }}, \mathrm{TCF}_{\mathrm{Sum}} \mathrm{LDF}_{\text {Sum }}$, and $I R F_{\text {Sum }}$ relations that are called by $24^{22}$ for the trajectory depicted in Figure 45

51. The $D U F_{A v g}, T C F_{A v g}$, and $I R F_{A v g}$ relations for the trajectory depicted in Figure 45

52. A dynamic program slice computed based on variable Avg in line 33 of the program in Figure 44

53. A dynamic program slice computed based on variable Sum in line 32 of the program in Figure 44

54. The final program segment after slicing and dicing 72

55. Block diagram of $\mathrm{C}++$ Debug 75

56. Help menu and prompt 95

57. The trajectory path 99

58. Data structure of Types .105

59. A C++ program that uses iterators 105

60. Show the database of Types used in $\mathrm{C}++$ .106

61. Show how the database stores Types of the program in Figure 59. 106

62. Data structure of Declarations 107

63. Show how the database stores Declarations of the program in Figure 59 
Figure

64. Scopes of variable $\mathrm{x}$ as a global, local, and second local ….......................................... 108

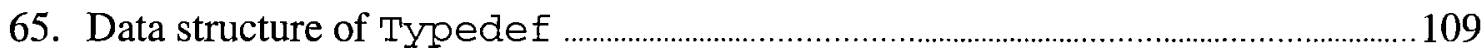

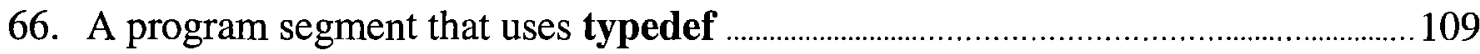

67. Show how the database stores Typedef defined by typedef in Figure 66

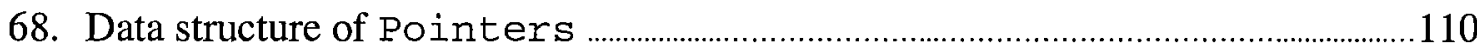

69. A program segment that uses pointers ………………............................................. 110

70. Show how the database stores Pointers of the part of the program in Figure 69

71. Show how the database stores $\operatorname{De} f(n)$ and $\operatorname{Re}(n)$ of the part of the program in Figure 69

72. Data structure of Arrays

73. A program segment that uses arrays

74. Show the data base of Arrays used by the part of the program in Figure 73

75. A program segment that uses pointers into arrays

76. Show how the database uses function InsertPointerName(ID) in Section C.3.1 to store variables of the part of the program in Figure 75

77. Show how the database uses function InsertArrayName(ID) in Section C.3.2 to store variables of the part of the program in Figure 75

78. Show how the database stores $\operatorname{Def}(\mathrm{n})$ and $\operatorname{Ref}(\mathrm{n})$ of the part of the program in Figure 75

79. A program segment that uses const

80. Show how the database stores constant declared in Figure 79 
81. Show how the database uses function InsertPointerName(ID) in Section C.3.1 to variables of the part of the program in Figure 79

82. Show how the database stores $\operatorname{Def}(n)$ and $\operatorname{Re} f(n)$ of the part of the program in Figure 79

83. Data structure of References

84. A program segment that uses references

85. Show how the database uses function InsertReferenceName(ID) to store variables of the part of the program in Figure 84

86. A program segment that uses pointer to void

87. Show how the database uses function InsertPointerName(ID) in Section C.3.1 to store variables of the part of the program in Figure 86

88. Data structure of Structures

89. A program segment that uses structures

90. Show how the database stores Structures of the part of the program in Figure 89

91. Show how to determine the set of variables by using functions $\operatorname{Def}(\mathrm{n})$ and $\operatorname{Def}(\mathrm{n})$

92. Part of function UsedVariable and its path 130

93. $\mathrm{C}++$ Debug block diagram 


\section{LIST OF TABLES}

Table

I. Description of the five test programs

II. Slicing-based metrics obtained from C-Sdicer

for the five test programs

III. Slicing-based metrics obtained from $\mathrm{C}++$ Debug

for the five test programs

IV. Background summary

V. Language frequency

VI. Slices and program changes

VII. Time measures for debugging by using the tool

VIII. Time measures debugging without using the tool 138 


\section{CHAPTER I}

\section{INTRODUCTION}

\subsection{Introduction}

Once a programmer finds that a program fails to function properly in the testing process, debugging techniques are used to localize the causes of the errors and to correct

them. All too often, one finds that the cost associated with testing and correcting a program is likely to increase as the size of the program increases and as the program becomes more complicated [Tassel 74]. As a result, various tools and methods have been developed to debug programs; for example, file printing utilities, module testing packages, built-in language facilities and programmed-in aids, post-mortem dumps, and source code amendment facilities [Tassel 74].

Program slicing [Weiser 81,82 , and 84 ] is one of the debugging methods used to localize errors in a program. The idea of program slicing is to focus on the statements that have something to do with a variable of interest (criterion variable), with the statement that are unrelated being omitted. Using the slicing method, one obtains a new program of generally smaller size, which still maintains all aspects of the original program's behavior with respect to the criterion variable. A dicing technique [Lyle 84] [Nanja 90] [Nanja and Samadzadeh 90] can then be used to compare two or more slices, 
resulting from the program slicing technique, to identify the set of statements that are likely to contain an error.

Program slicing can be classified into two main categories according to how slices are computed: static slicing and dynamic slicing. Static slicing is a method of computing program slices directly from the original source programs. Dynamic slicing is a method used to compute program slices from the trajectory, which is a feasible path that has actually been executed for some input. Dynamic slicing differs from static slicing in that it is defined on the basis of one computation rather than for all possible computations [Korel and Laski 90]. As the results, the slice size computed based on the dynamic slicing technique is typically smaller. Furthermore, it allows us to treat the elements and fields in dynamic records as individual variables.

$\mathrm{C}++$ is a general-purpose programming language and is successfully used in many application areas [Stroustrup 97]. Implementations of $\mathrm{C}++$ exist from some of the most modest microcomputers to the largest supercomputers, and for almost all operating systems. $\mathrm{C}++$ adds to $\mathrm{C}$ the concept of class, a mechanism for providing user-defined types that is also called abstract data type [Pohl 94]. C++ supports object-oriented programming by providing inheritance and run-time type binding in addition to the concept of class. As a result, a lot of programmers use $\mathrm{C}++$ to implement programs and hence tools are needed to localize the causes of errors detected during testing.

\subsection{Purpose of the Study}

The objective was to create an interactive debugging tool, called $\mathrm{C}++$ Debug, for debugging a $\mathrm{C}++$ program running under UNIX on the SUN machine in the Computer 
Science Department at OSU. C++Debug was designed to function as a utility program of the UNIX system and was developed based on slicing and dicing techniques. It was designed in a way to provide ease of use and convenience on the part of the user. Using $\mathrm{C}++$ Debug, a user can interact with the computer in locating errors in a program. In order for $\mathrm{C}++$ Debug to give smaller slice sizes, dynamic slicing rather than static slicing was chosen.

The scope of $\mathrm{C}++$ Debug includes programs that contain ANSI $\mathrm{C}$ and $\mathrm{C}++$ codes. Classes and objects, unions, records, arrays, pointers, references, dynamic allocations, function and operator overloading, copy constructors and defaults, inheritances, virtual functions and polymorphism, templates, and exception handling were included also.

\subsection{Organization of the Report}

The rest of this dissertation report is organized as follows. Chapter II reviews the literature related to general information on program slicing and dicing techniques. The chapter concludes with a discussion of the advantages and disadvantages of dynamic and static slicing, and the procedures used to locate errors in a program using dynamic slicing and dicing techniques. Chapter III presents definitions and algorithms to get slices and dices in a $\mathrm{C}++$ program. Chapter IV presents the steps involved in the design and implementation of $\mathrm{C}++$ Debug, its testing and evaluation, and the advantages and limitations of C++Debug. Chapter V contains a summary, conclusions, and some areas of future work.

There are seven appendices: one on notation, one containing a user's manual for $\mathrm{C}++$ Debug, one containing datastructure design for $\mathrm{C}++$ Debug, one containing software 
specifications, one containing testing and evaluation, one containing sample programs used for the computation of slicing-based metrics, and the final appendix contains sample source code listing of $\mathrm{C}++$ Debug. 


\section{CHAPTER II}

\section{LITERATURE REVIEW}

\subsection{Introduction}

Localizing program errors is an arduous and time-consuming task, especially when programs written by other people are involved. Several attempts have been made to find ways that can enable one to locate errors more rapidly and effectively. Program slicing [Weiser 81, 82, and 84] [Korel 88] [Gallagher and Lyle 91] is one of several methods that have been used for this purpose.

Figure 1 shows the basic idea of program slicing and dicing. Let us assume that Figure 1(a) is a program to compute a tax fee. It is a large program with, say, 9000 lines of code. In fact, the actual number of statements or functions is not very important. For this program, let us assume we have found that the variable avg in line 8700 gives an incorrect result, e.g., 4.25 instead of 3.25. Because the program is too large, it is difficult to localize where the error is. Using program slicing technique based on variable avg, we can get a new program of smaller size, 15 lines in this case, which still maintains all aspects of the original program's behavior (Figure 1(b)). Now, although the result is a new program of a smaller size, sometime we cannot find where the error is. Fortunately, 


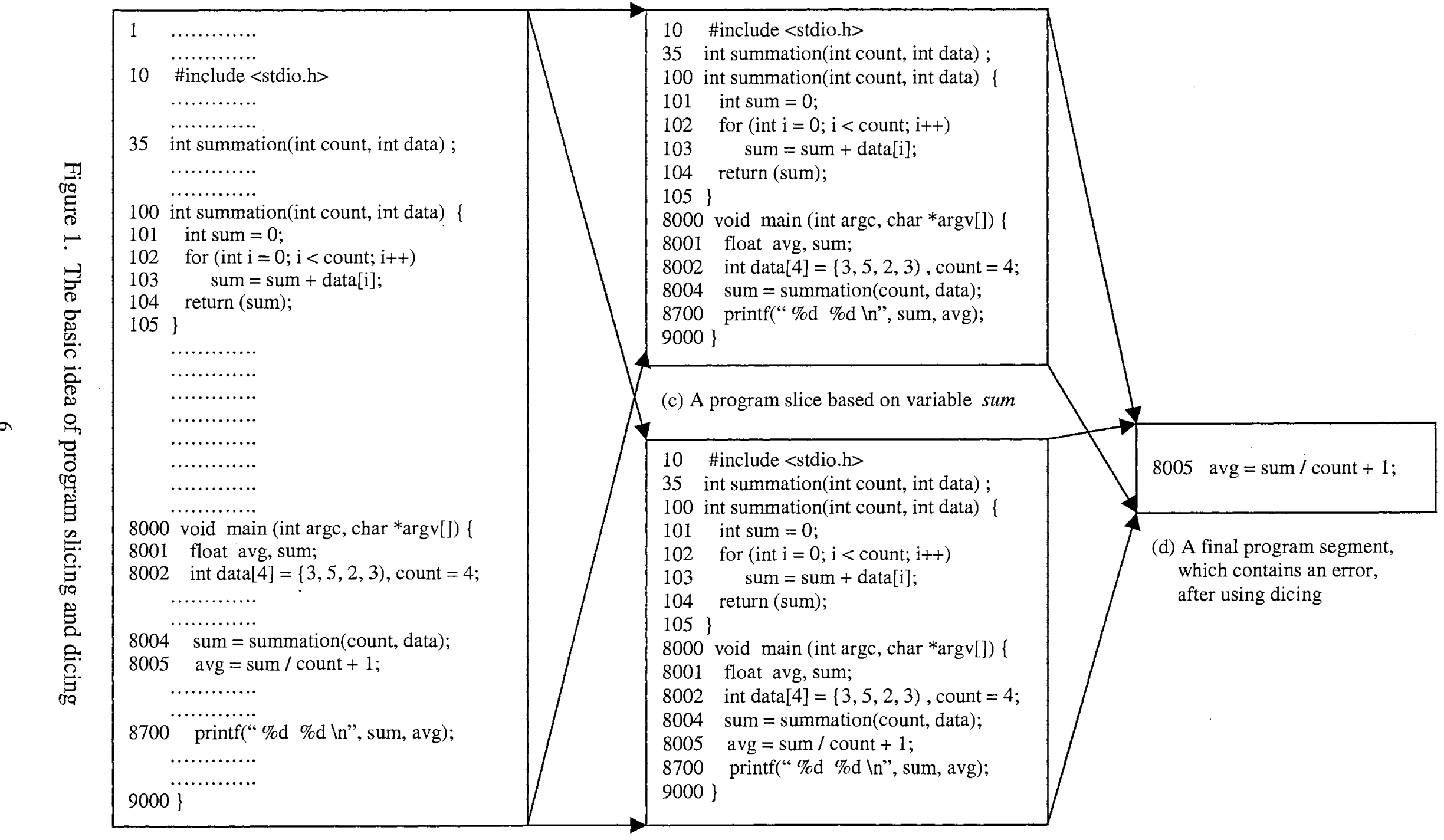

(a) Original program

(b) A program slice based on variable avg 
in this example, variable sum in line 8700 gives the correct answer and yields the slice as shown in Figure 1(c). To localize an error, dicing technique can be used by comparing both slices, where one contains no errors but the other one does. Some statements sliced on correct variables will then be removed from those sliced on variables with incorrect values. Upon completion of these steps, we get a new slice that is smaller and contains an error as shown in Figure 1(d). Finally, it is discovered that the correct statement should be avg $=$ sum $/$ count, instead of avg $=$ sum $/$ count +1 .

\subsection{Program Slicing}

The notion of slicing is based on the premise that instead of locating errors in the original program, which can be of large size, one can locate errors in a program of smaller size, which is sliced from the original program but still preserves part of the original program's behavior for a particular variable [Weiser 84].

Advantages of slices and slicing are based on four points [Weiser 84]. First, slices can be found automatically by a method used to decompose programs through analyzing their data flow and control flow. Second, a slice is normally smaller than the original program. As a consequence, when slicing at a variable of interest, the size of the resulting program slice is generally smaller than that of the original program. Third, slices can be executed independently of one another. In other words, a slice is itself an executable program whose behavior is identical to the specified subset of the original program's behavior. In other words, a slice produces a specific projection of the original program's behavior.

In addition to the four points mentioned above, Weiser also mentioned two 
intuitively desirable properties of a slice [Weiser 81]. First, a slice must be obtained from the original program by statement deletion. Second, once the statement deletion has been accomplished, the behavior of the resulting slice must correspond to the behavior of the original program as observed through a particular variable in the slicing criterion.

Program slicing can be classified into two main categories: static slicing and dynamic slicing. These categories are discussed below.

\subsubsection{Static Slicing}

Static slicing [Weiser 81,82 , and 84 ] is a method defined on the basis of all computations of a program. It yields a program slice of generally larger size than that of dynamic slicing (or, in the best case, of equal size to that of dynamic slicing), because static slicing often gives a slice containing statements that have no influence on the values of variables of interest for a particular execution [Korel and Laski 90]. Also, static slicing cannot treat the array elements and fields in dynamic records as individual variables. Finally, static slicing cannot support run-time handling. A static program slice is determined directly from the original source program (see Section 2.4 for examples).

\subsubsection{Dynamic Slicing}

Unlike static slicing, dynamic slicing [Korel 88] [Korel and Laski 88 and 90] is defined on the basis of one computation rather than all computations, and generates a dynamic program slice by computing from the trajectory that is a feasible path that has actually been executed for some input of the original source program (see Section 2.4 for examples). In addition, this method enables one to treat the array elements and fields in dynamic records as individual variables [Korel and Laski 90]. In this way, the size of the 
resulting slice becomes generally smaller. Moreover, dynamic slicing allows one to keep track of run-time type binding, which is unknown at compile time but is determined until when the program is executed.

\subsection{Dicing}

Dicing is the process of identifying a set of statements likely to contain an error [Lyle 84] [Nanja 90] [Nanja and Samadzadeh 90] [Samadzadeh and Wichaipanitch 93]. The idea is first to compare two or more slices using program slicing techniques (see Section 2.4 for an example). Only one of these will slice on a variable with an incorrect value and the other(s) will slice on variables with correct values. One must first ascertain that the latter contains no errors. Some statements sliced on correct variables will then be removed from those sliced on the variable with incorrect values. Upon completion of these steps, a new slice is obtained that is smaller and contains the error.

It should be noted that the validity of the use of dicing rests on three important assumptions [Weiser and Lyle 86]. First, it is necessary that testing be reliable and that all incorrectly computed variables be identified. Second, if the computation of a variable $\mathrm{V}$ depends on the computation of another variable $\mathrm{W}$, then whenever $\mathrm{W}$ has an incorrect value, so does $\mathrm{V}$. Third, it is necessary that one and only one fault exist in the program.

The next sections provide examples of the computation of slices (static and dynamic) and dices.

\subsection{Examples}

Illustrated below are the comparison of the sizes of program slices generated from 
static slicing, dynamic slicing, and dicing techniques.

The program in Figure 2 is designed to count the number of individual integers falling between 1 and 5 read from the input data. Furthermore, this program computes the sum and average of these integers. In this example, the variable MaxData is 5 and the array called Data contains 3,5,5,2, and 2. Upon completion of program execution, the program should yield the results as shown in Figure 3. However, this program

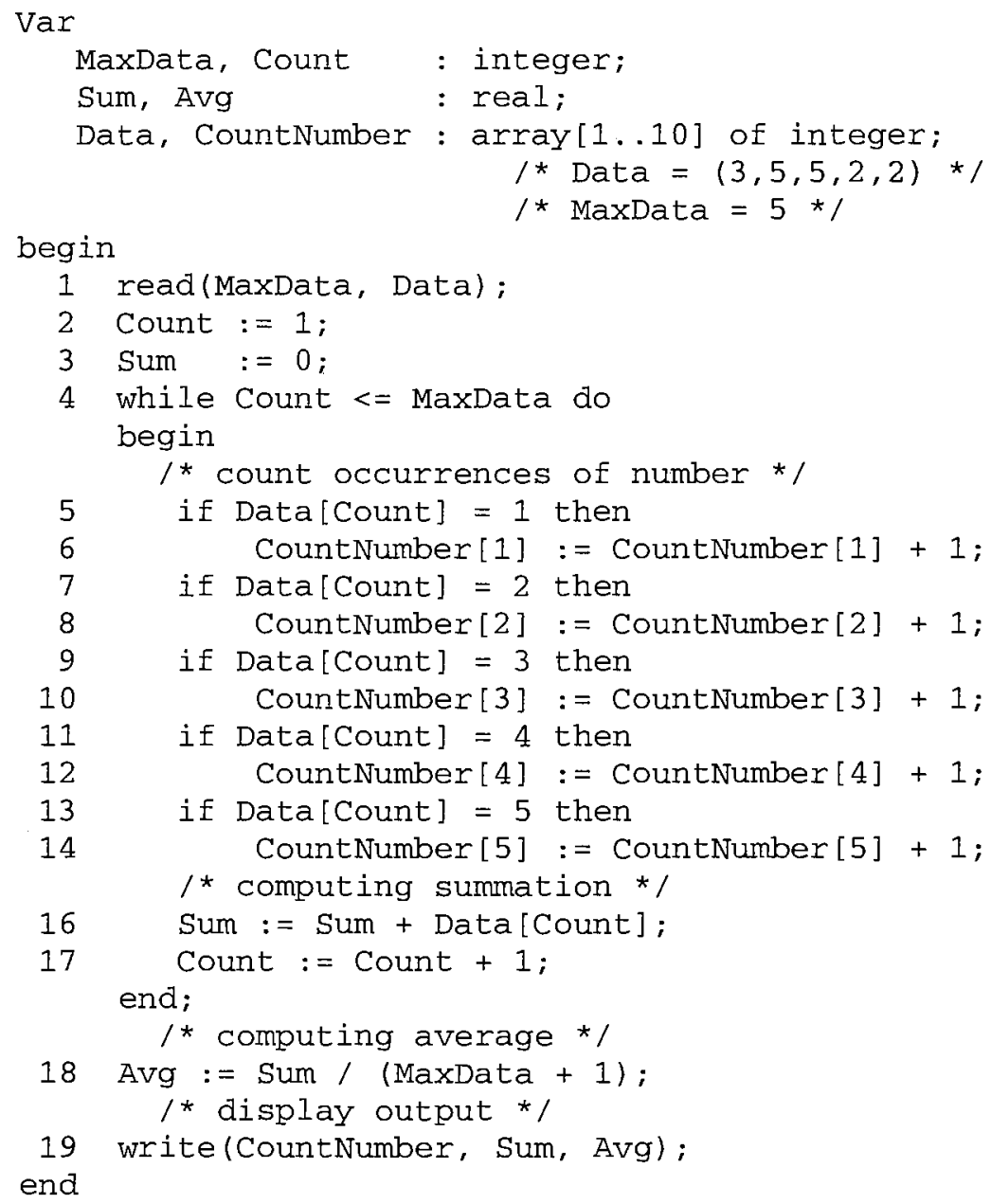

Figure 2. A program for counting occurrences and calculating the sum and average of a set of numbers 
contains an error in line 18. Rather than Avg := Sum/MaxData, the program computes Avg := Sum/MaxData +1$)$, thus yielding an error $($ Avg $=2.8$ instead of 3.4). To localize such an error, program slicing and dicing techniques can be used.

Figure 4 is a static program slice computed based on variable CountNumber in line 19. The static slicing method treats array variables as a single variable regardless of the number of elements in the array. In contrast, if the dynamic slicing approach is used, array elements are treated as individual variables. As a result, the size of a program slice is generally reduced by using dynamic slicing techniques. As shown in Figure 5, no

Number of each integer: $0,2,1,0$, and 2, respectively Sum $=17$

$\operatorname{Avg}=3.4$

Figure 3. The output data of the program in Figure 2

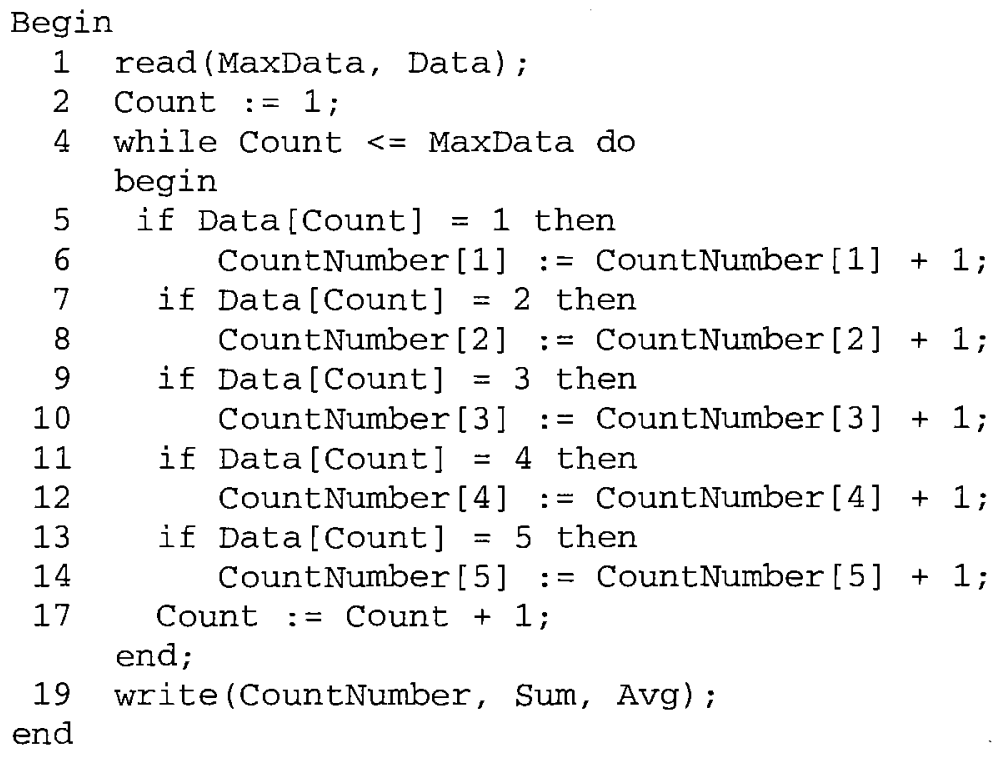

Figure 4. A static program slice computed based on variable CountNumber in line 19 of the program in Figure 2 
program slice results from variable CountNumber [1] in line 19.

Figure 6 shows the program slice resulting from computing a dynamic slice based on variable CountNumber [2] in line 19. Obviously, the sizes of the program slices resulting from CountNumber[1] and CountNumber[2] are different because dynamic slicing treats the two array elements as two different variables whereas static slicing does not.

Figures 7 and 8 depict program slices generated based on variables Sum and Avg, respectively, in line 19. For these variables, the static slicing method [Lyle 84] [Nanja 90] [Nanja and Samadzadeh 90] and the dynamic slicing method [Korel and Laski 90] yield identical results.

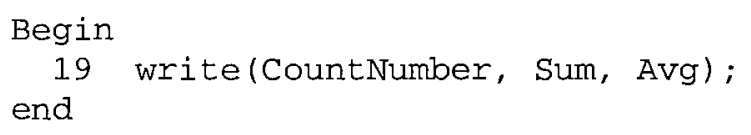

Figure 5. A dynamic program slice computed based on variable CountNumber [1] in line 19 of the program in Figure 2

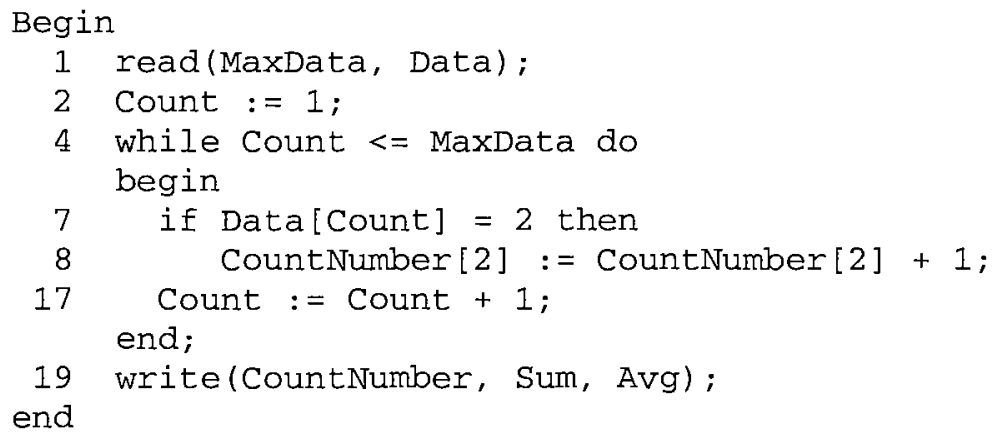

Figure 6. A dynamic program slice computed based on variable CountNumber [2] in line 19 of the program in Figure 2 
With the use of the dicing algorithm, a new program segment can be generated, as shown in Figure 9. This program segment contains the final erroneous line (which is line 18).

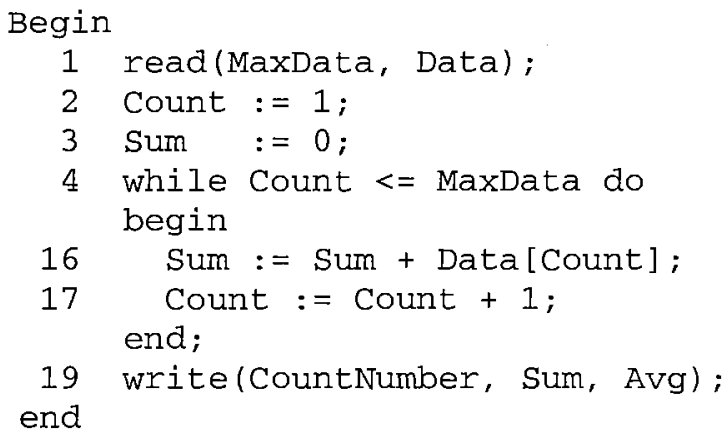

Figure 7. A program slice computed based on variable Sum in line 19 of the program in Figure 2

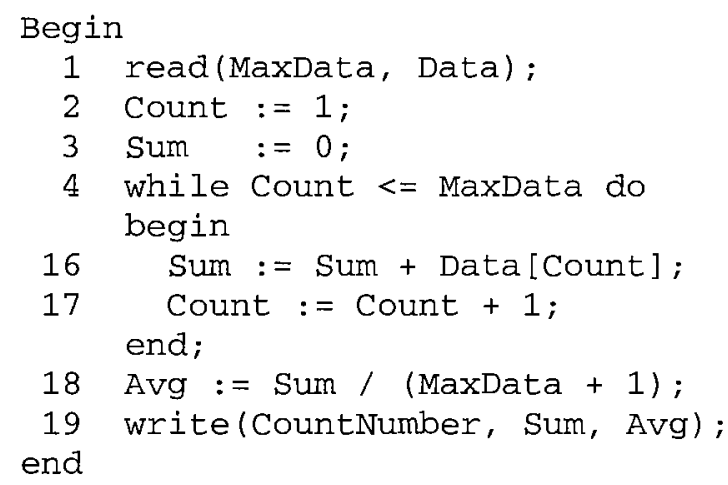

Figure 8. A program slice computed based on variable Avg in line 19 of the program in Figure 2

18 Avg := Sum / (MaxData + 1);

Figure 9. A final program segment after using dicing 


\subsection{Dynamic Slicing Procedures}

\subsubsection{Background}

To facilitate better understanding of program slicing, it is necessary that the following background [Korel and Laski 90] be presented.

Let the flowgraph of a program $\mathrm{P}$ be a directed graph $(\mathrm{N}, \mathrm{A}, \mathrm{s}, \mathrm{e})$ and $\mathrm{C}$ be a slicing criterion, where $\mathrm{N}$ is the set of nodes, $\mathrm{A}$ is a binary relation on $\mathrm{N}$ (a subset of $\mathrm{N} \mathrm{x}$ $N)$, referred to as the set of arcs, $s \in N$ is a unique entry node, and $e \in N$ is a unique exit node.

Each node in $\mathrm{N}$ consists of one statement: a single instruction or a control instruction. A single instruction can be an assignment statement, an input or output statement, etc. A control instruction can be such statements as an if-then-else statement or a while statement that are also called test instructions.

An $\operatorname{arc}(n, m) \in A$ corresponds to a possible transfer of control from instruction $n$ to instruction $\mathrm{m}$.

A path from the entry node $s$ to some node $k, k \in N$, is called a sequence $<n_{1}, n_{2}$, $\ldots, n_{q}>$ of instructions, such that $n_{1}=s, n_{q}=k$, and $\left(n_{i}, n_{i+1}\right) \in A$, for all $n_{i}, 1 \leq i<q$. If there are input data, which cause a path to be traversed during program execution, the path is feasible.

A trajectory is a feasible path that has actually been executed for some input. For example, in Figure $10,<1,2,3,4,9,10,16,17,4,13,14,16,17,4,18,19>$ is the trajectory when the program in Figure 2 is executed on input data MaxData $=2$, Data $=$ $(3,5)$. A resulting trajectory can be the initial segment of an infinite path if the execution does not terminate in the case of an infinite loop. A trajectory is illustrated in terms of an 


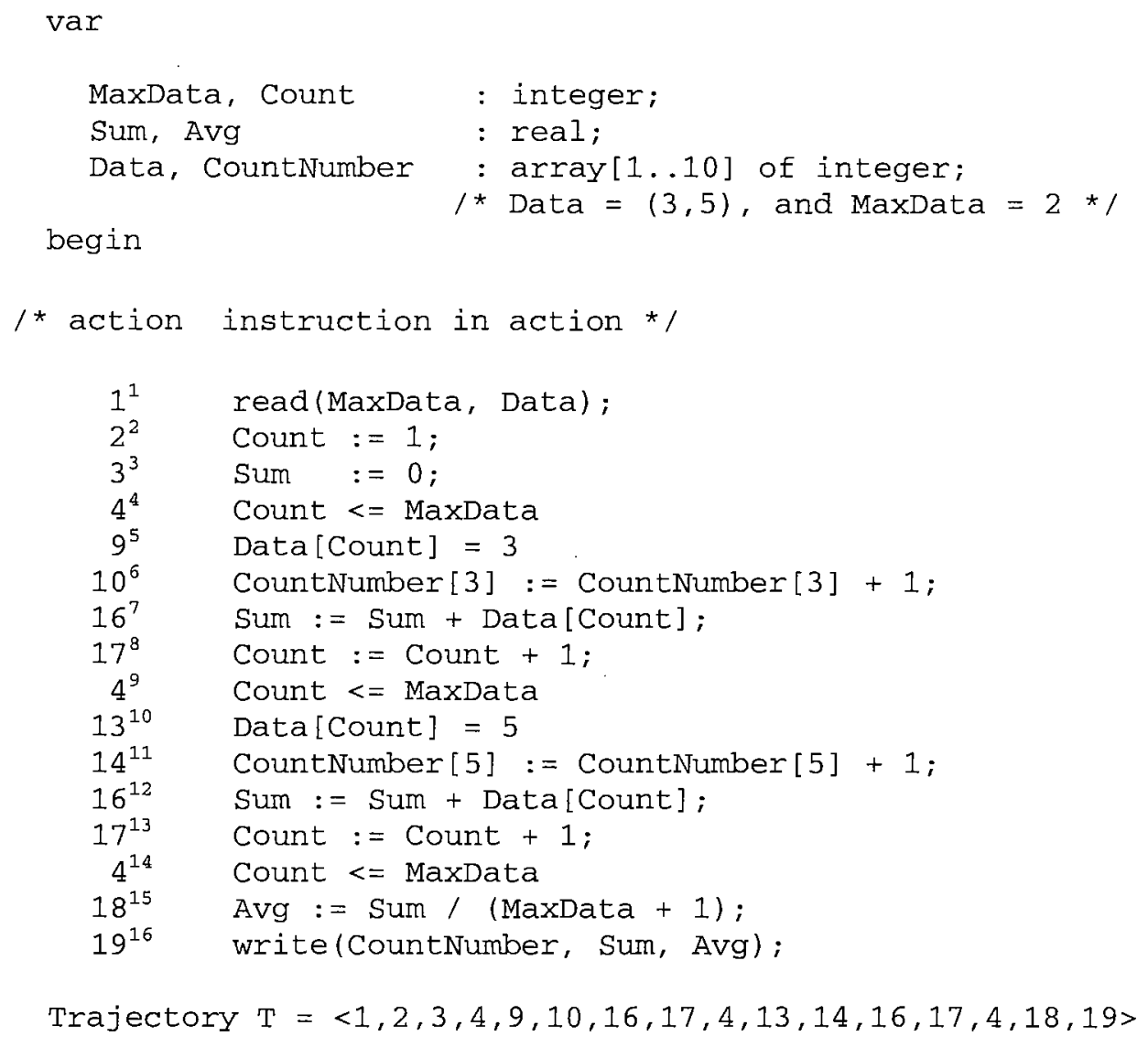

Figure 10. A trajectory of the program from Figure 2 on input data MaxData $=2$, Data $=(3,5)$

abstract list whose elements are accessed according to their positions in it. For example, $\mathrm{T}(2)=2$ and $\mathrm{T}(5)=9$. A trajectory is also illustrated in terms of a pair (instruction, its position in the trajectory), rather than the instruction itself, so as to distinguish between multiple occurrences of the same instruction in the trajectory.

For example, instruction $\mathrm{X}$ at position $\mathrm{p}$ in trajectory $\mathrm{T}$ is represented by pair $(\mathrm{X}$, p). For brevity and ease of understanding, pair $(\mathrm{x}, \mathrm{p})$ is replaced by $\mathrm{x}^{\mathrm{p}}$ and is referred to as an action. For example, $4^{4}$ and $4^{9}$ in trajectory $\mathrm{T}$ in Figure 10 are actions that involve the same instruction 4. An action $\mathrm{X}^{\mathrm{p}}$ is a test action if $\mathrm{X}$ is a test instruction. For 
example, $4^{4}, 4^{9}$, and $4^{14}$ in trajectory $\mathrm{T}$ in Figure 10 are test actions.

Let $\mathrm{T}=\left\langle\mathrm{X}_{1}, \mathrm{X}_{2}, \ldots, \mathrm{X}_{\mathrm{m}}>\right.$ denote a trajectory of length $\mathrm{m}$, and $\mathrm{q}$ be a position in $\mathrm{T}, 1$ $\leq q \leq \mathrm{m}$. Then the following can be obtained.

1. Front $(\mathrm{T}, \mathrm{q})$ denotes the sublist $\left\langle\mathrm{X}_{1}, \mathrm{X}_{2}, \ldots, \mathrm{X}_{\mathrm{q}}\right\rangle$, consisting of the first q elements of $\mathrm{T}$.

2. $\operatorname{Back}(\mathrm{T}, \mathrm{q})$ denotes the sublist $\left\langle\mathrm{X}_{\mathrm{q}+1}, \mathrm{X}_{\mathrm{q}+2}, \ldots, \mathrm{X}_{\mathrm{m}}\right\rangle$, consisting of elements that follows $\mathrm{T}(\mathrm{q})$, a trajectory at position $\mathrm{q}$.

So, for all $\mathrm{T}$ and $\mathrm{q}$ the following can be obtained. $\mathrm{T}=\operatorname{Front}(\mathrm{T}, \mathrm{q}) \| \operatorname{Back}(\mathrm{T}, \mathrm{q})$, where $\|$ represents concatenation.

3. $\mathrm{DEL}(\mathrm{T}, \mathrm{R})$, where $\mathrm{R}$ is a predicate on the set of instructions in $\mathrm{T}$, means a subtrajectory obtained from $\mathrm{T}$ by deleting from it all elements $\mathrm{T}(\mathrm{i})$ that satisfy $R$.

\subsubsection{Slicing Criterion}

A slicing criterion is the specification for a particular behavior of interest. A slicing criterion can be expressed as the values of some set of variables at some set of statements [Weiser 81]. If we let $\mathrm{T}$ be the trajectory of program $\mathrm{P}$ on input $\mathrm{x}$, a slicing criterion of program $P$ executed on $x$ can be defined as a triple $C=\left(x, I^{q}, V\right)$ where $I^{q}$ is an action in $\mathrm{T}$ and $\mathrm{V}$ is a subset of the variables in $\mathrm{P}$ [Korel and Laski 90].

It is readily apparent that the slicing criterion of dynamic slicing differs from that of static slicing. The slicing criterion of dynamic slicing contains an input value $\mathrm{x}$, whereas that of static slicing contains only a pair $\mathrm{C}=(I, V)$. This is because a change in the value of input $\mathrm{x}$ will result in a change in the trajectory, which in turn may result in a 
change in the size of the resulting slice. That is to say, the slicing criterion of dynamic slicing is defined in terms of a given trajectory on a specific input $\mathrm{x}$, rather than in terms of the set of all possible paths. In the case of static slicing, a slicing criterion is an instruction I in a program $P$, while in the case of dynamic slicing, a slicing criterion is an instruction $I$ at a particular execution position $q$ in a trajectory $\mathrm{T}$.

\subsubsection{Steps Needed to Obtain a Dynamic Program Slice}

The procedure needed to obtain a dynamic program slice can be summarized in five steps as explained below along with examples to illustrate the process.

1. Find a trajectory [Korel 88] [Korel and Laski 88 and 90] of the program (a trajectory is a feasible path traversed during program execution, see Subsection 2.5.1 for details). For the program in Figure 2, a trajectory is shown in Figure 10. In Figure 10, all instructions in the trajectory represent a pair consisting of an instruction and its position in the trajectory, instead of the instruction itself. In other words, $\mathrm{X}$ at position $\mathrm{p}$ in $\mathrm{T}$ will be referred to as pair $(\mathrm{X}, \mathrm{p})$ or $\mathrm{X}^{\mathrm{p}}$, which is referred to as an action [Korel and Laski 90 ]. For instance, $4^{4}$ and $4^{9}$ in trajectory $\mathrm{T}$ in Figure 10 are actions involving the same instruction 4. An action $\mathrm{X}^{\mathrm{p}}$ is a test action provided that $\mathrm{X}$ is a test instruction [Korel and Laski 90].

2. For each line $\mathrm{X}^{\mathrm{p}}$ in the trajectory, compute $\mathrm{U}\left(\mathrm{X}^{\mathrm{p}}\right)$, the set of variables that are used in $\mathrm{X}^{\mathrm{p}}$, and also compute $\mathrm{D}\left(\mathrm{X}^{\mathrm{p}}\right)$, the set of variables that are defined in $\mathrm{X}^{\mathrm{P}}$ [Korel and Laski 90]. For example, in the execution trace of Figure 10 we have

$$
\left.18^{15} \mathrm{Avg}:=\text { Sum / (MaxData }+1\right) \text {; }
$$

Avg is a set of variables defined in $18^{5}, \mathrm{D}\left(18^{5}\right)$. Sum and MaxData are a set of 
variables that are used in $18^{5}, U\left(18^{5}\right)$. The sets $U\left(X^{\mathrm{p}}\right)$ and $\mathrm{D}\left(\mathrm{X}^{\mathrm{p}}\right)$ for the trajectory in Figure 10 are shown in Figure 11.

\begin{tabular}{|c|l|l|}
\hline Action & \multicolumn{1}{|c|}{$\mathrm{D}\left(\mathrm{X}^{\mathrm{P}}\right)$} & $\mathrm{U}\left(\mathrm{X}^{\mathrm{D}}\right)$ \\
\hline $1^{1}$ & NaxData, Data & \\
$2^{2}$ & Count & \\
$3^{3}$ & Sum & Count, MaxData \\
$4^{4}$ & & Data [1], Count \\
$9^{5}$ & CountNumber [3] & CountNumber [3] \\
$10^{6}$ & Sum & Sum, Data [1], Count \\
$16^{7}$ & Count & Count \\
$17^{8}$ & Count, MaxData \\
$4^{9}$ & Data [2], Count \\
$13^{10}$ & CountNumber [5] & CountNumber [5] \\
$14^{11}$ & Sum, Data [2], Count \\
$16^{13}$ & Count & Count \\
$4^{14}$ & Count, MaxData \\
$18^{15}$ & Avg & Sum, MaxData \\
& & CountNumber, Sum, Avg \\
\hline
\end{tabular}

Figure 11. The sets $\mathrm{D}\left(\mathrm{X}^{\mathrm{D}}\right)$ and $\mathrm{U}\left(\mathrm{X}^{\mathrm{P}}\right)$, definition and use, for the trajectory in Figure 10

3. Compute the DU (Definition-Use) Relation, a relation in which one action assigns a value to an item of data and the other action uses that value [Korel and Laski 90]. For example, in the execution trace of Figure $11,2^{2}$ defines the variable Count, and $4^{4}, 9^{5}$, $16^{7}$, and $17^{8}$ use the defined value of that variable. Let $M(T)$ be a set of actions in a given trajectory $\mathrm{T}$, where $\mathrm{M}(\mathrm{T})=\{(\mathrm{X}, \mathrm{p}): \mathrm{T}(\mathrm{p})=\mathrm{X}\}$. DU is a binary relation on $\mathrm{M}(\mathrm{T})$ defined bellow [Korel 88]. 
$\mathrm{X}^{\mathrm{p}} \mathrm{DU} \mathrm{Y}^{\mathrm{t}}, 1 \leq \mathrm{p}<\mathrm{t}$, iff there exists a variable $\mathrm{v}$ such that

(1) $\mathrm{v} \in \mathrm{U}\left(\mathrm{Y}^{\mathrm{t}}\right)$, and

(2) $\mathrm{X}^{\mathrm{P}}$ is the last definition of $\mathrm{v}$ at $\mathrm{t}$

where, the last definition $\mathrm{X}^{\mathrm{p}}$ of variable $\mathrm{v}$ at $t$ is the action which last assigned a value to $\mathrm{v}$ when $\mathrm{t}$ was reached on trajectory $\mathrm{T}$.

For example, in the trajectory of Figure $11,2^{2}$ is the last definition of variable Count at the execution positions 3 through 8 . The DU Relation for the trajectory in Figure 11 is shown in Figure 12.

$$
\begin{aligned}
& \left.\mathrm{DU}\left(1^{1}\right)=\quad=\quad 4^{4}, 9^{5}, 16^{7}, 4^{9}, 13^{10}, 16^{12}, 4^{14}, 18^{15}\right\} \\
& \mathrm{DU}\left(2^{2}\right)=\left\{4^{4}, 9^{5}, 16^{7}, 17^{8}\right\} \\
& \mathrm{DU}\left(3^{3}\right)=\left\{16^{7}\right\} \\
& \mathrm{DU}\left(10^{6}\right)=\left\{19^{16}\right\} \\
& \mathrm{DU}\left(16^{7}\right)=\left\{16^{12}\right\} \\
& \mathrm{DU}\left(17^{8}\right)=\left\{4^{9}, 13^{10}, 16^{12}, 17^{13}\right\} \\
& \mathrm{DU}\left(14^{11}\right)=\left\{19^{16}\right\} \\
& \mathrm{DU}\left(16^{12}\right)=\left\{18^{15}, 19^{16}\right\} \\
& \mathrm{DU}\left(17^{13}\right)=\left\{4^{14}\right\} \\
& \operatorname{DU}\left(18^{15}\right)=\left\{19^{16}\right\}
\end{aligned}
$$

Figure 12. The DU (definition-use) relation for the trajectory depicted in Figure 10

4. Compute the TC (Test-Control) Relation, capturing the effect between test actions and actions that have been chosen to execute by those test actions [Korel and Laski 90]. For example, in the execution trace of Figure 10, the scope of test action $4^{4}$ influences the execution of $9^{5}, 10^{6}, 16^{7}$, and $17^{8}$, but it does not influence the execution of $13^{10}, 14^{11}$, $16^{12}$, and $17^{13}$. Let $\mathrm{M}(\mathrm{T})$ be a set of actions in a given trajectory $\mathrm{T}$. TC is a binary relation on $\mathrm{M}(\mathrm{T})$ defined bellow [Korel and Laski 90]. 
$\mathrm{X}^{\mathrm{p}} \mathrm{TC} \mathrm{Y}^{\mathrm{t}}, 1 \leq \mathrm{p}<\mathrm{t}$, iff

(1) $Y$ is in the scope of influence of $X$, and

(2) for all $k, p<k<t, T(k) \neq X$

where, the scope of influence is defined as follows:

(1) if $X$ then $B 1$ else $B 2$; Instruction $Y$ is in the scope of influence of $X$ iff $\mathrm{Y}$ is in $\mathrm{B} 1$ or $\mathrm{B} 2$.

(2) while $X$ do $B$; Instruction $Y$ is in the scope of influence of $X$ iff $Y$ is in $B$.

For example, in the program of Figure 2, instructions 5, 7, 9, 11, 13, 16, and 17 are in the scope of influence of test instruction 4, but instructions 18 and 19 are not. The TC Relation for the trajectory in Figure 10 is shown in Figure 13.

5. Compute the slicing set $\mathrm{S}_{\mathrm{C}}$ using the following definitions [Korel and Laski 90].

5.1 Let $X^{p}$ IR $Y^{t}$, iff $X=Y$ is the identity Relation IR on $M(\operatorname{Front}(T, q))$. The IR Relation for the trajectory in Figure 10 is obtained as shown in Figure 14.

$$
\begin{array}{ll}
\operatorname{TC}\left(4^{4}\right) & =\left\{9^{5}, 10^{6}, 16^{7}, 17^{8}\right\} \\
\operatorname{TC}\left(4^{9}\right) & =\left\{13^{10}, 14^{11}, 16^{12}, 17^{13}\right\} \\
\operatorname{TC}\left(4^{14}\right) & =\{\} \\
\operatorname{TC}\left(9^{5}\right) & =\left\{10^{6}\right\} \\
\operatorname{TC}\left(13^{10}\right) & =\left\{14^{11}\right\}
\end{array}
$$

Figure 13. The TC (test-control) relation for the trajectory depicted in Figure 10

$\begin{array}{ll}\operatorname{IR}\left(4^{4}\right) & =\left\{4^{9}, 4^{14}\right\} \\ \operatorname{IR}\left(4^{9}\right) & =\left\{4^{14}\right\} \\ \operatorname{IR}\left(4^{14}\right) & =\{\}\end{array}$

Figure 14. The IR (identity relation) relation for the trajectory depicted in Figure 10 
5.2 Let $\mathrm{C}=\left(\mathrm{x}, \mathrm{I}^{\mathrm{q}}, \mathrm{V}\right)$ be a slicing criterion and $\mathrm{T}$ be a trajectory on input $\mathrm{x}$. To find the slicing set $S_{C}$, we first find the set $A^{0}$ of all actions that have direct influence on $V$ at $q$ and on action $I^{q} \cdot A^{0}$ is defined as follows

$$
A^{0}=L D(q, V) \cup L T\left(I^{q}\right)
$$

where, $\mathrm{LD}(\mathrm{q}, \mathrm{V})$ is the set of last definitions of variables in $\mathrm{V}$ at the execution position $\mathrm{q}$, and $L T^{P}\left(I^{\mathfrak{p}}\right)$ is a set of test actions which have Test-Control influence on $I^{q}$.

$\mathrm{S}_{\mathrm{C}}$ can be determined iteratively as the limit of a sequence $\mathrm{S}^{0}, \mathrm{~S}^{1}, \ldots, \mathrm{S}^{\mathrm{n}}, 0 \leq \mathrm{n}<$ $\mathrm{q}$, which is defined as follows

$$
S^{0}=A^{0} \text { and } S^{i+1}=S^{i} \cup A^{i+1}
$$

where $A^{i+1}=\left\{X^{p} \in \mathbb{M}(T): 1 \leq p<q\right.$,

$$
\begin{aligned}
& \text { (1) } X^{p} \notin S^{i} \text {, and } \\
& \text { (2) there exists } \left.Y^{t} \in S^{i}, t<q, X^{p} Z Y^{t}\right\} \\
& \text { where } Z=D U \cup T C \cup I R \text {. }
\end{aligned}
$$

Finally, we can get the slice from the following definition.

$$
S_{c}=S^{k} \cup\left\{I^{q}\right\}
$$

where $S^{k}$ is the limit of the sequence $\left\{S^{i}\right\}$.

Example 1. Consider again trajectory $\mathrm{T}$ in Figure 10. Using the criterion

$\mathrm{C} 1=\left(\mathrm{x}, 19^{16},\{\right.$ CountNumber $\left.[1]\}\right), \mathrm{x}=($ MaxData, CountNumber $)=(2,(3,5))$, we have

$$
\begin{aligned}
& \operatorname{LD}(16, \text { CountNumber }[1]\})=\{\}, \operatorname{LT}\left(19^{16}\right)=\{\}, \\
& A^{0}=\{\}, \quad S^{0}=\{\}, \\
& S_{c 1}=S^{0} \cup\left\{19^{16}\right\}=\left\{19^{16}\right\} .
\end{aligned}
$$

And finally, the dynamic slice is shown in Figure 15. 


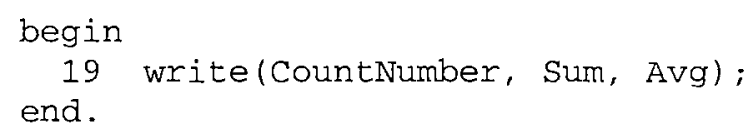

Figure 15. A dynamic program slice computed based on variable CountNumber[1] in line 19 of the program in Figure 2

Example 2. Consider again trajectory $\mathrm{T}$ in Figure 10. Using the criterion $\mathrm{C} 2=\left(\mathrm{x}, 19^{16},\{\right.$ CountNumber $\left.[5]\}\right), \mathrm{x}=($ MaxData, CountNumber $)=(2,(3,5))$, we have

$$
\begin{aligned}
& \operatorname{LD}(16,\{\text { CountNumber }[5]\})=\left\{14^{11}\right\}, \operatorname{LT}\left(19^{16}\right)=\{\}, \\
& A^{0}=\left\{14^{11}\right\}, \quad S^{0}=\left\{14^{11}\right\}, \\
& A^{1}=\left\{4^{9}, 13^{10}\right\}, \quad S^{1}=\left\{4^{9}, 13^{10}, 14^{11}\right\} \\
& A^{2}=\left\{1^{1}, 4^{4}, 17^{8}\right\}, \quad S^{2}=\left\{1^{1}, 4^{4}, 17^{8}, 4^{9}, 13^{10}, 14^{11}\right\} \\
& A^{3}=\left\{2^{2}\right\}, \quad S^{3}=\left\{1^{1}, 2^{2}, 4^{4}, 17^{8}, 4^{9}, 13^{10}, 14^{11}\right\} \\
& A^{4}=\{\}, \\
& S_{c 2}=S^{3} \cup\left\{19^{16}\right\}=\left\{1^{1}, 2^{2}, 4^{4}, 17^{8}, 4^{9}, 13^{10}, 14^{11}, 19^{16}\right\}
\end{aligned}
$$

And finally, the dynamic slice is shown in Figure 16.

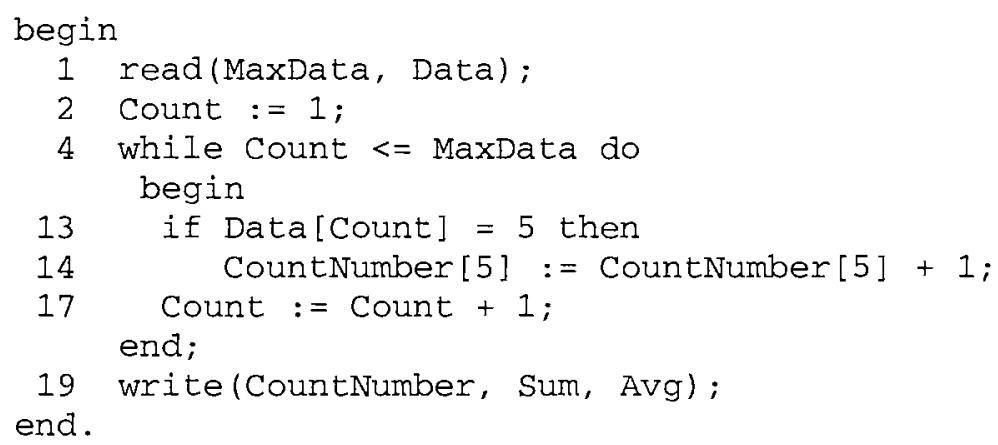

Figure 16. A dynamic program slice computed based on variable CountNumber [5] in line 19 of the program in Figure 2 
Example 3. Consider again trajectory $\mathrm{T}$ in Figure 10. Using the criterion

$$
\begin{aligned}
& \mathrm{C} 3=\left(\mathrm{x}, 19^{16},\{\text { Sum }\}\right), \mathrm{x}=(\text { MaxData }, \text { CountNumber })=(2,(3,5)) \text {, we have } \\
& \operatorname{LD}(16,\{\operatorname{Sum}\})=\left\{16^{12}\right\}, \operatorname{LT}\left(19^{16}\right)=\{\}, \\
& A^{0}=\left\{16^{12}\right\}, \quad S^{0}=\left\{16^{12}\right\}, \\
& A^{1}=\left\{1^{1}, 16^{7}, 17^{8}, 4^{9}\right\}, \quad S^{1}=\left\{1^{1}, 16^{7}, 17^{8}, 4^{9}, 16^{12}\right\}, \\
& A^{2}=\left\{2^{2}, 3^{3}, 4^{4}\right\}, \quad S^{2}=\left\{1^{1}, 2^{2}, 3^{3}, 4^{4}, 16^{7}, 17^{8}, 4^{9}, 16^{12}\right\}, \\
& \mathrm{A}^{3}=\{\}, \\
& S_{c 3}=S^{2} \cup\left\{19^{16}\right\}=\left\{1^{1}, 2^{2}, 3^{3}, 4^{4}, 16^{7}, 17^{8}, 4^{9}, 16^{12}, 19^{16}\right\} \text {. }
\end{aligned}
$$

And finally, the dynamic slice is shown in Figure 17.

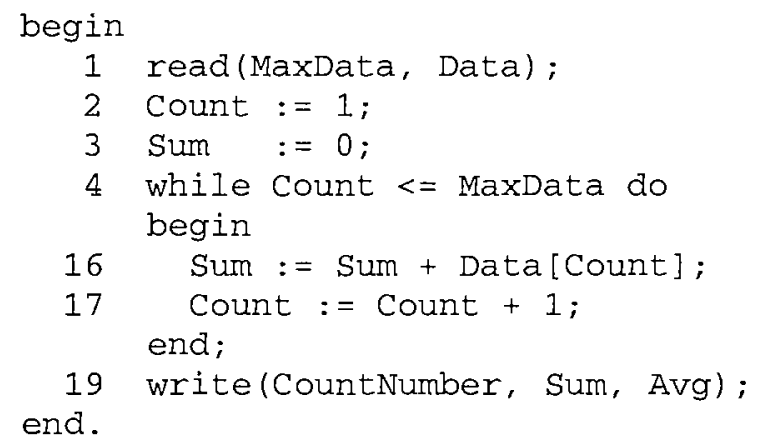

Figure 17. A dynamic program slice computed based on variable Sum in line 19 of the program in Figure 2

Example 4. Consider again trajectory $\mathrm{T}$ in Figure 10. Using the criterion

$$
\begin{aligned}
& \mathrm{C} 4=\left(\mathrm{x}, 19^{16},\{\text { Avg }\}\right), \mathrm{x}=(\text { MaxData }, \text { CountNumber })=(2,(3,5)) \text {, we have } \\
& \operatorname{LD}(16,\{\text { Avg }\})=\left\{18^{15}\right\}, \operatorname{LT}\left(19^{16}\right)=\{\}, \\
& A^{0}=\left\{18^{15}\right\}, \quad S^{0}=\left\{18^{15}\right\}, \\
& A^{1}=\left\{1^{1}, 16^{12}\right\}, \quad S^{1}=\left\{1^{1}, 16^{12}, 18^{15}\right\}, \\
& \mathrm{A}^{2}=\left\{16^{7}, 17^{8}, 4^{9}\right\}, \quad \mathrm{S}^{2}=\left\{1^{1}, 16^{7}, 17^{8}, 4^{9}, 16^{12}, 18^{15}\right\},
\end{aligned}
$$




$$
\begin{aligned}
& A^{3}=\left\{2^{2}, 3^{3}, 4^{4}\right\}, \quad S^{3}=\left\{1^{1}, 2^{2}, 3^{3}, 4^{4}, 16^{7}, 17^{8}, 4^{9}, 16^{12}, 18^{15}\right\} \\
& A^{4}=\{\}, \\
& S_{C 4}=S^{3} \cup\left\{19^{16}\right\}=\left\{1^{1}, 2^{2}, 3^{3}, 4^{4}, 16^{7}, 17^{8}, 4^{9}, 16^{12}, 18^{15}, 19^{16}\right\}
\end{aligned}
$$

And finally, the dynamic slice is shown in Figure 18.

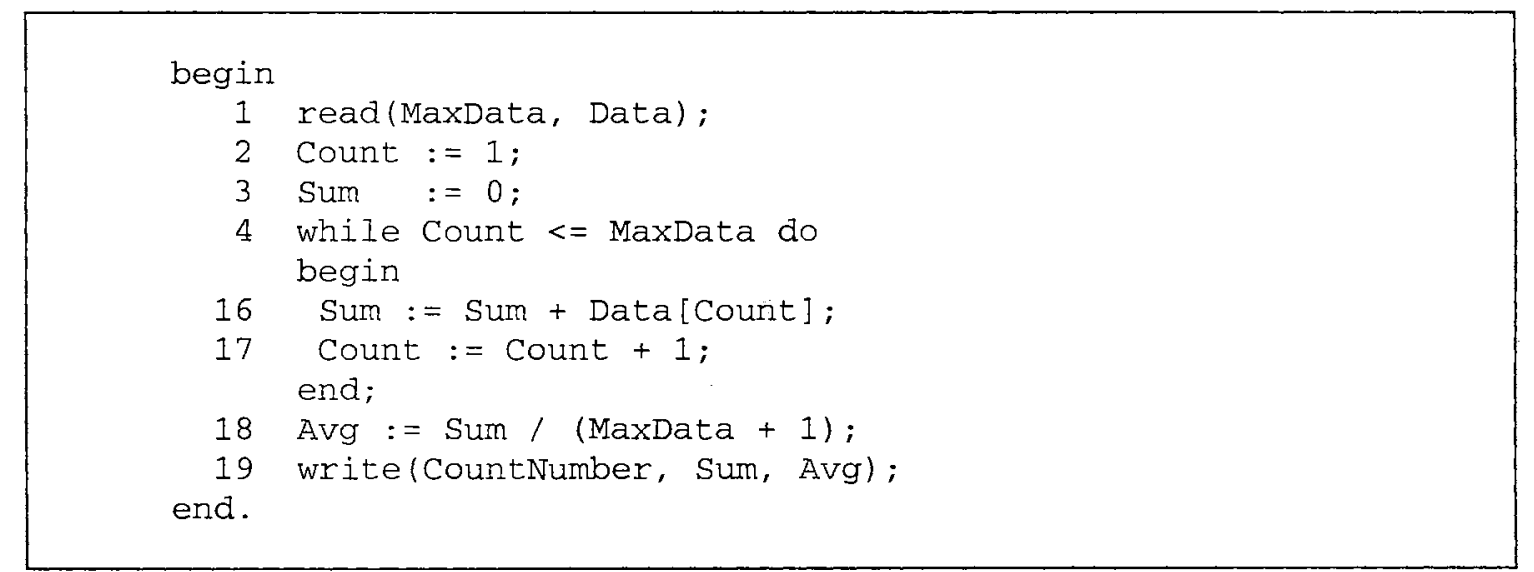

Figure 18. A dynamic program slice computed based on variable Avg in line 19 of the program in Figure 2

\subsection{Dicing Procedures}

Dicing [Lyle 84] [Nanja 90] [Nanja and Samadzadeh 90] is the process of identifying a set of statements likely to contain an error. A dice is determined using the following process.

1. Compute the slice $\left(S_{i}\right)$ for the incorrectly valued output variable(s), which is a subset of $\mathrm{KBI}$ (known to be incorrect).

2. Compute the slice $\left(S_{C}\right)$ for the correctly valued output variable(s), which is a subset of CSF (correct so far).

3. Compute $\left(S_{i}-S_{C}\right)$, which makes up the dice. 
Example 5. Observe that the dynamic program slice in Example 3 is a subset of CSF, while the dynamic program slice in Example 4 is a subset of KBI. Consequently, using the definition of dicing, a dice program can be shown in Figure 19.

\section{Avg := Sum / (MaxData + 1);}

Figure 19. The final program segment after slicing and dicing

Once the procedure is finished, line 18 will be shown as the incorrect line.

\subsection{Problems with Slices}

Although a number of significant advantages exist with the use of program slicing, program slicing does have disadvantages [Weiser 84]. These disadvantages can be summarized as follows. First, slices can be expensive to find. Second, a program may contain no significant slices other than itself. Third, total independence of slices may result in additional complexity in each slice that could be cleaned up if simple dependencies could be identified among slices. Finally, the selection of variables for slicing and dicing could pose significant problems. However, it can be asserted that whenever the program to be debugged is large, program slicing could effectively be used. 
CHAPTER III

\section{C++ DYNAMIC SLICING AND DICING PROCEDURES}

\subsection{Introduction}

A number of definitions and algorithms originally introduced by Korel and Laski [Korel and Laski 90] were modified, in order to compute slices in classes, objects, arrays, pointers, references, dynamic allocation operators, function overloading, copy constructors, default arguments, operator overloading, inheritance, virtual functions, polymorphism, templates, and exception handling of a $\mathrm{C}++$ program. Those modified definitions plus a number of new definitions and algorithms are introduced in this chapter.

\subsection{Definitions}

Based on Korel and Laski's work [Korel and Laski 90], let the flow graph of a program $\mathrm{P}$ be a directed graph $(\mathrm{N}, \mathrm{A}, \mathrm{S}, \mathrm{e})$ and $\mathrm{C}$ be a slicing criterion, where $\mathrm{N}$ is a set of nodes, $\mathrm{A}$ is a binary relation on $\mathrm{N}$ (a subset of $\mathrm{N} \times \mathrm{N}$ ) referred to as the set of arcs, $\mathrm{s} \in$ $\mathrm{N}$ is a unique entry node, and $e \in \mathrm{N}$ is a unique exit node.

Each node in $\mathrm{N}$ consists of one statement, including a single instruction, a control instruction, and a function instruction. A single instruction can be, for example, an 
assignment statement or an input or output statement. A control instruction can be such statements as an if-then-else statement or a while statement, which are also called test instructions. A function instruction can be either a called or a calling function instruction.

$\operatorname{An} \operatorname{arc}(n, m) \in A$ corresponds to a possible transfer of control from instruction $n$ to instruction $\mathrm{m}$.

A path from the entry node $s$ to some node $k, k \in N$, is called a sequence $<n_{1}$, $n_{2}, \ldots, n_{q}>$ of instructions, such that $n_{1}=s, n_{q}=k$, and $\left(n_{i}, n_{i+1}\right) \in A$, for all $n_{i}, 1 \leq i$ $<$ q. If there are input data that cause a path to be traversed during program execution, the path is feasible. A feasible path that has actually been executed for some input is called a trajectory.

The program in Figure 20 is designed to compute the factorial of a given number Num. For example, if Num $=3$ the program yields the result of 6 . Figure 21 shows a trajectory of the program in Figure 20 on input data Num $=3$.

\section{Definition 1}

Let $X$ be an instruction in a program and $X \in \mathbb{I N}^{+}$(the set of non-negative integers). Let $P$ be the set of instruction numbers in a tested $C++$ program, then $P=\{1$, $2, \ldots, n\}$ represents a program of length $n$, where $n$ is the size of the program. For example, the $\mathrm{C}++$ program in Figure 20 is the program $P=\{1,2, \ldots, 25\}$, where \#include <iostream $>$ is instruction $X=1$, int Fac(int $N)$ is instruction $X=3$, etc.

$$
P=\{x \mid \text { for all } x \text { with } 1 \leq x \leq n\}
$$




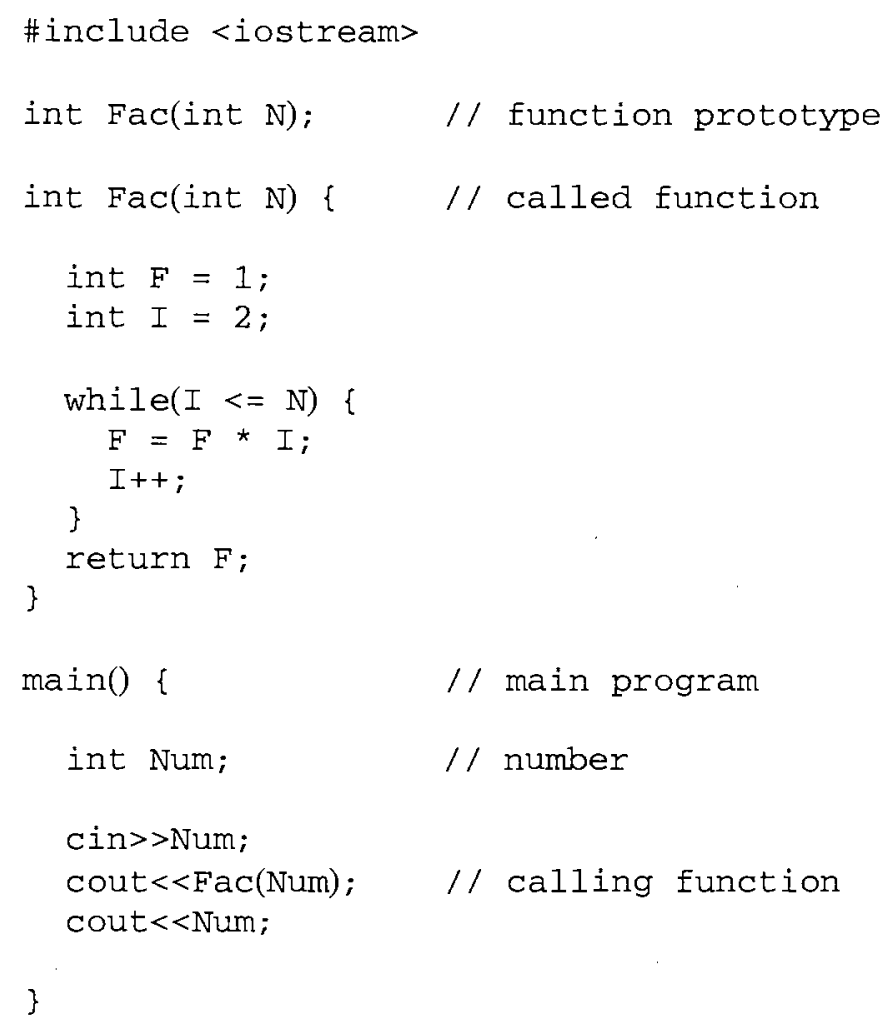

Figure 20. A program for computing the factorial of a number

where $\mathrm{n}=$ length of the program.

\section{Definition 2}

Let $F_{\text {name }}$ be a function, i.e., a set of instruction $X^{\prime} s$ in the scope of influence of the function name, where all blank lines are ignored. For example, in Figure $20, F_{F a c}=$ $\{5,7,8,10,11,12,13,14,15\}$ and $F_{\text {main }}=\{17,19,21,22,23,25\} . F_{\text {name }} \subseteq P$, and $F_{\text {name }}=F_{\text {main }}$ if the program has one function.

$$
F_{\text {name }}=\{x \mid \text { for all } x \text { with } i \leq x \leq k\}
$$

where (1) $i$ is the starting line number of function name, $i \in P$

(2) $\mathrm{k}$ is the ending line number of function name, $\mathrm{k} \in \mathrm{P}$ 


\section{Definition 3}

Let $\mathrm{T}$ be a trajectory, i.e., a feasible path that has actually been executed for some input [Korel and Laski 90]. A trajectory of length $\mathrm{m}$ is denoted by a list $\mathrm{T}=<\mathrm{X}_{1}, \mathrm{X}_{2}, \ldots$, $\mathrm{X}_{\mathrm{m}}>$, where $\mathrm{X}$ is an instruction of a tested $\mathrm{C}++$ program. For example, in Figure $21,<17$, $19,21,5,7,8,10,11,12,13,10,11,12,13,14,22,23,25>$ is the resulting trajectory when the program in Figure 20 is executed on input data Num $=3$.

$$
\begin{aligned}
& \mathrm{T}=\langle\mathrm{X}| \text { for all } \mathrm{X}, \text { where } \mathrm{X} \text { 's are in a feasible path executed for some input } \\
& \text { and } \mathrm{X} \in \mathrm{P}>
\end{aligned}
$$

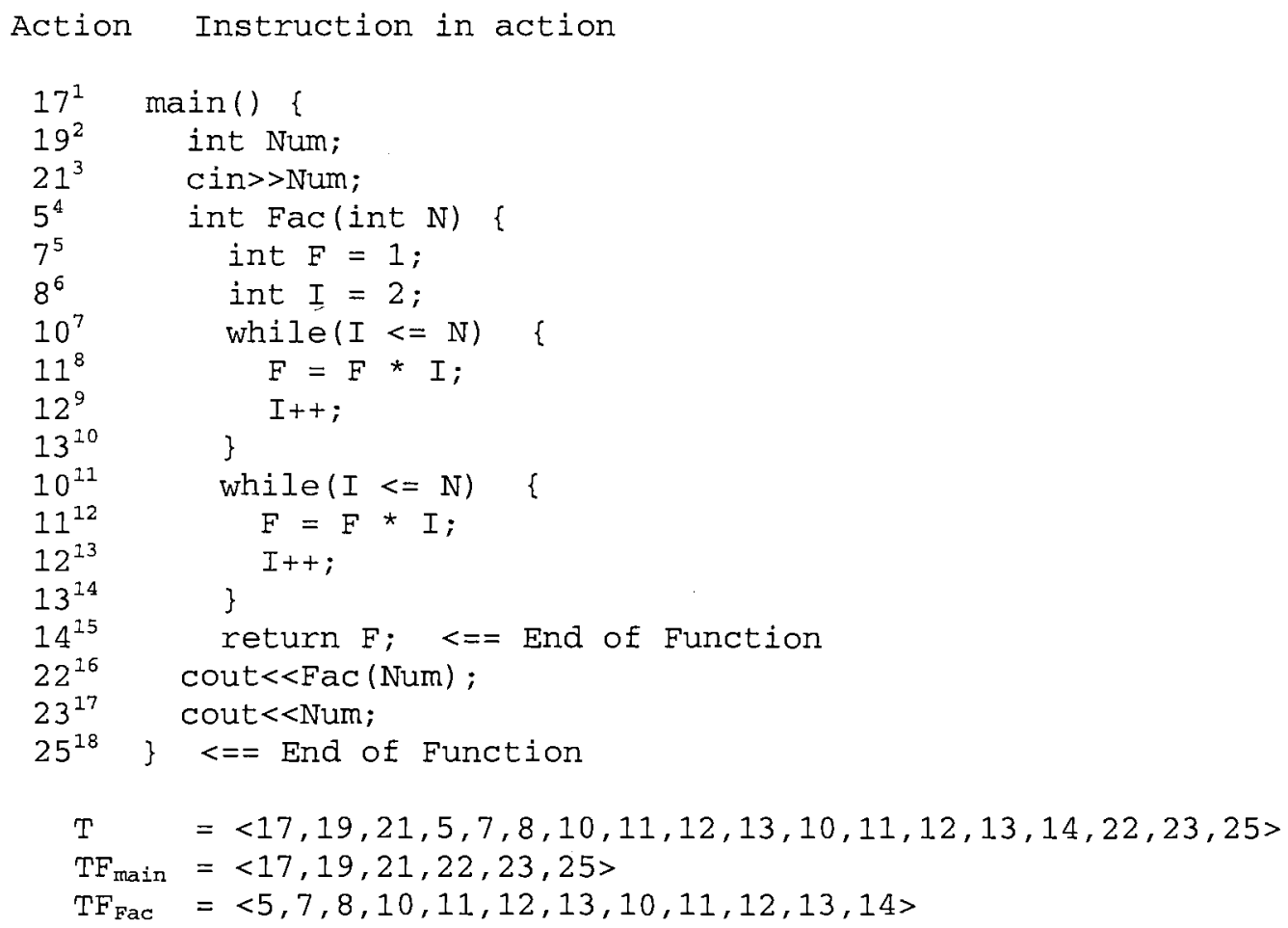

Figure 21. A trajectory of the program in Figure 20 on input data Num $=3$

\section{Definition 4}

Let $\mathrm{TF}_{\text {name }}$ be a function trajectory, i.e., a feasible path of a function name that 
has actually been executed for some input. $\mathrm{TF}_{\text {name }}$ is a sublist of $\mathrm{T}$. If a trajectory of length $\mathrm{m}$ is denoted by $\mathrm{T}=\left\langle\mathrm{X}_{1}, \mathrm{X}_{2}, \ldots, \mathrm{X}_{\mathrm{m}}\right\rangle$, then the function trajectory name is denoted by $\mathrm{TF}_{\text {name }}=\left\langle\mathrm{X}_{\mathrm{i}}, \mathrm{X}_{\mathrm{i}+1}, \ldots, \mathrm{X}_{\mathrm{k}}\right\rangle$, where $\mathrm{X}_{1}, \mathrm{X}_{\mathrm{i}+1}, \ldots, \mathrm{X}_{\mathrm{k}}$ are a list of the instruction $\mathrm{X}$ 's which are in the scope of a given function $F_{\text {name, }}$, where $i$ denotes the position of entry node and $k$ denotes the position of ending node of the function name, $(1 \leq i<k$, and $i<$ $\mathrm{k} \leq \mathrm{m}$ ). For example, in Figure $21,<17,19,21,22,23,25>$ is the trajectory of $\mathrm{TF}_{\text {main }}$, and $\left\langle 5,7,8,10,11,12,13,10,11,12,13,14>\right.$ is the trajectory of $\mathrm{TF}_{\mathrm{Fac}}$, when the program in Figure 20 is executed on input data Num $=3$.

$\begin{aligned} \mathrm{TF}_{\text {name }}= & <\mathrm{X} \mid \text { for all } \mathrm{X}, \text { where } \mathrm{X} \text { 's are in a feasible path executed for some input, } \\ & \mathrm{X} \in \mathrm{F}_{\text {name }} \text {, and } \mathrm{X} \in \mathrm{T}>\end{aligned}$

Definition 5

Let action be pair $(\mathrm{X}, \mathrm{p})$, i.e., instruction $\mathrm{X}$ at position $\mathrm{p}$, which will be replaced by $\mathrm{X}^{\mathrm{p}}$ for brevity and ease of understanding [Korel and Laski 90 ]. For example, $11^{8}$ and $11^{12}$ in trajectory $\mathrm{T}$ in Figure 21 are actions that involve the same instruction 11 . An action $\mathrm{X}^{\mathrm{p}}$ is a test action if $\mathrm{X}$ is a test instruction such as while or for. For example, $10^{7}$ and $10^{11}$ in trajectory $\mathrm{T}$ in Figure 21 are test actions.

\section{Definition 6}

Let $M(T)$ be a set of actions in a given trajectory $T$, where $M(T)=\left\{X^{p}\right.$ : instruction $X$ at position $p$ in trajectory $T\}$ [Korel and Laski 90]. For example, in Figure 21, $\left\{17^{1}\right.$, $\left.19^{2}, 21^{3}, 5^{4}, 7^{5}, 8^{6}, 10^{7}, 11^{8}, 12^{9}, 13^{10}, 10^{11}, 11^{12}, 12^{13}, 13^{14}, 14^{15}, 22^{16}, 23^{17}, 25^{18}\right\}$ is a set of actions $\mathrm{M}(\mathrm{T})$. 


\section{Definition 7}

Let $\mathrm{M}\left(\mathrm{TF}_{\text {name }}\right)$ be a set of actions in a given function of a given trajectory $\mathrm{TF}_{\text {name, }}$ where $M\left(T F_{\text {name }}\right)=\left\{X^{p}\right.$ : instruction $X$ at position $p$ in trajectory $\left.T F_{\text {name }}\right\} \cdot M\left(T F_{\text {name }}\right)$ is a subset of $\mathrm{M}(\mathrm{T})$. For example, in Figure $21,\left\{17^{1}, 19^{2}, 21^{3}, 22^{16}, 23^{17}, 25^{18}\right\}$ is a set of actions $\mathrm{M}\left(\mathrm{TF}_{\text {main }}\right)$, and $\left\{5^{4}, 7^{5}, 8^{6}, 10^{7}, 11^{8}, 12^{9}, 13^{10}, 10^{11}, 11^{12}, 12^{13}, 13^{14}, 14^{15}\right\}$ is a set of actions $\mathrm{M}\left(\mathrm{TF}_{\mathrm{Fac}}\right)$.

\section{Definition 8}

Let $\mathrm{C}$ be a slicing criterion, which is the specification for a particular behavior of interest (see Subsection 2.5.2 for more detail). A slicing criterion can be expressed as the values of some set of variables at some set of statements [Weiser 81]. If we let $\mathrm{T}$ be the trajectory of program $\mathrm{P}$ on input $\mathrm{x}$, a slicing criterion of program $\mathrm{P}$ executed on $\mathrm{x}$ can be defined as a triple $\mathrm{C}=\left(\mathrm{x}, \mathrm{I}^{\mathrm{q}}, \mathrm{V}\right)$, where $\mathrm{I}^{\mathrm{q}}$ is an action in $\mathrm{T}$ and $\mathrm{V}$ is a subset of variables in P [Korel and Laski 90].

\section{Definition 9}

Let $D\left(X^{p}\right)$ be the set of variables that are defined in action $X^{p}$, where $X^{p} \in M(T)$. For example, in the trajectory of Figure 21,

$$
21^{3} \text { Cin>>Num; }
$$

Num is a set of variables that are defined in $21^{3}, D\left(21^{3}\right)=\{\mathrm{Num}\}$.

Let $\mathrm{DF}_{\text {name }}\left(\mathrm{X}^{\mathrm{p}}\right)$ be the set of variables that are defined in action $\mathrm{X}^{\mathrm{p}}$, where $\mathrm{X}^{\mathrm{p}} \in$ $\mathrm{M}\left(\mathrm{TF}_{\text {name }}\right)$. In Figure 21 , since $21^{3} \in \mathrm{M}\left(\mathrm{TF}_{\text {main }}\right)$ and Num is a set of variables that are defined in function main, $\mathrm{DF}_{\text {main }}\left(21^{3}\right)=\{\mathrm{Num}\}$ 
Definition 10

Let $U\left(X^{p}\right)$ be the set of variables that are used in action $X^{\mathrm{p}}$, where $\mathrm{X}^{\mathrm{p}} \in \mathrm{M}(\mathrm{T})$. For example in the trajectory of Figure 21,

$$
10^{11} \text { while }(\mathrm{I}<=\mathrm{N}) \quad\{
$$

$I$ and $N$ are the set of variables that are used in $10^{11}, U\left(10^{11}\right)=\{I, N\}$.

Let $\mathrm{UF}_{\text {name }}\left(\mathrm{X}^{\mathrm{p}}\right)$ be the set of variables that are used in action $\mathrm{X}^{\mathrm{p}}$, where $\mathrm{X}^{\mathrm{p}} \in$ $\mathrm{M}\left(\mathrm{TF}_{\text {name }}\right)$. From last example, since $10^{11} \in \mathrm{M}\left(\mathrm{TF}_{\mathrm{Fac}}\right)$ and $\mathrm{I}$ and $\mathrm{N}$ are the set of variables that are used in function Fac, $U_{F a c}\left(10^{11}\right)=\{I, N\}$.

\section{Definition 11}

Let $\mathrm{LF}_{\text {name }}\left(\mathrm{X}^{\mathrm{P}}\right)$ be a set of variables and $\mathrm{C}++$ preprocessors that are declared as a local declaration in function name. For example, in the trajectory of Figure 21, $\operatorname{LF}_{\mathrm{Fac}}\left(7^{5}\right)=\{F\}, L F_{\mathrm{Fac}}\left(8^{6}\right)=\{I\}$, and $L F_{\text {main }}\left(19^{2}\right)=\{$ Num $\}$. There are no local $\mathrm{C}++$ preprocessors in this example.

\section{Definition 12}

Let $\mathrm{DU}$ be a Definition-Use Relation, a relation in which one action assigns a value to an item of data and the other action uses that value [Korel and Laski 90]. For example, in the trajectory of Figure $21,11^{12}$ assigns a value to variable $\mathrm{F}$ and $14^{15}$ use that value. Instead of using $\mathrm{M}(\mathrm{T})$ as Korel and Laski did, $\mathrm{M}\left(\mathrm{TF}_{\text {name }}\right)$ was used in this work in order to compute a slice from functions or classes. 


\begin{tabular}{|c|c|c|c|}
\hline $\mathrm{M}\left(\mathrm{TF}_{\mathrm{FaC}}\right)$ & $\mathrm{DF}_{\mathrm{Fac}}\left(\mathrm{X}^{\mathrm{p}}\right)$ & $\mathrm{UF}_{\text {Fad }}\left(\mathrm{X}^{\mathrm{D}}\right)$ & $L F_{\mathrm{FaC}}\left(\mathrm{X}^{\mathrm{p}}\right)$ \\
\hline $\begin{array}{c}5^{4} \\
7^{5} \\
8^{6} \\
10^{7} \\
11^{8} \\
12^{9} \\
13^{10} \\
10^{11} \\
11^{12} \\
12^{13} \\
13^{14} \\
14^{15}\end{array}$ & $\begin{array}{l}\text { F } \\
I \\
F \\
I\end{array}$ & $\begin{array}{l} \\
I, \quad N \\
F, I \\
I \\
I, \quad N \\
F, I \\
I \\
F\end{array}$ & $\begin{array}{l}\mathrm{N} \\
\mathrm{F} \\
\mathrm{I}\end{array}$ \\
\hline
\end{tabular}

Figure 22. The sets $M\left(\mathrm{TF}_{\mathrm{Fac}}\right), \mathrm{DF}_{\mathrm{Fac}}\left(\mathrm{X}^{\mathrm{p}}\right), \mathrm{UF}_{\mathrm{Fac}}\left(\mathrm{X}^{\mathrm{p}}\right)$, and $\mathrm{LF} \mathrm{Fac}_{\mathrm{F}}\left(\mathrm{X}^{\mathrm{p}}\right)$ for the trajectory in Figure 21

\begin{tabular}{|l|l|l|l|}
\hline $\mathrm{M}\left(\mathrm{TF}_{\text {main }}\right)$ & $\mathrm{DF}_{\text {main }}\left(\mathrm{X}^{\mathrm{D}}\right)$ & $\mathrm{UF}_{\text {main }}\left(\mathrm{X}^{\mathrm{D}}\right)$ & $\mathrm{LF}_{\text {main }}\left(\mathrm{X}^{\mathrm{D}}\right)$ \\
\hline $17^{1}$ & & & \\
$19^{2}$ & & & Num \\
$21^{3}$ & Num & Num & \\
$22^{16}$ & & Num & \\
$23^{17}$ & & & \\
$25^{18}$ & & & \\
\hline
\end{tabular}

Figure 23. The sets $\mathrm{M}\left(\mathrm{TF}_{\text {main }}\right), \mathrm{DF}_{\text {main }}\left(\mathrm{X}^{\mathrm{p}}\right), \mathrm{UF}_{\text {main }}\left(\mathrm{X}^{\mathrm{p}}\right)$, and $\mathrm{LF} \mathrm{F}_{\text {main }}\left(\mathrm{X}^{\mathrm{p}}\right)$ for the trajectory in Figure 21

Let $M\left(T F_{\text {name }}\right)$ be a set of actions in a given trajectory $T F_{\text {name. }} D U F_{\text {name, }}$ a Definition-Use-Function $_{\text {name }}$ Relation, is a binary relation on $\mathrm{M}\left(\mathrm{TF}_{\text {name }}\right)$ defined as follows: 
Let $\mathrm{TF}_{\text {name }}=\left\langle\mathrm{X}_{\mathrm{i}}, \mathrm{X}_{\mathrm{i}+1}, \ldots, \mathrm{X}_{\mathrm{t}}, \ldots, \mathrm{X}_{\mathrm{k}}>\right.$,

$\mathrm{X}^{\mathrm{p}} \mathrm{DUF}_{\text {name }} \mathrm{Y}^{\mathrm{t}}, \mathrm{i} \leq \mathrm{p}<\mathrm{t}$, iff there exists a variable $\mathrm{v}$

such that (1) $v \in U_{\text {name }}\left(Y^{t}\right)$, and

(2) $\mathrm{X}^{\mathrm{p}}$ is the last definition of $\mathrm{v}$ at $\mathrm{t}$

where, the last definition $\mathrm{X}^{\mathrm{p}}$ of variable $\mathrm{v}$ at $\mathrm{t}$ is the action which last assigned a value to $\mathrm{V}$ when $t$ was reached on trajectory $\mathrm{TF}_{\text {name. }}$.

For example, in the trajectory of Figure $21,21^{3}$ is the last definition of variable Num at the execution positions 4 through 18 . The $\mathrm{DUF}_{\text {name }}$ Relation for the trajectory in Figure 21 is shown in Figures 24 and 25.

\section{Definition 13}

Let LDR be a Local-Declaration Relation, a relation in which one action declares a variable and the other action defines or uses that variable. For example, in the trajectory of Figure $21,7^{5}$ declares variable $\mathrm{F}$ and $11^{8}, 11^{12}$ define and $11^{8}, 11^{12}, 14^{15}$ use that variable.

Let $\mathrm{M}\left(\mathrm{TF}_{\text {name }}\right)$ be a set of actions in a given trajectory $T F_{\text {name. }} L D R F_{\text {name, }}$ a LocalDeclaration $_{\text {name }}$ Relation, is a binary relation on $\mathrm{M}\left(\mathrm{TF}_{\text {name }}\right)$ defined as follows:

Let $\mathrm{TF}_{\text {name }}=\left\langle\mathrm{X}_{\mathrm{i}}, \mathrm{X}_{\mathrm{i}+1}, \ldots, \mathrm{X}_{\mathrm{t}}, \ldots, \mathrm{X}_{\mathrm{k}}>\right.$, $\mathrm{X}^{\mathrm{p}} \mathrm{LDRF}$ name $\mathrm{Y}^{\mathrm{t}}, \mathrm{i} \leq \mathrm{p}<\mathrm{t}$, iff there exists a variable $\mathrm{v}$ such that (1) $v \in U_{\text {name }}\left(Y^{t}\right) \cup D F_{\text {name }}\left(Y^{t}\right)$, and

(2) $\mathrm{X}^{\mathrm{P}}$ is the action where variable $\mathrm{V}$ was declared in trajectory $\mathrm{TF}_{\text {name. }}$.

The LDRF name Relation for the trajectory in Figure 21 is shown in Figures 26 and 27. 


$$
\begin{array}{ll}
\operatorname{DUF}_{\mathrm{FaC}}\left(11^{8}\right) & =\left\{11^{12}\right\} \\
\operatorname{DUF}_{\mathrm{FaC}}\left(12^{9}\right) & =\left\{10^{11}, 11^{12}, 12^{13}\right\} \\
\operatorname{DUF}_{\mathrm{FaC}}\left(11^{12}\right) & =\left\{14^{15}\right\} \\
\operatorname{DUF}_{\mathrm{FaC}}\left(12^{13}\right) & =\{\}
\end{array}
$$

Figure 24. The DUF ${ }_{\text {Fac }}$ relation for the trajectory depicted in Figure 21

$$
\operatorname{DUF}_{\text {main }}\left(21^{3}\right) \quad=\left\{22^{15}, 23^{17}\right\}
$$

Figure 25. The $\mathrm{DUF}_{\text {main }}$ relation for the trajectory depicted in Figure 21

$$
\begin{array}{ll}
\operatorname{LDRF}_{\text {Fac }}\left(5^{4}\right) & =\left\{10^{7}, 10^{11}\right\} \\
\operatorname{LDRF}_{\text {Fac }}\left(7^{5}\right) & =\left\{11^{8}, 11^{12}, 14^{15}\right\} \\
\operatorname{LDRF}_{\text {Fac }}\left(8^{6}\right) & =\left\{10^{7}, 11^{8}, 12^{9}, 10^{11}, 11^{12}, 12^{13}\right\}
\end{array}
$$

Figure 26. The LDRF $F_{\text {ac }}$ relation for the trajectory depicted in Figure 21

$$
\operatorname{LDRF}_{\text {main }}\left(19^{2}\right) \quad=\left\{21^{3}, 22^{16}, 23^{17}\right\}
$$

Figure 27. The $L D R F_{\text {main }}$ relation for the trajectory depicted in Figure 21

\section{Definition 14}

Let TC be a Test-Control Relation, capturing the effect between test actions and actions that have been chosen to execute by these test actions [Korel and Laski 90]. For example in the trajectory of Figure 21 , the scope of test action $10^{7}$ influences the execution of $11^{8}, 12^{9}$, and $13^{10}$, but it does not influence the execution of $10^{11}, 11^{12}$, and $12^{13}$. Instead of using $M(T)$ as Korel and Laski did, $M\left(T F_{n a m e}\right)$ was used in this work in order to compute a slice from functions or classes. Let $\mathrm{M}\left(\mathrm{TF}_{\text {name }}\right)$ be a set of actions in a 
given trajectory $\mathrm{TF}_{\text {name. }} \mathrm{TCF}$ name, a Test-Control-Function ${ }_{\text {name }}$ Relation, is a binary relation on $\mathrm{M}\left(\mathrm{TF}_{\text {name }}\right)$ defined as follows:

$$
\begin{aligned}
& \text { Let } \mathrm{TF}_{\text {name }}=<\mathrm{X}_{i}, \mathrm{X}_{\mathrm{i}+1}, \ldots, \mathrm{X}_{\mathrm{t}}, \ldots, \mathrm{X}_{\mathrm{k}}>\text {, } \\
& \mathrm{X}^{\mathrm{p}} \mathrm{TCF}_{\text {name }} \mathrm{Y}^{\mathrm{t}}, i \leq \mathrm{p}<\mathrm{t} \text {, iff }
\end{aligned}
$$

(1) $\mathrm{Y}$ is in the scope of influence of $\mathrm{X}$, and

(2) for all $k, p<k<t, T(k) \neq X$

where, the scope of influence is defined as follows.

(1) if $X$ then $B 1$ else $B 2$; Instruction $Y$ is in the scope of influence of $X$ iff $Y$ is in $\mathrm{B} 1$ or $\mathrm{B} 2$.

(2) while $X$ do $B$; Instruction $Y$ is in the scope of influence of $X$ iff $Y$ is in $B$.

(3) do $B$ while $X$; Instruction $Y$ is in the scope of influence of $X$ iff $Y$ is in $B$.

(4) case $X \mathrm{do} B$; Instruction $Y$ is in the scope of influence of $X$ iff $Y$ is in $B$.

(5) for $X$ do $B$; Instruction $Y$ is in the scope of influence of $X$ iff $Y$ is in $B$.

(6) function $X$ do $B$; Instruction $Y$ is in the scope of influence of $X$ iff $Y$ is in B.

For example, in the trajectory of Figure 21, instructions 11, 12, and 13 are in the scope of influence of test instruction 10, but instructions $17,19,21,5,7,8,14,22,23$, and 25 are not. The $\mathrm{TCF}_{\text {name }}$ Test-Control-Function ${ }_{\text {name }}$ Relation for the trajectory in Figure 21 is shown in Figure 28.

$$
\begin{array}{ll}
\operatorname{TCF}_{\text {Fac }}\left(10^{7}\right) & =\left\{11^{8}, 12^{9}, 13^{10}\right\} \\
\operatorname{TCF}_{\text {Fac }}\left(10^{11}\right) & =\left\{11^{12}, 12^{13}, 13^{14}\right\}
\end{array}
$$

Figure 28. The $\mathrm{TCF}_{\mathrm{Fac}}$ relation for the trajectory depicted in Figure 21

Definition 15

Let $I R F_{\text {name }}$ be an Identity Relation in Function name, $_{\text {, then }} \mathrm{X}^{\mathrm{P}} \operatorname{IRF}_{\text {name }} \mathrm{Y}^{\mathrm{t}}$, iff $\mathrm{X}=$ $\mathrm{Y}$ is the identity relation $I R F_{\text {name }}$ on $\mathrm{M}\left(\mathrm{Front}\left(\mathrm{TF}_{\text {name }}, \mathrm{q}\right)\right)$, where $\operatorname{Front}\left(\mathrm{TF} \mathrm{F}_{\text {name }}, \mathrm{q}\right)$ is 
a sublist of $\mathrm{TF}_{\text {name }}$ consisting of the first $\mathrm{q}$ elements of $\mathrm{TF}_{\text {name, }}$, where $T \mathrm{TF}_{\text {name }}=\left\langle\mathrm{X}_{i}\right.$, $\mathrm{X}_{\mathrm{i}+1}, \ldots, \mathrm{X}_{t}, \ldots, \mathrm{X}_{\mathrm{q}}, \ldots, \mathrm{X}_{\mathrm{k}}>$ denotes a function trajectory, $\mathrm{q}$ is a position in $\mathrm{TF}_{\text {name }}, 1 \leq i$ $<t$, and $t<q \leq k$. The $I R F_{\text {name }}$ Relation for the trajectory in Figure 21 is obtained as shown in Figure 29.

$$
\begin{array}{ll}
\operatorname{IRF}_{\text {Fac }}\left(10^{17}\right) & =\left\{10^{11}\right\} \\
\operatorname{IRF}_{\text {Fac }}\left(10^{11}\right) & =\left\{10^{17}\right\}
\end{array}
$$

Figure 29. The IRF $F_{\mathrm{Fac}}$ relation for the trajectory depicted in Figure 21

\section{Definition 16}

Figure 30 presents a part of the trajectory of FuncA(int $i$ ) and FuncB(int $j$ ), where called FuncA(int $i)$ is called by calling FuncA(5) at $x^{n+1}$, and called FuncB(int $j$ ) is called by calling $\operatorname{FuncB}(2)$ at $x^{1+1}$. From Figure 30, we find that $T=$ $<\ldots, x^{i-2}, x^{i-1}, x^{i}, x^{i+1}, x^{i+2}, \ldots, x^{j}, x^{j+1}, \ldots, x^{k}, x^{k+1}, \ldots, x^{1}, x^{1+1}, \ldots, x^{m}, \ldots, x^{n}, x^{n+1}$, $\mathrm{X}^{\mathrm{n}+2}, \ldots>$, where $i<j<k, 1<m<n$ and $\mathrm{X}$ is any statement in a program $\mathrm{P}, T F_{\mathrm{FuncA}}=<\mathrm{X}^{i}$,

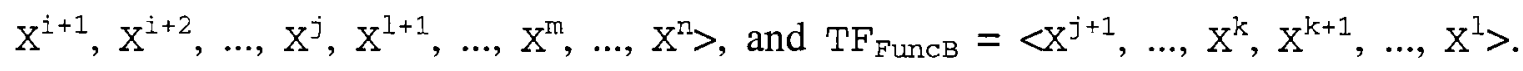
Functions $F u n C A(i n t ~ i)$ at $x^{i}$ and $F u n c B(i n t ~ j)$ at $x^{j+1}$ are called a called function instruction. An action $\mathrm{X}^{\mathrm{p}}$ is a called action if $\mathrm{x}$ is a called function instruction. Func $A(5)$ at $X^{n+1}$ and $\operatorname{FunCB}(2)$ at $x^{1+1}$ are called calling function instructions. An action $\mathrm{X}^{\mathrm{p}}$ is a calling action if $\mathrm{X}$ is a calling function instruction.

Called-to-Calling occurs when a slice is computed from a called action first and then from a calling action. For example, in Figure 31, suppose one needs to find a slice 


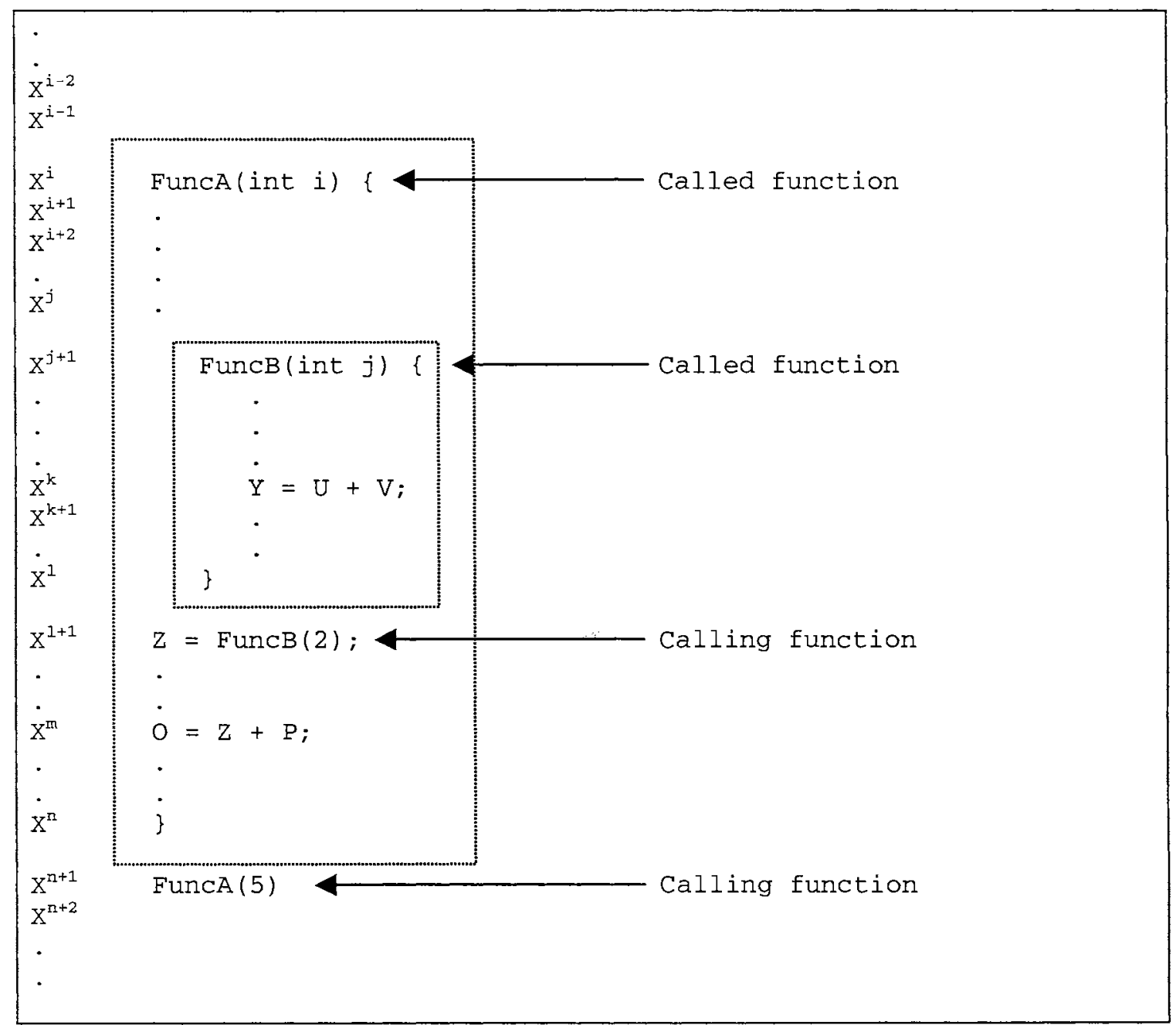

Figure 30. A trajectory of functions $\mathrm{A}$ and $\mathrm{B}$ where function $\mathrm{A}$ calls function $\mathrm{B}$

of variable $U$ at $X^{k}$. The process starts from $X^{k}$ (which is in the scope of influence of called function $F u n c B(i n t ~ j)$, which is called by calling function $\operatorname{FuncB}(2)$ at $X^{I+1}$ ), and then $\mathrm{X}^{j+1}, \mathrm{X}^{1+1}$, respectively. We find that called action $\mathrm{X}^{\mathrm{j}+1}$ comes before calling action $\mathrm{x}^{1+1}$

Calling-to-Called occurs when a slice is computed from a calling action first and then from a called action. For example, in Figure 32, suppose that one needs to find a slice of variable $Z$ at $X^{m}$. The process starts from $X^{m}$, and then $X^{1+1}$ (since $Z$ is last 


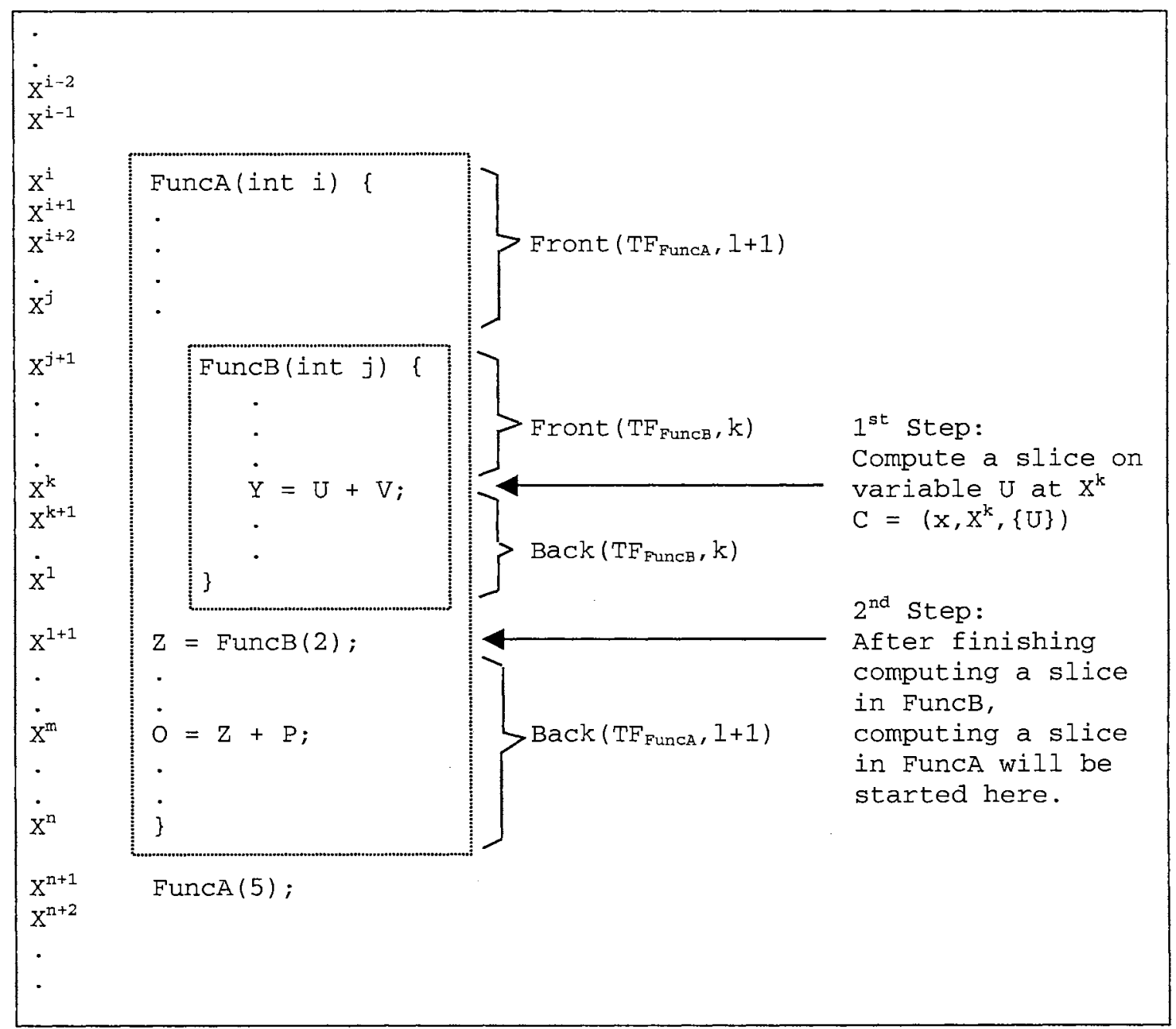

Figure 31. Illustrate Called-to-Calling

defined at $X^{1+1}$ and used at $X^{m}$ ) and then $X^{j+1}$ (since called FuncB(int $j$ ) is called by calling FuncB(2)), respectively. We find that calling action $\mathrm{X}^{1+1}$ comes before called action $\mathrm{X}^{\mathrm{j}+1}$.

Modified from Korel and Laski's approach [Korel and Laski 90], let $\mathrm{TF}_{\text {name }}=$ $<x_{i}, X_{i+1}, X_{i+2}, \ldots, X_{k}>$ be a trajectory of function name, and $q$ be a position in $\mathrm{TF}_{\text {name }}, i \leq \mathrm{q} \leq \mathrm{k}$. Then $\operatorname{Front}\left(\mathrm{TF}_{\text {name }}, \mathrm{q}\right)$ is a sublist $\left\langle\mathrm{X}_{i}, \mathrm{X}_{i+1}, \ldots, \mathrm{X}_{\mathrm{q}}\right\rangle$ and $\operatorname{Back}\left(T F_{\text {name }}, \mathrm{q}\right)$ is a sublist $<\mathrm{X}_{\mathrm{q}+1}, \mathrm{X}_{\mathrm{m}+2}, \ldots, \mathrm{X}_{\mathrm{k}}>$ as shown in Figures 31 and 32. All 


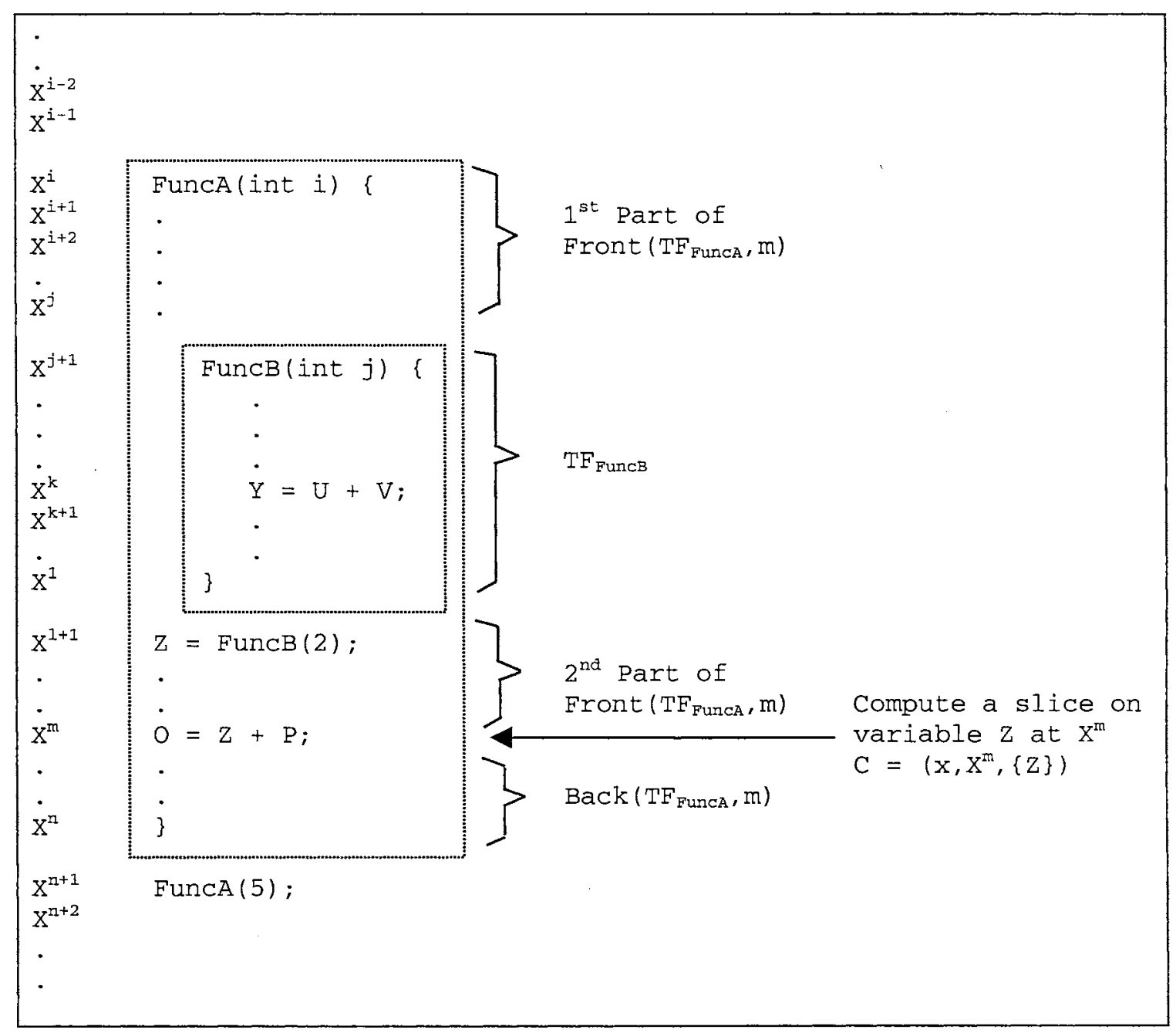

Figure 32. Illustrate Calling-to-Called

$\operatorname{Back}\left(\mathrm{TF}_{\text {name }}, \mathrm{q}\right)$ 's can be ignored in computing a slice. Just Front $\left(\mathrm{TF}_{\text {name }}, \mathrm{q}\right)$ must be concentrated on.

Let $A$ and $B$ be two functions, where function $A$ calls function $B$. Therefore, a slice can be computed in two different ways as follow.

1) Called-to-Calling

Total slice $_{\mathrm{AB}}=$ Slice $_{\mathrm{B}} \cup$ Slice $_{\mathrm{A}}$ where 
(1) Slice ${ }_{B}$ is a slice computed based on $\operatorname{Front}\left(\mathrm{TF}_{B}, \mathrm{k}\right)$ and slicing criterion $\mathrm{C}=\left(\mathrm{x}, \mathrm{X}^{\mathrm{k}}, \mathrm{V}\right)$

(2) Slice $_{A}$ is a slice computed based on Front $\left(\mathrm{TF}_{\mathrm{A}}, 1+1\right)$ and used variables at calling action $\mathrm{X}^{1+1}, \mathrm{U}\left(\mathrm{X}^{1+1}\right)$.

2) Calling-to-Called

Total slice $_{\mathrm{AB}}=$ Slice $_{\mathrm{A}} \cup \mathrm{TF}_{\mathrm{B}}$

where

(1) Slice ${ }_{A}$ is a slice computed based on Front $\left(\mathrm{TF}_{\mathrm{A}}, \mathrm{m}\right)$ and slicing criterion $\mathrm{C}=\left(\mathrm{x}, \mathrm{X}^{\mathrm{m}}, \mathrm{V}\right)$

(2) $\mathrm{TF}_{\mathrm{B}}$ is a function trajectory of function $\mathrm{B}$.

Let Calling $\left(\mathrm{X}^{\mathrm{p}}\right)$ be a set of calling functions that are used to call a called function in action $x^{p}$, where $x^{p} \in M(T)$. For example in the trajectory of Figure 21, Calling $\left(22^{16}\right)=\{\mathrm{FaC}\}$

Let Called $\left(\mathrm{X}^{\mathrm{P}}\right)$ be a set of called functions that are called by a calling function in action $X^{p}$, where $X^{p} \in M(T)$. For example in the trajectory of Figure 21 , Called $\left(5^{4}\right)=$ $\{\mathrm{Fac}\}$.

Let EI be a Called-to-Calling Relation between called and calling functions. Let $\mathrm{M}(\mathrm{T})$ be a set of actions in a given trajectory $\mathrm{T}$ of length $\mathrm{m}$. EI is a binary relation on $\mathrm{M}(\mathrm{T})$ defined as follows:

$$
\begin{aligned}
& \text { Let } T=<X_{1}, X_{2}, \ldots, X_{t}, \ldots, X_{m}>, \\
& X^{p} \text { EI } Y^{t}, t \leq p<m \text {, iff there exists function } f \\
& \text { such that }(1) \text { a called function } f \in \operatorname{Called}\left(Y^{t}\right), \\
& \text { (2) a calling function } f \in \operatorname{Calling}\left(X^{\mathrm{p}}\right) \text {, and } \\
& \text { (3) } X^{\mathrm{p}} \text { is the calling action, where the calling function } \mathrm{f} \\
& \text { at } p \text { calls a called function } f \text { at } t
\end{aligned}
$$

For example in the trajectory of Figure 21, we have $22^{16} \mathrm{EI} 5^{4}$, as shown in Figure 33. 


$$
\operatorname{EI}\left(22^{16}\right)=\left\{5^{4}\right\}
$$

Figure 33. The EI relation for the trajectory depicted in Figure 21

Let IE be a Calling-to-Called Relation between called and calling functions. Let $\mathrm{M}(\mathrm{T})$ be a set of actions in a given trajectory $\mathrm{T}$ of length $\mathrm{m}$. IE is a binary relation on $\mathrm{M}(\mathrm{T})$ defined as follows:

$$
\begin{aligned}
& \text { Let } T=<X_{1}, X_{2}, \ldots, X_{t}, \ldots, X_{m}> \\
& X^{p} \text { IE } Y^{t}, I \leq p<t, \text { iff there exists function } f \\
& \text { such that }(1) \text { a calling function } f \in \operatorname{Calling}\left(Y^{t}\right), \\
& \text { (2) a called function } f \in \operatorname{Called}\left(X^{\mathrm{p}}\right) \text {, and } \\
& \text { (3) } X^{\mathrm{p}} \text { is the called action where the called function } \mathrm{f} \\
& \text { at } \mathrm{p} \text { is called by a calling function } \mathrm{f} \text { at } t
\end{aligned}
$$

For example in the trajectory of Figure 21 , we have $5^{4} \mathrm{IE} 22^{16}$, as shown in Figure 34.

$$
\operatorname{IE}\left\{5^{4}\right\} \quad=\left\{22^{16}\right\}
$$

Figure 34. The IE relation for the trajectory depicted in Figure 21.

\section{Definition 17}

To find the slicing set $S_{C}$, we first find the set $A^{0}$ of all actions that have direct influence on $\mathrm{V}$ at $\mathrm{q}$ and on action $\mathrm{I}^{\mathrm{q}}$. $\mathrm{A}^{0}$ is defined as follows [Korel and Laski 90].

$$
A^{0}=L D(q, V) \cup L T\left(I^{q}\right) \cup I^{q}
$$

where $L D(q, V)$ is the set of last definitions of variables in $V$ at the execution position $q$, and $L T\left(I^{q}\right)$ is a set of test actions that have Test-Control influence on $I^{q}$. 
We will find $S_{c}$ iteratively, as the limit of a sequence $S^{0}, S^{1}, \ldots, S^{n}, 0 \leq n<q$, which is defined as follows.

$$
S^{0}=A^{0} \text { and } S^{i+1}=S^{i} \cup A^{i+1}
$$

where

$$
\begin{aligned}
& A^{i+1}=\left\{X^{p} \in M\left(T F_{\text {name }}\right): 1 \leq p<q,\right. \\
& \text { (1) } X^{p} \notin S^{i} \text {, and } \\
& \text { (2) there exists } \left.Y^{t} \in S^{i}, t<q, X^{p} Z Y^{t}\right\} \\
& \text { where } Z=D U \cup T C \cup I R \cup L D R
\end{aligned}
$$

Finally, we can get the slice from the following definition.

$$
S_{c}=S^{k}
$$

where $S^{k}$ is the limit of the sequence $\left\{S^{i}\right\}$.

\section{Definition 18}

Let $F N(q)$ be a string of function name such that $X^{q}, X$ is in the scope of influence. For example in Figure 21, $\mathrm{TF}_{\mathrm{Fac}}=\left\{5^{4}, 7^{5}, 8^{6}, 10^{7}, 11^{8}, 12^{9}, 13^{10}, 10^{11}, 11^{12}\right.$, $\left.12^{13}, 13^{14}, 14^{15}, 15^{16}\right\}$, then $\mathrm{FN}(8)=$ "Fac", because 11 is in the scope of influence of function name Fac. FN(17)= "main" for the same reason.

Definition 19

Let $\mathrm{G}(\mathrm{X})$ be a set of variables and precedences that are declared as a part of global declaration. $\mathrm{G}(\mathrm{X})$ is computed from the source program, not from a trajectory path. In Figure $20, \mathrm{G}(1)=\{$ include $\}$ and $\mathrm{G}(3)=\{\mathrm{FaC}\}$. 
Definition 20

Let VDU(FunctionName) be a set of variables that are used, $\mathrm{UF}_{\text {name, }}$ and defined, $\mathrm{DF}_{\text {name, }}$ in a given function name. For example, $\operatorname{VDU}(\operatorname{ma} i \mathrm{n})=\{\mathrm{Num}\}$ and $\operatorname{VDU}(\mathrm{FaC})=$ $\{F, I, N\}$ in Figure 21.

\section{Definition 21}

In order to find the scope of influence of each instruction, variable scope, VS, and control scope, CS, are used as defined bellow.

1. Variable scope, VS, gives the information that the variables that used or defined in each instruction were declared at what instructions.

Let $\mathrm{X}_{\mathrm{DCL}}$ be an instruction that declared variables such as "int $I_{;}$"”.

Let $\mathrm{X}_{\mathrm{DU}}$ be an instruction that used or defined the variables declared by $\mathrm{X}_{\mathrm{DCL}}$, where variables that are used or defined are in the scope of influence of the variables that are declared in $X_{D C L}$. For example, " $I=I+1 ;$;, which is the first $I$ is defined and the second I is used both are declared by "int I;".

Then we get $\operatorname{VS}\left(\mathrm{X}_{\mathrm{DU}}\right)$, a variable scope relation at $\mathrm{X}_{\mathrm{DU}}$, which is a set of instructions $X_{D C L}$, where $X_{D U}$ is in the scope of influence of $X_{D C L}$.

For example in Figure 20, we get $\operatorname{VS}\{11\}=\{7,8\}$ since variable $F$ at $\mathrm{X}_{\mathrm{DU}}=11$ was declared in $X_{D C L}=7$, and variable $I$ at $X_{D U}=11$ was declared in $X_{D C L}=8$. The VS relation for the program depicted in Figure 20 is shown in Figure 36.

2. Control scope, CS, gives information about instructions that are in the scope of influence of control instructions such as test statements, functions, and classes. For 
calculation of the scope of influence of each statement, the me_too set is used [Lyle 84].

Let $\mathrm{X}$ be an instruction, the me_too is a set of instructions that are in the scope of influence of instruction $\mathrm{X}$.

Due to the complexity of the $\mathrm{C}++$ language and in order for $\mathrm{C}++$ Debug to be applicable to programs containing functions, classes, namespaces, unions, structures, and preprocessors (a separate first step in compilation, e.g., \#include, \#define, or \#if), the me_too set was modified according to the rules shown in Figure 35 and will still be called the control scope, CS, set.

Based on the rules in Figure 35, Figures 36 shows an example of computing the CS set of a tested program that computes the factorial in Figure 20.

To find the final slicing set $F_{S}$ with scope, we first find the set $S^{0}$ of all instructions that sliced from the tested program $P$ based on slicing criterion $C\left(x, I^{q}, V\right)$. $\mathrm{S}^{0}$ is defined as follows.

$$
\mathrm{S}^{0}=\mathrm{S}_{\mathrm{c}}
$$

where $S_{c}$ is a slicing set defined in Definition 17 .

We will find $F_{s}$ iteratively, as the limit of a sequence $F^{0}, F^{1}, \ldots, F^{n}, 0 \leq i<n, n$ = length of program $\mathrm{P}$, which is defined as follows.

$$
F^{0}=S^{0} \text { and } F^{i+1}=F^{i} \cup S^{i+1}
$$

where

$$
\begin{aligned}
S^{i+1}= & \{X \in P: 1 \leq X<n, n=\text { length of program } P, \\
& \text { (1) } X \notin F^{i} \text {, and } \\
& \text { (2) there exists } \left.Y \in F^{i}, X \in Z(Y)\right\}
\end{aligned}
$$


1. For any straight-line instruction, the CS set must contain:

1.1 Instruction of which it is in the scope of influence

2. For any control instruction, the CS set must contain:

2.1 Instruction of which it is in the scope of influence

2.2 Instruction representing the beginning of the scope of influence

2.3 Instruction representing the end of the scope of influence

3. In case of functions, the CS set of that instruction must contain

3.1 Instruction of which it is in the scope of influence

3.2 Instruction representing the beginning of the scope of influence

3.3 Instruction representing the end of the scope of influence

4. In case of classes, structures, unions, and namespaces, the CS set of that instruction must contain

4.1 Instruction representing the beginning of the scope of influence

4.2 Instruction representing the end of the scope of influence

Figure 35. Rules for computing the CS (control scope) set

where $\mathrm{Z}=\mathrm{VS} \cup \mathrm{CS}$

Finally, we can get the final slice with scope from the following definition.

$$
F_{\mathrm{s}}=\mathrm{F}^{\mathrm{k}}
$$

where $\mathrm{F}^{\mathrm{k}}$ is the limit of the sequence $\left\{\mathrm{F}^{\mathrm{i}}\right\}$. 


\begin{tabular}{|c|c|c|c|c|c|c|c|c|}
\hline Instruction (X) & Prototype & Called & Calling & D-set & $\mathrm{U}$-set & DCL-set & VS-set & CS-set \\
\hline 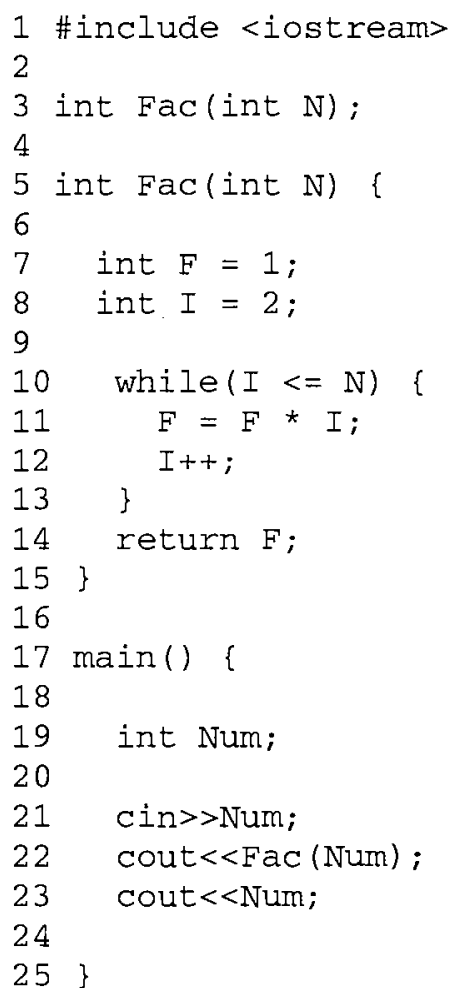 & $\operatorname{Fac}(2)$ & $\begin{array}{l}\text { Fac (2) } \\
\operatorname{main}(6)\end{array}$ & $\operatorname{Fac}(10)$ & 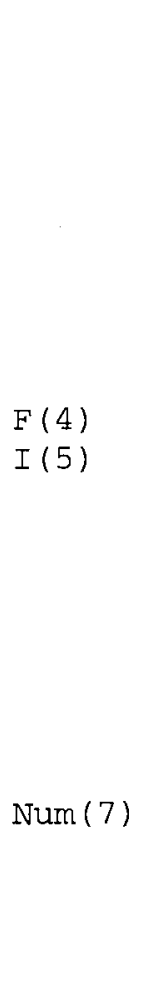 & $\begin{array}{l}N(3), I(5) \\
F(4), I(5) \\
I(5) \\
F(4) \\
\\
\operatorname{cin}(8) \\
\operatorname{Num}(7), \operatorname{cout}(9) \\
\operatorname{cout}(9), \operatorname{Num}(7)\end{array}$ & $\begin{array}{l}\text { include } \\
N(1) \\
N(3) \\
F(4) \\
I(5) \\
\end{array}$ & $\begin{array}{ll} & \\
& \\
5, & 8 \\
7, & 8 \\
8 & \\
7 & \end{array}$ & $\begin{array}{l}15 \\
5 \\
5 \\
5, \quad 13 \\
10 \\
10 \\
10 \\
5 \\
5 \\
25 \\
17 \\
17 \\
17 \\
17 \\
17\end{array}$ \\
\hline
\end{tabular}

Figure 36. The Prototype, Called, Calling, D, U, DCL, VS, and CS sets for the program depicted in Figure 20 


\subsection{Algorithms}

\subsubsection{Algorithm for Computing a Slice}

Figure 37 presents the algorithm designed and implemented for $\mathrm{C}++$ Debug. The algorithm is separated into 4 parts: Datastructures, Initialize, PASS I, and PASS II. The Datastructures part is shown in Figure 38. The Initialize part is used to initialize variables, files, etc., when the program starts.

The objectives of PASS I are to create databases and to create a trajectory T. All computations in PASS I are determined based on a source code program. The databases are used to collect the necessary information used in PASS II such as Symbol Table, List of Reserved Words, List of Basic Types, Types, Identifiers Information, Scope of Influent, etc. The trajectory $\mathrm{T}$ is created by a tool named cpptrace (for more detail see Subsection 4.3.1).

PASS II uses the information in each database and the trajectory T from PASS I to compute a set of slices. First, a slicing criterion comprising of a set of variables $\mathrm{V}$ and position $q$ is entered. After that, each slice of each variable in set $V$ at position $q$ is computed one by one. The process starts with finding a slice inside the function where position $q$ is at, until finished. Then the algorithm goes to its calling function and starts to find a slice in this calling function again. The process is repeated until the final slice of the calling function named main() is computed. Clearly, the slice of each variable in the set $\mathrm{V}$ is computed based on all functions that related to each variable in the set $\mathrm{V}$ starting from the function where position $q$ is at, its calling function, ..., and end at function main(). Compute_scope_of_influence(C) makes the final slice completed by adding some statements that may govern each statement in the slice. 


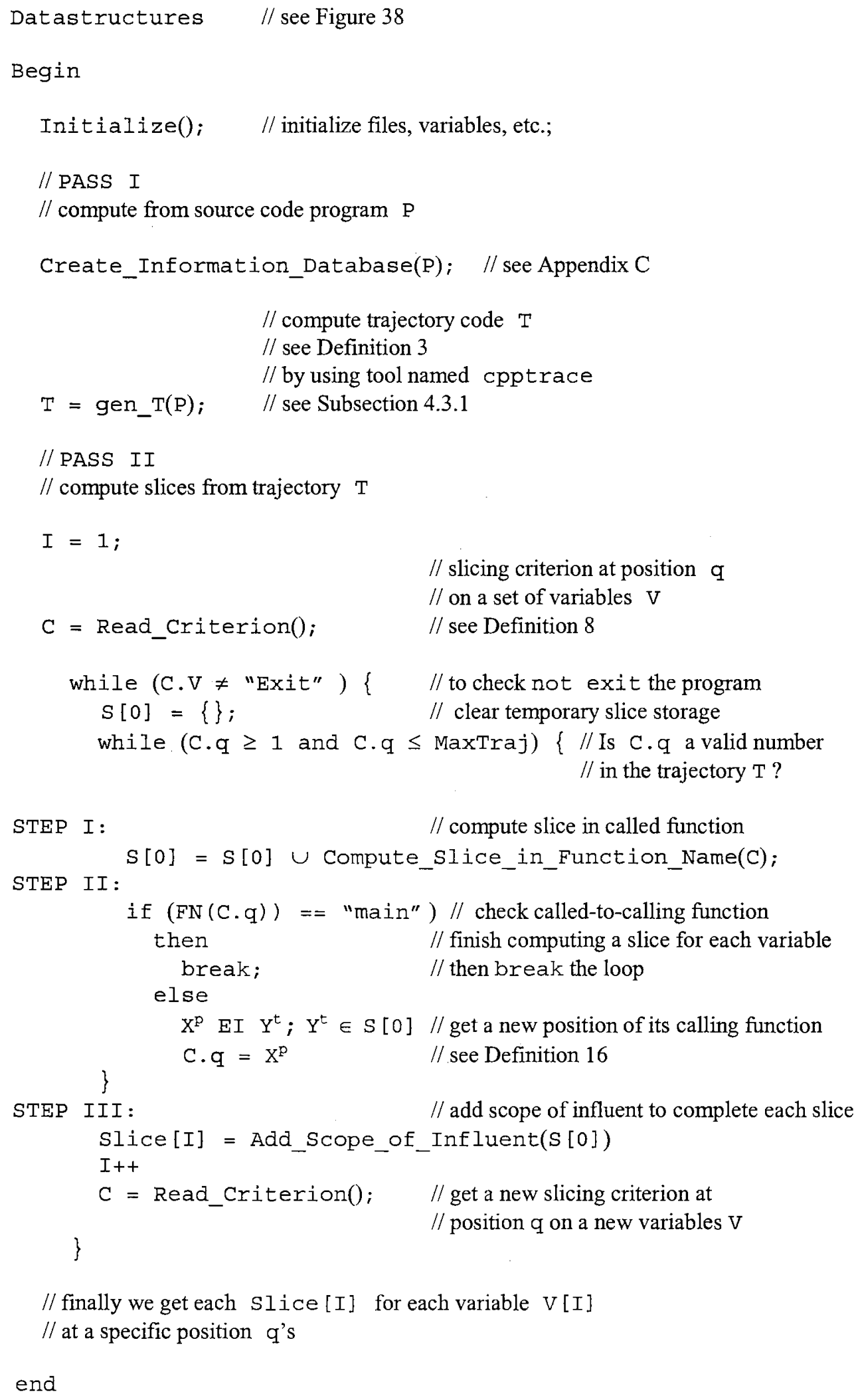

Figure 37. Algorithm to compute a set of slices 


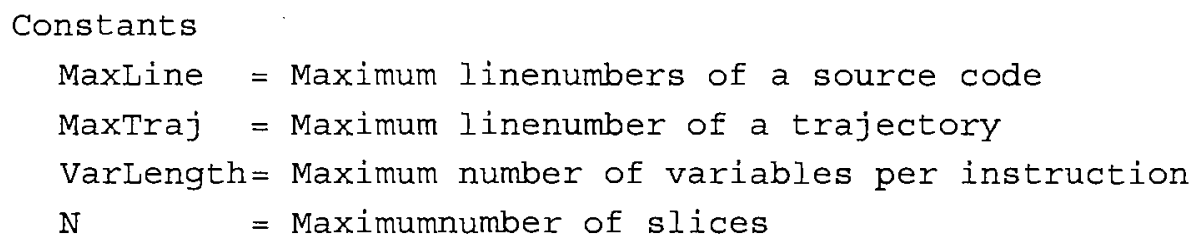

Figure 38. Slicing data structures 


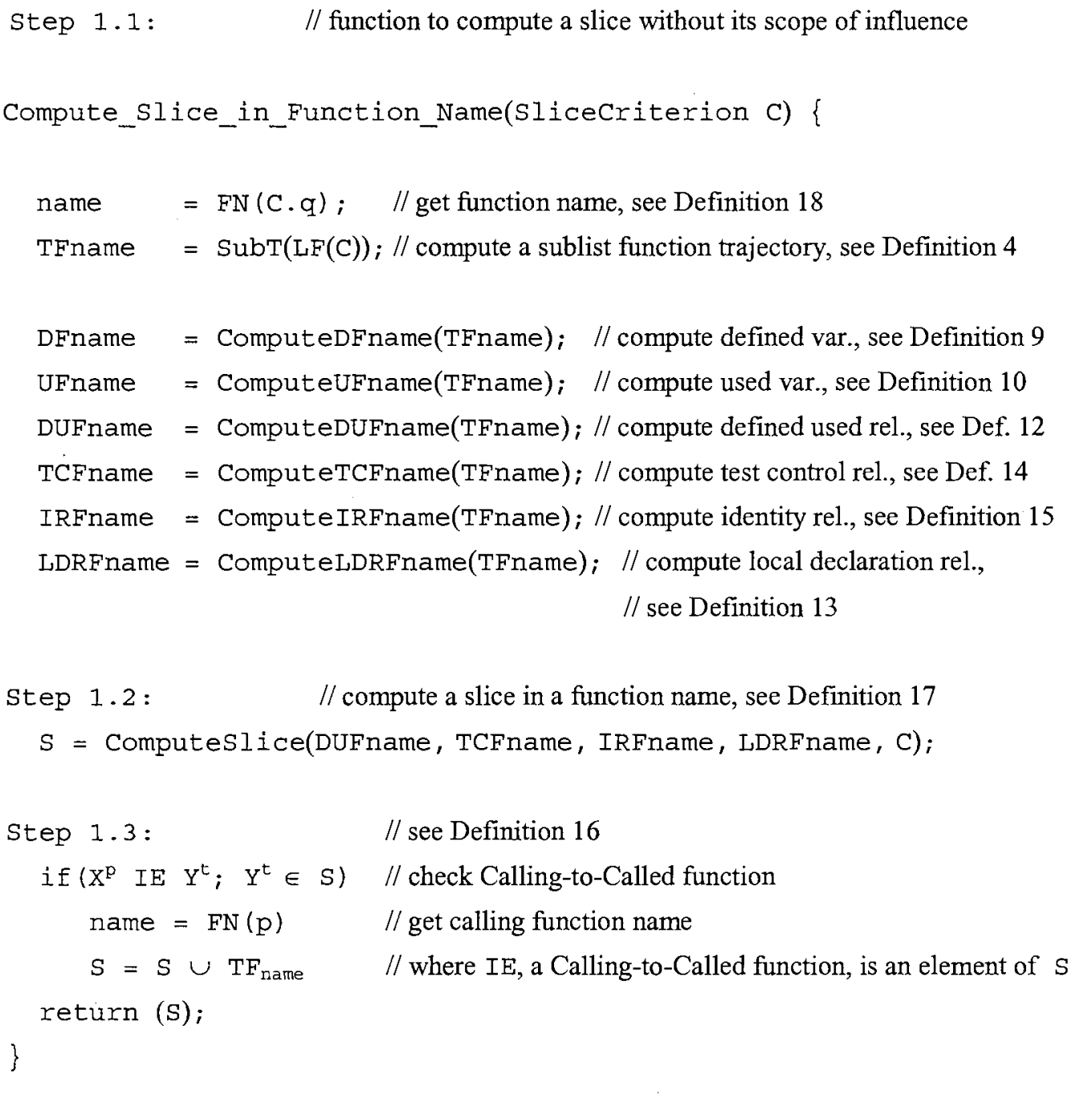

Figure 39. Algorithm to compute a slice of each function

// function to compute the scope of influence of a slice
Add_Scope_of_Influent(array [1..n] of set [action] s)
$S=$ Var_Control_Scope (s); // Add scope of influence to a slice,
// see Definition 21

Figure 40. Function to compute the scope of influence of a slice 


\subsection{Examples: How to Compute a Slice of a Program Containing Functions}

There are three examples in this section: an example of slicing a program based on variable Num (which is in the function main), an example of slicing a program based on the calling function Fac (in the case of Calling-to-Called function), and an example of slicing a program based on the calling function I (in the case of Called-to-Calling function).

Example 1. This example shows how to compute a slice based on variable Num, which is in the function main. Consider trajectory $\mathrm{T}$ in Figure 21. Using the criterion $\mathrm{C}=(\mathrm{x}$, $\left.23^{17},\{\mathrm{Num}\}\right)$, we have $\mathrm{x}=(\mathrm{Num})=(3)$.

The step-by-step trace of the algorithm in Figure 37 follows.

Step 1:

Compute $S[0]=S[0] \cup$ Compute_Slice_in_Function_Name(C)

Step 1.1: // start from Compute_Slice_in_Function_Name(C)

$$
\begin{aligned}
& \mathrm{FN}(\mathrm{C} . \mathrm{q})=\mathrm{FN}(17)=\text { "main" } \\
& \text { // therefore compute slice in function "main" } \\
& \text { compute } \mathrm{TF}_{\text {main }}=\left\{17^{1}, 19^{2}, 21^{3}, 22^{16}, 23^{17}, 25^{18}\right\} / / \text { as shown in Figure } 21 \\
& \text { compute } \mathrm{DF}_{\text {main }}, \mathrm{UF}_{\text {main }} \quad / / \text { as shown in Figure } 23 \\
& \text { compute } \mathrm{DUF} \text { main } \\
& \text { compute } \mathrm{TCF}_{\text {main }}=\{\} \quad / / \text { as shown in Figure } 25 \\
& \text { compute } \mathrm{IRF} F_{\text {main }}=\{\} \quad / / \text { as shown in Definition } 13 \\
& \text { compute } \mathrm{LDRF} \text { main }=\{\} \quad / / \text { as shown in Figure } 27
\end{aligned}
$$

Step 1.2: 
Compute $\mathrm{S}=$ ComputeSIice $\left(\mathrm{DUF}_{\text {main }}, \mathrm{TCF}_{\text {main }}, \mathrm{IRF}_{\text {main }}, \mathrm{LDRF}_{\text {main }}, \mathrm{C}\right)$

Since $C=\left(x, 23^{17},\{\right.$ Num $\left.\}\right) \quad / /$ given

$\operatorname{LD}(17,\{$ Num $\})=\left\{21^{3}\right\}, \operatorname{LT}\left(23^{17}\right)=\left\{17^{1}\right\}, I^{\mathrm{q}}=23^{17}$

$A^{0}=\left\{17^{1}, 21^{3}, 23^{17}\right\}, \quad S^{0}=\left\{17^{1}, 21^{3}, 23^{17}\right\}$,

$A^{1}=\left\{19^{2}\right\}, \quad S^{1}=\left\{17^{1}, 19^{2}, 21^{3}, 23^{17}\right\}$,

$\mathrm{A}^{2}=\{\}, \quad \mathrm{S}^{2}=\left\{17^{1}, 19^{2}, 21^{3}, 23^{17}\right\}$.

$S_{C}=S^{2}=\left\{17^{1}, 19^{2}, 21^{3}, 23^{17}\right\}$

Step 1.3: Check Calling-to-Called functions

No.

Finally, we get $S[0]=S[0] \cup S_{C}=\left\{17^{1}, 19^{2}, 21^{3}, 23^{17}\right\}$.

Step 2: Check for more Called-to-Calling functions

since $\operatorname{FN}(17)=$ "main" then no more calling functions and break.

Step 3: Add scope of influence

Slice[1] =Add_Scope_of_Influence(S[0])

Let $F^{0}=S_{0}=S[0]=\{17,19,21,23\}$,

$F^{0}=\{17,19,21,23\}, \quad S^{0}=\{17,19,21,23\}$,

$F^{1}=\{1,25\}, \quad S^{1}=\{1,17,19,21,23,25\}$,

$F^{2}=\{\}, \quad S^{2}=\{1,17,19,21,23,25\}$,

Slice[1] $=S^{2}=\{1,17,19,21,23,25\}$.

And finally, the dynamic slice is shown in Figure 41.

Example 2. This example shows how to compute a slice based on the calling function Fac (in case of Calling-to-Called function). Consider trajectory $T$ in Figure 21. Using 
1 \#include <iostream>

17 main() \{ // main program

19 int Num; // number

21 Cin $>>\mathrm{Num}$;

23 cout $<<$ Num; // calling function

$25\}$

Figure 41. A dynamic program slice computed based on variable Num in line 23 of the program in Figure 20

the criterion $C=\left(x, 22^{16},\{\operatorname{FaC}\}\right)$, we have $x=($ Num $)=(3)$.

The step-by-step trace of the algorithm in Figure 37 follows.

Step 1:

Compute $S[0]=S[0] \cup$ Compute_Slice_in_Function_Name(C)

Step 1.1: // start from Compute_Slice_in_Function_Name(C)

$F N(C . q)=F N(16)=$ "main"

// therefore compute slice in function "main"

compute $T F_{\text {main }}=\left\{17^{1}, 19^{2}, 21^{3}, 22^{16}, 23^{17}, 25^{18}\right\} / /$ as shown in Figure 21

compute $\mathrm{DF}$ main, $\mathrm{UF}_{\text {main }} \quad / /$ as shown in Figure 23

compute DUF main $\quad / /$ as shown in Figure 25

compute $\mathrm{TCF}_{\text {main }} \quad=\{\} \quad / /$ as shown in Definition 14

compute $I R F_{\text {main }} \quad=\{\} \quad / /$ as shown in Definition 15

compute $L D R F_{\text {main }}=\{\} \quad / /$ as shown in Figure 27

Step 1.2:

Compute $\mathrm{S}=$ ComputeSlice $\left(\mathrm{DUF}_{\mathrm{ma} i n}, \mathrm{TCF}_{\mathrm{main}}, I R F_{\mathrm{main}}, \mathrm{LDRF_{ \text {main } }}, \mathrm{C}\right)$

Since $C=\left(x, 22^{16},\{\mathrm{FaC}\}\right) \quad / /$ given 


$$
\begin{aligned}
& \operatorname{LD}(16,\{\text { Fac }\})=\{\}, \operatorname{LT}\left(22^{16}\right)=\left\{17^{1}\right\}, I^{\mathrm{q}}=22^{16} \\
& \mathrm{~A}^{0}=\left\{17^{1}, 22^{16}\right\}, \quad \mathrm{S}^{0}=\left\{17^{1}, 22^{16}\right\}, \\
& \mathrm{A}^{1}=\left\{19^{2}, 21^{3}\right\}, \quad \mathrm{S}^{1}=\left\{17^{1}, 19^{2}, 21^{3}, 22^{16}\right\}, \\
& \mathrm{A}^{2}=\{\}, \quad \mathrm{S}^{2}=\left\{17^{1}, 19^{2}, 21^{3}, 23^{16}\right\}, \\
& \mathrm{S}_{\mathrm{C}}=\mathrm{S}^{2}=\left\{17^{1}, 19^{2}, 21^{3}, 22^{16}\right\},
\end{aligned}
$$

Step 1.3: Check Calling-to-Called functions

Yes, because $\quad\left\{5^{4}\right\} \operatorname{IE}\left\{22^{16}\right\}$,

$$
\begin{aligned}
& \operatorname{FN}(4)=\text { "FaC" } \\
& S_{C}=S_{C} \cup T F_{F a c}, \\
& \left.T_{F a C}=<5^{4}, 7^{5}, 8^{6}, 10^{7}, 11^{8}, 12^{9}, 13^{10}, 10^{11}, 11^{12}, 12^{13}, 13^{14}, 14^{15}\right\rangle,
\end{aligned}
$$

Finally, we get $S[0]=S[0] \cup S_{C}$

$$
\begin{aligned}
= & \left\{17^{1}, 19^{2}, 21^{3}, 5^{4}, 7^{5}, 8^{6}, 10^{7}, 11^{8}, 12^{9}, 13^{10}, 10^{11}, 11^{12}, 12^{13},\right. \\
& \left.13^{14}, 14^{15}, 22^{16}\right\} .
\end{aligned}
$$

Step 2: Check for more Called-to-Calling functions

since $\mathrm{FN}(16)=$ "main" then no more calling functions and break.

Step 3: Add scope of influence

Slice[1] =Add_Scope_of_Influence(S[0])

Let $\mathrm{F}^{0}=\mathrm{S}_{0}=\mathrm{S}[0]$

$\mathrm{F}^{0}=\{5,7,8,10,11,12,13,14,17,19,21,22\}$,

$S^{0}=\{5,7,8,10,11,12,13,14,17,19,21,22\}$,

$F^{1}=\{1,3,15,25\}$,

$S^{1}=\{1,3,5,7,8,10,11,12,13,14,15,17,19,21,22,25\}$,

$\mathrm{F}^{2}=\{\}$, 


$$
\begin{aligned}
& S^{2}=\{1,3,5,7,8,10,11,12,13,14,15,17,19,21,22,25\} \\
& \text { Slice[1] }=S^{2}=\{1,3,5,7,8,10,11,12,13,14,15,17,19,21,22,25\}
\end{aligned}
$$

And finally, the dynamic slice is shown in Figure 42.

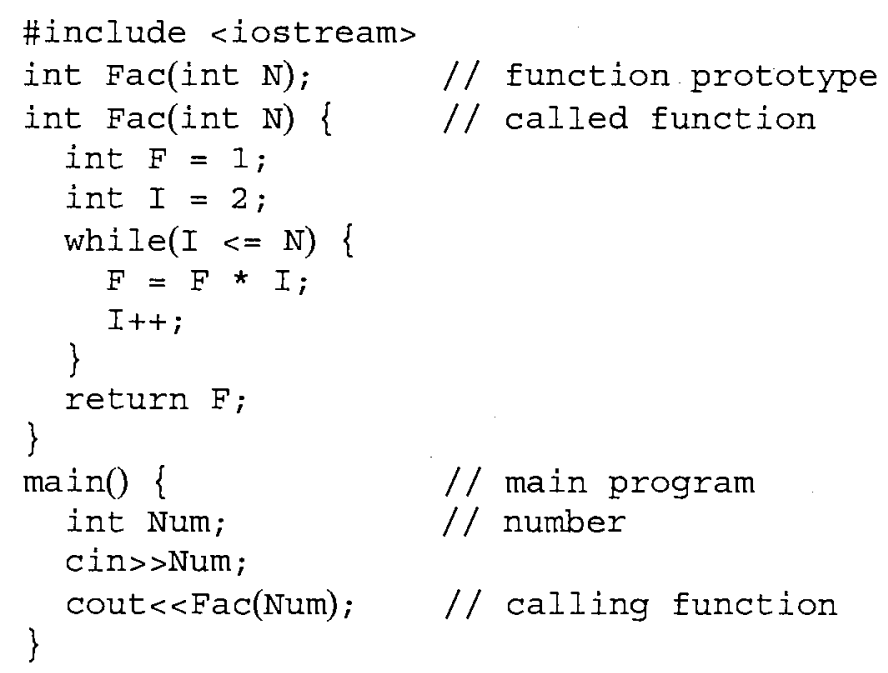

Figure 42. A dynamic program slice computed based on variable Fac in line 22 of the program in Figure 20

Example 3. This example shows how to compute a slice based on the calling function I (in case of Called-to-Calling function). Consider trajectory $T$ in Figure 21. Using the criterion $C=\left(x, 8^{6},\{I\}\right)$, we have $x=($ Num $)=(3)$.

The step-by-step trace of the algorithm in Figure 37 follows.

Step 1:

Compute $S[0]=S[0] \cup$ Compute_Slice_in_Function_Name(C)

Step 1.1: // start from Compute_Slice_in_Function_Name(C)

$$
F N(C . q)=F N(6)=" F a C "
$$


// therefore compute slice in function "Fac"

$\begin{array}{ll}\text { compute } \mathrm{TF}_{\mathrm{Fac}} & / / \text { as shown in Figure 21 } \\ \text { compute } \mathrm{DF} \mathrm{Fac}_{\mathrm{Fac}}, \mathrm{UF}_{\mathrm{Fac}} & / / \text { as shown in Figure 22 } \\ \text { compute } \mathrm{DUF} F_{\mathrm{Fac}} & \text { // as shown in Figure 24 } \\ \text { compute } \mathrm{TCF}_{\mathrm{Fac}}=\{\} & / / \text { as shown in Definition 14 } \\ \text { compute } \mathrm{IRF} \mathrm{Fac}_{\mathrm{Fac}}=\{\} & / / \text { as shown in Definition 15 } \\ \text { compute } \mathrm{LDRF} \mathrm{Fac}_{\mathrm{Fac}}=\{\} & / / \text { as shown in Figure 26 }\end{array}$

Step 1.2:

Compute $\mathrm{S}=$ ComputeSlice $\left(\mathrm{DUF}_{\mathrm{Fac}}, \mathrm{TCF}_{\mathrm{Fac}}, \mathrm{IRF}_{\mathrm{Fac}}, \mathrm{LDRF}_{\mathrm{Fac}}, \mathrm{C}\right)$

$$
\begin{aligned}
& \text { Since } C=\left(x, 8^{6},\{I\}\right) \quad \text { //given } \\
& \operatorname{LD}(6,\{I\})=\{\}, \operatorname{LT}\left(8^{6}\right)=\left\{5^{4}\right\}, I^{q}=8^{6} \\
& A^{0}=\left\{5^{4}, 8^{6}\right\}, \quad S^{0}=\left\{5^{4}, 8^{6}\right\}, \\
& A^{1}=\{\}, \\
& S_{C}=S^{1}=\left\{5^{4}, 8^{6}\right\} .
\end{aligned}
$$

Step 1.3: Check Calling-to-Called functions

No.

Finally, we get $S[0]=S[0] \cup S_{C}=\left\{5^{4}, 8^{6}\right\}$.

Step 2: Check for more Called-to-Calling functions since $\mathrm{FN}(6)=$ "Fac", there is more calling functions since $22^{16}$ EI $5^{4}$, then C. $q=16$; Go to Step 1

Step 1:

Compute $S[0]=S[0] \cup$ Compute_Slice_in_Function_Name(C)

Step 1.1: // start from Compute_Slice_in_Function_Name(C) 
$\mathrm{FN}(\mathrm{C} . \mathrm{q})=\mathrm{FN}(16)=$ "ma in"

// therefore compute slice in function "main"

compute $T F_{\text {main }}=\left\{17^{1}, 19^{2}, 21^{3}, 22^{16}, 23^{17}, 25^{18}\right\} / /$ as shown in Figure 21

compute $\mathrm{DF}_{\text {main }}, \mathrm{UF}_{\text {main }} \quad / /$ as shown in Figure 23

compute $\mathrm{DUF}_{\text {main }} \quad / /$ as shown in Figure 25

compute $\mathrm{TCF}_{\text {main }}=\{\} \quad / /$ as shown in Definition 14

compute $I R F_{\text {main }}=\{\} \quad / /$ as shown in Definition 15

compute $L D R F_{\text {main }}=\{\} / /$ as shown in Figure 26

Step 1.2:

Compute $\mathrm{S}=$ Computeslice $\left(\mathrm{DUF}_{\text {main }}, \mathrm{TCF}_{\text {main }}, \mathrm{IRF}_{\text {main }}, \mathrm{LDRF}_{\text {main }}, \mathrm{C}\right)$

Since $C=\left(x, 22^{16},\{\mathrm{FaC}\}\right) \quad / /$ given

$$
\begin{aligned}
& \operatorname{LD}(16,\{\text { Fac }\})=\{\}, \operatorname{LT}\left(22^{16}\right)=\left\{17^{1}\right\}, I^{\mathrm{q}}=22^{16} \\
& \mathrm{~A}^{0}=\left\{17^{1}, 22^{16}\right\}, \quad \mathrm{S}^{0}=\left\{17^{1}, 22^{16}\right\}, \\
& \mathrm{A}^{1}=\left\{19^{2}, 21^{3}\right\}, \quad \mathrm{S}^{1}=\left\{17^{1}, 19^{2}, 21^{3}, 22^{16}\right\}, \\
& \mathrm{A}^{2}=\{\}, \quad \mathrm{S}^{2}=\left\{17^{1}, 19^{2}, 21^{3}, 23^{16}\right\}, \\
& \mathrm{S}_{\mathrm{C}}=\mathrm{S}^{2}=\left\{17^{1}, 19^{2}, 21^{3}, 22^{16}\right\} .
\end{aligned}
$$

Step 1.3: Check Calling-to-Called functions

No.

Finally, we get $S[0]=S[0] \cup S_{C}=\left\{21^{3}, 5^{4}, 8^{6}, 22^{16}\right\}$.

Step 2: Check for more Called-to-Calling functions

since $F N(16)=$ "main", no more calling functions and break.

Step 3: Add scope of influence

Slice[1] = Add_Scope_of_Influence(S[0]) 


$$
\begin{aligned}
& \text { Let } F^{0}=S_{0}=S[0]=\{5,8,17,19,21,22\}, \\
& F^{0}=\{5,8,17,19,21,22\}, \\
& S^{0}=\{5,8,17,19,21,22\}, \\
& F^{1}=\{1,3,15,25\}, \\
& S^{1}=\{1,3,5,8,15,17,19,21,22,25\}, \\
& F^{2}=\{\}, \\
& S^{2}=\{1,3,5,8,15,17,19,21,22,25\}, \\
& \text { Slice }[1]=S^{2}=\{1,3,5,8,15,17,19,21,22,25\} .
\end{aligned}
$$

And finally, the dynamic slice is shown in Figure 43.

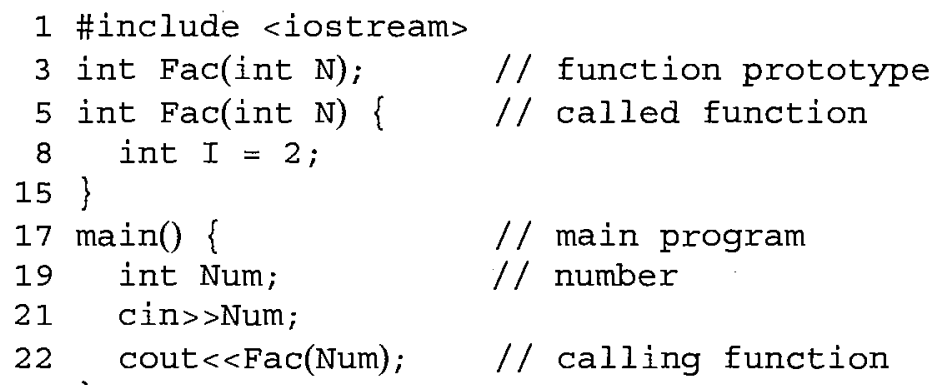

Figure 43. A dynamic program slice computed based on variable $I$ in line 8 of the program in Figure 20

\subsection{A Slice with Classes, Structures, and Unions}

A class contains members, variables, and functions. Each slice of the member functions is computed in the same way as a normal function mentioned in Section 3.4. After a slice of a member function is computed, the rest of the slice code in the class is determined by variable scope VS and control scope CS. The VS and CS sets are the key to obtaining a slice program of a program with classes. A slice of a program with 
Structures and Unions is computed the same way as a slice of a program with classes, since all have the same grammar structures.

The program in Figure 44 computes the sum and average of integers. In this example, variable Max is 4 and the array called Num contains 10.0, 20.0, 15.0, and 5.0. Upon completion of program execution, the program should yield one results as 12.5 . However, this program contains an error in line 24. Rather than return Sum()/Max, the program computes return $\operatorname{Sum}() /(\operatorname{Max}+1)$, thus yielding an error (Avg $=10.0$ instead of 12.5). To localize such an error, program slicing and dicing techniques can be used. The trajectory of the program in Figure 44 is shown in Figure 45.

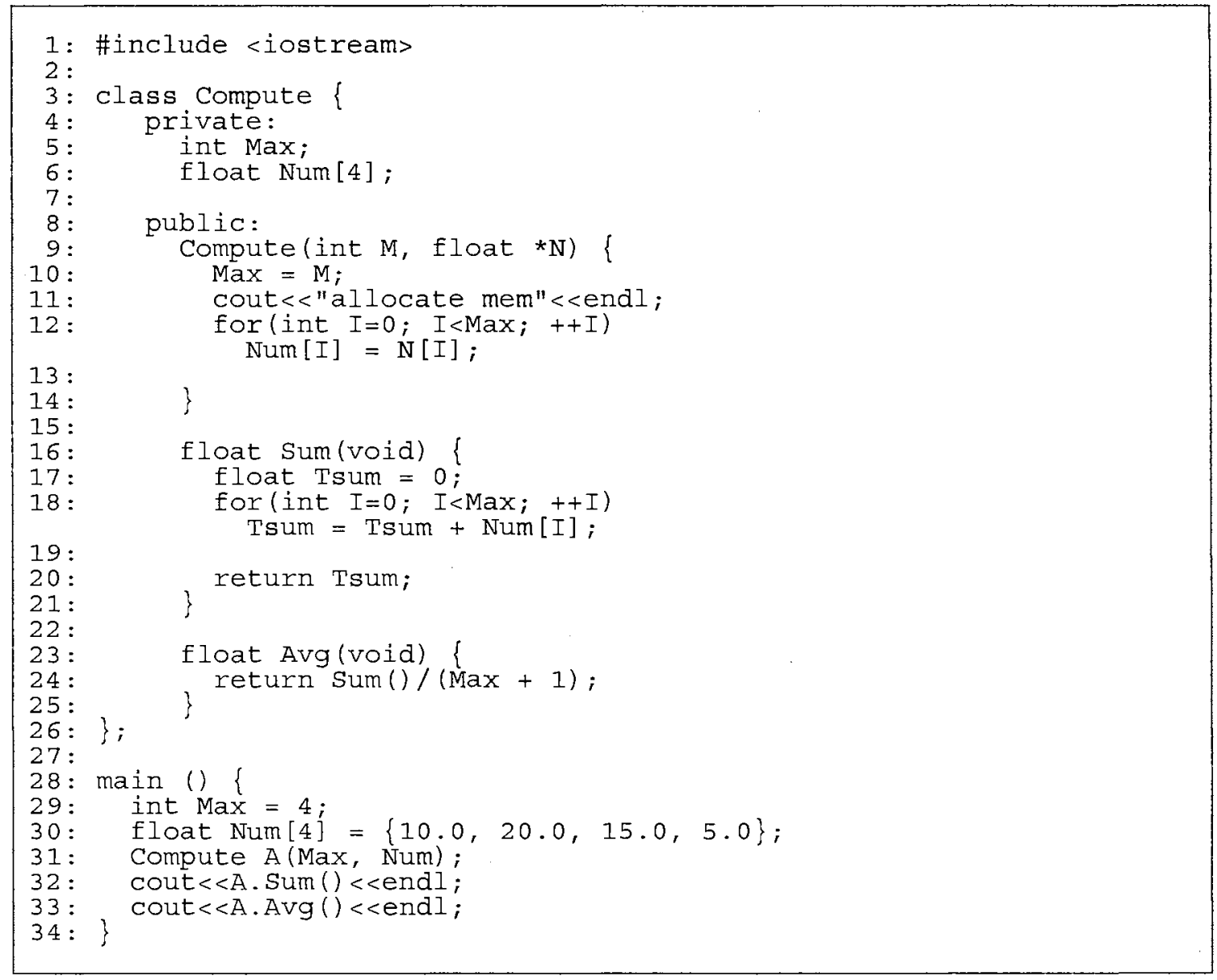

Figure 44. A program for calculating the sum and average of a set of numbers 


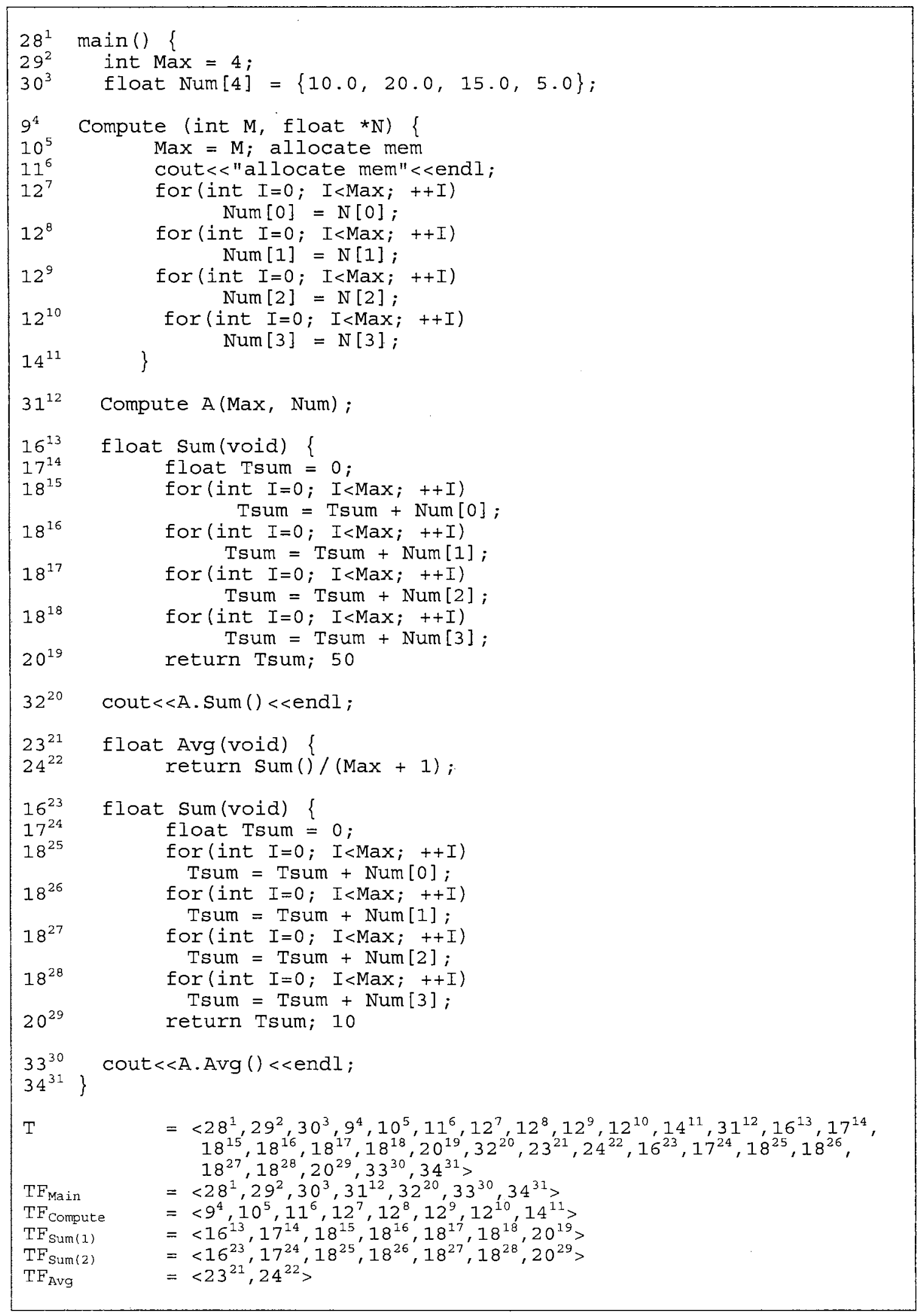

Figure 45 . The trajectory of the program from Figure 44 on input data $\operatorname{Max}=4$, Num $=(10.0,20.0,15.0,5.0)$ 


\begin{tabular}{|c|c|c|c|c|c|c|c|c|}
\hline Instruction $(\mathrm{X})$ & Prototype & Called & Calling & D-set & U-set & DCL-set & VS-set & CS-set \\
\hline 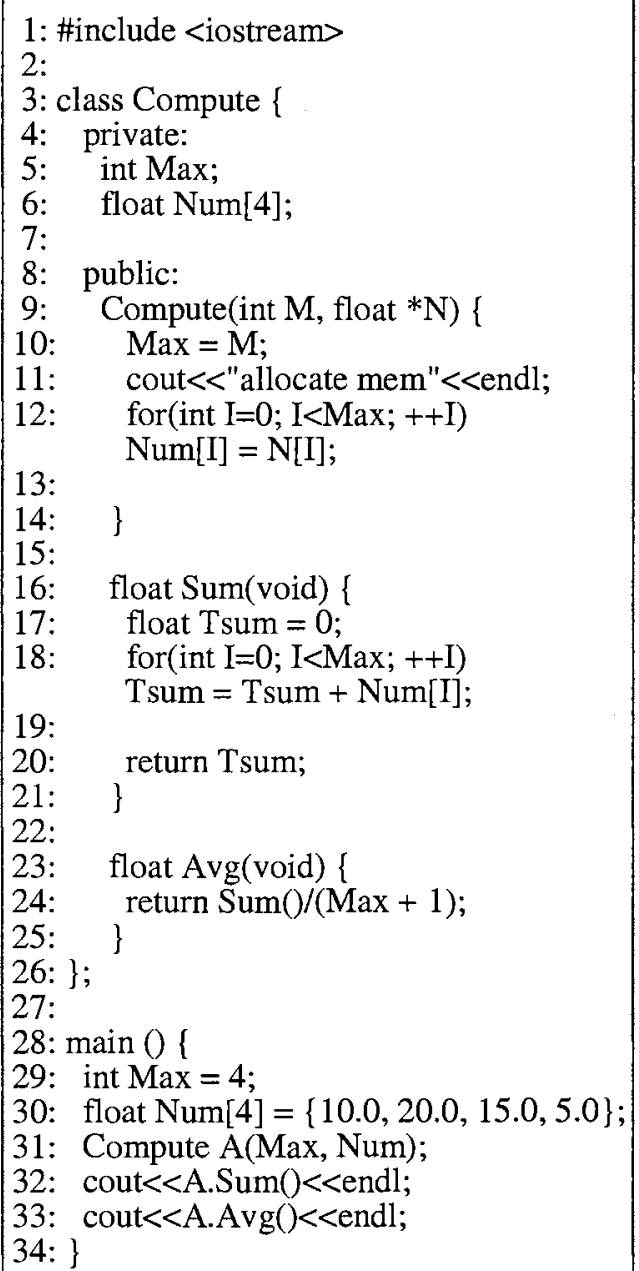 & Compute $(01)$ & $\begin{array}{l}\operatorname{Avg}(13) \\
\operatorname{main}(14)\end{array}$ & Sum(10) & $\begin{array}{l}\operatorname{Max}(02) \\
\operatorname{Num}(03) \\
\mathrm{I}(09)\end{array}$ & 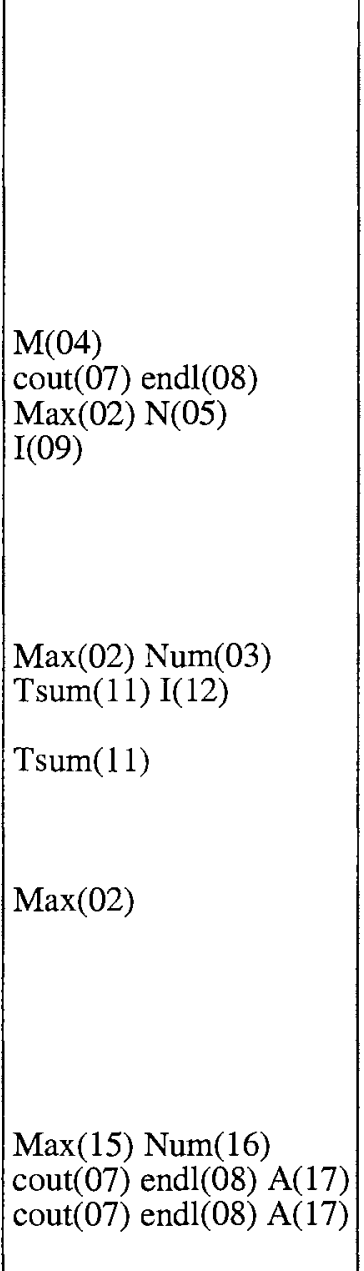 & $\begin{array}{l}\text { include } \\
\text { Max(02) } \\
\text { Num(03) } \\
\text { M(04) N(05) } \\
\text { I(09) }\end{array}$ & $\mid \begin{array}{l}9,29,30 \\
31 \\
31\end{array}$ & \begin{tabular}{|l}
26 \\
3 \\
3 \\
\\
3,14 \\
9 \\
9 \\
9 \\
9 \\
9 \\
9 \\
3,21 \\
16 \\
16 \\
\\
16 \\
16 \\
3,25 \\
23 \\
23 \\
3 \\
34 \\
28 \\
28 \\
28 \\
28 \\
28 \\
28
\end{tabular} \\
\hline
\end{tabular}

Figure 46. The Prototype, Called, Calling, D, U, DCL, VS, and CS sets for the program depicted in Figure 44 


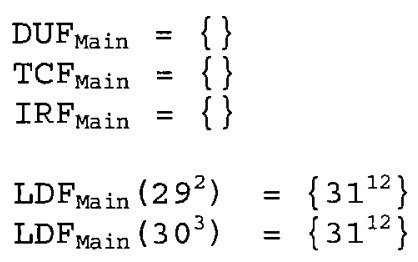

Figure 47. The DUF Main, $\mathrm{TCF}_{\text {Main }} \mathrm{LDF}_{\text {Main }}$, and $\mathrm{IRF}_{\text {Main }}$ relations that are called by $32^{20}$ for the trajectory depicted in Figure 45

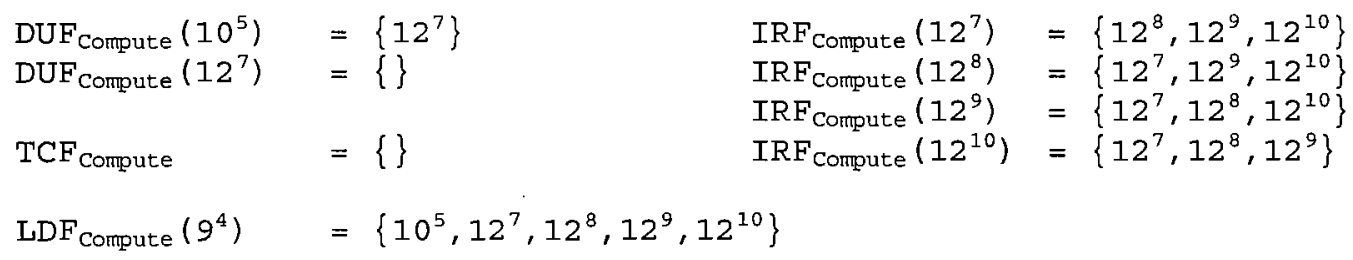

Figure 48. The DUF Compute, $\mathrm{TCF}_{\text {Compute }} \mathrm{LDF}_{\text {Compute, }}$ and $\mathrm{IRF}_{\text {Compute }}$ relations that are called by $32^{20}$ for the trajectory depicted in Figure 45

\begin{tabular}{llll|}
\hline $\operatorname{DUF}_{\text {Sum }}\left(18^{15}\right)$ & $=\left\{18^{16}\right\}$ & $\operatorname{IRF}_{\text {Sum }}\left(18^{15}\right)$ & $=\left\{18^{16}, 18^{17}, 18^{18}\right\}$ \\
$\operatorname{DUF}_{\text {Sum }}\left(18^{16}\right)$ & $=\left\{18^{17}\right\}$ & $\operatorname{IRF}_{\text {Sum }}\left(18^{16}\right)$ & $=\left\{18^{15}, 18^{17}, 18^{18}\right\}$ \\
$\operatorname{DUF}_{\text {Sum }}\left(18^{17}\right)$ & $=\left\{18^{18}\right\}$ & $\operatorname{IRF}_{\text {Sum }}\left(18^{17}\right)$ & $=\left\{18^{15}, 18^{16}, 18^{18}\right\}$ \\
$\operatorname{DUF}_{\text {Sum }}\left(18^{18}\right)$ & $=\left\{20^{19}\right\}$ & $\operatorname{IRF}_{\text {Sum }}\left(18^{18}\right)$ & $=\left\{18^{15}, 18^{16}, 18^{17}\right\}$ \\
$\operatorname{TCF}_{\text {Sum }}$ & $=\{\}$ \\
$\operatorname{LDF}_{\text {Sum }}\left(17^{14}\right)$ & $=\left\{18^{15}, 18^{16}, 18^{17}, 18^{18}, 20^{19}\right\}$
\end{tabular}

Figure 49. The $D U F_{\text {Sum }}, T C F_{\text {Sum }}, \mathrm{LDF}_{\text {Sum }}$, and $I R F_{\text {Sum }}$ relations that are called by $32^{20}$ for the trajectory depicted in Figure 45

$$
\begin{array}{rlrl}
\operatorname{DUF}_{\text {Sum }}\left(18^{25}\right) & =\left\{18^{26}\right\} & \operatorname{IRF}_{\text {Sum }}\left(18^{25}\right) & =\left\{18^{26}, 18^{27}, 18^{28}\right\} \\
\operatorname{DUF}_{\text {Sum }}\left(18^{26}\right) & =\left\{18^{27}\right\} & \operatorname{IRF}_{\text {Sum }}\left(18^{26}\right) & =\left\{18^{25}, 18^{27}, 18^{28}\right\} \\
\operatorname{DUF}_{\text {Sum }}\left(18^{27}\right) & =\left\{18^{28}\right\} & \operatorname{IRF}_{\text {Sum }}\left(18^{27}\right) & =\left\{18^{25}, 18^{26}, 18^{28}\right\} \\
\operatorname{DUF}_{\text {Sum }}\left(18^{28}\right) & =\left\{20^{29}\right\} & \operatorname{IRF}_{\text {Sum }}\left(18^{28}\right) & =\left\{18^{25}, 18^{25}, 18^{27}\right\} \\
\operatorname{TCF}_{\text {Sum }} & =\{\} & \\
\operatorname{LDF}_{\text {Sum }}\left(17^{24}\right) & =\left\{18^{25}, 18^{26}, 18^{27}, 18^{28}, 20^{29}\right\}
\end{array}
$$

Figure 50. The $\mathrm{DUF}_{\text {Sum }}, \mathrm{TCF}_{\text {Sum }} \mathrm{LDF}_{\mathrm{Sum}}$, and IRF $\mathrm{Fum}_{\text {Sum }}$ relations that are called by $24^{22}$ for the trajectory depicted in Figure 45 


$$
\begin{array}{ll}
\mathrm{DUF}_{\text {Avg }} & =\{\} \\
\mathrm{TCF}_{\text {Avg }} & =\{\} \\
\mathrm{IRF}_{\text {Avg }} & =\{\}
\end{array}
$$

Figure 51. The $D U F_{A v g}, T C F_{A v g}$, and $I R F_{A v g}$ relations for the trajectory depicted in Figure 45

Example 4. Consider trajectory $T$ in Figure 45. Using the criterion $C=\left(x, 33^{30},\{\right.$ Avg $\left.\}\right)$, we have $x=(\operatorname{Max}$, Num $)=(3,(10.0,20.0,15.0,5.0))$.

The step-by-step trace of the algorithm in Figure 37 follows.

\section{Step 1:}

Compute $S[0]=S[0] \cup$ Compute_Slice_in_Function_Name(C)

Step 1.1: // start from Compute_Slice_in_Function_Name(C) $F N(C . q)=F N(30)=$ "main"

// therefore compute slice in function "main"

$$
\begin{aligned}
& \text { compute } \mathrm{TF}_{\text {main }} \quad / / \text { as shown in Figure } 47 \\
& \text { compute } L D F_{\text {main }} \quad \text { // as shown in Figure } 47 \\
& \text { compute DUF main } \quad / / \text { as shown in Figure } 47 \\
& \text { compute } \mathrm{TCF}_{\text {main }}=\{\} / / \text { as shown in Definition } 47 \\
& \text { compute } I R F_{\text {main }}=\{\} \quad / / \text { as shown in Definition } 47 \\
& \text { compute LDRF main }=\{\} / / \text { as shown in Definition } 47
\end{aligned}
$$

Step 1.2:

Compute $\mathrm{S}=$ ComputeSIice( $\left.\mathrm{DUF}_{\text {main }}, \mathrm{TCF}_{\text {main }}, \mathrm{IRF}_{\text {main }}, \mathrm{LDRF}_{\text {main }}, \mathrm{C}\right)$ Since $C=\left(x, 33^{30},\{\mathrm{Avg}\}\right) \quad / /$ given 


$$
\begin{array}{ll}
\operatorname{LD}(30,\{\text { Avg }\})=\{\}, \operatorname{LT}\left(33^{30}\right)=\left\{28^{1}\right\}, I^{\mathrm{q}}=33^{30} \\
\mathrm{~A}^{0}=\left\{28^{1}, 33^{30}\right\}, & \mathrm{S}^{0}=\left\{28^{1}, 33^{30}\right\}, \\
\mathrm{A}^{1}=\left\{31^{12}\right\}, & \mathrm{S}^{1}=\left\{28^{1}, 31^{12}, 33^{30}\right\}, \\
\mathrm{A}^{2}=\left\{29^{2}, 30^{3}\right\}, & \mathrm{S}^{2}=\left\{28^{1}, 29^{2}, 30^{3}, 31^{12}, 33^{30}\right\}, \\
\mathrm{A}^{3}=\{\}, & \mathrm{S}^{3}=\left\{28^{1}, 29^{2}, 30^{3}, 31^{12}, 33^{30}\right\}, \\
\mathrm{S}_{\mathrm{C}}=\mathrm{S}^{3}=\left\{28^{1}, 29^{2}, 30^{3}, 31^{12}, 33^{30}\right\} .
\end{array}
$$

\section{Step 1.3: Check Calling-to-Called functions}

Yes, since $\left\{23^{21}\right\}$ IE $\left\{33^{30}\right\}$, and $\left\{9^{4}\right\}$ IE $\left\{31^{12}\right\}$,

$$
\begin{aligned}
& \mathrm{FN}(4)=\text { "Compute", and } \mathrm{FN}(21)=\text { "Avg", } \\
& \mathrm{S}_{\mathrm{C}}=\mathrm{S}_{\mathrm{C}} \cup \mathrm{TF}_{\text {Compute }} \cup \mathrm{TF}_{\text {Avg }}, \\
& \left.\mathrm{TF}_{\text {Compute }}=<9^{4}, 10^{5}, 11^{6}, 12^{7}, 12^{8}, 12^{9}, 12^{10}, 14^{11}\right\rangle, \\
& \mathrm{TF}_{\text {Avg }}=<23^{21}, 24^{22}> \\
& \mathrm{S}_{\mathrm{C}}=\left\{28^{1}, 29^{2}, 30^{3}, 9^{4}, 10^{5}, 11^{6}, 12^{7}, 12^{8}, 12^{9}, 12^{10}, 14^{11}, 31^{12},\right. \\
& \left.\quad 23^{21}, 24^{22}, 33^{30}\right\},
\end{aligned}
$$$$
\text { since }\left\{16^{23}\right\} \text { IE }\left\{24^{22}\right\} \text {, }
$$$$
\operatorname{FN}(23)=\text { "Sum", }
$$$$
\mathrm{S}_{\mathrm{C}}=\mathrm{S}_{\mathrm{C}} \cup \mathrm{TF} \mathrm{F}_{\mathrm{Sum}}
$$$$
\mathrm{TF}_{\text {Sum }}=\left\langle 16^{23}, 17^{24}, 18^{25}, 18^{26}, 18^{27}, 18^{28}, 20^{29}\right\rangle \text {, }
$$

Finally, we get $S[0]=S[0] \cup S_{c}$

$$
\begin{aligned}
= & \left\{28^{1}, 29^{2}, 30^{3}, 9^{4}, 10^{5}, 11^{6}, 12^{7}, 12^{8}, 12^{9}, 12^{10}, 14^{11}, 31^{12}, 23^{21}, 24^{22},\right. \\
& \left.16^{23}, 17^{24}, 18^{25}, 18^{26}, 18^{27}, 18^{28}, 20^{29}, 33^{30}\right\} .
\end{aligned}
$$

Step 2: Check for more Called-to-Calling functions

$$
\text { since } \mathrm{FN}(30)=\text { "ma in" then no more calling functions and break. }
$$


Step 3: Add scope of influence

$$
\begin{aligned}
& \text { Slice[1] Add_Scope_of_Influence(S[0]) } \\
& \text { Let } F^{0}=S_{0}=S[0] \\
& F^{0}=\{9,10,11,12,14,16,17,18,20,23,24,28,29,30,31,33\} \\
& S^{0}=\{9,10,11,12,14,16,17,18,20,23,24,28,29,30,31,33\}, \\
& F^{1}=\{1,3,5,6,21,25,34\}, \\
& S^{1}=\{1,3,5,6,9,10,11,12,14,16,17,18,20,21,23,24,25,28,29,30, \\
& \quad 31,33,34\}, \\
& F^{2}=\{26\}, \\
& S^{2}=\{1,3,5,6,9,10,11,12,14,16,17,18,20,21,23,24,25,26,28,29, \\
& \quad 30,31,33,34\}, \\
& F^{3}=\{\}, \\
& S^{3}=\{1,3,5,6,9,10,11,12,14,16,17,18,20,21,23,24,25,26,28,29, \\
& \quad 30,31,33,34\}, \\
& \quad 26,28,29,30,31,33,34\} .
\end{aligned}
$$

And finally, the dynamic slice is shown in Figure 52.

Example 5. Consider trajectory $\mathrm{T}$ in Figure 45 . Using the criterion $\mathrm{C}=\left(\mathrm{x}, 32^{20}\right.$, $\{$ Sum $\left.\}\right)$, we have $x=(\operatorname{Max}, N u m)=(3,(10.0,20.0,15.0,5.0))$.

The step-by-step trace of the algorithm in Figure 37 follows.

Step 1:

Compute $S[0]=S[0] \cup$ Compute_SIice_in_Function_Name(C) 


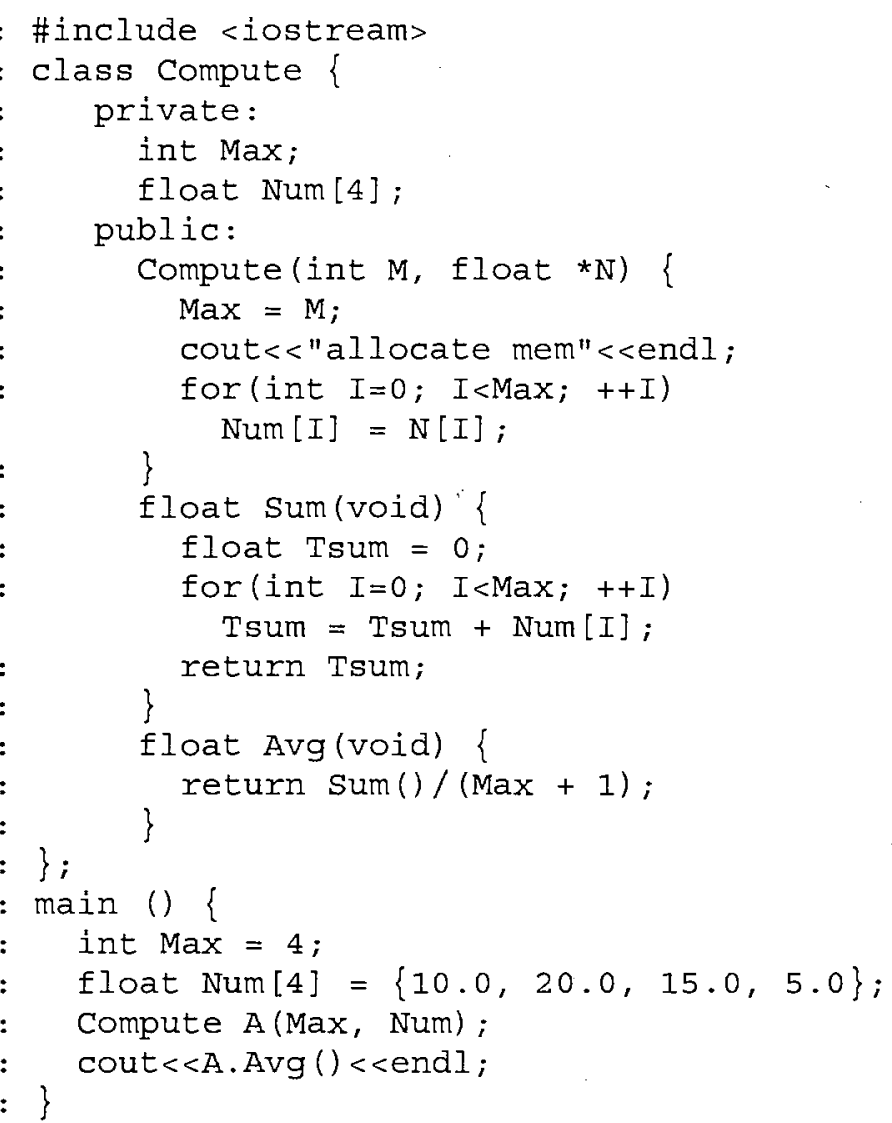

Figure 52. A dynamic program slice computed based on variable Avg in line 33 of the program in Figure 44

Step 1.1: // start from Compute_SIice_in_Function_Name(C)

$$
\begin{aligned}
& F N(C . q)=F N(20)=\text { "main" } \\
& \text { // therefore compute slice in function "main" } \\
& \text { compute } \mathrm{TF}_{\text {main }} \quad \text { // as shown in Figure } 47 \\
& \text { compute } \mathrm{LDF}_{\text {main }} \quad \text { // as shown in Figure } 47 \\
& \text { compute DUF main } \quad \text { // as shown in Figure } 47 \\
& \text { compute } \mathrm{TCF}_{\text {main }}=\{\} / / \text { as shown in Definition } 47 \\
& \text { compute IRF main }=\{\} \quad / / \text { as shown in Definition } 47
\end{aligned}
$$


compute LDRF main $=\{\} \quad / /$ as shown in Definition 47

Step 1.2:

Compute $S=$ Computeslice $\left(D U F_{\text {main }}, T C F_{\text {main }}, I R F_{\text {main }}, L D R F_{\text {main }}, C\right)$

Since $C=\left(x, 32^{20},\{\right.$ Sum $\left.\}\right) \quad / /$ given

$\mathrm{LD}(20,\{\operatorname{Avg}\})=\{\}, \operatorname{LT}\left(32^{20}\right)=\left\{28^{1}\right\}, \mathrm{I}^{\mathrm{q}}=32^{20}$

$A^{0}=\left\{28^{1}, 32^{20}\right\}, \quad S^{0}=\left\{28^{1}, 32^{20}\right\}$,

$A^{1}=\left\{31^{12}\right\}, \quad S^{1}=\left\{28^{1}, 31^{12}, 16^{13}, 32^{20}\right\}$,

$\mathrm{A}^{2}=\left\{29^{2}, 30^{3}\right\}, \quad \mathrm{S}^{2}=\left\{28^{1}, 29^{2}, 30^{3}, 31^{12}, 32^{20}\right\}$,

$A^{3}=\{\}, \quad S^{3}=\left\{28^{1}, 29^{2}, 30^{3}, 31^{12}, 32^{20}\right\}$,

$\mathrm{S}_{\mathrm{C}}=\mathrm{S}^{3}=\left\{28^{1}, 29^{2}, 30^{3}, 31^{12}, 32^{20}\right\}$.

Step 1.3: Check Calling-to-Called functions

Yes, since $\left\{9^{4}\right\}$ IE $\left\{31^{12}\right\}$, and $\left\{16^{13}\right\}$ IE $\left\{32^{20}\right\}$,

$$
\begin{aligned}
& F N(4)=\text { "Compute", and FN(13)= "Sum", } \\
& S_{C}=S_{c} \cup T F_{\text {Compute }} \cup T F_{\text {Sum, }} \\
& T F_{\text {Compute }}=<9^{4}, 10^{5}, 11^{6}, 12^{7}, 12^{8}, 12^{9}, 12^{10}, 14^{11}>, \\
& T F_{\text {Sum }}=<16^{23}, 17^{24}, 18^{25}, 18^{26}, 18^{27}, 18^{28}, 20^{29}>, \\
& S_{C}=\left\{28^{1}, 29^{2}, 30^{3}, 9^{4}, 10^{5}, 11^{6}, 12^{7}, 12^{8}, 12^{9}, 12^{10}, 14^{11}, 31^{12},\right. \\
& \left.\quad 16^{23}, 17^{24}, 18^{25}, 18^{26}, 18^{27}, 18^{28}, 20^{29}, 32^{20}\right\},
\end{aligned}
$$

Finally, we get $S[0]=S[0] \cup S_{C}$

$$
\begin{aligned}
= & \left\{28^{1}, 29^{2}, 30^{3}, 9^{4}, 10^{5}, 11^{6}, 12^{7}, 12^{8}, 12^{9}, 12^{10}, 14^{11}, 31^{12},\right. \\
& \left.16^{23}, 17^{24}, 18^{25}, 18^{26}, 18^{27}, 18^{28}, 20^{29}, 32^{20}\right\} .
\end{aligned}
$$

Step 2: Check for more Called-to-Calling functions since $\mathrm{FN}(20)=$ "main" then no more calling function and break

Step 3: Add scope of influence

Slice[1] =Add_Scope_of_Influence(S[0])

Let $\mathrm{F}^{0}=\mathrm{S}_{0}=\mathrm{S}[0]$ 


$$
\begin{aligned}
& F^{0}=\{9,10,11,12,14,16,17,18,20,28,29,30,31,32\} \\
& S^{0}=\{9,10,11,12,14,16,17,18,20,28,29,30,31,32\}, \\
& F^{1}=\{1,3,5,6,21,34\}, \\
& S^{3}=\{1,3,5,6,9,10,11,12,14,16,17,18,20,21,28,29,30,31,32,34\}, \\
& F^{2}=\{26\}, \\
& S^{2}=\{1,3,5,6,9,10,11,12,14,16,17,18,20,21,26,28,29,30,31,32,34\}, \\
& F^{3}=\{\}, \\
& S^{3}=\{1,3,5,6,9,10,11,12,14,16,17,18,20,21,26,28,29,30,31,32,34\}, \\
& \text { Slice }[1]=S^{3}=\{1,3,5,6,9,10,11,12,14,16,17,18,20,21,26,28,29,30, \\
& \quad 31,32,34\} .
\end{aligned}
$$

And finally, the dynamic slice is shown in Figure 53.

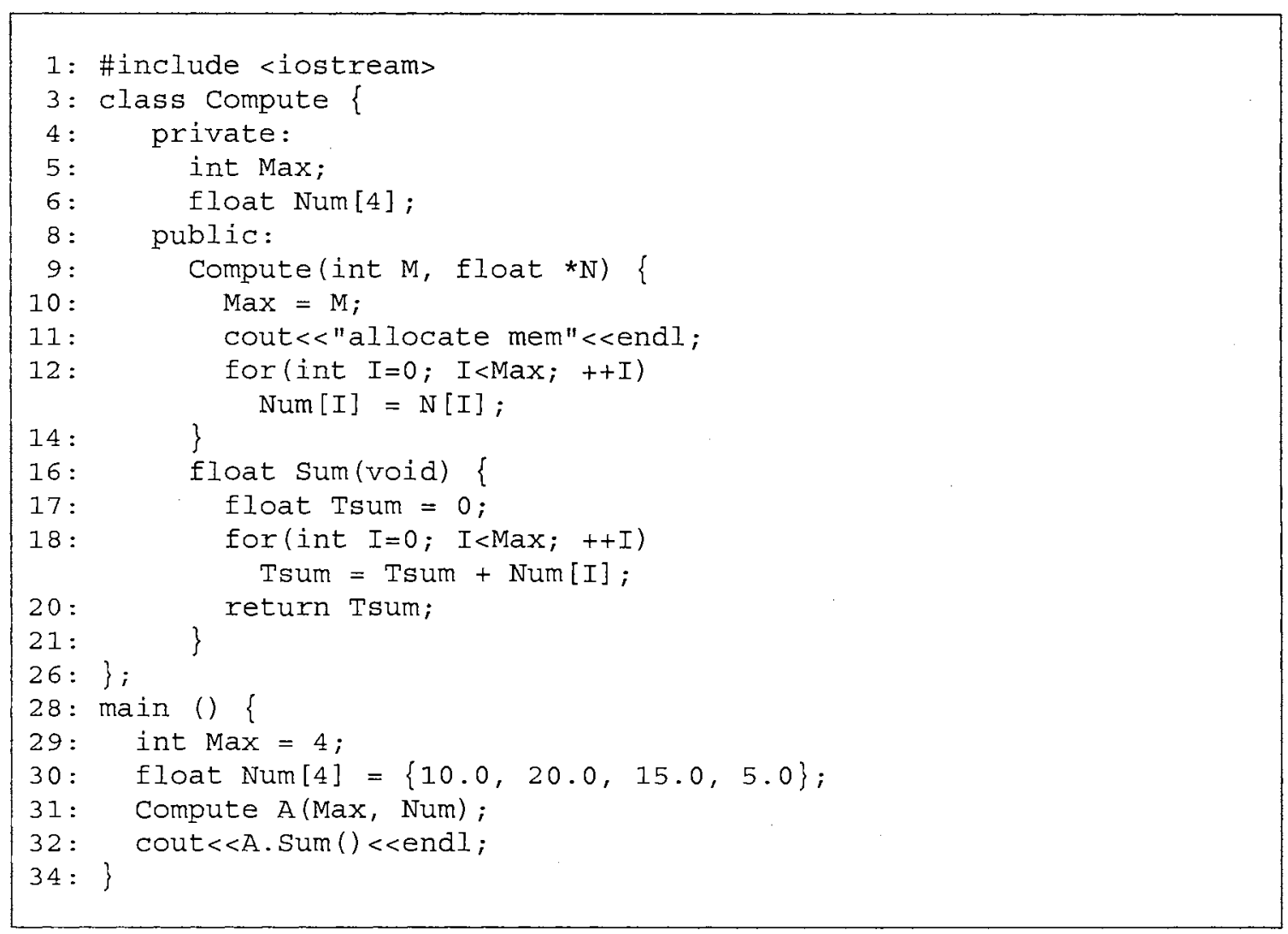

Figure 53. A dynamic program slice computed based on variable sum in line 32 of the program in Figure 44 


\subsection{Problems and Situations in C++ That \\ Were Taken into Account in the Design}

There are eight major problems and situations in $\mathrm{C}++$ that were taken into account in the design of $\mathrm{C}++$ Debug. They are discussed bellow.

1. Problems and situations with classes and objects such as classes, structures, unions, anonymous unions, friend functions, friend classes, inline functions, defining inline functions within a class, parameterized constructors, static class members, static data members, static member functions, the scope resolution operator, nested classes, local classes, passing objects to functions, returning objects, and object assignment.

2. Problems and situations with arrays, pointers, references, and the dynamic allocation operators such as arrays of objects, uninitialized arrays, pointers to objects, type checking $\mathrm{C}++$ pointers, the this pointer, pointers to derived types, pointers to class members, reference parameters, passing references to objects, returning references, independent references, references to derived types, restrictions to references, dynamic allocation operators (i.e., the new operator in $\mathrm{C}++$ ), initializing allocated memory, allocating arrays, allocating objects, the nothrow alternative, and the placement forms of new and delete.

3. Problems and situations with function overloading, copy constructors, and default arguments such as function overloading, overloading constructor functions, overloading a constructor to gain flexibility, initialized and uninitialized objects, copy constructors, finding the address of an overloaded function, the overload anachronism, default function arguments, default arguments vs. overloading, using default arguments correctly, and function overloading and ambiguity. 
4. Problems and situations with operator overloading such as operator overloading using a friend function, using a friend to overload ++ or --, friend operator functions adding flexibility, overloading new and delete, overloading new and delete for arrays, overloading the nothrow version of new and delete, overloading some special operators, overloading [], overloading ( ), overloading $->$, and overloading the comma operator.

5. Problems and situations with inheritance such as base-class access control, inheritance and protected members, protected base-class inheritance, inheriting multiple base classes, constructors, destructors, inheritance, passing parameters to base-class constructors, granting access, and virtual base classes.

6. Problems and situations with virtual functions and polymorphism such as virtual functions, calling a virtual function through a base class reference, the inherited virtual attribute, hierarchical virtual functions, pure virtual functions abstract classes, and late binding.

7. Problems and situations with templates such as generic functions, a function with two generic types, explicitly overloading a generic function, overloading a function template, using standard parameters with template functions, generic function restrictions, applying generic functions, a generic sort, compacting an array, generic classes, a generic array class, using non-type arguments with generic classes, using default arguments with template classes, explicit class specializations, and the typename and export keywords.

8. Problems and situations with exception handling such as exception handling fundamentals, catching class types, using multiple catch statements, handling derived- 
class exceptions, exception handling captions, catching all exceptions, restricting exceptions, rethrowing an exception, terminate() and unexpected(), the uncaught_exception() function, and the exception and bad_exception classes.

\subsection{Dicing Procedures}

Dicing [Lyle 84] [Nanja 90] is the process of identifying a set of statements likely to contain an error. A dice is determined as follows:

1 Compute the slice (Si) for the incorrectly valued output variable(s), which is a subset of KBI (known to be incorrect).

2 Compute the slice (Sc) for the correctly valued output variables(s), which is a subset of CSF (correct so far).

3 Compute ( $\mathrm{Si}-\mathrm{Sc}$ ), which makes up the dice.

Example 6. Observe that a dynamic program slice in Example 4 is a subset of KBI, while a dynamic program slice in Example 5 is a subset of CSF. Consequently, using the definition of dicing, a dice program can be shown as follows

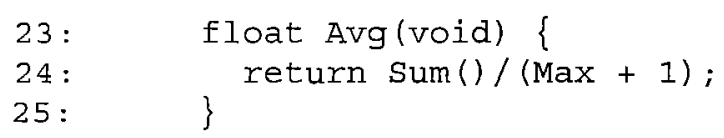

Figure 54. The final program segment after slicing and dicing

Once the procedure is finished, line 24 will be shown as the incorrect line. 


\section{CHAPTER IV}

\section{C++DEBUG}

\subsection{Introduction}

$\mathrm{C}++$ Debug is an interactive debugging tool designed to function as a utility program of the UNIX system. C++Debug was developed based on slicing and dicing techniques. In order for $\mathrm{C}++$ Debug to be more powerful, dynamic slicing rather than static slicing was chosen for implementation. $\mathrm{C}++$ Debug was designed in a way to allow ease and convenience on the part of the user. Using $\mathrm{C}++\mathrm{Debug}$, the user can interact directly with the computer in locating errors in a program. Menus are provided to allow the user to select any one of a number of functions (Slice, Dice, Help, etc.) supported by C++Debug.

To produce the $\mathrm{C}++$ Debug tool, three activities of a software process are introduced: software specification, software development, and software validation. Some parts of the waterfall approach are used to take those three activities and represent them as separate process phases: requirements specifications, software design, implementation, testing, and valuation. In order to make $\mathrm{C}++$ Debug a good piece of software, essential attributes such as maintainability, dependability, efficiency, and usability were considered. 


\subsection{Software specification}

According to Sommerville [Sommerville 01], the intention of this phase is to establish what services are required from $\mathrm{C}++$ Debug and the constraints on $\mathrm{C}++$ Debug's operation and development. The requirements document of $\mathrm{C}++$ Debug is shown in Appendix D.

\subsection{Software Design and Implementation}

In order to convert the $\mathrm{C}++$ Debug software specification, mentioned above in Section 4.2, into an executable system, architectural design, abstract specification, interface design, component design, datastructure design, and algorithm design were carried out [Sommerville 01]. However, because of the limitation of the size of this dissertation, only a few parts are introduced in the following subsections.

\subsection{1 $\mathrm{C}++$ Debug Block Diagram}

C++Debug is comprised of four parts: Cpptrace, Database, Slicer, and Dicer (as shown in Figure 55).

1. Cpptrace was designed as a tool allowing one to follow the execution of a $\mathrm{C}++$ program, statement-by-statement. Cpptrace reads the $\mathrm{C}++$ source program in a file, inserts statements to print the text of each executable statement and the values of all variables referenced or modified, and writes the modified program to generate two major

parts: 1. a trajectory of the program and 2. some databases, where a trajectory is a feasible path that has actually been executed for some input and the databases are a list of reserved words, a list of basic types, identifier information, types, symbol tables, and 


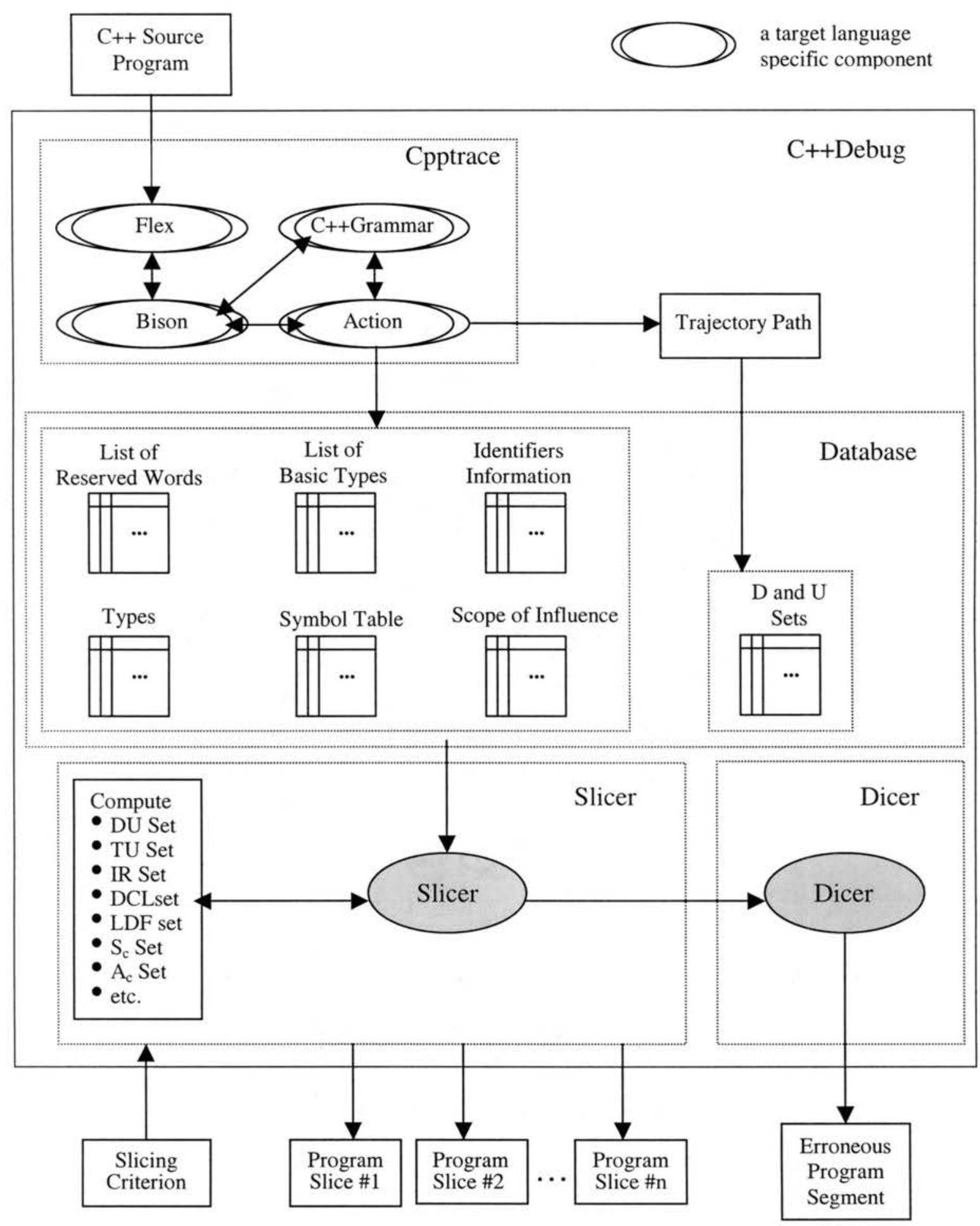

Figure 55. Block diagram of C++Debug

scope of influence. flex and bison are tools used to implement Cpptrace. flex reads 
a specification file containing regular expressions for pattern matching and generates a $\mathrm{C}$ or $\mathrm{C}++$ routine that performs lexical analysis [Flex 01$]$. This routine reads a stream of characters and matches sequences that identify tokens. Bison reads a specification file that codifies the grammar of a language and generates a parsing routine [Bison 00]. This routine groups tokens into meaningful sequences and invokes action routines to act upon them. C++ grammar from Stroustrup's textbook was used in this implementation [Stroustrup 97].

2. Database stores ordered sets of data such as a list of reserved words, a list of basic types, identifier information, types, symbol tables, and scope of influence, etc. All data are created by Cpptrace as a database. The D and U ordered sets of data are computed from the trajectory path. This database is used by Slicer to compute a program slice(s).

3. Slicer was created by using the algorithms in Figure 37. The number of program slices is dependent on the slicing criterion.

4. Dicer was created by using the techniques mentioned in Section 3.6.

\subsubsection{Datastructures}

The datastructures of a source program, functions, a trajectory, sets such as $D, U$ DU, DCL, etc. were implemented based on datastructures shown in Figure 38.

\subsubsection{Symbol Tables}

Symbol tables were designed by following the concepts of symbol tables that are used in cool, the Classroom Object-Oriented Language [Cool 94]. cool is a small language designed for use in an undergraduate compiler course project at the University of California at Berkley [Cool 94]. The key is two functions: enterscope() and 
exitscope(). Function enterscope() makes the table point to a new scope whose parent is the scope it pointed to previously, while function exitscope () makes the table point to the parent scope.

\subsection{Testing and Evaluation}

\subsubsection{Introduction}

After $\mathrm{C}++$ Debug was implemented, the testing process was applied to verify that each unit met its specification (unit testing) and to ensure that the software requirements had been met (integration and system testing) [Sommerville 01]. Testing is the primary means for showing that the implementation has the requisite functionality and other nonfunctional properties [McDermid 93].

\subsubsection{Testing}

Each problem and situation in Section 4.2 was tested independently upon completion of the tool. $\mathrm{C}++$ Debug was also tested on non-trivial programs containing several problems and situations identified. For more information see Appendix E.

\subsubsection{Evaluation}

$\mathrm{C}++$ Debug was evaluated by a number of graduate students at the Computer Science of Oklahoma State University. They used C++Debug to locate errors in their programs. For more information see Appendix E.

\subsection{Limitations}

$\mathrm{C}++$ Debug has some limitations as listed bellow. 
1. Limitation of OS : UNIX

2. Limitation of language: GNU G++

3. Limitations of algorithm: worst-case $O\left(N^{2} \mathrm{~V}\right)$, average-case $\mathrm{O}(\mathrm{N} \log \mathrm{N})$, best-case $\mathrm{O}(\mathrm{N})$, where $\mathrm{N}$ is the \#LOC of the trajectory part, and $\mathrm{V}$ is the maximum number of variables in each line in a debugged program.

4. In the current implementation, limitation of \#LOC of the executable part: 1,000.

\subsection{Program Documentation}

The main purpose of program documentation is to communicate with other people about a finished program [Hedrick 75]. In this study, program documentation for C++Debug was prepared in two parts. The first part involves comments internal to the program. The second part is an auxiliary paper accompanying the program that is included in Chapter III on Software Design. Furthermore, a user's manual was prepared for the convenience of the users of $\mathrm{C}++$ Debug.

\subsection{System Evolution}

System evolution describes the system base, anticipated change due to hardware and software evolution, and the changing user needs [Sommerville 01].

1. System Base

$\mathrm{C}++$ Debug is a slicing and dicing based debugging tool for $\mathrm{C}++$ which runs under UNIX on the SUN machine in the Computer Science Department at OSU.

2. Anticipated Change Due to Hardware Evolution 
$\mathrm{C}++$ Debug is designed to be a portable tool. It is a machine independent tool. It can run on every hardware with a UNIX run-time support. However, C++Debug should be provided on PC as well.

\section{Anticipated Change Due to Software Evolution}

In case of ANSI C++ is updated e.g., if new functions or instructions are added, C++Debug must be updated too.

\section{Changing User Needs}

$\mathrm{C}++$ Debug was designed by using menus in a way to allow ease and convenience on the part of the user. $\mathrm{C}++$ Debug should be provided in a windowing environment as well.

\subsection{Slicing-Based Metrics}

Program slicing is applied to two main areas [Weiser 81]. First, program slicing is used for debugging and maintenance purposes. This is due to the fact that the size of a resulting slice is relatively smaller than the original program in general, thus making it easier to locate errors or to modify the program at the stage of program maintenance. Second, program slicing is used to obtain slicing based program metrics. It allows the analysis of the structure of the program. Weiser proposed three slicing-based program metrics.

i. Coverage compares the length of slices to the length of the entire program. Coverage might be expressed as the ratio of mean slice length to program length. A low coverage value, indicating a long program with many short slices, may indicate a program which has several distinct conceptual purposes.

ii. Overlap is a measure of how many statements in a slice are found only in that slice. This could be computed as the mean 
of the ratios of non-unique to unique statements in each slice. A high overlap might indicate very interdependent code.

iii. Clustering reveals the degree to which slices are reflected in the original code layout. It could be expressed as the mean of the ratio of statements formerly adjacent to total statements in each slice. A low cluster value indicates slices intertwined like spaghetti, while a high cluster value indicates slices physically reflected in the code by statement grouping.

In order to compare the output obtained using $\mathrm{C}++$ Debug (which is dynamic slicing based) with the output obtained using C-Sdicer (which is static slicing based), the test programs must be the same ones as used in Nanja's study in testing C-Sdicer [Nanja 90]. These test programs are listed in Appendix F. The number of output variables and the size of each program is shown in Table I.

The results obtained from C-Sdicer and C++Debug are shown in Tables II and III, respectively.

TABLE I

DESCRIPTION OF THE FIVE TEST PROGRAMS

\begin{tabular}{|c|c|c|c|c|c|}
\hline Metric & P1 & P2 & P3 & P4 & P5 \\
\hline Size (\# of lines) & 120 & 35 & 56 & 67 & 58 \\
\hline $\begin{array}{c}\text { \# of output } \\
\text { variables }\end{array}$ & 26 & 3 & 3 & 10 & 1 \\
\hline
\end{tabular}

(Source: [Nanja 90]) 
TABLE II

SLICING-BASED METRICS OBTAINED FROM C-SDICER FOR THE FIVE TEST PROGRAMS

\begin{tabular}{|c|c|c|c|c|c|}
\hline Metric & P1 & P2 & P3 & P4 & P5 \\
\hline Coverage & 0.86 & 0.77 & 0.57 & 0.75 & 0.83 \\
\hline Overlap & 0 & 4.42 & 10.13 & 0 & 1.00 \\
\hline Clustering & 0.66 & 0.64 & 0.87 & 0.65 & 0.95 \\
\hline
\end{tabular}

(Source: [Nanja 90])

TABLE III

SLICING-BASED METRICS OBTAINED FROM C++DEBUG FOR THE FIVE TEST PROGRAMS

\begin{tabular}{|c|c|c|c|c|c|}
\hline Metric & P1 & P2 & P3 & P4 & P5 \\
\hline Coverage & 0.26 & 0.48 & 0.58 & 0.35 & 0.70 \\
\hline Overlap & 52.33 & 3.60 & 14.60 & 57.00 & 1.00 \\
\hline Clustering & 0.06 & 0.44 & 0.30 & 0.11 & 0.42 \\
\hline
\end{tabular}




\section{CHAPTER V}

\section{SUMMARY, CONCLUSIONS, AND FUTURE WORK}

\subsection{Summary}

Chapter I discusses the necessity of using debugging tools in locating and correcting the errors contained in programs. Included in this chapter are the purposes of the study as well as the organization of the study.

Chapter II describes the general knowledge on program slicing and dicing techniques. The chapter concludes with a discussion of both advantages and disadvantages of dynamic slicing and static slicing, and the procedures used to locate errors in a program using dynamic slicing and dicing techniques.

Chapter III presents the definitions, the algorithms, and the approaches used to compute a program slice and a program segment after dicing. Some examples were shown as well.

Chapter IV presents the steps involved in producing the $\mathrm{C}++$ Debug tool. The C++Debug block diagram, the results of the experiment, slicing-based metrics, testing and evaluation, documents, and the advantages and limitations of $\mathrm{C}++$ Debug were presents also. 


\subsection{Conclusions}

$\mathrm{C}++$ Debug was designed to allow ease and convenience on the part of the user. Using $\mathrm{C}++$ Debug, a user can interact directly with the computer in locating errors in a certain program. For convenience, the program provides menus to allow the user to select any one of the functions contained therein. Based on the results of the experimentation, $\mathrm{C}++$ Debug could generate a new slicing program that is of smaller size than the original source program. The new slicing program still preserves part of the program's original behavior for a specific input. In addition, $\mathrm{C}++$ Debug can be used as a tool like ctrace under UNIX. C++Debug can work on both $\mathrm{C}$ and $\mathrm{C}++$.

By using the $-\mathrm{g}$ option, $\mathrm{C}++$ Debug supports the generation of grammar derivation trees. A users can study how the parser checks the syntax of a program. By using the $-i$ option, all information about $\mathrm{C}++$ Debug can be displayed. One who is interesting in the dynamic slicing area can use the information provided by $\mathrm{C}++$ Debug, such as $\mathrm{D}, \mathrm{U}, \mathrm{DU}$, symbol tables, etc., to investigate the process of slicing, dicing, or compiling in general.

\subsection{Future Work}

Based on the initial experiments with $\mathrm{C}++$ Debug, we found that improvements and additions can be made to $\mathrm{C}++$ Debug in the following aspects.

\subsubsection{Improvements}

The size of $\mathrm{C}++$ Debug after compiling by an optimized compiler is $2,088,720$ bytes. It appears that it should be smaller if some algorithms and memory uses are 
managed better. Time and space complexities are dependent on the size of the trajectory (and not necessarily the size of the source code). To avoid running out of disk space (which is needed to store the trajectory path), the user must know how far the trajectory must go and how much disks space is required. It would be better if $\mathrm{C}++$ Debug can automatically check and tell the user about the sufficiency of the disk space. And it should also estimate the time that $\mathrm{C}++$ Debug is going to take to obtain the slices and the dices.

\subsection{2 $\underline{\text { Additions }}$}

Instead of just menus, some windows should be supported so that a user can view the source code, the trajectory path, the program slice, etc. on the screen. Using a mouse can help a user probably better than using the keyboard in selecting which function to use, or selecting the variables and positions required to compute a slice.

\subsubsection{Future Work}

For a tested $\mathrm{C}++$ program that has pointers, global variables, and static declarations in classes, the algorithm that was used to implement $\mathrm{C}++$ Debug yields an output slice larger than it should be (however, it still gives the correct output and its size is smaller than the original source program). Some lines that should be eliminated are not eliminated. If a better algorithm to manage pointers, global variables, and static declarations in classes is implemented, the size of the resulting slice will be smaller.

It will be desirable if $\mathrm{C}++$ Debug can be made a multi-user-tool. However, in the current implementation, since $\mathrm{C}++$ Debug saves specific files in a local directory, it cannot be used in the multi-user mode. 
Because of the complexities of the $\mathrm{C}++$ symbol table and the time constraint, the current version of $\mathrm{C}++$ Debug cannot treat array elements and fields in dynamic records as individual variables. 


\section{REFERENCES}

[Bison 00] "Bison 1.35 Manual," http://www.gnu.org/manual/bison-1.35/html mono/ bison.html.gz, Last Update: March 2000, Last Access: April 30, 2003.

[Cool 94] "CoolAid: The Cool Reference Manual," http://www.cs.berkeley.edu/ حaiken/ftp/cool-manual.ps, Last Update: January 1994, Last Access: April 30, 2003.

[Flex 01] "Flex, version 2.5 A Fast Scanner Generator Edition 2.5, March 1995," http://www.gnu.org/manual/flex-2.5.4/html mono/flex.html, Last Update: February 23, 2001, Last Access: April 30, 2003.

[Gallagher 90] Keith Brian Gallagher, Using Program Slicing in Software Maintenance, Ph.D. Dissertation, Computer Science Department, University of Maryland, Baltimore County, MD, 1990.

[Gallagher and Lyle 91] Keith B. Gallagher and James R. Lyle, "Using Program Slicing in Software Maintenance," IEEE Transactions on Software Engineering, Vol. 17, No. 8, pp. 751-761, August 1991.

[Hedrick 75] G. E. Hedrick, "Program Documentation," Journal of Data Education, Vol. 15, No. 4, pp. 20-21, July 1975.

[Korel 88] Bogdan Korel, "PELAS-Program Error-Locating Assistant System," IEEE Transactions on Software Engineering, Vol. 14, No. 9, pp. 1253-1260, September 1988.

[Korel and Laski 88] Bogdan Korel and Janusz Laski, "Dynamic Program Slicing," Information Processing Letters, Vol. 29, No. 3, pp. 155-163, October 1988.

[Korel and Laski 90] Bogdan Korel and Janusz Laski, "Dynamic Slicing of Computer Programs," Journal of Systems and Software, Vol. 13, No. 3, pp. 187-195, November 1990.

[Lyle 84] James R. Lyle, Evaluating Variations on Program Slicing for Debugging, Ph.D. Dissertation, Computer Science Department, University of Maryland, College Park, MD, 1984. 
[McDermid 93] John McDermid, Software Engineer's Reference Book, CRC Press, Inc., Boca Raton, Florida, 1993.

[Nanja 90] Sekaran Nanja, An Interactive Debugging Tool for C Based on Program Slicing and Dicing, Master of Science Thesis, Computer Science Department, Oklahoma State University, Stillwater, OK, May 1990.

[Nanja and Samadzadeh 90] Sekaran Nanja and Mansur H. Samadzadeh, "A Slicing/Dicing-Based Debugger for C," The $8^{\text {th }}$ Annual Pacific Northeast Software Quality Conference, Portland, OR, pp. 204-212, October 1990.

[Pohl 94] Ira Pohl, $C++$ for $C$ Programmers, $2^{\text {nd }}$ Edition, The Benjamin/Cummings publishing Company, Inc., Redwood City, CA, 1994.

[Samadzadeh and Wichaipanitch 93] Mansur H. Samadzadeh and Winai Wichaipanitch, "An Interactive Debugging tool for C based on Dynamic Slicing", Proceedings of the 1993 ACM Computer Science Conference, Indianapolis, IN, pp. 30-37, February 1993.

[Sommerville 01] Ian Sommerville, Software Engineering, $6^{\text {th }}$ Edition, Addison Wesley Publishing Company, New York, N.Y., 2001.

[Stroustrup 97] B. Stroustrup, $C++$ Programming Language, $3^{\text {rd }}$ Edition, AddisonWesley, Inc., Reading, Massachusetts, 1997.

[Tassel 74] Dennie V. Tassel, Program Style, Design, Efficiency, Debugging, and Testing, Prentice-Hall, Inc., Englewood Cliffs, NJ, 1974.

[Weiser 81] Mark Weiser, "Program Slicing," Proceedings of the Fifth International Conference on Software Engineering, San Diego, CA, pp. 439-449, March 1981.

[Weiser 82] Mark Weiser, "Programmers Use Slices When Debugging," Communications of the ACM, Vol. 25, No. 7, pp. 446-452, July 1982.

[Weiser 84] Mark Weiser, "Program Slicing," IEEE Transactions on Software Engineering, Vol. SE-10, No. 4, pp. 352-357, July 1984.

[Weiser and Lyle 86] Mark Weiser and James R. Lyle, "Experiments on Slicing-Based Debugging Aids," a paper presented at The First Workshop on Empirical Studies of Programmers, (Soloway, E. and Iyengar, S., Editors), Ablex Publishing Corporation, Norwood, NJ, pp. 187-197, 1986. 


\section{APPENDICES}




\section{APPENDIX A}

\section{GLOSSARY}

Action: An action, e.g., an instruction $\mathrm{X}$ at position $\mathrm{p}$ in a trajectory $\mathrm{T}$, sometimes represented as the pair $(\mathrm{X}, \mathrm{p})$. For example, $4^{4}$ and $4^{9}$ in trajectory $\mathrm{T}$ in Figure 10 are actions that involve the same instruction 4 . See also trajectory.

$\operatorname{Back}(\mathrm{T}, \mathrm{q})$ : Denotes the sublist $\left\langle\mathrm{X}_{\mathrm{q}+1}, \ldots, \mathrm{X}_{\mathrm{m}}\right\rangle$ of $\mathrm{T}$, consisting of elements that follows $\mathrm{T}(\mathrm{q})$, a trajectory at position $\mathrm{q}$. Where $\mathrm{T}=\left\langle\mathrm{x}_{1}, \mathrm{X}_{2}, \ldots, \mathrm{X}_{\mathrm{m}}\right\rangle$ denotes a trajectory of length $\mathrm{m}$, and $\mathrm{q}$ is a position in $\mathrm{T}, 1<\mathrm{q}<\mathrm{m}$. See also trajectory, $\operatorname{Front}(\mathrm{T}, \mathrm{q})$ and $\operatorname{Del}(\mathrm{T}, \mathrm{R})$.

Bug: $\quad$ An error in a computer program that may be either a syntax error or a logical error.

Called action: An action $\mathrm{X}^{\mathrm{p}}$ is a called action if $\mathrm{X}$ is a called function instruction.

Called-to-Calling: Occurred when a slice is computed from a called action first and then a calling action.

Calling action: An action $\mathrm{x}^{\mathrm{p}}$ is a calling action if $\mathrm{x}$ is a calling function instruction.

Calling-to-Called: Occurred when a slice is computed from a calling action first and then a called action.

$\mathrm{D}\left(\mathrm{X}^{\mathrm{p}}\right)$ : The set of variables that are defined in action $\mathrm{X}^{\mathrm{p}}$. For example, in the execution trace of Figure 10, $18^{15}$ Avg := Sum/(MaxData + 1); Avg is a set of variables that are defined in $18^{15}, \mathrm{D}\left(18^{15}\right)$. See also trajectory, $U\left(\mathrm{X}^{\mathrm{P}}\right)$, $\operatorname{DU}\left(\mathrm{X}^{\mathrm{p}}\right), \operatorname{IR}\left(\mathrm{X}^{\mathrm{p}}\right)$, and $\mathrm{TC}\left(\mathrm{X}^{\mathrm{p}}\right)$.

Debugging: A process to locate and correct errors or bugs. Debugging differs from testing in that testing is used to determine whether a program is working properly, whereas debugging localizes and corrects the errors. 
$\operatorname{DEL}(T, R)$ : A subtrajectory obtained from $T$ by deleting from it all elements $T(i)$ that satisfy $\mathrm{R}$. Where $\mathrm{T}=\left\langle\mathrm{X}_{1}, \mathrm{X}_{2}, \ldots, \mathrm{X}_{\mathrm{m}}\right\rangle$ denotes a trajectory of length $\mathrm{m}$, and $\mathrm{q}$ is a position in $T, 1<q<m$. Where $R$ is a predicate on the set of instructions in $T$. See also trajectory, $\operatorname{Front}(T, q)$, and Back $(T, R)$.

$\mathrm{DF}_{\text {name }}\left(\mathrm{X}^{\mathrm{p}}\right)$ : A set of variables that are defined in action $\mathrm{X}^{\mathrm{p}}$; where $\mathrm{X}^{\mathrm{p}} \in \mathrm{M}(\mathrm{TF}$ name $)$.

Dicing: The process of identifying a set of statements prone to contain an error.

$\mathrm{DU}\left(\mathrm{x}^{\mathrm{p}}\right)$ : Definition-Use Relation, a binary relation on $\mathrm{M}(\mathrm{T})$ in which one action assigns a value to an item of data and the other action uses that value. For example, in the execution trace of Figure $10,2^{2}$ assigns a value to variable Count and $4^{4}, 9^{5}, 16^{7}$, and $17^{8}$ use that value. See also trajectory, $M(T)$, $\mathrm{D}\left(\mathrm{X}^{\mathrm{P}}\right), \mathrm{U}\left(\mathrm{X}^{\mathrm{P}}\right), \operatorname{IR}\left(\mathrm{X}^{\mathrm{P}}\right)$, and $\mathrm{TC}\left(\mathrm{X}^{\mathrm{P}}\right)$.

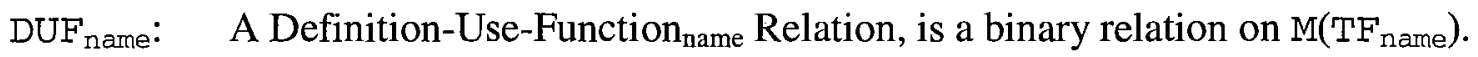

$\mathrm{DV}\left(\mathrm{V}, \mathrm{TF} \mathrm{F}_{\text {name }}\right)$ : A function that return a set of line numbers, where $\mathrm{V}$ is set of variables.

Dynamic Slicing: A slicing method defined on the basis of a computation rather than all computations. It generates a dynamic program slice by computing from the executable part of the original source program. See also program slicing and static slicing.

EI: $\quad$ A Called-to-Calling Relation between 2 functions, $X^{p}$ IE $Y^{t}$, iff both are in a Called-to-Calling situation, where $X^{p}$ is a calling action and $Y^{t}$ is a called action.

$F_{\text {name }}$ : A function, a set of instruction X's which is in the scope of influence of function name.

Feasible Path: Let a flowgraph of program $P$ be a directed graph and $C=(N, A, s, e)$ be a slicing criterion where:

1. $\mathrm{N}$ is a set of nodes,

2. $A$ is a binary relation on $\mathrm{N}$ (a subset of $\mathrm{N} \times \mathrm{N}$ ), referred to as a set of arcs,

3. $s \in \mathrm{N}$ is a unique entry node, and

4. $e \in \mathrm{N}$ is a unique exit node.

A node in $\mathrm{N}$ is referred to as an instruction, including a single instruction and a control instruction. A single instruction includes, for example, an assignment statement, an input or output statement, etc. A control instruction includes such statements as if-then-else or while statements, which are called test instructions. An $\operatorname{arc}(n, m) \in A$ corresponds to a 
possible transfer of control from instruction $n$ to instruction $m$. A path from entry node $s$ to some node $k$ when $k \in N$ is called a sequence $<n_{1}, n_{2}, \ldots$, $n_{\mathrm{q}}>$ of instructions, such that $\mathrm{n}_{1}=\mathrm{s}, \mathrm{n}_{\mathrm{q}}=\mathrm{k}$ and $\left(\mathrm{n}_{\mathrm{i}}, \mathrm{n}_{\mathrm{i}+1}\right) \in \mathrm{A}$, for all $\mathrm{n}_{\mathrm{i}}, 1$ $<i<$ q. If there is input data, which causes the path to be traversed during program execution, the path is called feasible.

FN(q): $\quad$ A function name such that $X^{q}, X$ is in the scope of influence.

Front $(\mathrm{T}, \mathrm{q})$ : The sublist $\left\langle\mathrm{X}_{1}, \mathrm{X}_{2}, \ldots, \mathrm{X}_{\mathrm{q}}>\right.$ of $\mathrm{T}$, consisting of the first $\mathrm{q}$ elements of $\mathrm{T}$, where $T=\left\langle X_{1}, X_{2}, \ldots, X_{m}\right\rangle$ denotes a trajectory of length $m$, and $q$ is a position in $T, 1<q<m$. See also trajectory, $\operatorname{Back}(T, q)$, and $D E L(T, R)$.

$G(X): \quad$ A set of variables and precedences that are declared as a global declaration.

IE: A Calling-to-Called Relation between 2 functions, $X^{p}$ IE $Y^{t}$, iff both are in a Calling-to-Called situation, where $\mathrm{X}^{\mathrm{p}}$ is a called action and $\mathrm{Y}^{\mathrm{t}}$ is a calling action.

IR $\left(X^{p}\right)$ : Let $X^{\mathfrak{p}}$ IR $Y^{t}$, iff $X=Y$ is the Identity Relation IR on M(Front $\left.(T, q)\right)$. See also trajectory, $\mathrm{M}(\mathrm{T}), \mathrm{D}\left(\mathrm{X}^{\mathrm{p}}\right), \mathrm{U}\left(\mathrm{X}^{\mathrm{p}}\right), \mathrm{DU}\left(\mathrm{X}^{\mathrm{p}}\right), \mathrm{TC}\left(\mathrm{X}^{\mathrm{p}}\right)$, and $\mathrm{Front}(\mathrm{T}, \mathrm{q})$.

$I F_{\text {name }}: \quad$ An Identity Relation in Function ${ }_{\text {name }}, X^{p} I R F_{\text {name }} Y^{\mathrm{t}}$, iff $X=Y$ is the identity relation $I_{R F_{\text {name }}}$ on $\mathrm{M}\left(\mathrm{Front}\left(\mathrm{TF}_{\text {name }}, \mathrm{q}\right)\right)$.

Last Definition: Last definition $\mathrm{X}^{\mathrm{p}}$ of variable $\mathrm{v}$ at $\mathrm{t}$ is the action which has last assigned a value to $\mathrm{v}$ when $\mathrm{t}$ is reached on trajectory $\mathrm{T}$. See also trajectory.

$\mathrm{LF}_{\text {name }}\left(\mathrm{X}^{\mathrm{p}}\right)$ : A set of variables and $\mathrm{C}++$ precedences that are declared as a local declaration in function name.

$M(T)$ : $\quad$ A set of actions in a given trajectory $T$, where $M(T)=\{(X, p): T(p)=X\}$. See also trajectory.

$M\left(T F_{\text {name }}\right)$ : A set of actions in a given function of a given trajectory $T F_{\text {name, }}$ where $M\left(T_{\text {name }}\right)=\left\{X^{\mathrm{p}}\right.$ : instruction $X$ at position $\mathrm{p}$ in trajectory $\left.\mathrm{TF}_{\text {name }}\right\}$. $\mathrm{M}\left(\mathrm{TF}_{\text {name }}\right)$ is a subset of $\mathrm{M}(\mathrm{T})$.

P: $\quad$ A set of instruction X's, in a C++ tested program.

Preprocessor: A separate first step in compilation, e.g., \#include, \#define, or \#if.

Program Slicing: A segment of a program that is separated and identified based on the premise that instead of localizing errors in the original program, which can be of large size, one can locate such errors in a program of smaller size which is sliced from the original program but still preserves part of the 
original program's behavior for a particular input or relative to a particular variable.

Slicing Criterion: The specification that a behavior of interest of a program can be expressed as the values of some set of the variables at some set of statements.

Static Slicing: A method defined on the basis of all computations and used for generating a static program slices. The computations of static slices are done directly from the original source program. See also program slicing and dynamic slicing.

$T(p)$ : The abstract list of a trajectory $T$ whose elements are accessed at position $p$, e.g., for $\mathrm{T}$ in Figure 10, $\mathrm{T}(3)=3, \mathrm{~T}(5)=9$, etc. See also trajectory.

$\mathrm{TC}\left(\mathrm{X}^{\mathrm{p}}\right)$ : Test-Control Relation, a binary relation on $\mathrm{M}(\mathrm{T})$, capturing the effect between test actions and actions that have been chosen to execute by those test actions. For example, in the execution trace of Figure 10, the scope of the test action $\quad 4^{4}$ influences the execution of $9^{5}, 10^{6}, 16^{7}$, and $17^{8}$, but it does not influence the execution of $13^{10}, 14^{11}, 16^{12}$, and $17^{13}$. See also trajectory, $M(T), D\left(X^{P}\right), U\left(X^{P}\right), D U\left(X^{P}\right)$, and $I R\left(X^{P}\right)$.

$\mathrm{TCF}_{\text {name: }} \quad \mathrm{A}$ Test-Control-Function ${ }_{\text {name }}$ Relation, is a binary relation on $\mathrm{M}\left(\mathrm{TF}_{\text {name }}\right)$.

Test Action: An action $\mathrm{X}^{\mathrm{p}}$ is a test action if $\mathrm{X}$ is a test instruction. See also trajectory.

Test Instruction Statements: A control instruction such as an if-then-else or a while statement.

$\mathrm{TF}_{\text {name: }} \quad$ A function trajectory, a feasible path of a function name that has actually been executed for some input. $T F_{\text {name }}$ is a sublist of $\mathrm{T}$.

Trajectory: A feasible path that has actually been executed for some input. For example, $\quad<1,2,3,4,9,10,16,17,4,13,14,16,17,4,18,19>$ is the trajectory when the program in Figure 1 is executed on the input data MaxData $=2$, Data $=(3,5)$. A trajectory will be illustrated in terms of a pair (instruction, its position in the trajectory) rather than the instruction itself so as to distinguish between multiple occurrences of the same instruction in the trajectory. For example, instruction $\mathrm{X}$ at position $\mathrm{p}$ in $\mathrm{T}$ will be represented by the pair $(X, p)$. For ease of understanding, the pair $(X, p)$ will be replaced by $X^{p}$ and will be referred to as an action. For example, $4^{4}$ and $4^{9}$ in trajectory $\mathrm{T}$ in Figure 10 are actions that involves the same instruction 4. See also feasible path. 
$\mathrm{U}\left(\mathrm{X}^{\mathrm{p}}\right)$ : $\quad$ The set of variables that are used in $\mathrm{X}^{\mathrm{p}}$. For example, in the execution trace of Figure 10, $18^{15}$ Avg := Sum/(MaxData +1$)$; Sum and MaxData are a set of variables that are used in $18^{15}, \mathrm{U}\left(18^{15}\right)$. See also trajectory, $\mathrm{D}\left(\mathrm{X}^{\mathrm{P}}\right)$, $\operatorname{DU}\left(\mathrm{X}^{\mathrm{p}}\right), \operatorname{IR}\left(\mathrm{X}^{\mathrm{p}}\right)$, and $\mathrm{TC}\left(\mathrm{X}^{\mathrm{p}}\right)$.

$\mathrm{UF}_{\text {name }}\left(\mathrm{X}^{\mathrm{p}}\right)$ : A set of variables that are used in action $\mathrm{X}^{\mathrm{p}}$; where $\mathrm{X}^{\mathrm{p}} \in \mathrm{M}(\mathrm{TF}$ name $)$.

VDU(FunctionName): A set of variables that are used, $\mathrm{UF}_{\text {name, }}$, and defined, $\mathrm{DF}_{\text {name, }}$, in the given function name.

$\operatorname{VS}\left(\mathrm{X}_{\mathrm{DU}}\right): \quad$ A variable scope relation at $\mathrm{X}_{\mathrm{DU}}$, be a set of instructions $\mathrm{X}_{\mathrm{DCL}}$, where $\mathrm{X}_{\mathrm{DU}}$ is in the scope of influence of $\mathrm{X}_{\mathrm{DCL}}$.

$\mathrm{X}: \quad$ An instruction in a program and $\mathrm{X} \in \mathrm{IN}^{+}$. See also program $\mathrm{P}$.

$\mathrm{X}_{\mathrm{DCL}}$ : $\quad$ An instruction that declared variables such as "int $I_{\text {; }}$ ”.

$\mathrm{X}_{\mathrm{DU}}$ : $\quad$ An instruction that used or defined the variables that declared by $\mathrm{X}_{\mathrm{DCL}}$, where variables that used or defined are in the scope of influence of variables that declared in $\mathrm{X}_{\mathrm{DCL}}$.

$\mathrm{X}^{\mathrm{p}}$ : $\quad$ An action, e.g., an instruction $\mathrm{X}$ at position $\mathrm{p}$ in a trajectory $\mathrm{T}$, sometimes represented as the pair $(\mathrm{X}, \mathrm{p})$. For example, $4^{4}$ and $4^{9}$ in trajectory $\mathrm{T}$ in Figure 10 are actions that involve the same instruction 4. See also trajectory.

$\mathrm{Y}^{\mathrm{t}}$ An action, instruction $\mathrm{Y}$ at position $\mathrm{t}$ in a trajectory $\mathrm{T}$. See also $\mathrm{X}^{\mathrm{P}}$. 


\title{
APPENDIX B \\ USER'S MANUAL FOR C++DEBUG
}

\author{
B.1 Introduction
}

$\mathrm{C}++$ Debug is a slicing and dicing based debugging tool for ANSI $\mathrm{C}++$ that runs under the UNIX or Linux operating system. It has been designed in a way to provide ease and convenience to the user. Using $\mathrm{C}++$ Debug, the user can interact with the computer in locating errors in a program. For convenience of the user, the menu shown in Figure 56 allows the user to select any of the available functions.

\section{B.2 C++Debug's Commands}

At a UNIX prompt, C++Debug is invoked by typing the following command: $\$ C++$ Debug [prog_name]

where \$ is a UNIX Bourne shell prompt and prog_name, the optional parameter, is the name of the program to be loaded into the $\mathrm{C}++$ Debug environment. Once this command is executed, $\mathrm{C}++$ Debug will return to the help menu so that additional commands can be executed. 


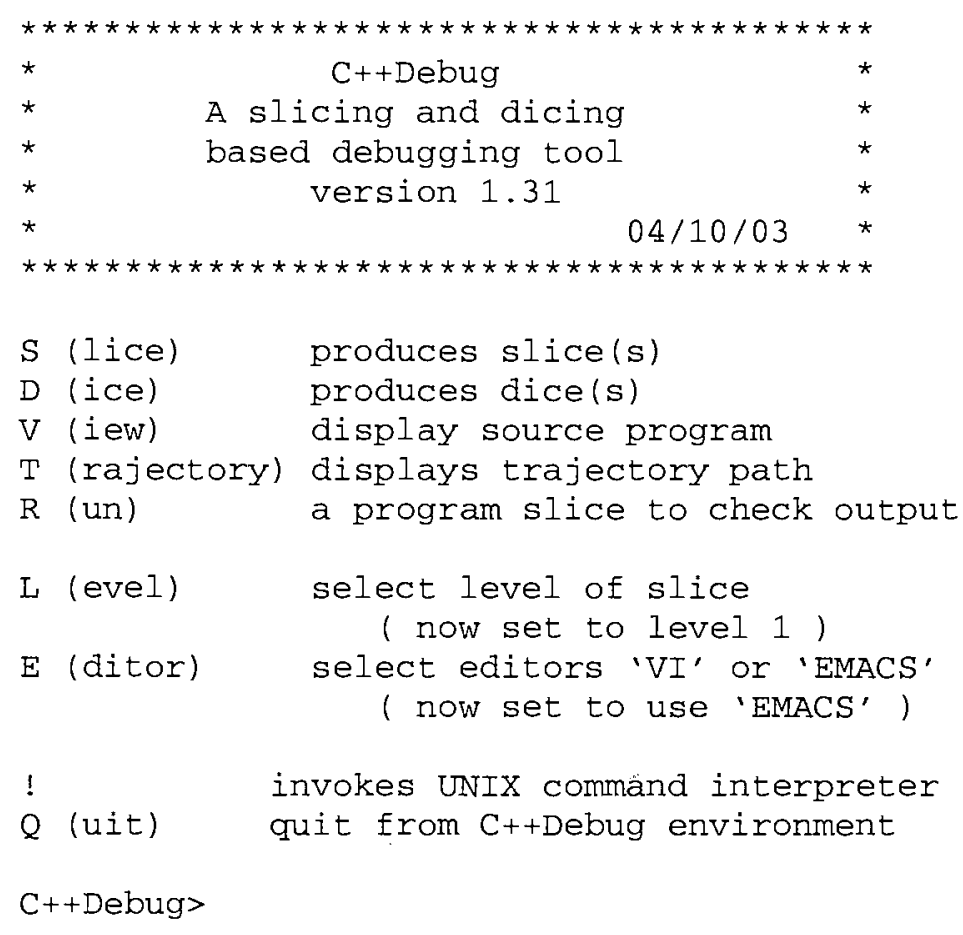

Figure 56. Help menu and prompt

The following commands are available within the $\mathrm{C}++$ Debug environment. They are explained in the order that they appear in the $\mathrm{C}++$ Debug menu.

$\mathrm{S}$ or $\quad$ Produces a slice of the program currently resident within the C++Debug Slice environment with the variables supplied to it as its arguments.

Example:

C++Debug $>S$ line_num var1 [var2...] C++Debug $>$ Slice line_num var1 [var2...]

where Iine_num is a valid line number in the program and var1, var2 are variables in the program. This command requires a line number and at least one variable to produce a slice.

D or Produces a dice, given a set of variables and a line number.

Dice

Example:

$C++$ Debug $>D$ line_num var1 [var2...] / var3 [var4...]

$C++$ Debug $>$ Dice 1 ine num $\operatorname{var} 1[\operatorname{var} 2 \ldots] / \operatorname{var} 3[\operatorname{var} 4 .$.$] ]$ 
where Iine_num is a valid line number in the program and var1, $\operatorname{var2,}$ var3, and var4 are variables in the program. This command requires a line number and at least two variables to be supplied, separated by a $\mid$.

V or Views or displays the source program resident within the C++Debug View environment on the display unit. VI or EMACS is used to view the source program. One can select to uses VI or EMACS by using $E$ or Editor.

T or

Trajectory

Displays the trajectory path or the execution trace of the source program resident within the $\mathrm{C}++$ Debug environment on the display unit.

$\mathrm{R}$ or Compiles and run the slice program currently resident within the Run $\quad \mathrm{C}++$ Debug environment with the $\mathrm{G}++$ compiler (to compare the slice output with the original program output).

L or In level 1:

Level

$\mathrm{C}++$ Debug can work with any size source program. C++Debug will use some harddisk space (about $1 \mathrm{~K}$-byte) to keep track of information. Level 1 yields an output slice larger than level 2.

In level 2:

Level 2 allows the user to get a program slice smaller than Level 1. The limitation is that $\mathrm{C}++$ Debug will use more of your space than Level 1 in order to keep track of the trajectory path.

E or Selects editor from VI, EMACS, or EMACS for windows.

Editor

$! \quad$ Invokes a UNIX command.

Example:

C++Debug $>$ ! Is - 1

C++Debug $>$ ! who

$Q$ or Exits from the C++Debug environment to the UNIX system.

Quit 


\section{B.3 Tutorial}

In order to make it easier to understand, this tutorial walks the user through $\mathrm{C}++$ Debug step by step. The tutorial guide you from the basic commands, such as view manual or version, to the more complicated commands such as Slice or Dice.

\section{B.3.1 A Step-by-Step Guide}

1. At the UNIX prompt, type C++Debug. You will see the following message:

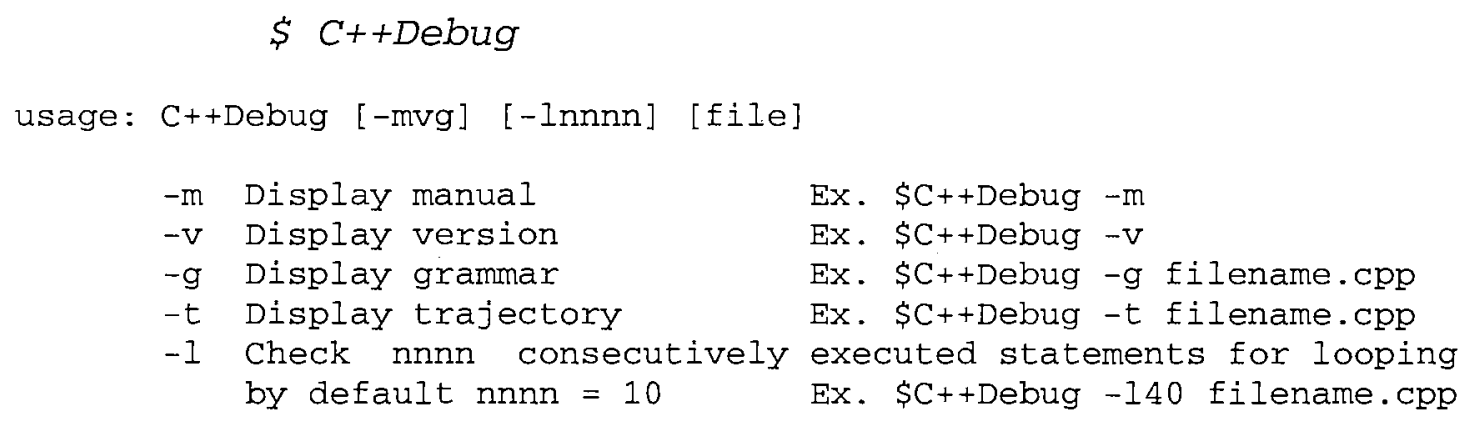

2. Type

$$
\$ C++ \text { Debug }-m
$$

C++Debug's user's manual should be display on the screen.

3. Type

$$
\$ C++ \text { Debug }-V
$$

C++Debug's version should be display on the screen.

4. Type

$$
\$ C++ \text { Debug }-g \text { Test1. } c p p
$$

For a grammar, you will see the tree corresponding to the derivation of program Test1.cpp (see Subsection B.3.2.1). The tree is up side down (the root is at the bottom). 


\begin{tabular}{|l|l|}
\hline \multicolumn{1}{|c|}{ Display on the screen } & \multicolumn{1}{c|}{ Should look like } \\
\hline$\ldots \ldots$ & $\begin{array}{l}\text { Program: } \\
\text { declaration_seq }\end{array}$ \\
$\cdots \ldots$ & $\begin{array}{l}\text { declaration_seq: } \\
\text { declaration_seq declaration } \\
\text { declaration: } \\
\text { function_definition } \\
\text { declaration_seq: } \\
\text { declaration_seq declaration }\end{array}$ \\
$\begin{array}{l}\text { Program: } \\
\text { declaration_seq }\end{array}$ & $\begin{array}{l}\text { declaration: } \\
\text { function_definition }\end{array}$ \\
& \begin{tabular}{l} 
compound_statement \\
\hline
\end{tabular} \\
\hline
\end{tabular}

Or, it can be represented graphically as follow:

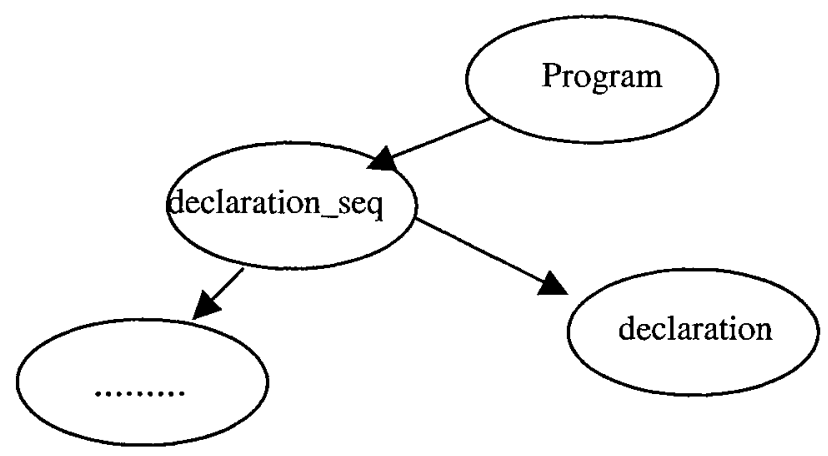

This area is left for someone who is interested and would like to translate a derivation tree from the text mode to graphical representation (a directed graph).

5. Type

$$
\$ C++ \text { Debug }-t \text { Test1. cpp }
$$

The trajectory path that allows you to follow the execution of a $\mathrm{C}++$ program, statement by statement, will show on the screen as follows: 


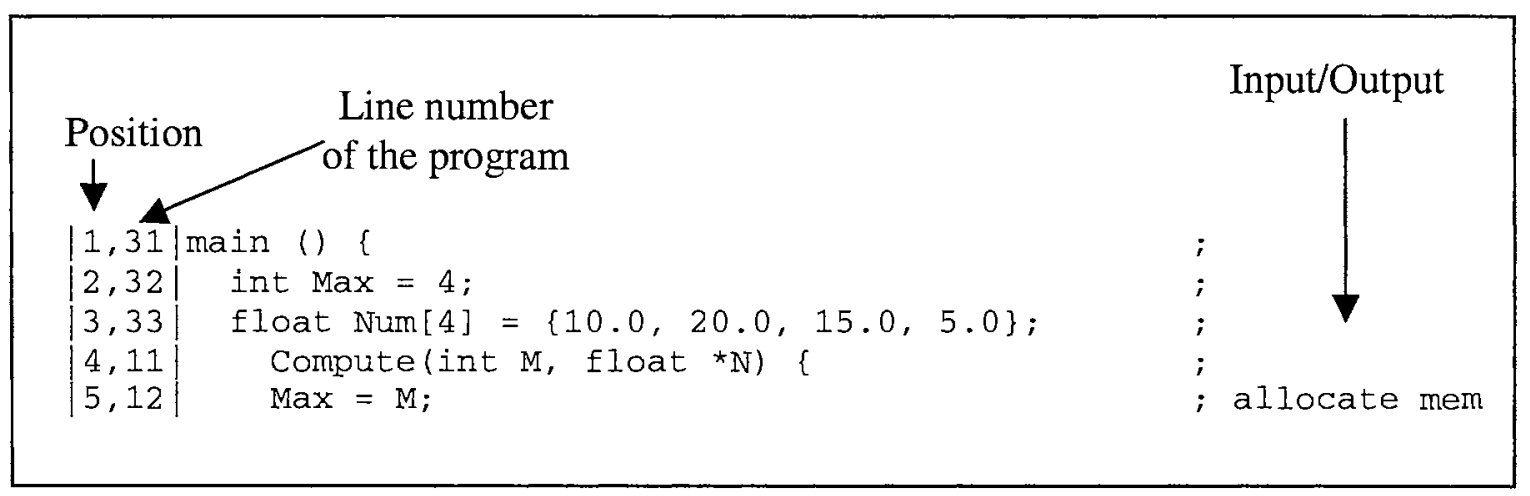

Figure 57. The trajectory path

where $1,2,3, \ldots$ are positions and $31,32,33,11,12, \ldots$ are line numbers of program Test 1 . cpp. The input and/or output of the program will be shown after ';'.

Now you can keep track of your program as to what line numbers are running.

6. Let's try program Test2. cpp (see Subsection B.3.2.2). with multiple loops, type

$$
\$ C++ \text { Debug }-110 \text { Test2.cpp }
$$

Be careful, -110 is letter e11 and one zero.

Compare it with:

$$
\$ C++ \text { Debug }-1100 \text { Test2.cpp }
$$

Now you have some idea about $\mathrm{C}++$ Debug and how it works as a cpptrace tool, and what a trajectory is. However, locating errors in a trajectory still requires work. $\mathrm{C}++$ Debug can help users to find errors. Please follow the examples to gain facility in using the tool.

Next, let's try program slicing.

7. Type

$$
\$ C++ \text { Debug Test } 1 . C p p
$$


The screen should display the menu shown in Figure 56.

\section{Type}

$$
\text { C++Debug> s } 21 \text { Tsum }
$$

That means you are going to slice a program based on variable Tsum at line 21 . The new program (which is smaller than the original) will be shown in the editor mode (by selecting between VI and EMACS).

Using slicing, one obtains a new program of generally smaller size that still maintains all aspects of the original program's behavior with respect to the criterion variable.

Now, let's try another line number:

$$
\text { C++Debug> s } 32 \operatorname{Max}
$$

That means you are going to slice a program based on variable Max at line 32 . The new program (which is smaller than the original) will be shown.

Now, let's try another line number:

$$
C++ \text { Debug> s } 10 \text { public }
$$

You will get an empty slice, because that line has not been executed.

Tip: To select 'Line' and 'Variable', one must be careful. One must make sure that the line will be executed.

For example,

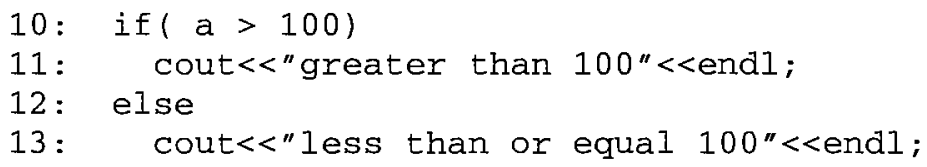

Assume that $\mathrm{a}=150$. 
In this case, suppose that you select line 13 , you will get an empty slice, because line 13 has not been executed.

Let's slice program Test 1 . cpp based on variable Sum and Avg by first typing C++Debug> s 35 Sum

you should get a slice program based on variable sum at line 35, and by typing

$$
\text { C++Debug> s } 36 \text { Avg }
$$

you should get a slice program based on variable Avg at line 36 .

9. Dice

Now you know to slice a program.

Now, let's try 'Dice'.

Dicing technique is used to compare two or more slices resulting from the program slicing technique in order to identify the set of statements that are likely to contain an error.

In the previous examples, Avg gives an incorrect output. The correct output of Avg must be

$$
(10.0+20.0+15.0+5.0) / 4=12.5
$$

However, Sum gives the correct output of 50. Therefore you can locate the error in the program by using the dicing technique.

$$
\text { C++Debug> d } 36 \text { Avg } 35 \text { Sum }
$$

where 36 Avg is a slice at line 36 based on variable Avg (which gave an incorrect result) and 35 Sum is a slice at line 35 based on variable Sum (which gave a correct result).

You should get the following output: 


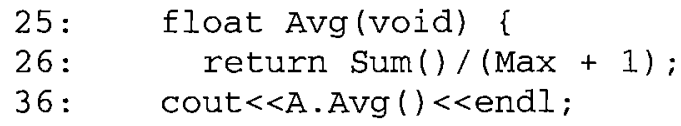

You know that line 25 and line 36 are correct (obviously). So we have line 26 left.

We find that line 26 should be

26: return Sum ()/(Max); // which is correct

instead of

26: return $\operatorname{Sum}() /(\operatorname{Max}+1) ;$

There exists an extra ' +1 ', which is incorrect.

\section{B.3.2 Source Code Listing}

\section{B.3.2.1 Test1.cpp}

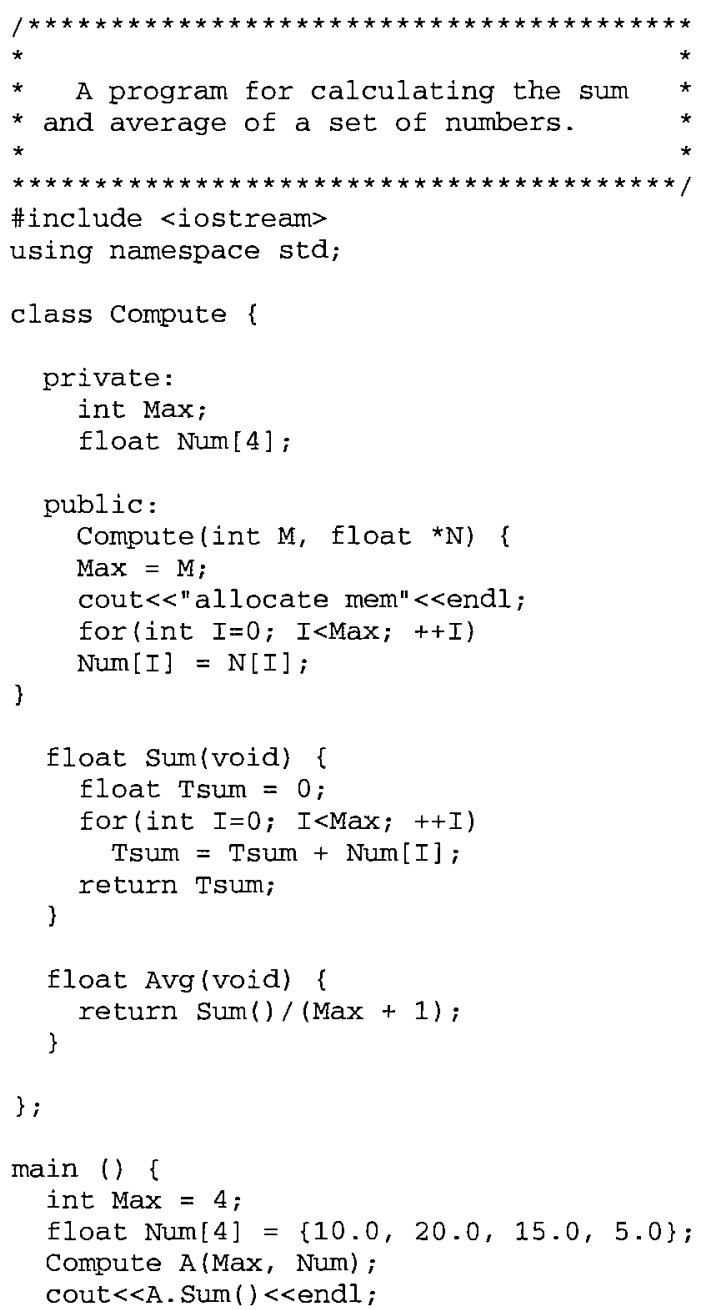


$\operatorname{cou} t<<A . \operatorname{Avg}()<<e n d l$

\section{B.3.2.2 Test2.cpp}

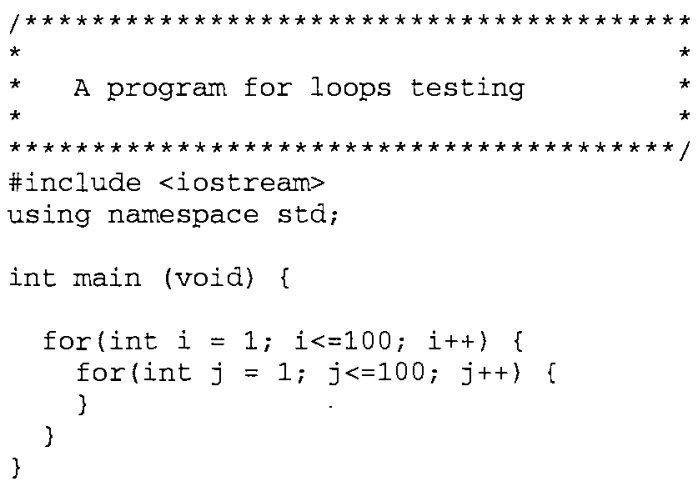




\author{
APPENDIX C \\ DATASTRUCTURE DESIGN FOR C++DBUG \\ BASED ON DYNAMIC PROGRAMSLICING AND DICING
}

\author{
C.1 Introduction
}

In order to design datastructures for $\mathrm{C}++$ Debug, the grammar that appears in $\mathrm{C}++$ Programming Language Third Edition written by Bjarne Stroustrup, the creator of C++, has been used in this design. The design was started from basic functions and expanded to pointers, structures, functions, and classes.

\title{
C.2 Types and Declarations
}

\section{C.2.1 Types}

Every name (identifier) in a $\mathrm{C}++$ program has a type associated with it. The type determines what operations can be applied to the name and how such operations are interpreted. In this design, the data structure of Types used to store all built-in and userdefined types used in the program is shown in Figure 58.

InsertTypes(ID) is used to manage database in Figure 60, which includes all of these six functions:

InsertBasicType(ID) is used to insert basic types into the list such as bool, char, int, double, void, unsigned, long, short, etc.

InsertClas sName(ID) is used to insert class names into the list. 


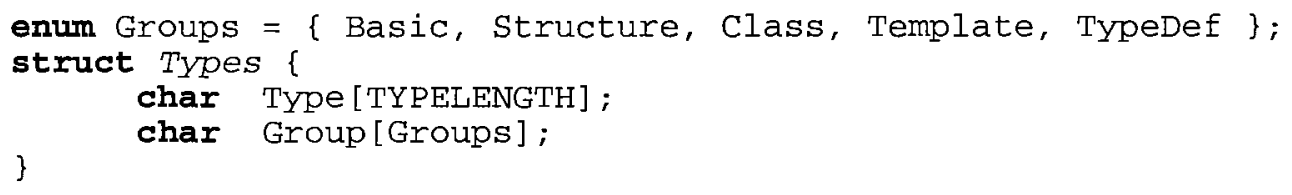

Figure 58. Data structure of Types

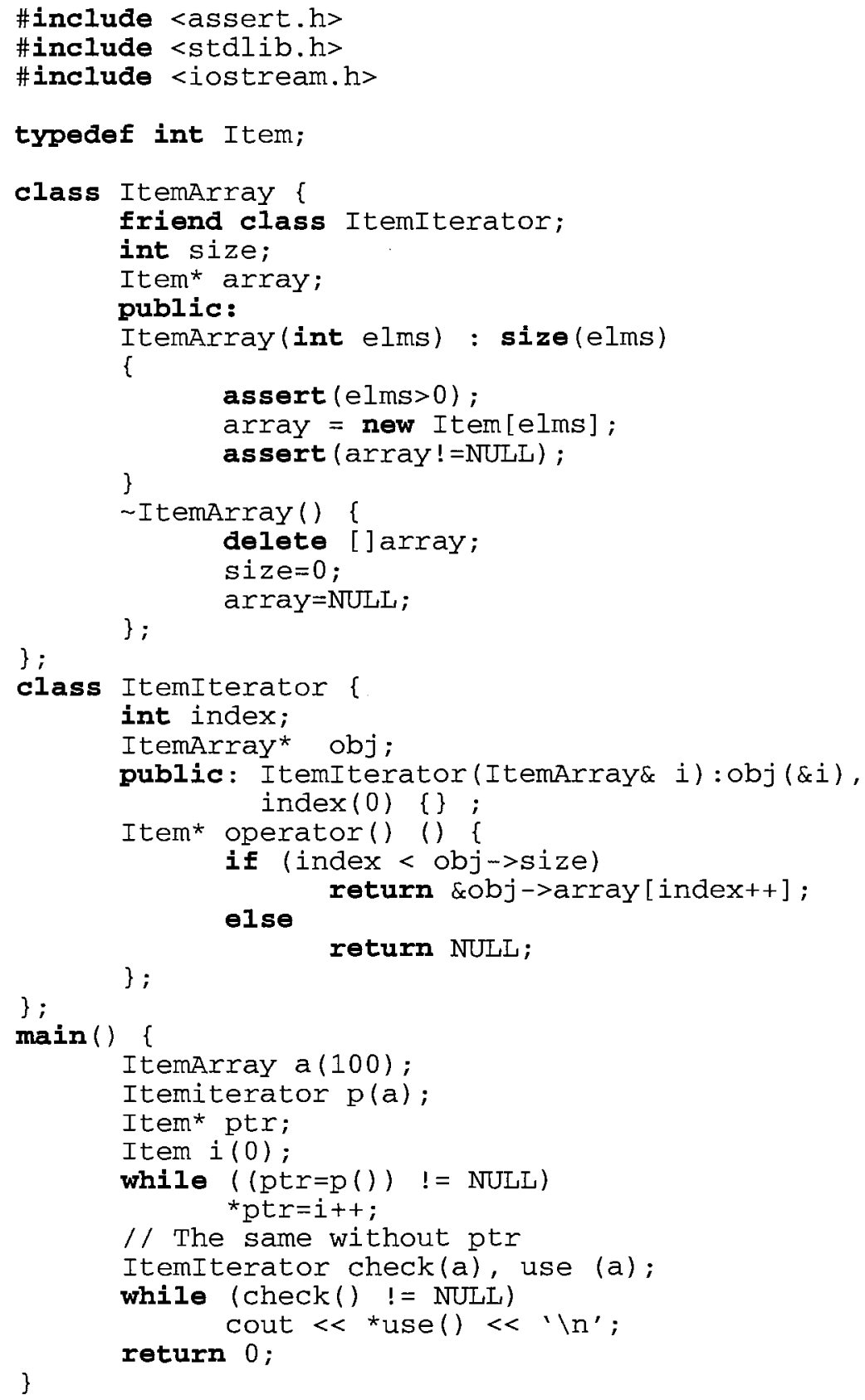

Figure 59. A C++ program that uses iterators 
InsertStructureName(ID) is used to insert structure names into the list. InsertEnumName(ID) is used to insert enumeration names into the list. Insert TemplateName(ID) is used to insert template names into the list. InsertTypeDefName(ID) is used to insert type definition names into the list.

For example, all types of the program in Figure 59 can be stored in the database as shown in Figure 61.

\begin{tabular}{|l|l|}
\hline \multicolumn{1}{|c|}{ Type } & Group \\
\hline bool & Basic \\
char & Basic \\
unsigned & Basic \\
long & Basic \\
short & Basic \\
int & Basic \\
float & Basic \\
double & Basic \\
void & Basic \\
List of Enum Names & Enum \\
List of Structure Names & Structure \\
List of Class Names & Class \\
List of Template Names & Template \\
List of TypeDef Names & TypeDef \\
\hline
\end{tabular}

Figure 60. Show the database of Types used in $\mathrm{C}++$

\begin{tabular}{|l|l|}
\hline \multicolumn{1}{|c|}{ Type } & \multicolumn{1}{c|}{ Group } \\
\hline int & Basic \\
Item & TypeDef \\
ItemArray & Class \\
ItemIterator & Class \\
\hline
\end{tabular}

Figure 61. Show how the database stores Types of the program in Figure 59 


\section{C.2.2 Declarations}

Before a name (identifier) can be used in a $\mathrm{C}++$ program, it must be declared. That is, its type must be specified to inform the compiler to what kind of entity the name refers. The data structure of Declarations is used to store all variables declared in the program with their characteristic and scopes. It is designed as shown in Figure 62 . For example, for the program in Figure 59, all variables can be stored in the database as shown in Figure 63.

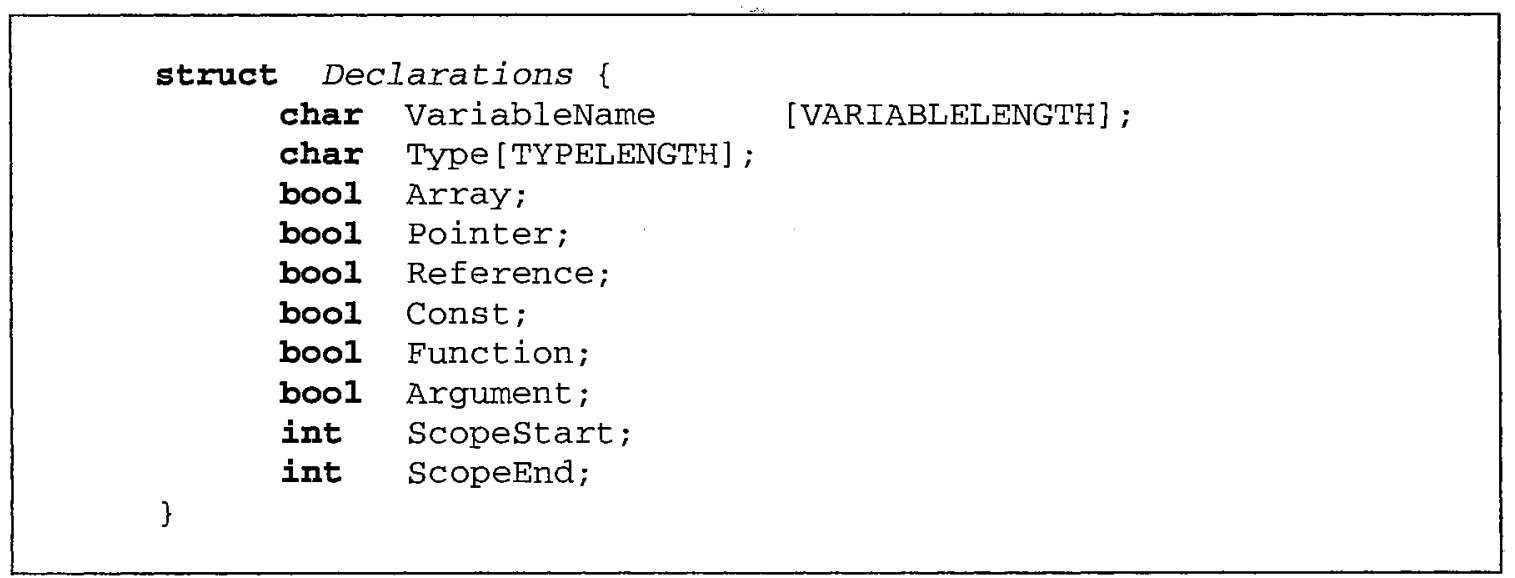

Figure 62. Data structure of Declarations

\begin{tabular}{|c|c|c|c|c|c|c|c|c|c|}
\hline $\begin{array}{c}\text { Variable } \\
\text { Name }\end{array}$ & Type & Array & $\begin{array}{c}\text { Poin- } \\
\text { ter }\end{array}$ & $\begin{array}{c}\text { Refer- } \\
\text { ence }\end{array}$ & Const & $\begin{array}{c}\text { Func- } \\
\text { tion }\end{array}$ & $\begin{array}{c}\text { Argu- } \\
\text { ment }\end{array}$ & $\begin{array}{c}\text { Scope } \\
\text { Start }\end{array}$ & $\begin{array}{c}\text { Scope } \\
\text { End }\end{array}$ \\
\hline Size & int & F & $\mathrm{F}$ & F & F & $\mathrm{F}$ & F & 7 & 21 \\
\hline Array & Item & F & $\mathrm{T}$ & F & F & $\mathrm{F}$ & $\mathrm{F}$ & 8 & 21 \\
\hline Elms & int & F & $\mathrm{F}$ & $F$ & F & $\mathrm{F}$ & $\mathrm{T}$ & 10 & 15 \\
\hline Index & int & $\mathrm{F}$ & $\mathrm{F}$ & $\mathrm{F}$ & F & $\mathrm{F}$ & $\mathrm{F}$ & 23 & 32 \\
\hline Obj & ItemArray & $\mathrm{F}$ & $\mathrm{T}$ & $F$ & $\mathrm{~F}$ & $\mathrm{~F}$ & $\mathrm{~F}$ & 24 & 32 \\
\hline I & ItemArray & $\mathrm{F}$ & $\mathrm{F}$ & $\mathrm{T}$ & $\mathrm{F}$ & $\mathrm{F}$ & $\mathrm{T}$ & 25 & 25 \\
\hline Operator & Item & F & $\mathrm{T}$ & $\mathrm{F}$ & $\mathrm{F}$ & $\mathrm{T}$ & $\mathrm{F}$ & 1 & 45 \\
\hline A & ItemArray & F & F & F & $\mathrm{F}$ & $\mathrm{F}$ & F & 34 & 45 \\
\hline $\mathbf{P}$ & ItemArray & F & $\mathrm{F}$ & $\mathrm{F}$ & $\mathrm{F}$ & $\mathrm{F}$ & $\mathrm{F}$ & 35 & 45 \\
\hline Ptr & Item & $F$ & $\mathrm{~F}$ & $\mathrm{~F}$ & $\mathrm{~F}$ & $\mathrm{~F}$ & $\mathrm{~F}$ & 36 & 45 \\
\hline I & Item & $\mathrm{F}$ & $\mathrm{F}$ & $\mathrm{F}$ & $\mathrm{F}$ & $\mathrm{F}$ & F & 37 & 45 \\
\hline
\end{tabular}

Figure 63. Show how the database stores Declarations of the program in Figure 59 
Where the elements in the field named Type is one of the elements contained in the field named Type in Figure 58. Field named Array, Pointer, Reference, Const, Function, and Argument are used to specify the kinds of each variable in the field of VariableName. Function InsertDeclaration(ID) is used to insert the variables into the database as shown in Figure 63. Function InsertTypes(ID) in Section C.2.1 is called to store all built-in and user-defined types also.

Function Scopestart(ID) and ScopeEnd(ID) are used to determined the scope of each variable name. See Section C.2.3 for more information.

\section{C.2.3 Scope}

A declaration introduces a name into a scope; that is, a name can be used only in a specific part of the program text. Figure 64 shows the example of the scopes. Function Scopestart(ID) and ScopeEnd(ID) are used to determined the scope of each identifier and store it in the database as shown in Figure 63.

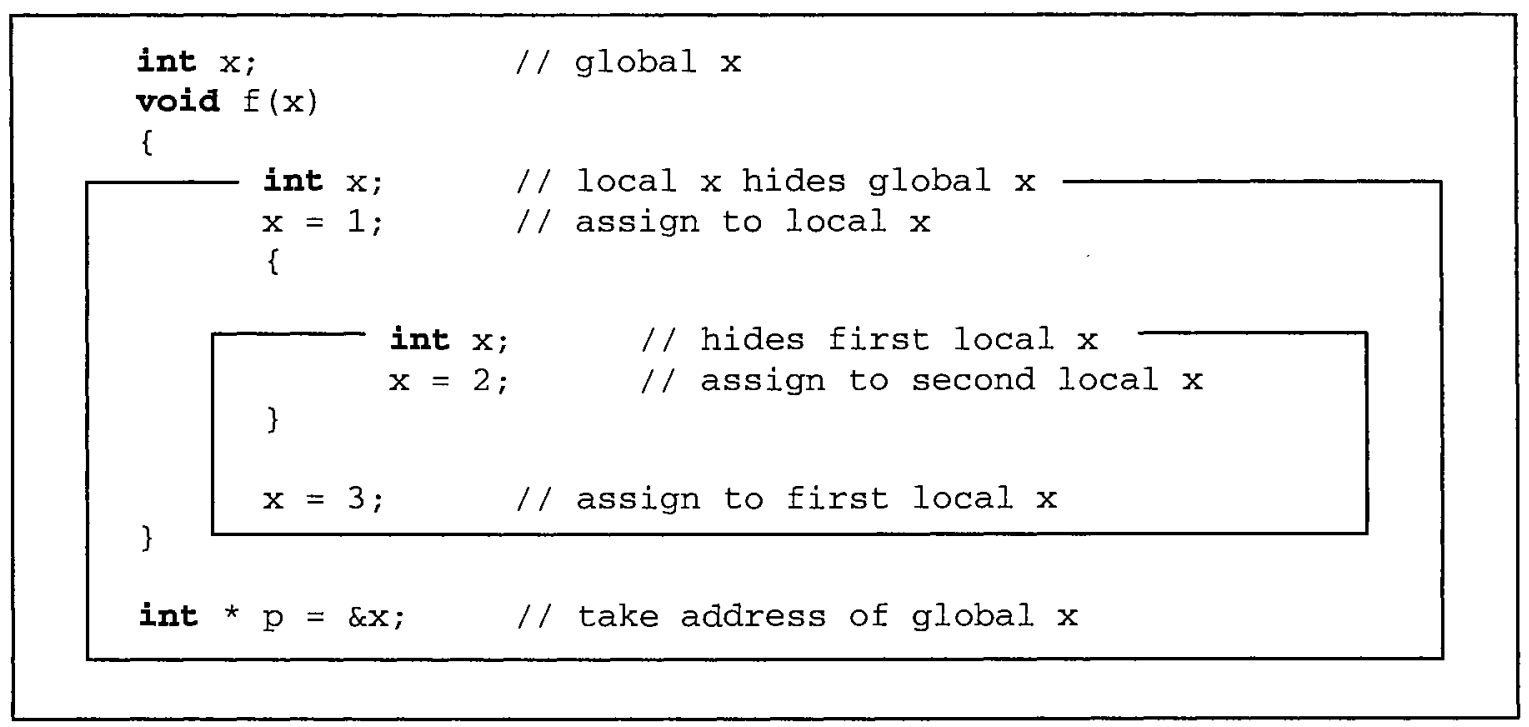

Figure 64. Scopes of variable $\mathrm{x}$ as a global, local, and second local 


\section{C.2.4 Typedef}

A declaration prefixed by the keyword typedef declares a new name for the type rather than a new variable of the given type. The data structure of Typedef is used to store the real types of the types defined by typedef statement. Its data structure is shown in Figure 65. The field named Type in Figure 65 is related to the field named Type in Figure 58. For example, the part of the program in Figure 66 can be stored in the database as shown in Figure 67.

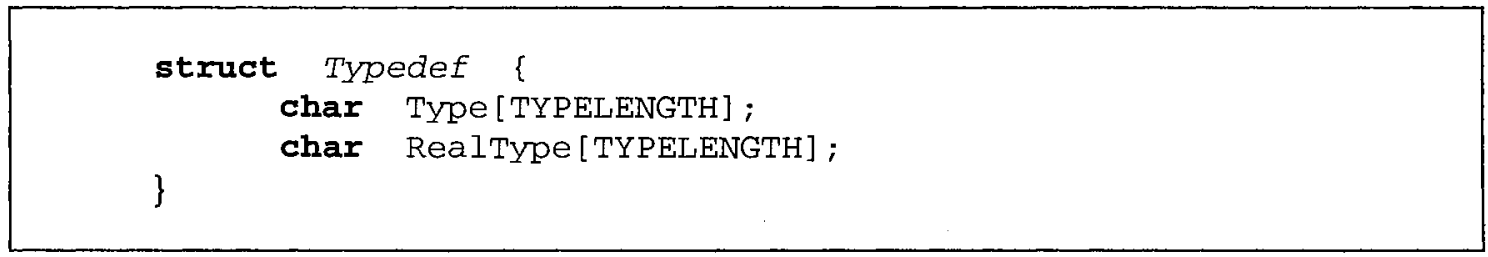

Figure 65. Data structure of Typedef

\begin{tabular}{lll|} 
typedef & short & int16; \\
typedef & int & int32; \\
typedef & long & int64; \\
typedef & char * & Pchar; \\
Pchar & P1, P2; & \\
char & P3 = P1; &
\end{tabular}

Figure 66. A program segment that uses typedef

\begin{tabular}{|l|l|}
\hline Type & Real Type \\
\hline Pchar & char* \\
int16 & short \\
int32 & int \\
int64 & long \\
\hline
\end{tabular}

Figure 67. Show how the database stores Typedef defined by typedef in Figure 66 


\section{C.3 Pointers, Arrays, Constant, References, and Structures}

\section{C.3.1 Pointers}

For a type $\mathrm{T}, \mathrm{T}^{*}$ is the type pointer to $\mathrm{T}$. That is, a variable of type $\mathrm{T}^{*}$ can hold the address of an object of type T. The data structure named Pointers is used to store the variables pointed to. The field named VariableName in Figure 68 is related to the field VariableName in Figure 62. The function named InsertPointerName(ID) is used to manage Figure 70. For example, the part of the program in Figure 69 can be stored in the database as shown in Figure 70.

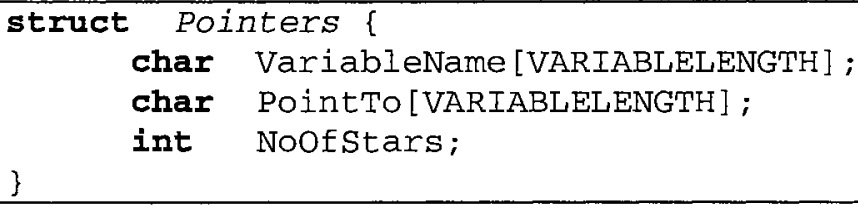

Figure 68. Data structure of Pointers

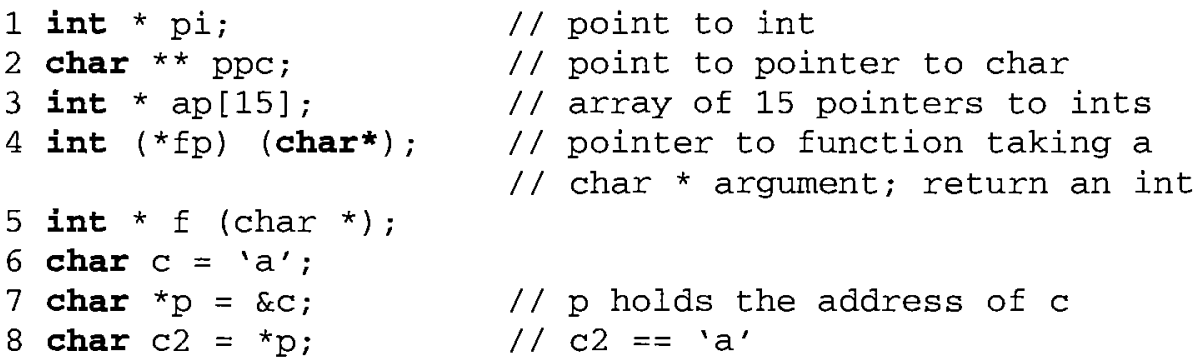

Figure 69. A program segment that uses pointers

Note: We do not care prefixed ' $*$ ' and ' $\&$ ' of variables $p$ and $c$, because their characteristic was stored in Figure 70. 


\begin{tabular}{|c|c|c|}
\hline VariableName & PointTo & NoOfStars \\
\hline $\mathrm{pi}$ & NULI & 1 \\
$\mathrm{ppc}$ & NULI & 2 \\
$\mathrm{ap}$ & NULI & 1 \\
$\mathrm{fp}$ & NULL & 1 \\
$\mathrm{f}$ & NULL & 1 \\
$\mathrm{p}$ & \&c & 1 \\
\hline
\end{tabular}

Figure 70. Show how the database stores Pointers of the part of the program in Figure 69

\begin{tabular}{|c|c|c|}
\hline Line Number & Def(n) & Ref(n) \\
\hline 6 & $\mathrm{c}$ & - \\
7 & $\mathrm{P}$ & $\mathrm{c}$ \\
8 & $\mathrm{c} 2$ & $\mathrm{p}$ \\
\hline
\end{tabular}

Figure 71. Show how the database stores $\operatorname{Def}(n)$ and $\operatorname{Re}(n)$ of the part of the program in Figure 69

\section{C.3.2 Arrays}

For a type $\mathrm{T}, \mathrm{T}[\mathrm{Size}]$ is the type array of size elements of type $\mathrm{T}$. The elements are indexed from 0 to size-1. The data structure named Arrays is used to store all array variables' dimensions. The field named VariableName in Figure 72 is related to the field named VariableName in Figure 62. The function InsertArrayName(ID) is used to manage Figure 74.

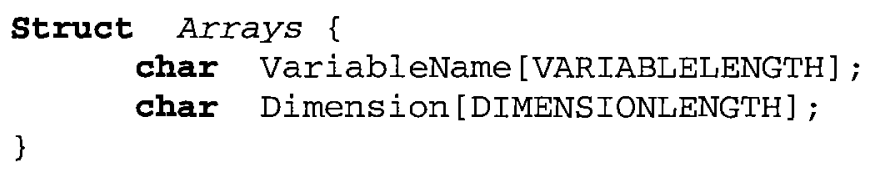

Figure 72. Data structure of Arrays 


$\begin{array}{llll}\text { float } & \mathrm{v}[3] ; & / / & 1 \text { Dimension and size }=3 \\ \text { char } & \mathrm{a}[32][10] ; & / / & 2 \text { dimension and size }=32 \times 10\end{array}$

Figure 73. A program segment that uses arrays

\begin{tabular}{|c|c|}
\hline VariableName & Dimension \\
\hline $\mathrm{v}$ & {$[3]$} \\
$\mathrm{a}$ & {$[32][10]$} \\
\hline
\end{tabular}

Figure 74. Show the data base of Arrays used by the part of the program in Figure 73

\section{C.3.3 Pointers into Arrays}

In $\mathrm{C}++$, pointers and arrays are closely related. The name of array can be used as a pointer to its initial element. Therefore, all variables of these types will be managed by the mixing of Pointers in Section C.3.1 and Arrays in Section C.3.2.

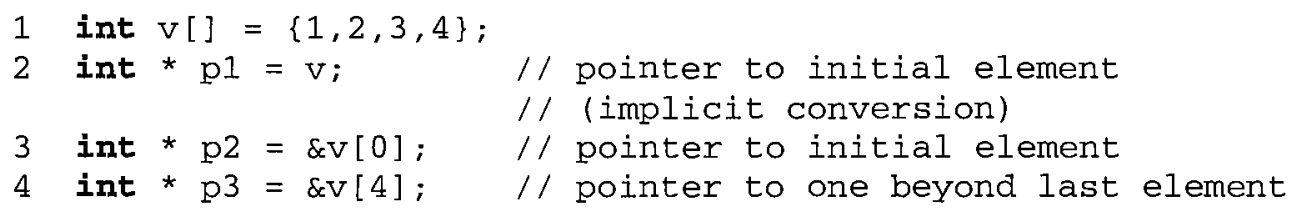

Figure 75. A program segment that uses pointers into arrays

\begin{tabular}{|c|c|c|}
\hline VariableName & PointTo & NoOfStars \\
\hline p1 & $\mathrm{v}$ & 1 \\
p2 & $\& \mathrm{v}[0]$ & 1 \\
p3 & $\& \mathrm{v}[4]$ & 1 \\
\hline
\end{tabular}

Figure 76. Show how the database uses function InsertPointerName(ID) in Section C.3.1 to store variables of the part of the program in Figure 75 


\begin{tabular}{|c|c|}
\hline VariableName & Dimention \\
\hline $\mathrm{v}$ & {$[4]$} \\
\hline
\end{tabular}

Figure 77. Show how the database uses function InsertArrayName(ID) in Section C.3.2 to store variables of the part of the program in Figure 75

\begin{tabular}{|c|c|c|}
\hline Line Number & Def(n) & Ref(n) \\
\hline 1 & $\mathrm{v}$ & - \\
2 & $\mathrm{p} 1$ & $\mathrm{v}$ \\
3 & $\mathrm{p} 2$ & $\mathrm{v}[0]$ \\
4 & $\mathrm{p} 3$ & $\mathrm{v}[4]$ \\
\hline
\end{tabular}

Figure 78. Show how the database stores $\operatorname{Def}(n)$ and $\operatorname{Re} f(n)$ of the part of the program in Figure 75

\section{C.3.4 Constant}

$\mathrm{C}++$ offers the concept of a user-defined constant, a constant, to express the notation that a value doesn't change directly. The datastructure named Declarations in Section C.2.2 is used to manage by using the function named InsertDeclaration(ID), while the data structure named Pointers in Section C.3.1 used to manage their pointers.

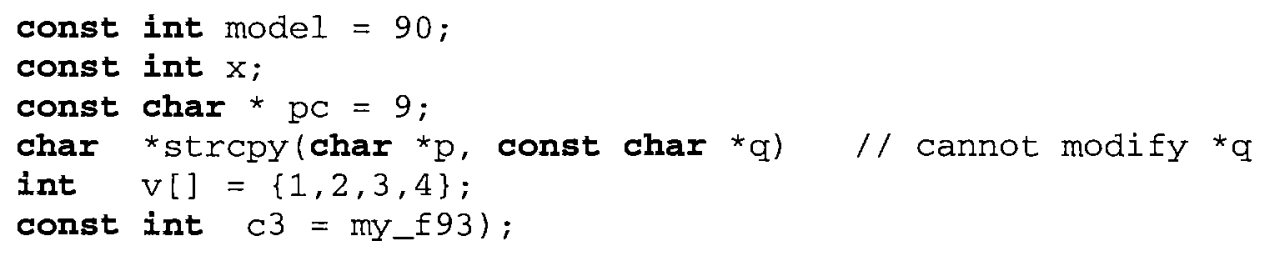

Figure 79. A program segment that uses const 


\begin{tabular}{|c|c|c|c|c|c|c|c|c|c|}
\hline $\begin{array}{c}\text { Variable } \\
\text { Name }\end{array}$ & Type & Array & $\begin{array}{c}\text { Poin- } \\
\text { ter }\end{array}$ & $\begin{array}{c}\text { Refer- } \\
\text { Ence }\end{array}$ & Const & $\begin{array}{c}\text { Func- } \\
\text { tion }\end{array}$ & $\begin{array}{l}\text { Argu- } \\
\text { ment }\end{array}$ & $\begin{array}{c}\text { Scope } \\
\text { Start }\end{array}$ & $\begin{array}{c}\text { Scope } \\
\text { End }\end{array}$ \\
\hline mode1 & int & $F$ & $F$ & F & $\mathrm{T}$ & $F$ & $\mathrm{~F}$ & 1 & 6 \\
\hline $\mathrm{x}$ & int & $F$ & $F$ & $F$ & $\mathrm{~T}$ & $F$ & F & 2 & 6 \\
\hline $\mathrm{pc}$ & char & $F$ & $\mathrm{~T}$ & $F$ & $\mathrm{~T}$ & $F$ & $F$ & 3 & 6 \\
\hline strcpy & char & $\mathrm{F}$ & $\mathrm{T}$ & $F$ & $\mathrm{~T}$ & $\mathrm{~T}$ & F & 4 & 6 \\
\hline $\mathrm{V}$ & int & $\mathrm{T}$ & $F$ & $F$ & $\mathrm{~T}$ & $F$ & F & 5 & 6 \\
\hline$c 3$ & int & $F$ & $F$ & F & $\mathrm{T}$ & $F$ & $F$ & 6 & 6 \\
\hline$p$ & char & $F$ & $\mathrm{~T}$ & $F$ & $F$ & $F$ & $\mathrm{~T}$ & 4 & 6 \\
\hline q & char & $F$ & $\mathrm{~T}$ & $F$ & $\mathrm{~T}$ & $F$ & $\mathrm{~T}$ & 4 & 6 \\
\hline
\end{tabular}

Figure 80. Show how the database stores constant declared in Figure 79

For example, from the part of the program in Figure 79, all variables can be stored in the data base as shown in Figure 80, all pointers are stored in the database as shown in Figure 80, and their $\operatorname{Def}(\mathrm{n})$ and $\operatorname{Re}(\mathrm{n})$ can be determined as shown in Figure 82.

\begin{tabular}{|c|c|c|}
\hline VariableName & PointTo & NoOfStars \\
\hline $\mathrm{pc}$ & 9 & 1 \\
strcpy & NULL & 1 \\
\hline
\end{tabular}

Figure 81. Show how the database uses function InsertPointerName(ID) in section C.3.1 to variables of the part of the program in Figure 79

\begin{tabular}{|c|c|c|}
\hline Line Number & Def(n) & Ref(n) \\
\hline 1 & model & - \\
2 & pc & - \\
3 & c3 & my_f \\
\hline
\end{tabular}

Figure 82. Show how the database stores $\operatorname{Def}(n)$ and $\operatorname{Ref}(n)$ of the part of the program in Figure 79 


\section{C.3.5 References}

A reference is an alternative name for an object. The main use of references is for specifying arguments and returns values for functions in general and for overloaded operators in particular. The notation $\mathrm{X} \&$ means reference to $\mathrm{X}$. In this design we will see a reference variable as copied variable as shown in Figure 85 which is managed by the function named InsertReferenceName(ID).

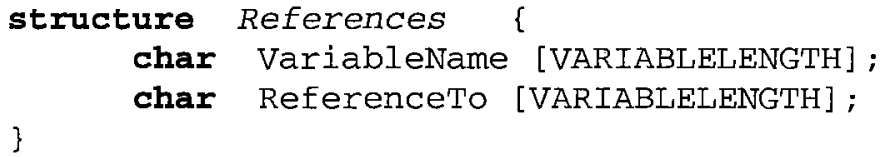

Figure 83. Data structure of References

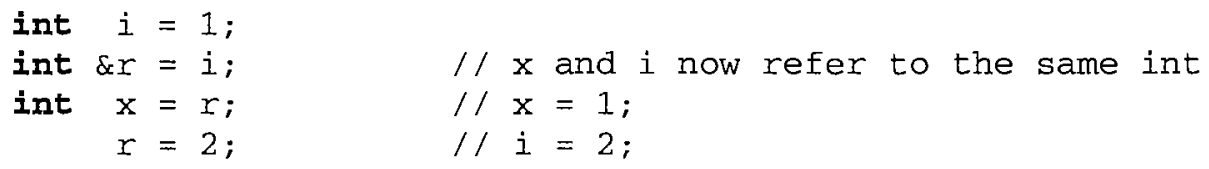

Figure 84. A program segment that uses references

\begin{tabular}{|c|c|}
\hline VariableName & ReferenceTo \\
\hline $\mathbf{r}$ & $\mathrm{i}$ \\
\hline
\end{tabular}

Figure 85. Show how the database uses function InsertReferenceName(ID) to store variables of the part of the program in Figure 84

\section{C.3.6 Pointer to Void}

A pointer of any type of object can be assigned to a variable of type void*, a void* can be assigned to another void*, void*s can be compared for equality and 
inequality, and a void* can be explicitly converted to another type. In this design, functions named InsertDeclarationName(ID) in Section C.3.2 and InsertPointerName(ID) in Section C.3.1 are used to manage their variables.

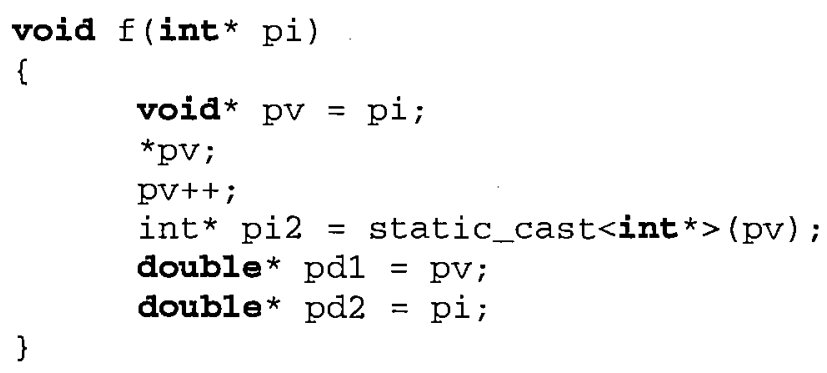

Figure 86. A program segment that uses pointer to void

\section{C.3.7 Structures}

An array is an aggregate of elements of the same type. A struct is an aggregate of elements of (nearly) arbitrary types. In this design, the data structure of Structures used to store all structures defined in the program is shown in Figure 88. Functions InsertStructureName(ID) and InsertDeclaration(ID) in section C.2.2 are used to manage as shown in Figure 90.

\begin{tabular}{|c|c|c|}
\hline VariableName & PointTo & NoOfStars \\
\hline pi & NULL & 1 \\
pv & pi & 1 \\
pi2 & static_cast & 1 \\
pd1 & pv & 1 \\
pd2 & pi & 1 \\
\hline
\end{tabular}

Figure 87. Show how the database uses function InsertPointerName(ID) in section C.3.1 to store variables of the part of the program in Figure 86 


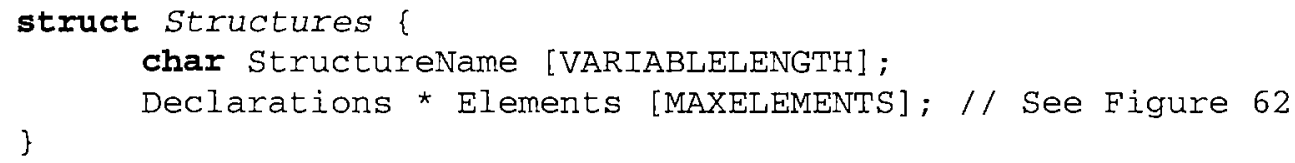

Figure 88. Data structure of Structures

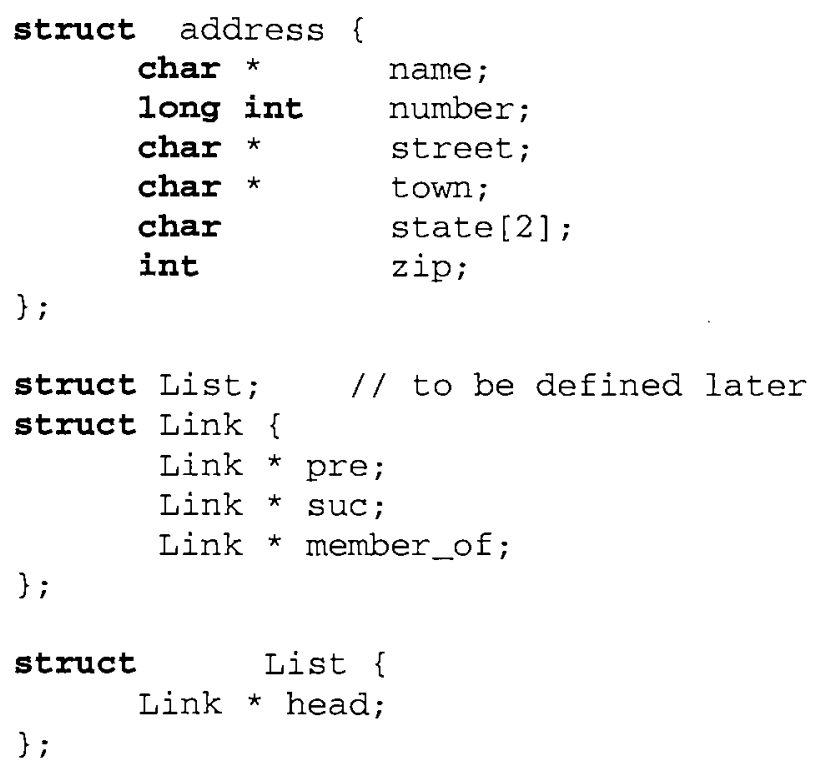

Figure 89. A program segment that uses structures

\section{C.4 Operators}

The function named $\operatorname{Def}(\mathrm{n})$ is used to determine the set of variables whose values may be defined at line number $n$, while the function named $\operatorname{Re} f(n)$ is used to determine the set of variables whose values may be referenced at line number $\mathrm{n}$, as shown in Figure 91. 


\begin{tabular}{|c|c|}
\hline $\begin{array}{c}\text { Structure } \\
\text { Name }\end{array}$ & Elements \\
\hline address & \\
\hline Link & \\
\hline List & \\
\hline & \\
\hline
\end{tabular}

\begin{tabular}{|l|c|c|c|c|c|c|c|c|c|}
\hline $\begin{array}{c}\text { Variable } \\
\text { Name }\end{array}$ & Type & Array & $\begin{array}{c}\text { Poin- } \\
\text { ter }\end{array}$ & $\begin{array}{c}\text { Refer- } \\
\text { ence }\end{array}$ & Const & $\begin{array}{c}\text { Func- } \\
\text { tion }\end{array}$ & $\begin{array}{c}\text { Argu- } \\
\text { ment }\end{array}$ & $\begin{array}{c}\text { Scope } \\
\text { Start }\end{array}$ & $\begin{array}{c}\text { Scope } \\
\text { End }\end{array}$ \\
\hline name & char & $\mathrm{F}$ & $\mathrm{T}$ & $\mathrm{F}$ & $\mathrm{F}$ & $\mathrm{F}$ & $\mathrm{F}$ & - & - \\
number & long & $\mathrm{F}$ & $\mathrm{F}$ & $\mathrm{F}$ & $\mathrm{F}$ & $\mathrm{F}$ & $\mathrm{F}$ & - & - \\
street & char & $\mathrm{F}$ & $\mathrm{T}$ & $\mathrm{F}$ & $\mathrm{F}$ & $\mathrm{F}$ & $\mathrm{F}$ & - & - \\
town & char & $\mathrm{F}$ & $\mathrm{T}$ & $\mathrm{F}$ & $\mathrm{F}$ & $\mathrm{F}$ & $\mathrm{F}$ & - & - \\
state & char & $\mathrm{T}$ & $\mathrm{F}$ & $\mathrm{F}$ & $\mathrm{F}$ & $\mathrm{F}$ & $\mathrm{F}$ & - & - \\
zip & int & $\mathrm{F}$ & $\mathrm{F}$ & $\mathrm{F}$ & $\mathrm{F}$ & $\mathrm{F}$ & $\mathrm{F}$ & - & - \\
\hline
\end{tabular}

\begin{tabular}{|l|c|c|c|c|c|c|c|c|c|}
\hline $\begin{array}{c}\text { Variable } \\
\text { Name }\end{array}$ & Type & Array & $\begin{array}{c}\text { Poin- } \\
\text { ter }\end{array}$ & $\begin{array}{c}\text { Refer- } \\
\text { ence }\end{array}$ & Const & $\begin{array}{c}\text { Func- } \\
\text { tion }\end{array}$ & $\begin{array}{c}\text { Argu- } \\
\text { ment }\end{array}$ & $\begin{array}{c}\text { Scope } \\
\text { Start }\end{array}$ & $\begin{array}{c}\text { Scope } \\
\text { End }\end{array}$ \\
\hline \multirow{2}{*}{ pre } & Link & $\mathrm{F}$ & $\mathrm{T}$ & $\mathrm{F}$ & $\mathrm{F}$ & $\mathrm{F}$ & $\mathrm{F}$ & - & - \\
member_of & Link & $\mathrm{F}$ & $\mathrm{T}$ & $\mathrm{F}$ & $\mathrm{F}$ & $\mathrm{F}$ & $\mathrm{F}$ & - & - \\
& Link & $\mathrm{F}$ & $\mathrm{T}$ & $\mathrm{F}$ & $\mathrm{F}$ & $\mathrm{F}$ & $\mathrm{F}$ & - & - \\
\hline
\end{tabular}

\begin{tabular}{|l|c|c|c|c|c|c|c|c|c|}
\hline $\begin{array}{c}\text { Variable } \\
\text { Name }\end{array}$ & Type & Array & $\begin{array}{c}\text { Poin- } \\
\text { ter }\end{array}$ & $\begin{array}{c}\text { Refer- } \\
\text { ence }\end{array}$ & Const & $\begin{array}{c}\text { Func- } \\
\text { tion }\end{array}$ & $\begin{array}{c}\text { Argu- } \\
\text { ment }\end{array}$ & $\begin{array}{c}\text { Scope } \\
\text { Start }\end{array}$ & $\begin{array}{c}\text { Scope } \\
\text { End }\end{array}$ \\
\hline head & Link & $\mathrm{F}$ & $\mathrm{T}$ & $\mathrm{F}$ & $\mathrm{F}$ & $\mathrm{F}$ & $\mathrm{F}$ & - & - \\
\hline
\end{tabular}

Figure 90. Show how the database stores Structures of the part of the program in Figure 89 


\begin{tabular}{|c|c|c|c|c|}
\hline Description & Grammar & Statement & $\operatorname{Def}(n)$ & $\operatorname{Ref}(n)$ \\
\hline post increment & lvalue ++ & $\mathrm{I}++$ & I & $\mathrm{I}$ \\
\hline post decrement & lvalue -- & d-- & $\mathrm{d}$ & $\mathrm{d}$ \\
\hline size of object & sizeof (expr) & $a=$ sizeof(int) & $\mathrm{a}$ & sizeof \\
\hline pre increment & ++ lvalue & $++\mathrm{I}$ & $I$ & $\mathrm{I}$ \\
\hline pre decrement & -- value & $--d$ & $\mathrm{~d}$ & $\mathrm{~d}$ \\
\hline complement & $\sim \operatorname{expr}$ & $\mathrm{t}=\sim \mathrm{e}$ & $t$ & $\mathrm{e}$ \\
\hline not & !expr & $t=! e$ & $t$ & $\mathrm{e}$ \\
\hline unary minus & - expr & $t=-e$ & $\mathrm{t}$ & $\mathrm{e}$ \\
\hline unary plus & + expr & $\mathrm{t}=+\mathrm{e}$ & $\mathrm{t}$ & $\mathrm{e}$ \\
\hline address of & \& lvalue & $\& \mathrm{t}=\mathrm{e}$ & $\mathrm{t}$ & $\mathrm{e}$ \\
\hline dereference & * expr & $*_{\mathrm{t}}=\mathrm{e}$ & $\mathrm{t}$ & $\bar{e}$ \\
\hline create(allocate) & new [type] & $\mathrm{t}=$ new [Type] & $\mathrm{t}$ & e, Type \\
\hline multiply & expr * expr & $\mathrm{t}=\mathrm{e} 1 * \mathrm{e} 2$ & $\mathrm{t}$ & $\mathrm{e} 1, \mathrm{e} 2$ \\
\hline divide & expr / expr & $\mathrm{t}=\mathrm{e} 1 / \mathrm{e} 2$ & $t$ & $\mathrm{e} 1, \mathrm{e} 2$ \\
\hline modulo (remainder) & expr \% expr & $\mathrm{t}=\mathrm{e} 1 \% \mathrm{e} 2$ & $\mathrm{t}$ & $\mathrm{e} 1, \mathrm{e} 2$ \\
\hline add (plus) & expr + expr & $t=e 1+e 2$ & $\mathrm{t}$ & $\mathrm{e} 1, \mathrm{e} 2$ \\
\hline subtract (minus) & expr - expr & $\mathrm{t}=\mathrm{e} 1-\mathrm{e} 2$ & $\mathrm{t}$ & $\mathrm{e} 1, \mathrm{e} 2$ \\
\hline shift left & expr $<<\operatorname{expr}$ & $\mathrm{t}=\mathrm{e} 1<<\mathrm{e} 2$ & $t, \mathrm{e} 1$ & $\mathrm{e} 2$ \\
\hline shift right & expr $\gg$ expr & $\mathrm{t}=\mathrm{e} 1>>\mathrm{e} 2$ & $\mathrm{t}, \mathrm{e} 1$ & $\mathrm{e} 2$ \\
\hline less than & expr $<$ expr & $\mathrm{b}=\mathrm{e} 1<\mathrm{e} 2$ & $\mathrm{~b}$ & $\mathrm{e} 1, \mathrm{e} 2$ \\
\hline less than or equal & expr $<=$ expr & $\mathrm{b}=\mathrm{e} 1<=\mathrm{e} 2$ & $\mathrm{~b}$ & $\mathrm{e} 1, \mathrm{e} 2$ \\
\hline greather than & expr $>$ expr & $\mathrm{b}=\mathrm{e} 1>\mathrm{e} 2$ & $\mathrm{~b}$ & $\mathrm{e} 1, \mathrm{e} 2$ \\
\hline greather than or equal & expr $>=$ expr & $\mathrm{b}=\mathrm{e} 1>=\mathrm{e} 2$ & $\mathrm{~b}$ & $\mathrm{e} 1, \mathrm{e} 2$ \\
\hline equal & expr $==\operatorname{expr}$ & $\mathrm{b}=\mathrm{e} 1==\mathrm{e} 2$ & $\mathrm{~b}$ & $\mathrm{e} 1, \mathrm{e} 2$ \\
\hline not equal & expr $!=\operatorname{expr}$ & $\mathrm{b}=\mathrm{e} 1 !=\mathrm{e} 2$ & $\mathrm{~b}$ & $\mathrm{e} 1, \mathrm{e} 2$ \\
\hline bitwise AND & expr \& expr & $\mathrm{b}=\mathrm{e} 1 \& \mathrm{e} 2$ & $\mathrm{~b}$ & $\mathrm{e} 1, \mathrm{e} 2$ \\
\hline bitwise exclusive OR & expr $\wedge$ expr & $\mathrm{b}=\mathrm{e} 1^{\wedge} \mathrm{e} 2$ & $\mathrm{~b}$ & $\mathrm{e} 1, \mathrm{e} 2$ \\
\hline bitwise inclusive OR & \begin{tabular}{l|l} 
expr & expr \\
\end{tabular} & $\mathrm{b}=\mathrm{e} 1 \mid \mathrm{e} 2$ & $\mathrm{~b}$ & $\mathrm{e} 1, \mathrm{e} 2$ \\
\hline logical AND & expr \&\& expr & $\mathrm{b}=\mathrm{b} 1 \& \& \mathrm{~b} 2$ & $\mathrm{~b}$ & b1, b2 \\
\hline logical inclusive $\mathrm{OR}$ & expr $\|$ expr & $\mathrm{b}=\mathrm{b} 1 \| \mathrm{b} 2$ & $\mathrm{~b}$ & $\mathrm{~b} 1, \mathrm{~b} 2$ \\
\hline simple assig nment & lvalue $=$ expr & $\mathrm{t}=\mathrm{e}$ & $\mathrm{t}$ & $\mathrm{e}$ \\
\hline multiply and assign & lvalue $*=\operatorname{expr}$ & $\mathrm{t} *=\mathrm{e}$ & $\mathrm{t}$ & $\mathrm{t}, \mathrm{e}$ \\
\hline divide and assign & lvalue $/=\operatorname{expr}$ & $t /=e$ & $\mathrm{t}$ & $\mathrm{t}, \mathrm{e}$ \\
\hline modulo and assign & lvalue $\%=$ expr & $\mathrm{t} \%=\mathrm{e}$ & $\mathrm{t}$ & $\mathrm{t}, \mathrm{e}$ \\
\hline add and assign & lvalue $+=\operatorname{expr}$ & $t+=e$ & $\mathrm{t}$ & $t, e$ \\
\hline subtract and assign & lvalue $-=\operatorname{expr}$ & $\mathrm{t}-=\mathrm{e}$ & $\mathrm{t}$ & $t, \mathrm{e}$ \\
\hline shift left and assign & Ivalue $<<=$ expr & $\mathrm{t} \ll<=\mathrm{e}$ & $\mathrm{t}$ & $t, e$ \\
\hline shift right and assign & lvalue $>>=$ expr & $t>>=e$ & $\mathrm{t}$ & $\mathrm{t}, \mathrm{e}$ \\
\hline AND and assign & lvalue $\&=\operatorname{expr}$ & $\mathrm{t} \&=\mathrm{e}$ & $\mathrm{t}$ & $\mathrm{t}, \mathrm{e}$ \\
\hline inclusive OR and assign & 1value $=$ expr & $\mathrm{t} H=\mathrm{e}$ & $\mathbf{t}$ & $\mathrm{t}, \mathrm{e}$ \\
\hline exclusive $O R$ and assign & lvalue $\wedge^{\wedge}=\operatorname{expr}$ & $\mathrm{t}^{\wedge}=\mathrm{e}$ & $\mathbf{t}$ & $t, e$ \\
\hline
\end{tabular}

Figure 91. Show how to determine the set of variables by using functions $\operatorname{Def}(n)$ and $\operatorname{Ref}(n)$ 


\section{APPENDIX D}

\section{SOFTWARE SPECIFICATION}

\section{D.1 Introduction}

The main purpose of software specification is used to define the functionality of $\mathrm{C}++$ Debug and constraints on its operation, plan the system development process, develop validation tests for the system, and help understand the system and the relationships between its parts.

\section{D.2 General Description}

$\mathrm{C}++$ Debug is an interactive debugging tool designed to function as a utility program of the UNIX system. C++Debug is developed based on slicing and dicing techniques. In order for $\mathrm{C}++$ Debug to be more powerful, dynamic slicing rather than static slicing is chosen for implementation. C++Debug was designed in a way to allow ease and convenience on part of the user. Using $\mathrm{C}++$ Debug, the user can interact directly with the computer in locating errors in a program. Menus are provided to allow the user to select any one of a number of functions (Slice, Dice, Help, etc.) supported by C++Debug. 


\section{D.3 Specific Requirements}

Functional and non-functional requirements are introduced in this part. Functional requirements provide how the system react to particular inputs, behave in particular situations, and explicitly state what the system should not do [Sommerville 01]. Non-functional requirements are about constraints such as timing constraints, constraints on the development process, standards, etc.

\section{D.3.1 Functional Requirements}

Function requirements describe services provided for the user by using natural language with cross-references to requirement specifications [Sommerville 94].

\subsubsection{General}

\subsubsection{Name}

$\mathrm{C}++$ Debug

Rational:

$\mathrm{C}++$ Debug is a slicing and dicing based debugging tool for $\mathrm{C}++$.

\subsubsection{Purpose}

This project develops an interactive debugging tool, called C++Debug, Rational: for debugging a $\mathrm{C}++$ language program.

$\mathrm{C}++$ Debug is designed to function as a utility program of the UNIX system and is developed based on slicing and dicing techniques.

\subsubsection{Hardware and Software}

$\mathrm{C}++$ Debug runs under UNIX machine.

Rational:

The SUN machine locates on the second floor of the Computer Science Building.

\subsubsection{Program Slicing}

\subsubsection{ANSI C++}


Can be used with every command and every instruction of ANSI C++ based on UNIX environment.

Rational:

$\mathrm{C}++$ Debug has to generate a program slice for every user program that uses ANSI C++ based on UNIX environment to implement the program.

\subsubsection{Automatic}

Program slices can be found automatically by a method used to Rational: decompose programs through analyzing their data flow and control flow.

$\mathrm{C}++$ Debug automatically generates program slices.

4.2.1.3 Eighty percent of A program slice must be smaller size than that of the original program.

Rational:

This is because there always at least one slice, that is, program itself. As a consequence, when slicing at a variable of interest, the size of the resulting program slice is generally smaller than that of the original program.

\subsubsection{Property Consistency}

Program slice can be executed independently of one another.

Rational:

The smaller size of the program slice is a $\mathrm{C}++$ program that still maintains all aspects of the original program behavior with respect to the criterion variable.

\subsubsection{Produces Exactly one projection}

Each slice produces exactly one projection of the original program's behavior.

Rational:

The smaller size of the program slice must still maintain all aspects of the original program behavior with respect to the criterion variable.

\subsubsection{Reduction}

The slice must have been obtained from the original program by statement deletion.

Rational:

The idea of a program slicing is to focus on the statements that have something to do with a variable of interest (criterion variable), with those statements that are unrelated being omitted.

\subsubsection{Dynamic Slicing}

\subsubsection{Computation}


Rational:

$\mathrm{C}++$ Debug generates a dynamic program slice by computing from the trajectory of the original source program.

Contradict with static slicing, which generates a static program slice directly from the original source program.

\subsubsection{Arrays and Fields}

$\mathrm{C}++$ Debug treats array elements and fields in dynamic records as individual variables.

Rational:

Dynamic slicing characteristics [Korel 90].

\subsubsection{Size Comparing with Static Slicing}

Dynamic slicing yields a program slice of generally smaller size than that Rational: of static slicing, or in the worst case, of equal size to that of static slicing.

The runtime handling of arrays and pointer variables helps to reduce the size of the slice.

4.2.3.4 \# lines of executable path of the original source program at least 5,000 lines of the executable path of the original codes can be computed by $\mathrm{C}++$ Debug.

Rational:

To make sure that it can work with for any small run-time programs, medium run-time programs and any run-time modules.

\subsubsection{Dicing}

\subsubsection{Computation of a Variable}

If the computation of a variable, $\mathrm{V}$, depends on the computation of another variable, $W$, then when ever $W$ has an incorrect value, so does $V$.

Rational:

From dicing characteristics [Lyle 84].

4.2.4.2 Using the dicing technique, $\mathrm{C}++$ Debug can then be used to compare two or more slices resulting from the program slicing technique to identify the set of statements that are likely to contain an error.

Rational:

From dicing characteristics [Lyle 84].

\subsubsection{Time Complexity}

\subsubsection{Dynamic Slicing}

Time to compute the program slice is less than 2 minutes at 5,000 lines of the executable path of the original codes. 
Rational:

Protected from infinite loops.

\subsubsection{Static Slicing}

Time to compute the program slice is less than 2 minutes at 5,000 lines of Rational: the original codes.

Protected from infinite loops.

\subsubsection{Dicing}

For two variables and 1000 lines of program slices the time to find the Rational: error line is less than 30 seconds.

Users cannot wait for a long time.

\subsubsection{Space Complexity}

4.2.6.1 Size of the machine code.

After compiled, the total size of machine code is not more than $100 \mathrm{k}$ Rational: byte.

Comparing with the other debugger e.g. SDB, DBX etc.

\subsubsection{Memory Space}

While executing, $\mathrm{C}++$ Debug can use the total memory in the system e.g. Rational: stack, heap, code etc., not more than 1 m-byte.

If $\mathrm{C}++$ Debug uses a small primary and secondary storage, it can be used on a small machine.

4.2.7 Single-user and Multi-user

$\mathrm{C}++$ Debug can be used for both single-user and multi-user modes. Rational:

$\mathrm{C}++$ Debug has been designed to have no critical section, shared memory and shared process, but it was designed to run independently like a UNIX utility command.

\subsubsection{GUI}

GUI's menus are provided by $\mathrm{C}++$ Debug to allow the users to select any one of the functions of slice, dice, help, etc.

Rational:

To make system user-friendly.

\subsubsection{Software Metrics}

Following by Cyclomatic complexity theory 


\section{D.3.2 Non-function Requirements}

\subsubsection{User Interface}

Although GUI's menus are provided by $\mathrm{C}++$ Debug to allow the user to select any one of the functions i.e. slice, dice, help, etc., for other function we cannot specify one. However, $\mathrm{C}++$ Debug must be designed to user-friendly.

\subsubsection{System Cost}

In order to compete with other debugger tools in the market, the price of $\mathrm{C}++$ Debug at full functions should not more than $49 \$$. So the total cost of $\mathrm{C}++$ Debug project should not more than 10,000 dollars.

\subsubsection{Software Size}

Although a large program can be run in UNIX environment, however, the size of $\mathrm{C}++$ Debug should not be more than $100 \mathrm{k}$-byte. The reason is that average size of other debuggers e.g. DBX, SDB are not more than 100k bytes.

\subsubsection{Reliability}

After delivering $\mathrm{C}++$ Debug to the customer, the number of errors must exist not more than 3 times a month. And the existing errors must be corrected in 1 week since it has been found. 


\section{APPENDIX E}

\section{TESTING AND EVALUATION}

\section{E.1 Introduction}

$\mathrm{C}++$ Debug was designed to function as a utility program of the UNIX system and was developed based on slicing and dicing techniques. After $\mathrm{C}++$ Debug was implemented, testing was conducted to ensure that each unit met its specification (unit testing), and to ensure that the software requirements had been met integration and system testing was done. Testing is the primary means for showing that the implementation has the requisite functionality and satisfies other non-functional properties [McDermid 93]. Testing and other forms of verification and validation are important at all stages of the software development process. In order to know how $\mathrm{C}++$ Debug can be used to enhance the debugging process, evaluation was introduced.

\section{E.2 Testing}

\section{E.2.1 Black and White Testing}

Black and white testing was used to test $\mathrm{C}++$ Debug. In black-box testing, the internal structure and behavior of a system is not considered when the test data is selected 
[McDermid 93]. Acceptance testing is the testing of a software system to ensure that it meets user requirements (see Appendix D). At this stage the test data is chosen by a careful reading of the requirements specification. In white-box testing, (e.g., unit testing), the internal structure and behavior of a system is considered when the test data is selected. Here a program unit (subroutine, procedure) of $\mathrm{C}++$ Debug was exercised with data, with the aim of ensuring that the code inside the unit implemented its specification. In this form of testing, a major aim is to ensure that a certain proportion of the software structure are exercised, a typical target being the execution of about $85 \%$ of the branches and $100 \%$ of all the statement in a program unit [McDermid 93]. The test data sets have to be developed to maximize the proportion of structural elements being exercised. To do this, the internal structure of a unit has to be examined.

\section{E.2.2 Testing and The Software Life Cycle}

During the various phases of the software life cycle, $\mathrm{C}++$ Debug was tested as follows.

\section{E.2.2.1 Testing and Requirements Analysis}

The major development activities that take place during this phase are the elicitation and clarification of requirements and the subsequent construction of the system specification [McDermid 93]. The major testing activity that occurs during this phase is the derivation of the verification requirements. The $\mathrm{C}++$ Debug requirements are listed in Appendix D. During the latter stages of $\mathrm{C}++$ Debug development, their requirements were converted into system validation tests and acceptance tests. Their tests determine whether a system meets its requirements. For example, 
7.8 When the slice-criterion command is typed with a correct variable name and with a valid trajectory number, the program slice will be generated and stored in the file named "_cpptrace_slice.dat".

This leads to a number of tests as follows:

1. If there exists an invalid variable name, an error message will be displayed.

2. If there exists an invalid trajectory number, an error message will be displayed.

3. If there is no slice, a prompt message "No Slice" will be displayed, otherwise the program slice will be stored in the file named "_cpptrace_slice.dat".

\section{E.2.2.2 Testing and System Design}

There are a number of activities during system or architecture design that are relevant to testing [McDermid 93]. First the verification requirements were expanded so that they would correspond more closely to the individual tests.

A second testing-related activity, which should occur during this phase, is to develop the test coverage matrix. This is a matrix, which relates the expanded verification requirements to the modules, which implement the requirements.

A third activity is the development of the integration test strategy. This involves specifying the order in which the program units are to be added to the system, which is being built. A bottom-up strategy, instead of a top-down strategy, was used to test $\mathrm{C}++$ Debug, because it is easier to detect flaws that occur toward the bottom of a design. For example, using the verification requirement in previous example, we will have the following situation.

The number of tests will be expanded as follows: 
V 7.8/1 When the slice-criterion command is typed, with a user identification $\mathrm{A}$, the error message will be displayed on the originating console.

V 7.8/2 When the slice-criterion command is typed, with a user identification $\mathrm{B}$, the error message will be displayed on the originating console.

V 7.8/3 When the slice-criterion command is typed with a user identification which does not match a user currently logged on, the error message will be displayed on the originating console.

\section{E.2.2.3 Testing and Detailed Design}

The main testing activity that occurs during this phase is the construction of $\mathrm{C}++$ Debug test procedures. A test procedure is a detailed step-by-step set of instructions [McDermid 93]. A test procedure contains details of the software configuration used, the hardware configuration, the location of the job control language commands necessary for carrying out the test, the files containing test data, the expected outcomes of the tasks, and the location of the files that contain the test outcome. For example,

bool UsedVariable( Type Var)

executed the function UsedVariable with parameter Var with type Type. If variable Var is a "used" set, then return true, otherwise return false.

\section{E.2.2.4 Testing and Programming}

The primary activity in this phase is programming or coding the individual units or modules [McDermid 93]. Work may also be carried out on producing test harnesses or stubs. The second activity is the testing of the program units after they have been programmed. The aim of unit testing is to check that a program unit matches the specification produced for it during $\mathrm{C}++$ Debug system design. Unit testing is a structural testing activity, the aim being to ensure some degree of test thoroughness with respect to 
some measure of structural coverage. A typical measure is that the test data generated should ensure that $100 \%$ of the statements in a unit are executed [McDermid 93]. Although this is a common metric, it is beginniging to be regarded as inadequate, and the better metric of $100 \%$ statement coverage and $85 \%$ branch coverage is being gradually adopted in industry. For example, Figure 92 shows the template of function used variable and its path. We must make sure that every path in the program is tested.

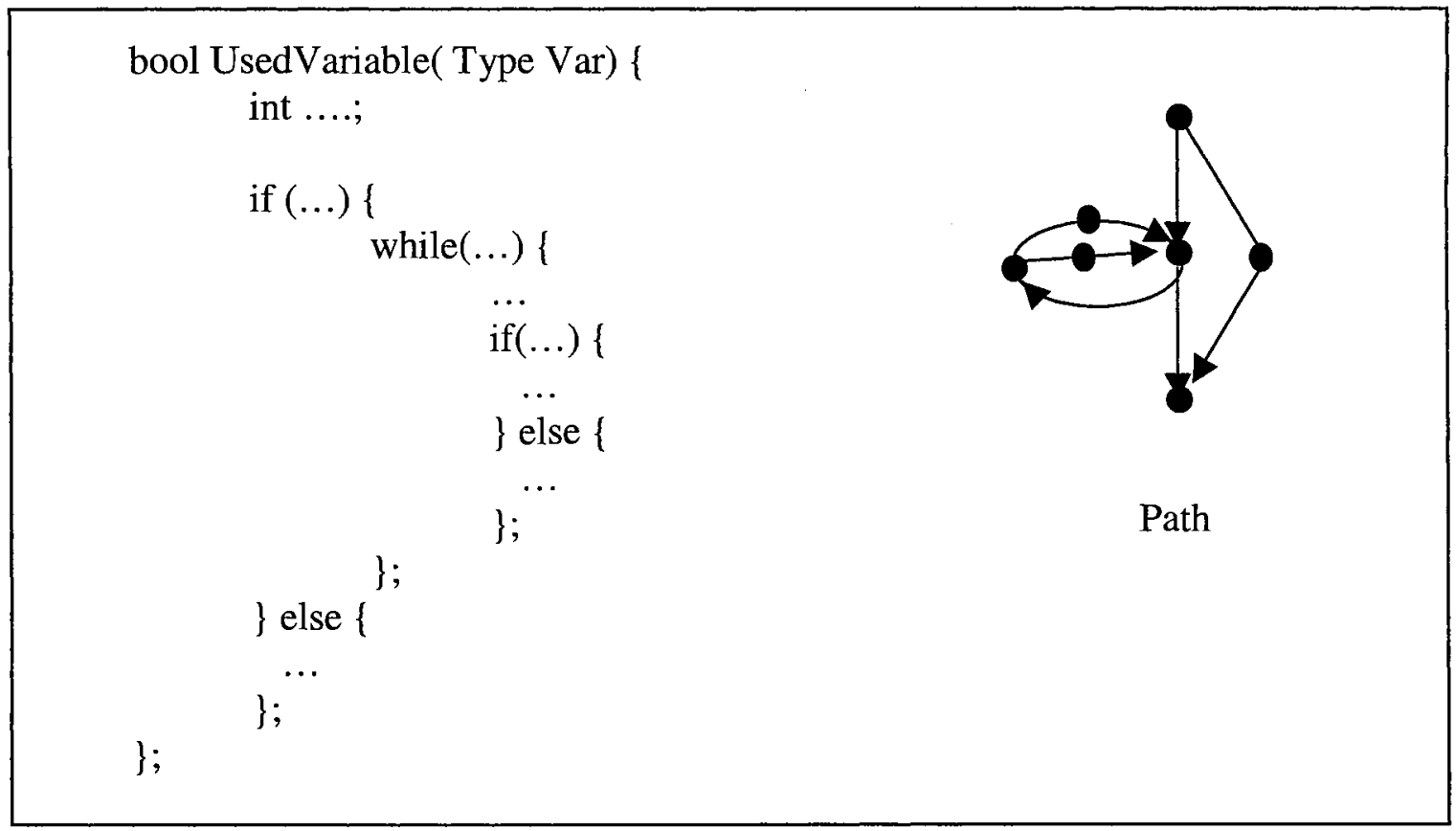

Figure 92. Part of function UsedVariable and its path

\section{E.2.2.5 Testing and Integration}

Testing during the integration phase will follow the plan set out in the system or architecture design [McDermid 93]. The primary aim of testing at this stage is to verify the design, but a subsidiary aim is to begin to verify the requirements. 
After coding and testing, individually, tested program unit are produced. These units are then progressively integrated according to the agreed strategy, e.g., top-down or bottom-up. A number of specific facets of the design are tested, leading up to the testing of the full design and requirements functions. For example, in $\mathrm{C}++$ Debug, module coupling and cohesion, as depicted in Figure 93, was tested.

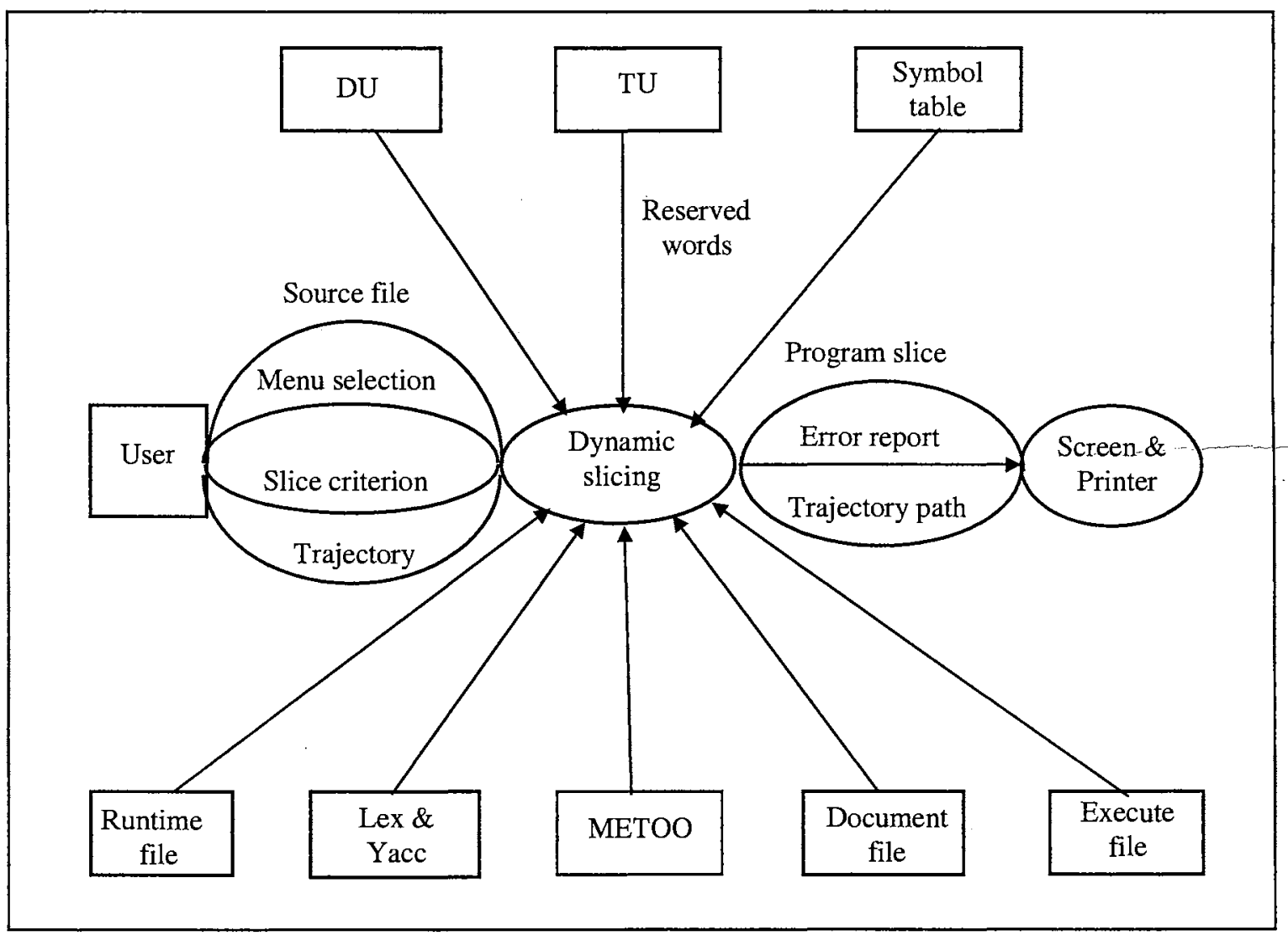

Figure 93. $\mathrm{C}++$ Debug block diagram

\section{E.2.2.6 System and Acceptance Testing}

In contrast to unit testing, system and acceptance testing are black-box activities [McDermid 93]. System testing is the process of executing the test procedures associated 
with the verification tests. Acceptance testing is the process of executing the test procedures associated with a subset of the verification requirements that are agreed on by both the customer and the developer as being an adequate representation of the user requirements. The major difference between system and acceptance testing is the fact that the former takes place in a simulated environment. For example, in C++Debug all modules in Figure 93 were tested as a system.

\section{E.2.2.7 Testing and Maintenance}

The last testing activity associated with the software life cycle is regression testing. This occurs during maintenance after a system has been modified [McDermid 93]. Therefore, this kind of test will be not applied to C++Debug. Regression testing is the execution of a series of tests to check that a modification, applied during maintenance, has not affected the code corresponding to those function of the system which should be unaffected by the maintenance modification that had been carried out.

\section{E.2.3 Testing Techniques}

The aim of this section is to show that the various techniques, which can be used to support the testing activities described in the previous section, were used to test C++Debug.

\section{E.2.3.1 Random Testing}

Random testing is a technique that is applicable for unit testing as a useful adjunct to other testing techniques [McDermid 93]. It involves identifying the input data space for a program unit and randomly generating test data from inside that space. In the case of $\mathrm{C}++$ Debug, a number of $\mathrm{C}++$ programs were used for testing. 


\section{E.2.3.2 Structural Testing}

Structural testing involves testing a software system so that some structural metric or a particular path is traversed [McDermid 93]. In the case of C++Debug, every path of the control statements was tested.

\section{E.2.3.3 Assertion Testing}

An assertion is a predicate that relates the value of variables in a program and describes a condition that must be true during the execution of a program unit or a program [McDermid 93]. In the case of $\mathrm{C}++$ Debug, for example,

$$
\text { Dice_A_B }==\text { Slice_A \& Slice_B }
$$

represents a relation that must hold between the three variables used in the condition. Such conditions can be inserted by hand or by mean of software tools.

\section{E.2.3.4 Grammar-Based Testing}

Grammar-based testing is based on describing the data set to be used in a test by means of some grammar formalism [McDermid 93]. In the case of C++Debug, for example from Figure 93, the C++ grammars, which implemented in the "Lex \& Yacc" block, were tested.

\section{E.2.3.5 Functional Testing}

Functional testing involves checking the functions of a computer system by means of examining either the system specification or the system design [McDermid 93]. In the case of $\mathrm{C}++$ Debug, for example, all functions in Appendices $\mathrm{C}$ and $\mathrm{D}$ were tested. 


\section{E.3 Evaluation}

\section{E.3.1 Introduction}

C++Debug was evaluated based on Lyle [Lyle 84] and Gallagher's [Gallagher 90] approach by training several Computer Sciences graduate students at Oklahoma State University in its operation and by collecting data on how the students used $\mathrm{C}++$ Debug to locate faults in $\mathrm{C}++$ programs. The main objective of the evaluation was how can $\mathrm{C}++$ Debug be used to enhance the debugging process and localize errors.

\section{E.3.2 Evaluation Procedure}

The debugging process was studied by allowing each student to debug one program with and without $\mathrm{C}++$ Debug. There were four steps as listed below.

\section{E.3.2.1 Step I: Familiarization}

Let each student answer a questionnaire covering background information (see Subsection E.3.2.4), read an overview of the evaluation, and finally read the manual on how to use $\mathrm{C}++$ Debug (see Appendix B).

\section{E.3.2.2 Step II: First Treatment}

Let each student debug $\mathrm{C}++$ programs without using the $\mathrm{C}++$ Debug tool. Each student can use other tools such as DBX, GDB, etc.

\section{E.3.2.3 Step III: Second Treatment}

In this step, the $\mathrm{C}++$ programs in step II were debugged by using the $\mathrm{C}++$ Debug tool. 


\section{E.3.2.4 Step IV: Subject Remarks}

All information from Step I, Step II, and Step III were collected and analyzed based on Lyle's [Lyle 84] approach to find out:

1. Is $\mathrm{C}++$ Debug useful?

2. Are there some negative and positive comments?

3. What do they like about $\mathrm{C}++$ Debug?

4. What don't they like about C++Debug?

The students involved in the evaluation of $\mathrm{C}++$ Debug were asked to fill out a questionnaire based on Lyle's [Lyle 84] approach as follows.

Questionnaire

(1) How long have you been programming (Years/Months)?

(2) How many CS, (Computer Science), classes in your BS/BA?

(3) How many CS classes taken so far in grad school?

(4) How many other CS classes have you taken?

(5) Which programming languages are you familiar with? Familiar means you used the language for at least a semester's work.

(6) On a scale from 0 to 10 , how familiar are you with $\mathrm{C}++$ ? where $0=\mathrm{I}^{\prime}$ ve never used $\mathrm{C}++$ $2=\mathrm{I}$ know some $\mathrm{C}++$

$5=\mathrm{I}$ know $\mathrm{C}++$ about average

$7=\mathrm{I}$ am comfortable with $\mathrm{C}++$

$10=\mathrm{I}$ know $\mathrm{C}++$ well

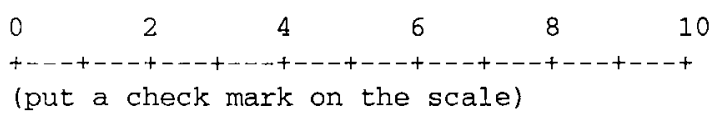

(7) On the same scale from 0 to 10 , how familiar are you with the VI or EMACS text editor?

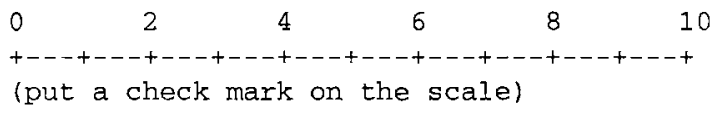

(8) Do you know about program slicing? 
The subjects involved in the evaluation of $\mathrm{C}++$ Debug were ten graduate students at the Computer Science Department of Oklahoma State University. The student responses to the questions are summarized in Table IV and V. The number of changes made to the tested programs by each student, and the number of slices each student computed are shown in Table VI. And finally, edit times, compile times, and execution times are presented in Tables VII and VIII.

\begin{tabular}{|l|c|c|c|c|c|c|}
\hline \multicolumn{7}{|c|}{ TABLE IV } \\
\hline \multicolumn{1}{|c|}{ Variable } & $\mathrm{N}$ & mean & sd & min & max & median \\
\hline time_programming & 10 & 8.1 & 3.0 & 3.0 & 13.0 & 8.5 \\
n_bs_classes & 10 & 7.4 & 5.8 & 0.0 & 15.0 & 8.5 \\
n_ms_classes & 10 & 10.2 & 2.5 & 6.0 & 15.0 & 9.5 \\
n_other_classes & 10 & 1.6 & 1.8 & 0.0 & 4.0 & 1.0 \\
n_languages & 10 & 7.9 & 2.2 & 4.0 & 12.0 & 8.0 \\
skill_C++ & 10 & 7.1 & 2.5 & 2.0 & 10.0 & 8.0 \\
skill_vi_or_emacs & 10 & 7.8 & 2.9 & 1.0 & 10.0 & 9.0 \\
\hline
\end{tabular}

\begin{tabular}{|l|c|}
\hline \multicolumn{2}{|c|}{ TABLE V } \\
\hline \multicolumn{2}{|c|}{ LANGUAGE FREQUENCY } \\
\hline Language & Number of Subjects \\
\hline Assembler & 3 \\
C & 9 \\
C++ & 7 \\
Java & 6 \\
Lisp & 2 \\
Pascal & 3 \\
\hline
\end{tabular}




\begin{tabular}{|c|c|c|}
\hline \multicolumn{3}{|c|}{ TABLE VI } \\
\hline \multicolumn{3}{|c|}{ SLICES AND PROGRAM CHANGES } \\
\hline Subject & Slices & Changes \\
\hline 1 & 4 & 3 \\
2 & $*$ & 2 \\
3 & $*$ & 3 \\
4 & 1 & 3 \\
5 & $*$ & 2 \\
6 & $*$ & 3 \\
7 & 4 & 3 \\
8 & 8 & 7 \\
9 & 3 & 5 \\
10 & $*$ & 3 \\
\hline
\end{tabular}

* not slicing

TABLE VII

TIME MEASURES FOR DEBUGGING BY USING THE TOOL

\begin{tabular}{|l|c|c|c|c|c|c|}
\hline & $\mathrm{N}$ & mean & sd & $\min$ & $\max$ & median \\
\hline Edit user time & 10 & 832 & 397 & 352 & 1177 & 782 \\
Edit system time & 10 & 437 & 184 & 194 & 486 & 412 \\
\hline Compile user time & 10 & 15490 & 2822 & 11882 & 16957 & 14510 \\
Compile system time & 10 & 4664 & 842 & 3872 & 5543 & 4602 \\
\hline Execute user time & 10 & 580 & 223 & 391 & 774 & 460 \\
Execute system time & 10 & 845 & 212 & 618 & 1021 & 757 \\
\hline
\end{tabular}

\section{E.3.3 Comments on $\mathrm{C}++$ Debug}

Seven of the ten subjects reported that in the slicing mode C++Debug was very useful. In the dicing mode, four subjects reported that $\mathrm{C}++$ Debug can help them to locate errors in a program. Five subjects felt surprised that $\mathrm{C}++$ Debug could eliminate 
irrelevant statements. Three subjects said that in the $-t$ mode the trajectory path generated by $\mathrm{C}++$ Debug worked like the cpptrace tool in $\mathrm{C}$, in an effective and useful manner.

\begin{tabular}{|l|c|c|c|c|c|c|}
\hline \multicolumn{7}{|c|}{ TABLE VIII } \\
\hline \multicolumn{1}{|c|}{ TIME MEASURES FOR DEBUGGING WITHOUT USING THE TOOL } \\
\hline Edit user time & $\mathrm{N}$ & mean & sd & min & max & median \\
Edit system time & 10 & 1224 & 1012 & 371 & 2501 & 902 \\
\hline Compile user time & 10 & 713 & 633 & 286 & 1522 & 411 \\
Compile system time & 10 & 12501 & 492 & 11903 & 12833 & 12532 \\
\hline Execute user time & 10 & 3962 & 557 & 3255 & 4482 & 3921 \\
Execute system time & 10 & 588 & 113 & 464 & 621 & 521 \\
\hline
\end{tabular}

On the negative side, one subject felt that $\mathrm{C}++$ Debug was not more powerful than other debugging tools like GDB. Two subjects mentioned that the dicing process is quite complicated because of the process of selecting the appropriate slicing criteria (variables and positions for dicing). One subject mentioned that in the $-\mathrm{g}$ mode, $\mathrm{C}++$ Debug generated derivation tree that were too long, and that it was difficult to understand all of them. 


\section{APPENDIX F \\ SAMPLE PROGRAMS USED FOR THE COMPUTATION \\ OF SLICING-BASED METRICS}

The sample programs shown below were used for the computation of slicing-

based metrics (see Section 4.8 for more detail). In order to compare the output obtained from $\mathrm{C}++$ Debug (which is based on dynamic slicing) with C-Sdicer (which is based on static slicing), the test programs must be the same as the ones used in Nanja's study in testing C-Sdicer [Nanja 90]. These test programs are provided bellow.

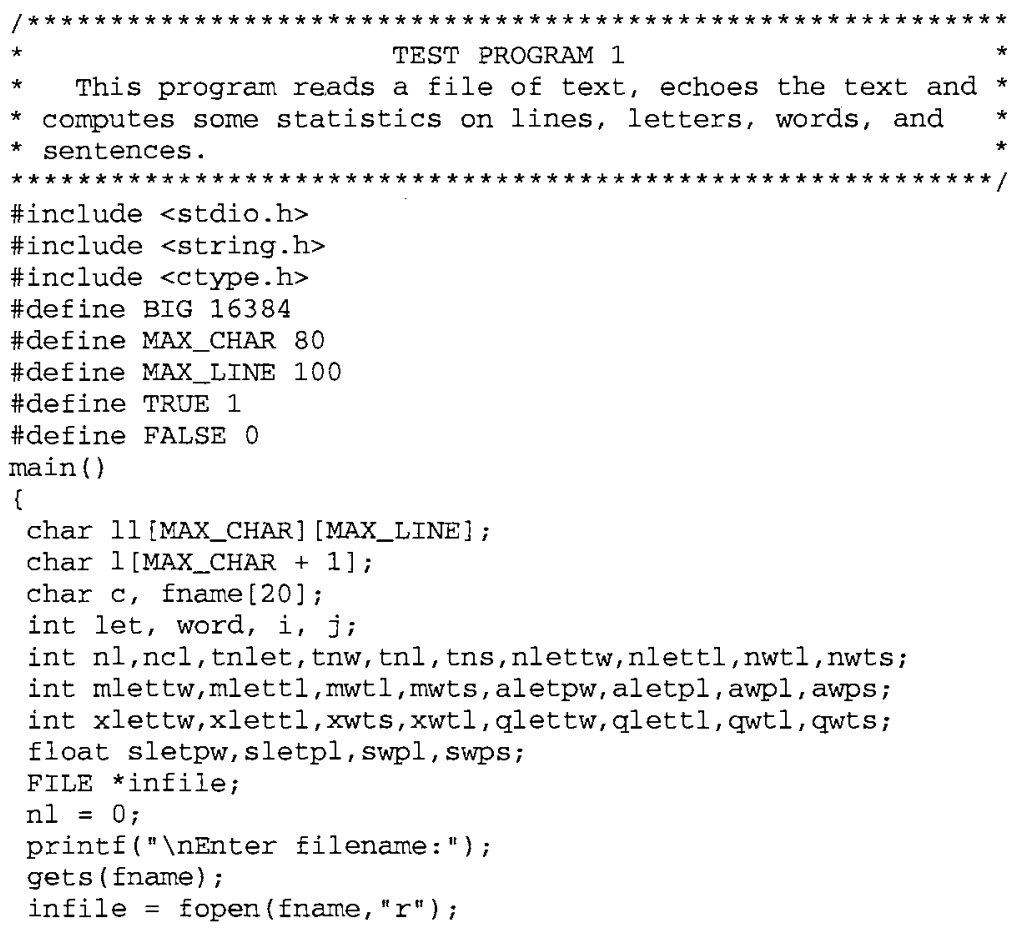




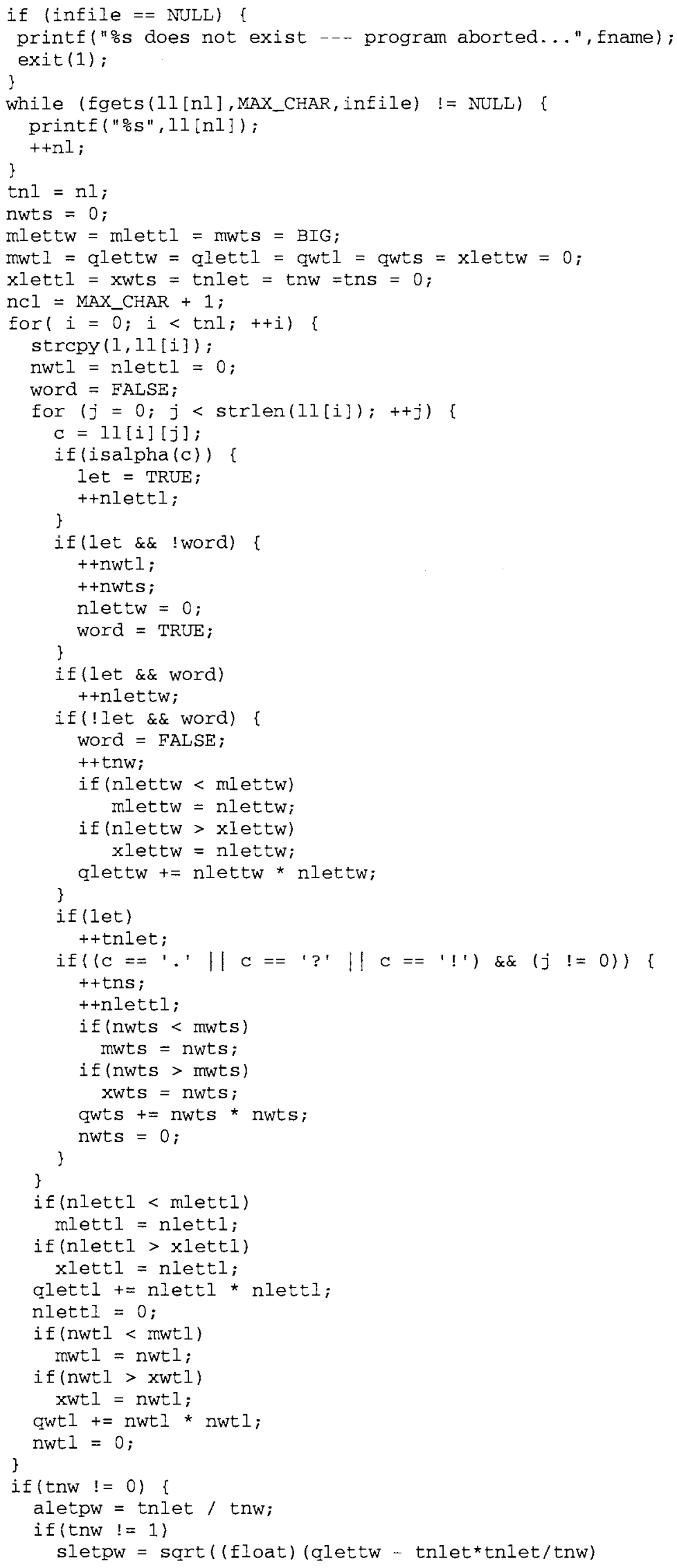




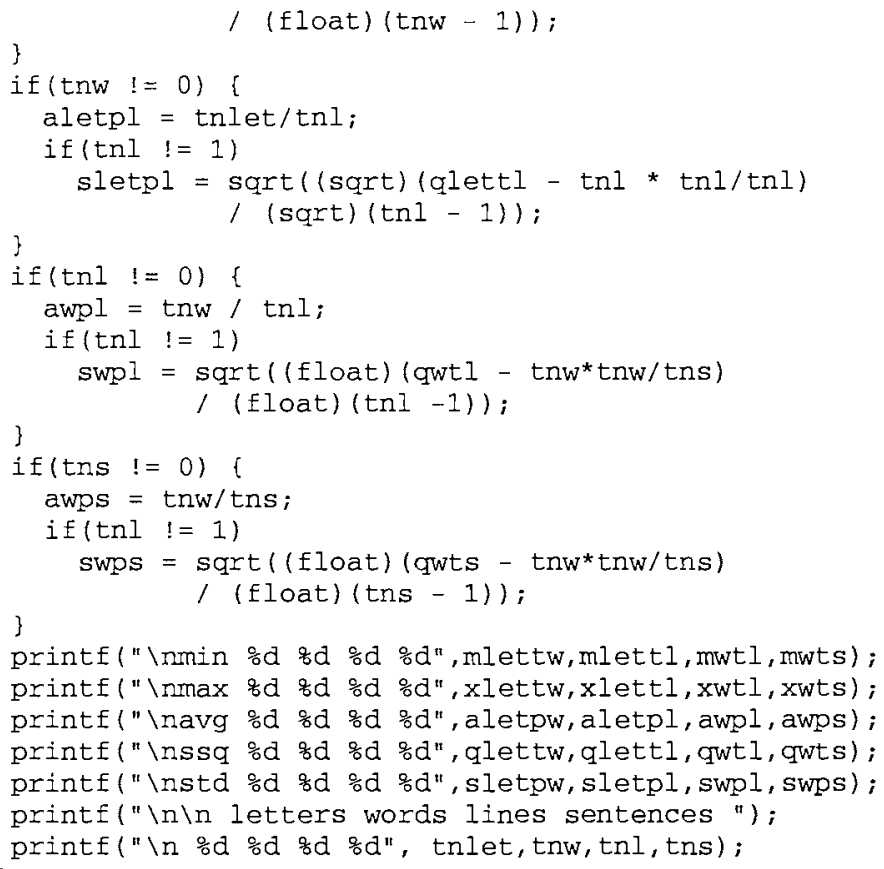

Program 1 (P1) Source: [Nanja 90]

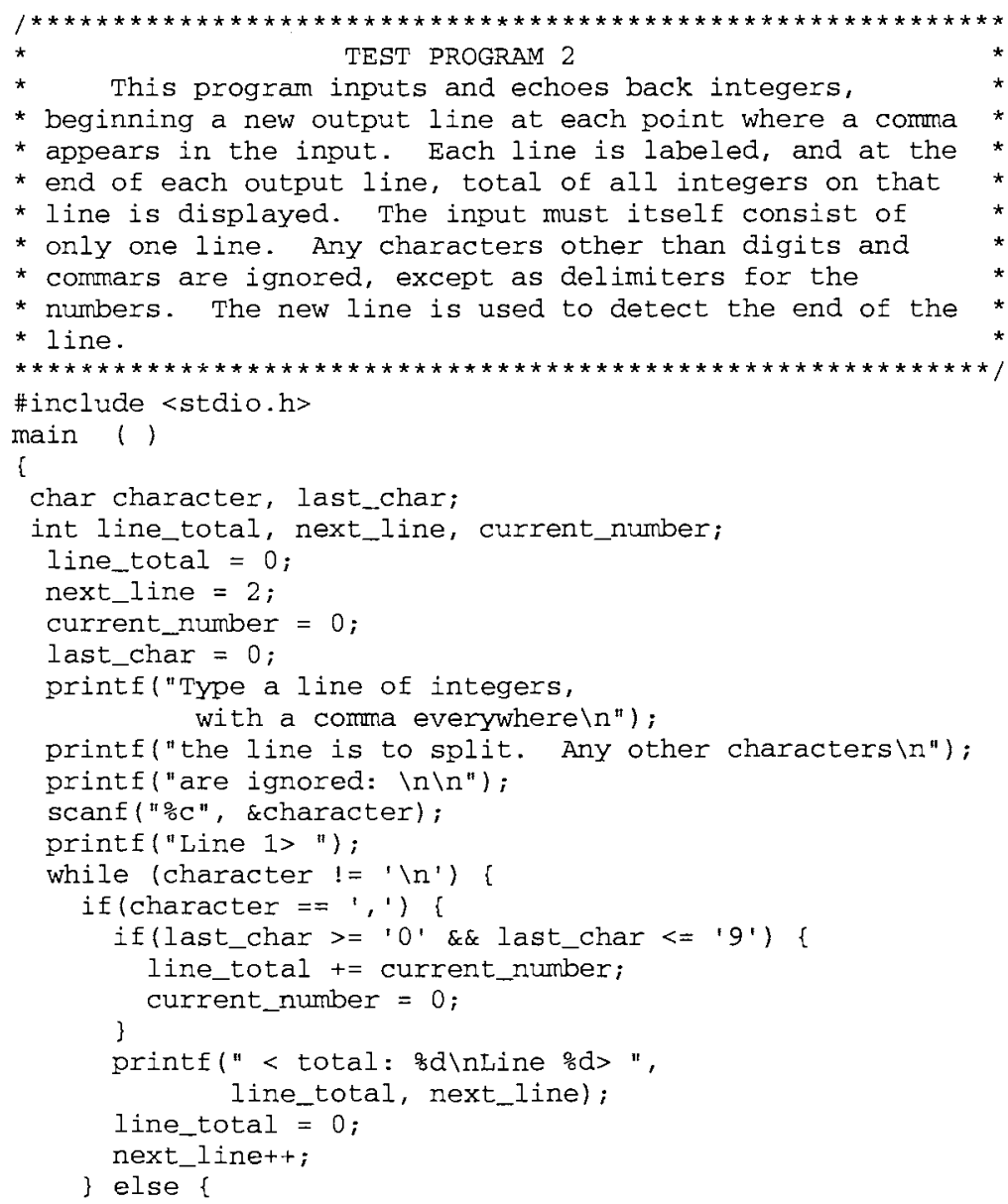




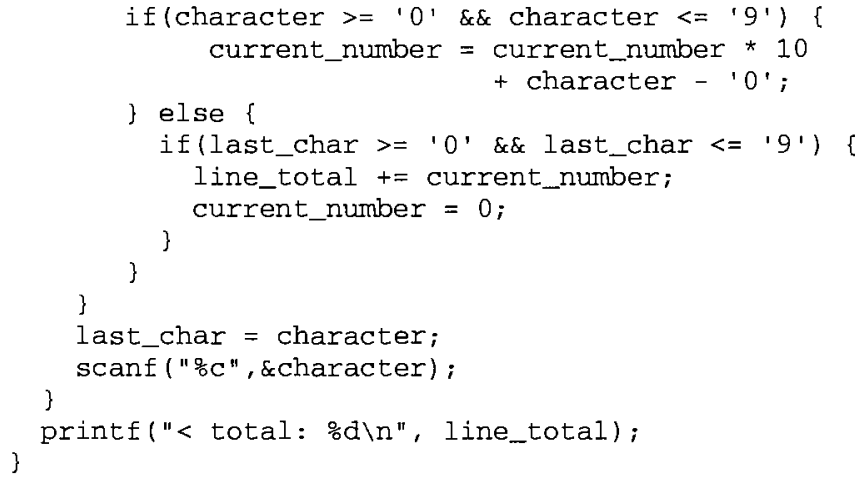

Program 2 (P2) Source: [Nanja 90]

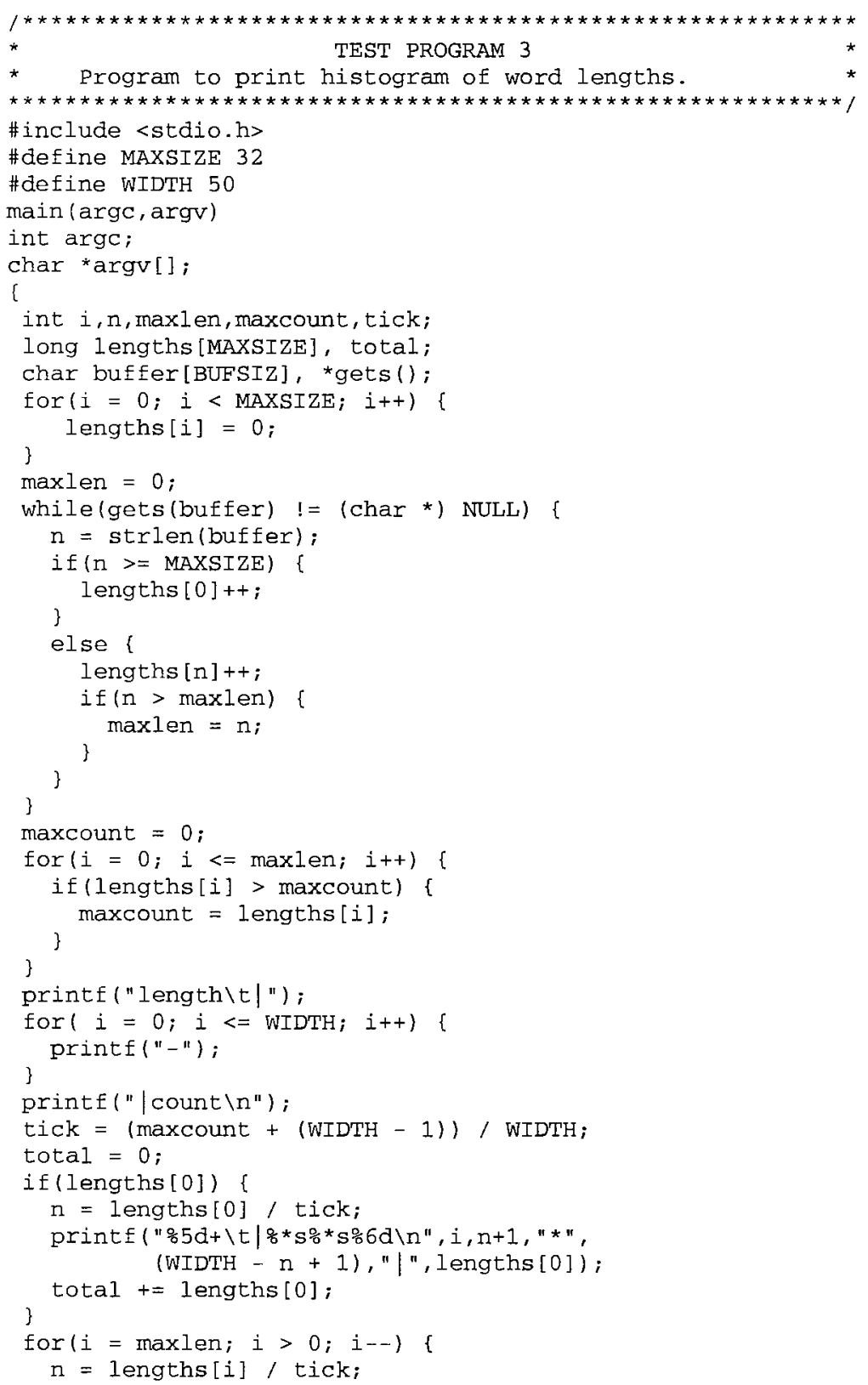




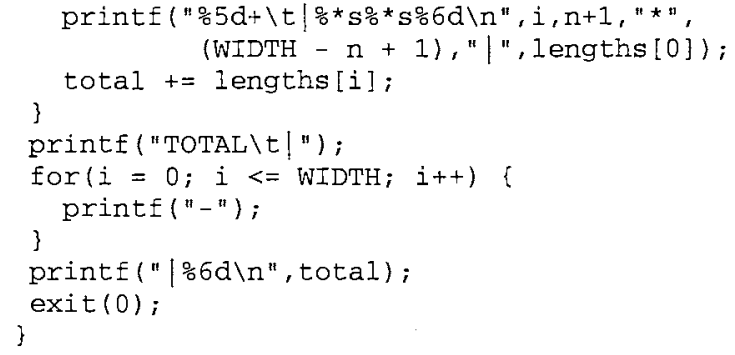

Program 3 (P3) Source: [Nanja 90]

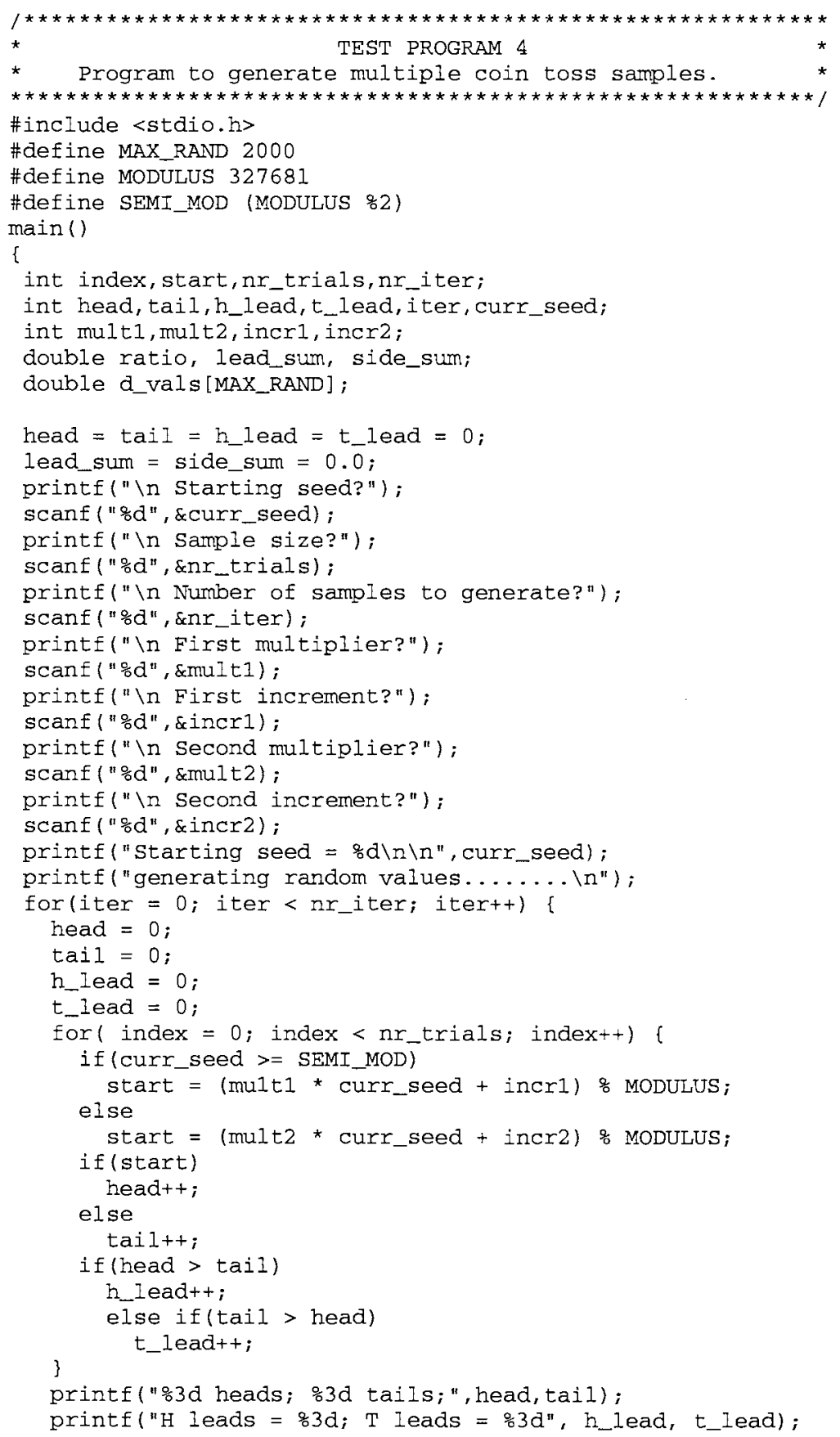




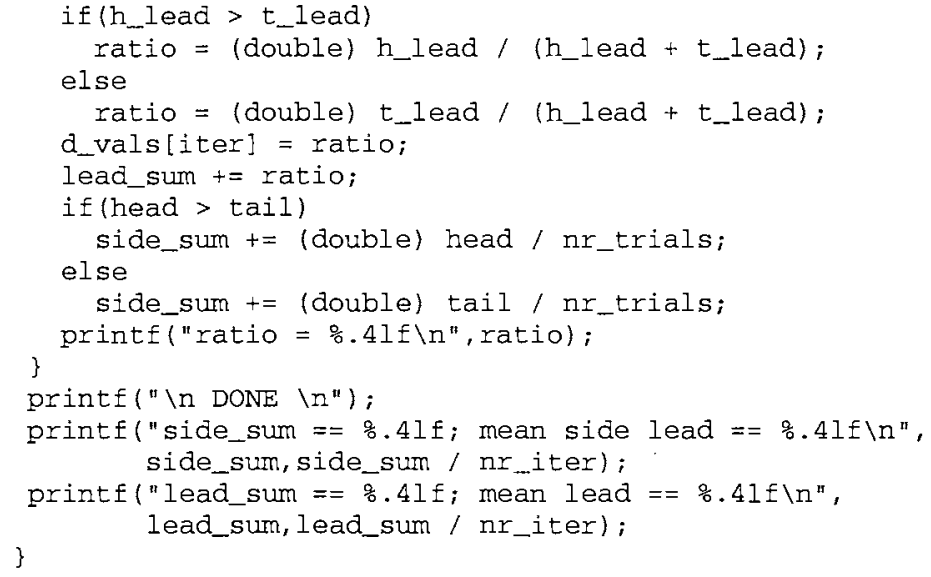

Program 4 (P4) Source: [Nanja 90]

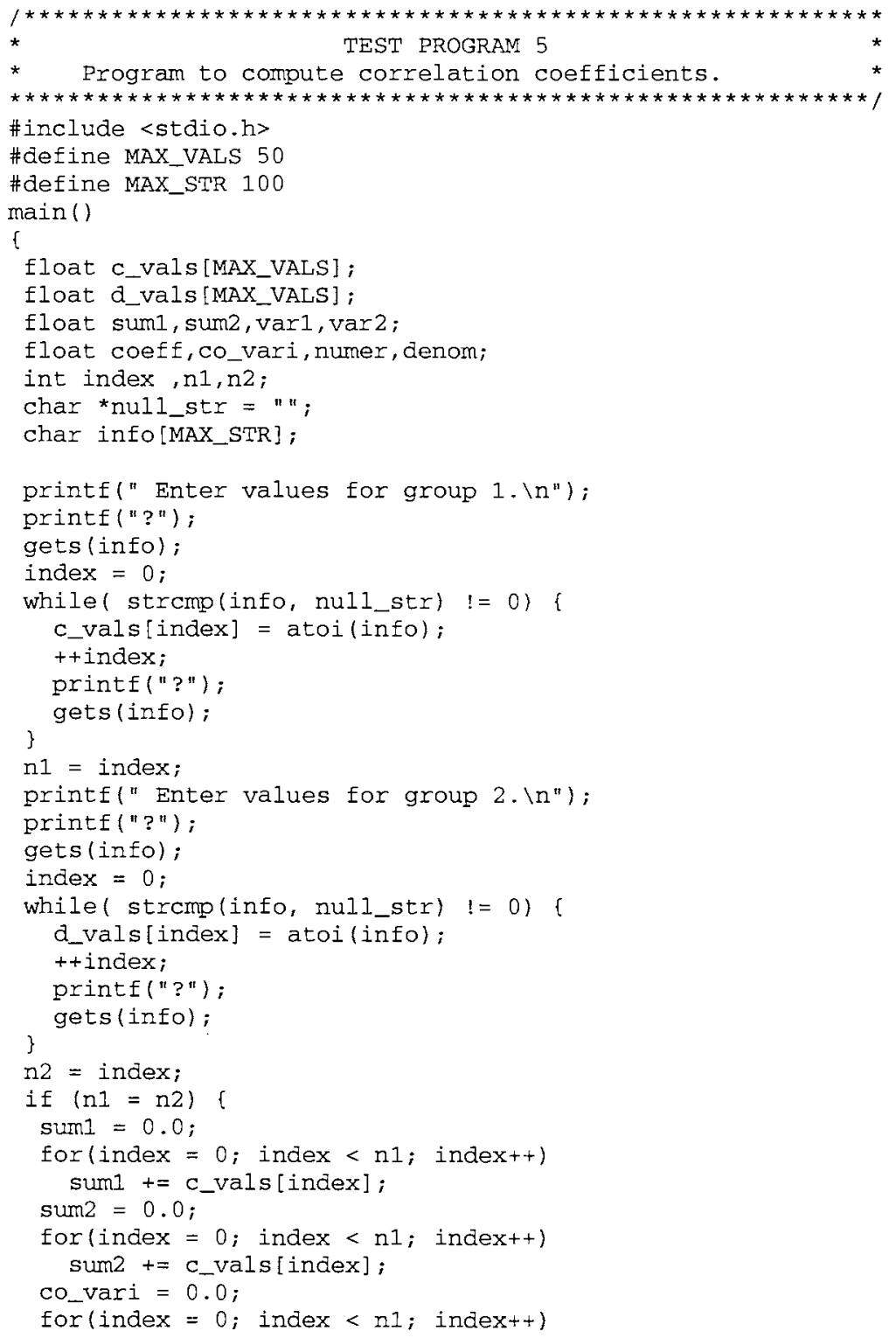


Co_vari $+=$ c_vals[index] * d_vals[index];

numer $=$ co_vari $-($ sum1 * sum2 $)$;

$\operatorname{var} 1=0.0$;

for (index $=0$; index $<\mathrm{n} 1$; index ++ )

varl $t=$ c_vals[index] * c_vals[index];

for (index $=0$; index $<\mathrm{n} 1$; index ++ )

var2 $+=$ d_vals[index] * d_vals[index]

denom $=(\operatorname{var} 1-\operatorname{sum} 1 * \operatorname{sum} 2) *(\operatorname{var} 2-\operatorname{sun} 2 * \operatorname{sum} 2) ;$

denom $=\operatorname{sqrt}($ denom $) ;$

if (denom $!=0$ )

coeff = numer / denom;

printf ("r $==\frac{8}{8} 7.311 \pm \backslash n "$, coeff);

else

printe("Arrays must be the same size. $\backslash n ")$;

Program 5 (P5) Source: [Nanja 90] 


\section{APPENDIX G}

\section{SAMPLE C++DEBUG SOURCE CODE LISTING}

$\mathrm{C}++$ Debug is comprised of 24 files. The following files contain code written in the $\mathrm{C}++$ programming language. Some sample code segments are shown as well.

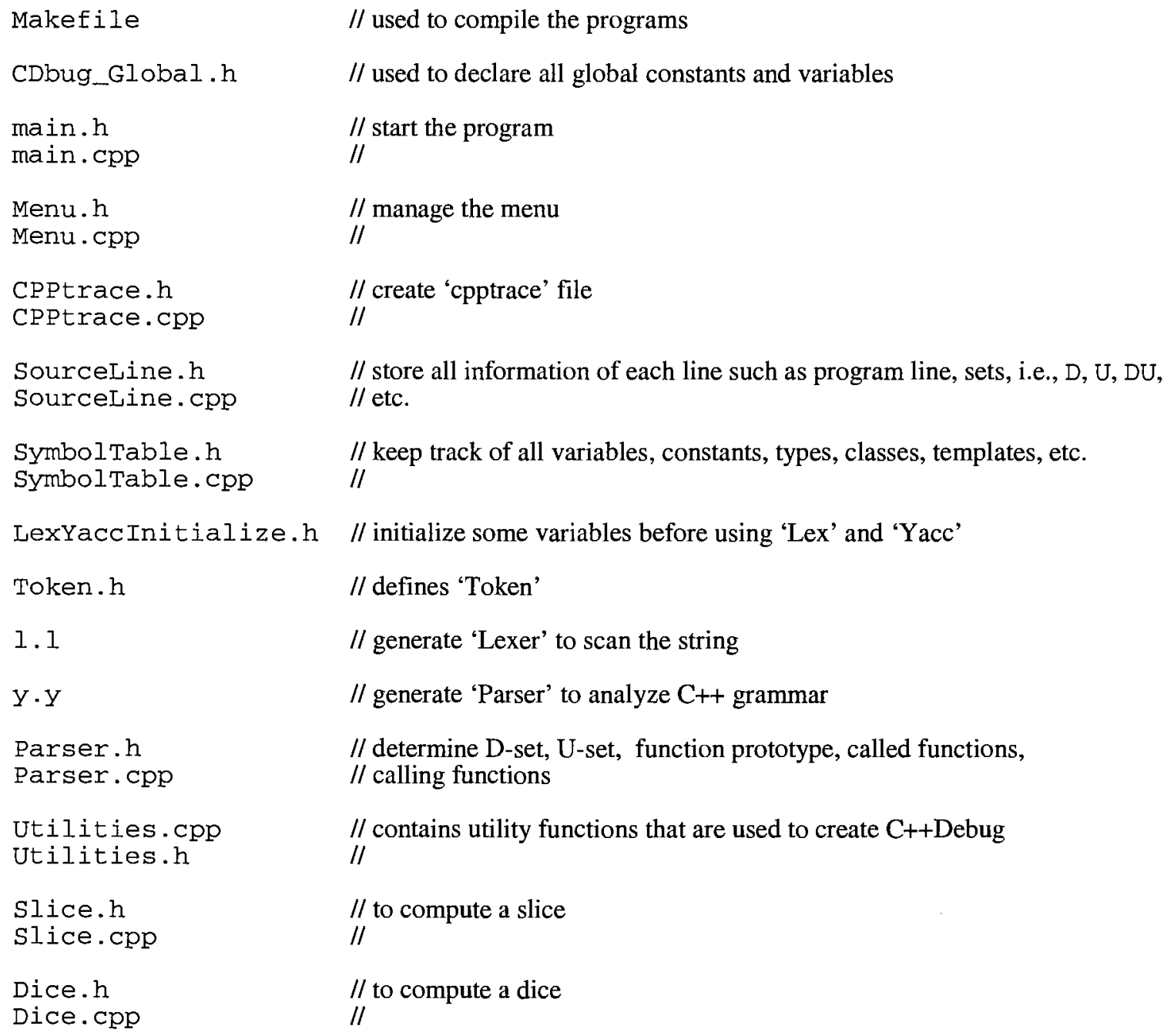




\section{C++DEBUG}

C++Debug is a slicing and dicing based debugging tool for ANSI C++ that

* runs under the UNIX or Linux operating system. It has been designed in a way *

* to provide ease and convenience on the part of the user. Using C++Debug,

* the user can interact with the computer in locating errors in a program. For *

* convenience of the user, menu is introduced that allows the user to

* select any one of the available functions.

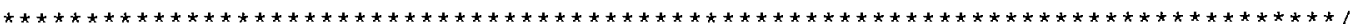

\#include "main.h"

main(int argc, char *argv[]) [

FLAGINFO = false; $/ /$ Do not display C++Debug information

// Global initialize

MainInitialize();

CDbugMenu Menu;

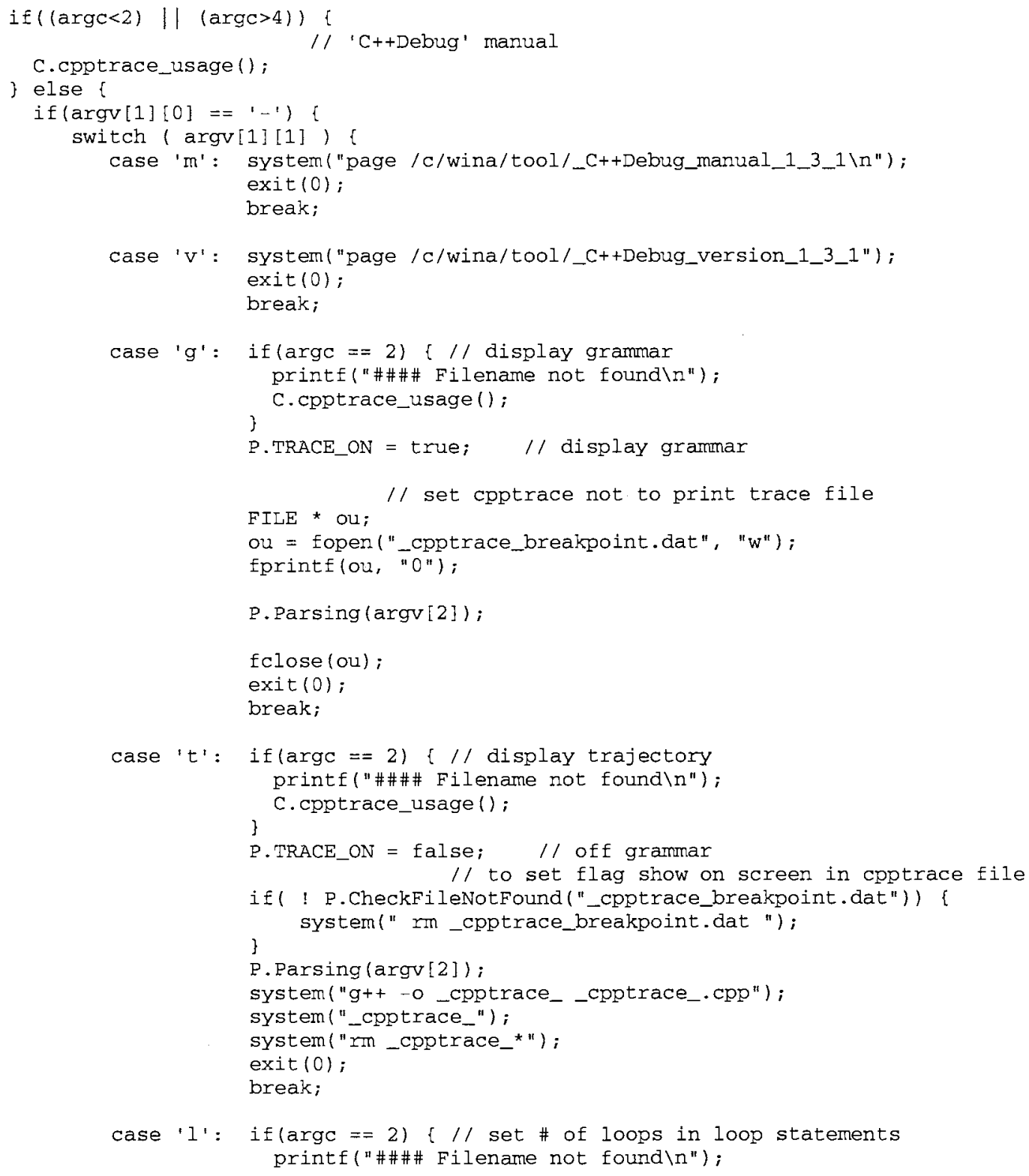




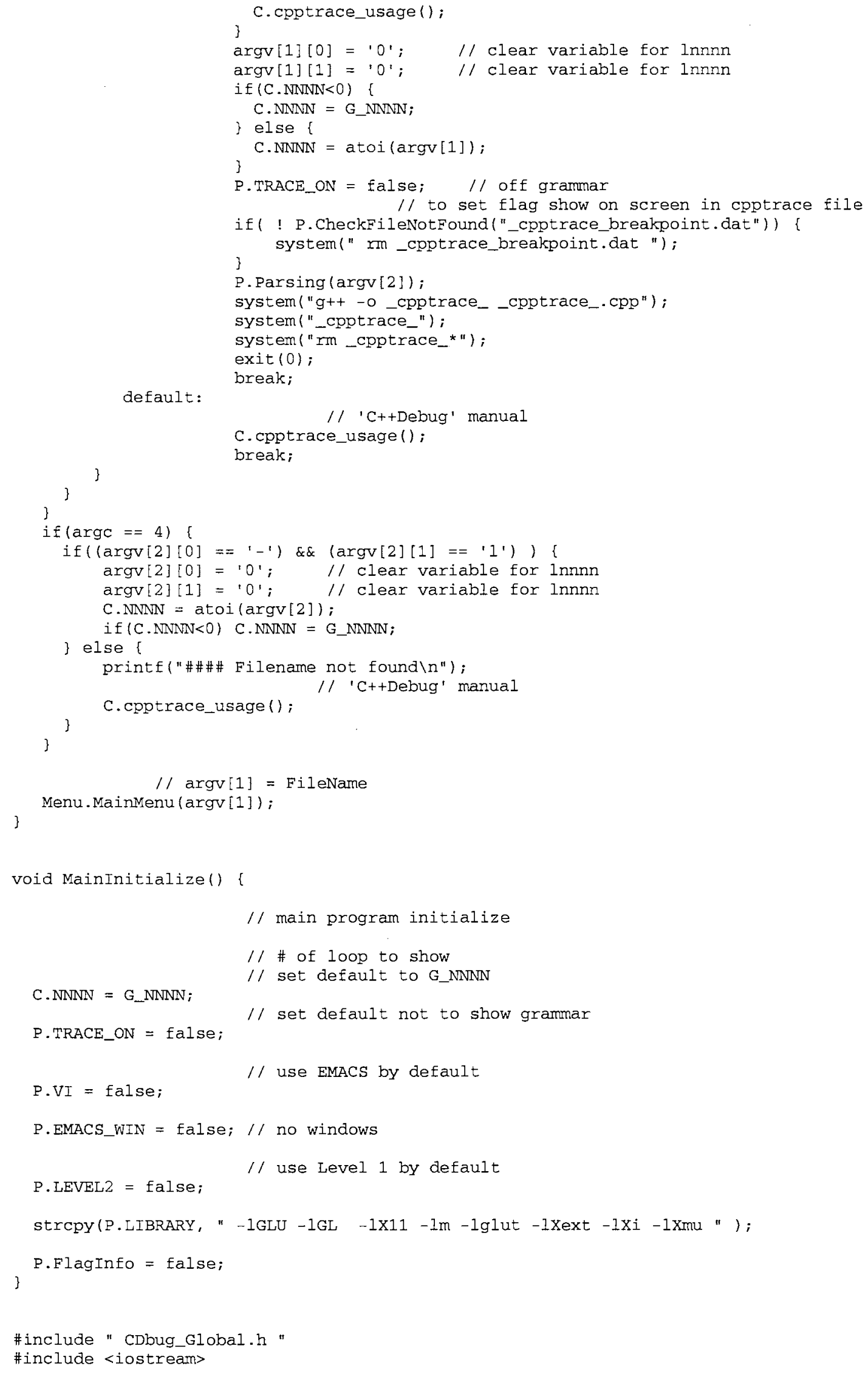




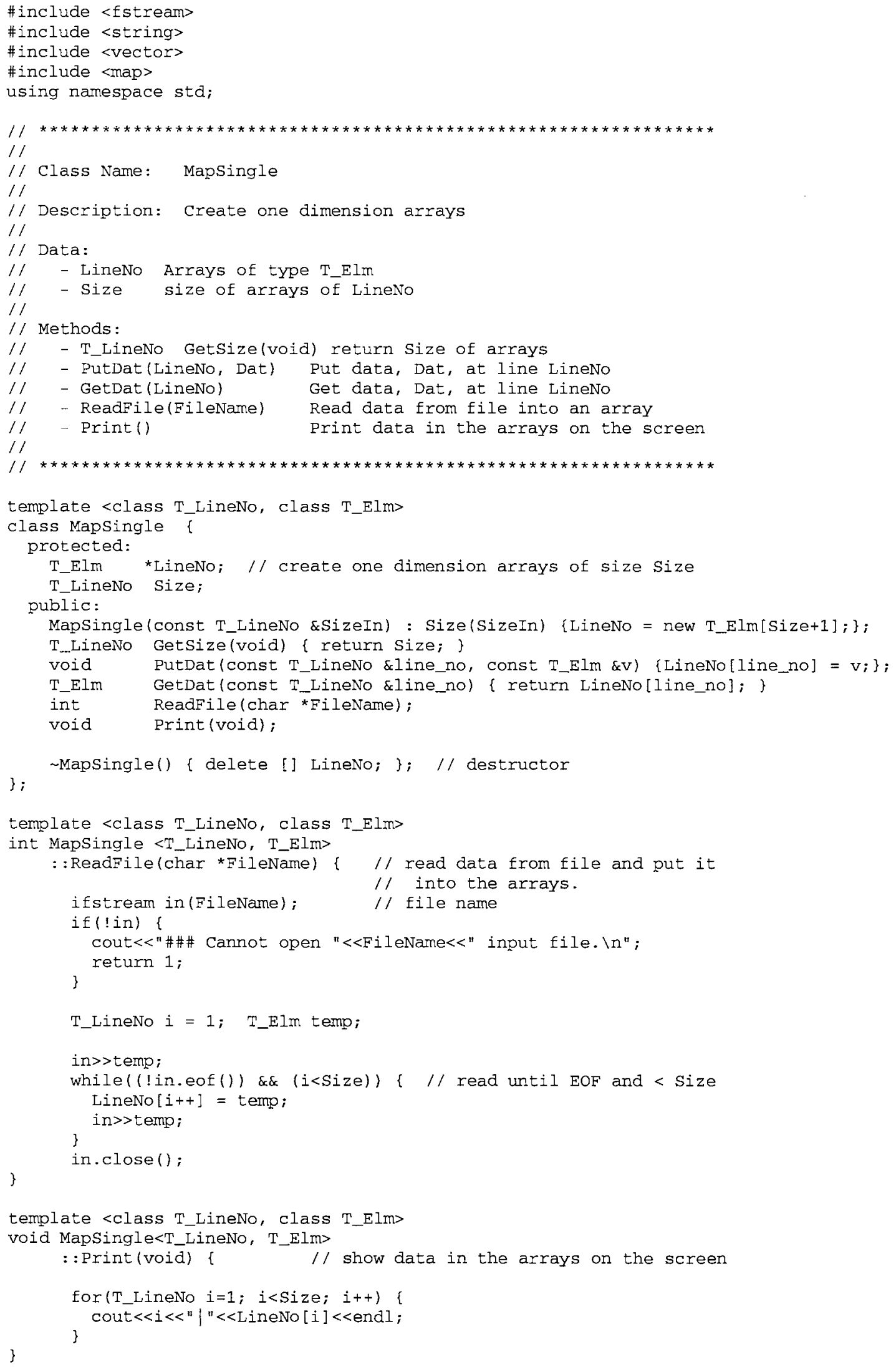




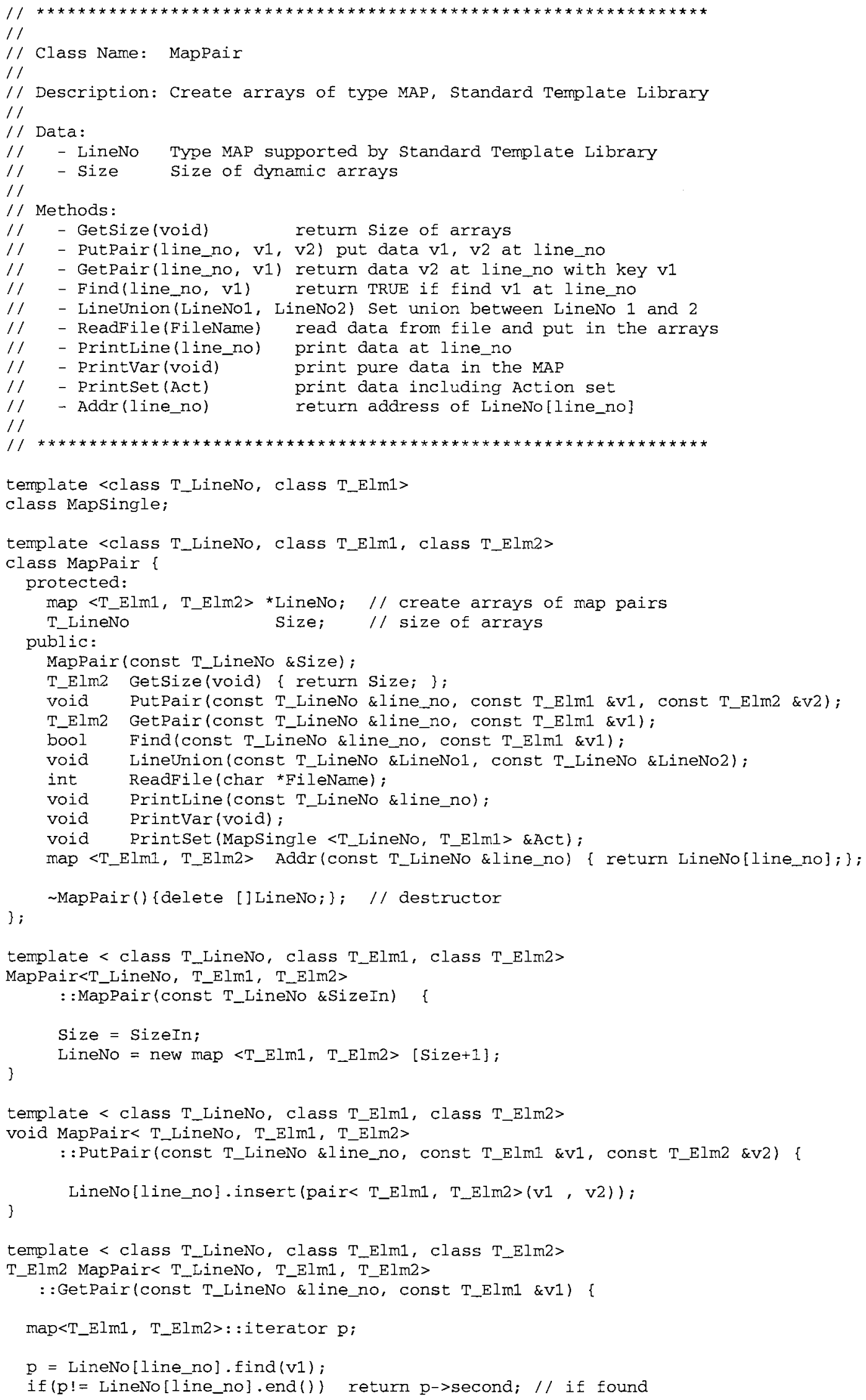


template < class T_LineNo, class T_Elm1, class T_Elm2> bool MapPair< T_LineNo, T_ElmI, T_Elm2>

: :Find(const T_LineNo \&line_no, const T_ElmI \&v1) \{

map<T_Elm1, T_Elm2>: : iterator p;

$\mathrm{p}=$ LineNo[line_no].find $(\mathrm{v} 1)$;

if $(p !=$ LineNo[line_nol.end()) return true; // if found

else return false;

$/ /$ if cannot find

\}

template < class T_LineNo, class T_Elm1, class T_Elm2>

void MapPair<T_LineNo, T_Elm1, T_Elm2>

:: LineUnion (const $\bar{T}_{\text {_LineNo }}$ \&LineNo1, const T_LineNo \&LineNo2) ,

map<T_Elm1, T_Elm2>: : iterator $\mathrm{p} ; / /$ set Union between two lines

$p=$ LineNo [LineNo2] . begin();

$/ /$ and store in the first line

while (p $!=$ LineNo[LineNo2]. end ()) \{

PutPair(LineNol, p->first, $p->$ second);

$p++;$

\}

template < class T_LineNo, class T_Elm1, class T_Elm2>

void Mappair< T_LineNo, T_Elm1, T_Elm2>

: :PrintLine(const T_LineNo \&line_no) \{

map<T_Elml, T_Elm2>: :iterator $p ; / /$ print data at line_no

$\mathrm{p}=$ LineNo[1ine_no].begin();

cout $<$ "Line No. "<<line_no $<<"$ : ;

while (p $!=$ LineNo[line_no . end ()) \{

cout $<<" \mid "<<$ p->first $<<", "<<$ p- second $<<" \mid$ ";

\} $p++$;

cout $<<$ endl

\}

template < class T_LineNo, class T_Elm1, class T_Elm2>

void MapPair< T_LineNo, T_Elm1, T_Elm2>

: : Printvar(void) \{

for (T_LineNo $i=0 ; i<$ Size; $i++) / /$ print all data

PrintLine (i);

\}

cout<endl;

template <class T_LineNo, class T_Elm1, class T_Elm2>

int MapPair<T_LinêNo, T_Elm1, T_Elm2>

: :ReadFile(char *FileName)

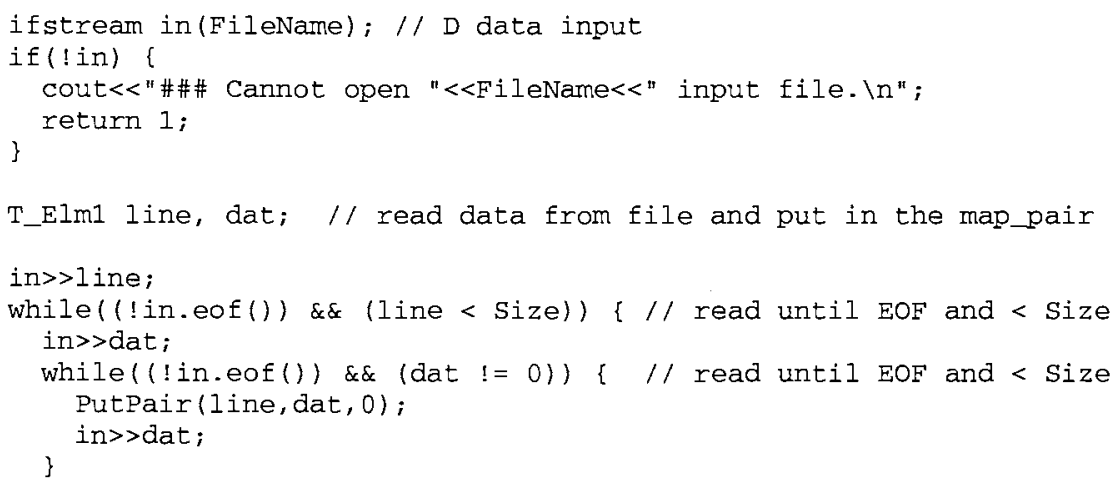




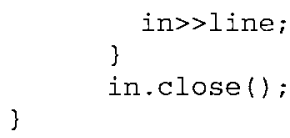




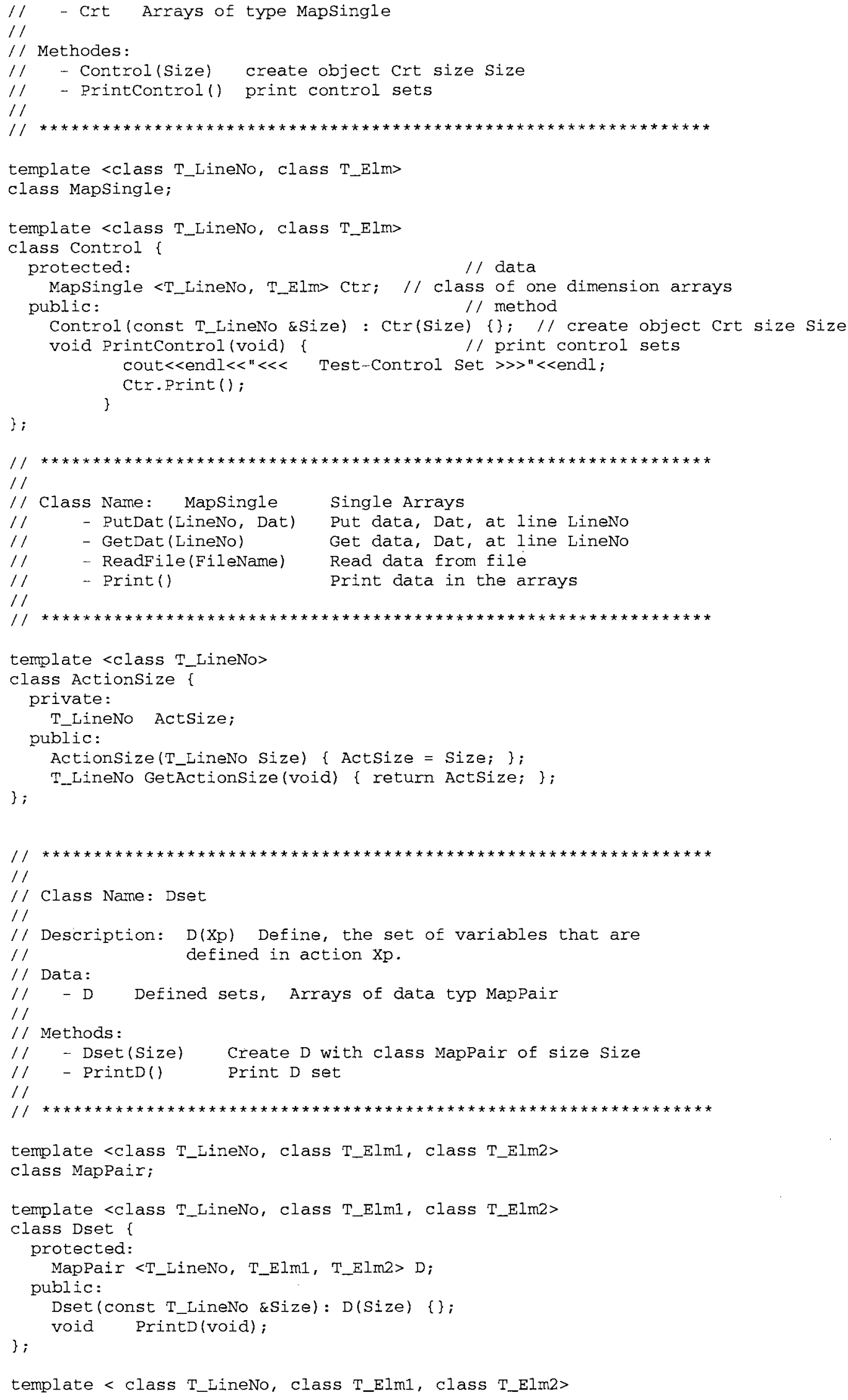




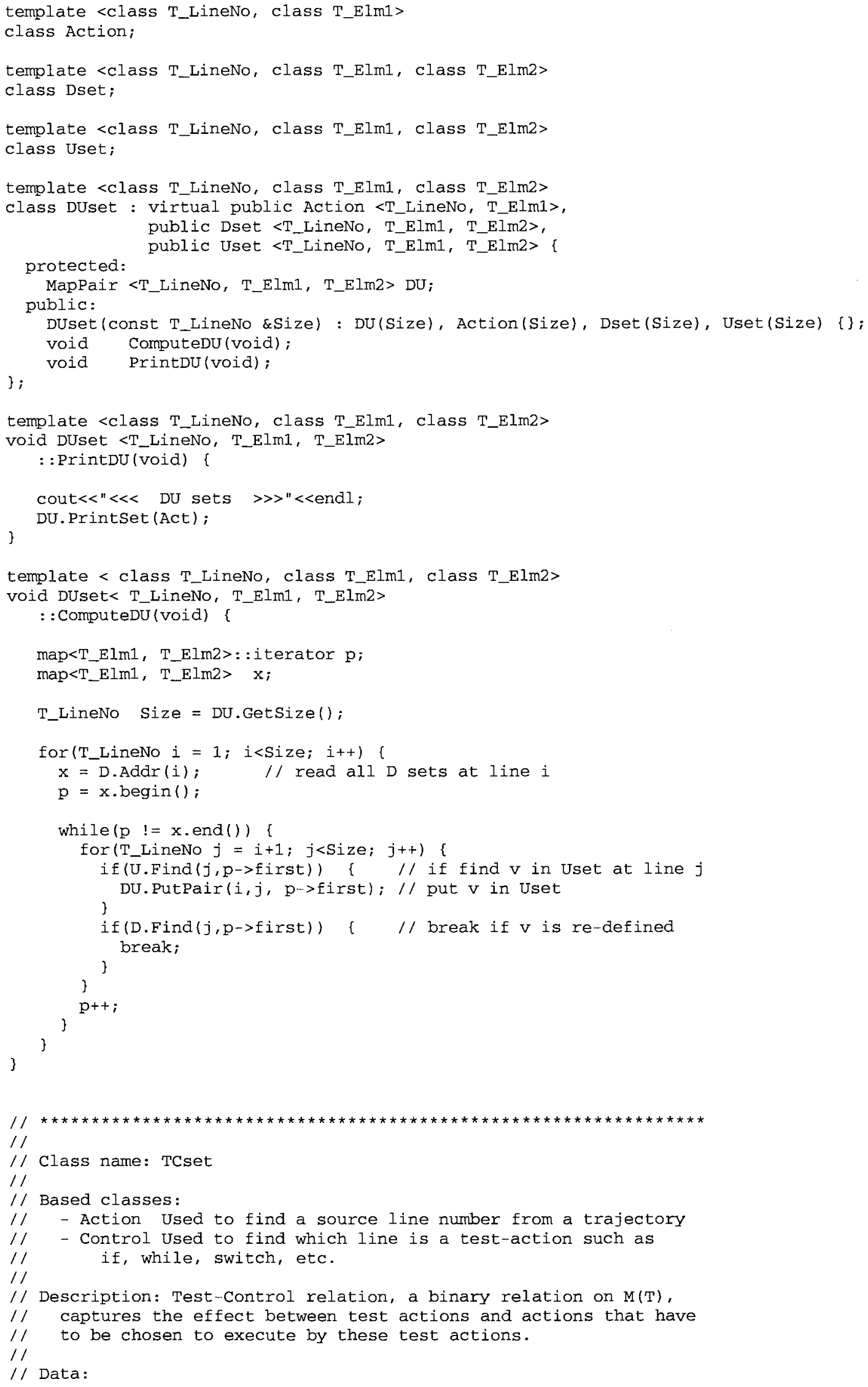




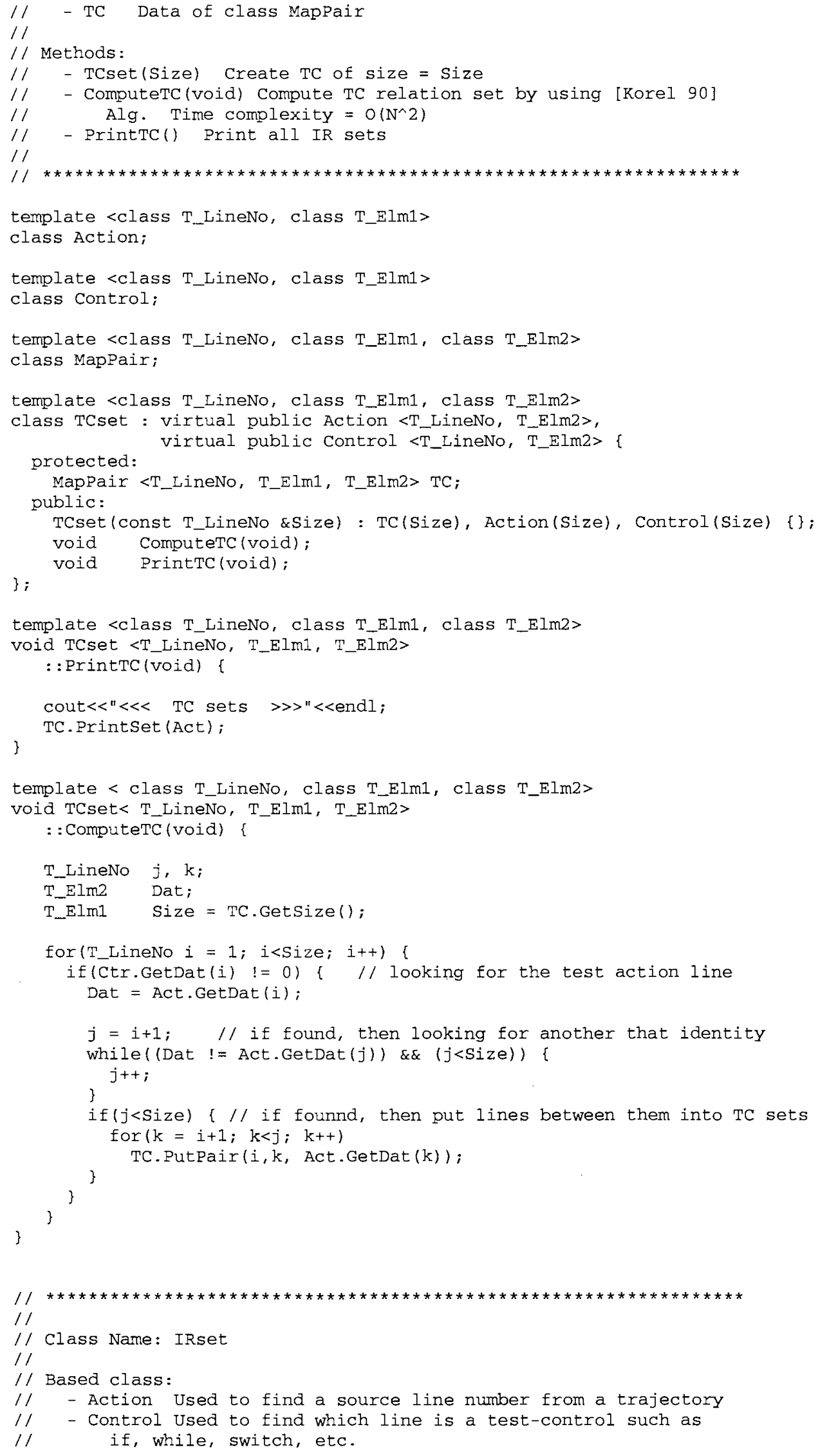




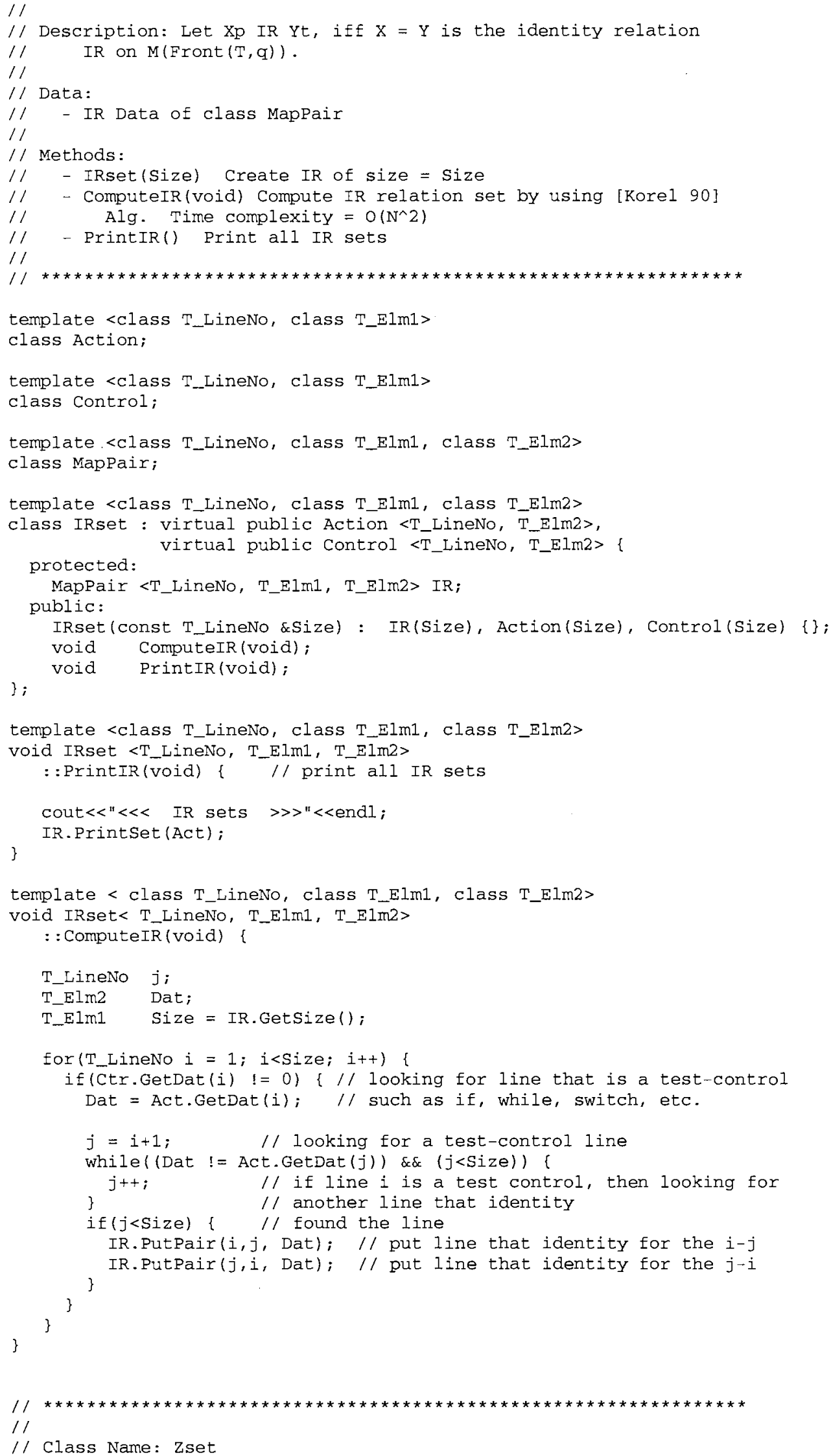


$1 /$

// Based classes:

$/ /$ - Action Used to find a source line number from a trajectory

$/ /$ - Control Used to find which line is a test-control such as

$/ /$ if, while, switch, etc.

$/ /$ - DUset DU(Xp) Definition-Use Relation

$/ /$ - IRset Let Xp IR Yt, iff $\mathrm{X}=\mathrm{Y}$ is the identity relation

$/ / \quad$ IR on M(Front $(\mathrm{T}, \mathrm{q}))$.

$/ /$ - TCset Test-Control relation

$1 /$

// Description: A Union set ofDUset, TCset, and IRset.

$1 /$

// Data:

$1 /-2$

$1 /$

$1 /$ Methods :

$/ /$ - Zset(Size) Create DU of size = Size

// Time complexity $O\left(\mathrm{~N}^{\wedge} 2\right)$

$/ /$ - Computez() Compute $\mathrm{Z}$ sets from DUset, IRset, and TCset.

// Time Complexity $O\left(\mathrm{~N}^{\wedge} 2\right)$

$/ /$ - Printz() Print $z$ sets

$1 /$

class Mappair;

template <class T_LineNo, class T_Elm1>

class Action;

template <class T_LineNo, class T_Elm1>

class Control;

template <class T_LineNo, class T_Elm1, class T_Elm2>

class DUset;

template <class T_LineNo, class T_E1m1, class T_Elm2> class IRset;

template <class T_LineNo, class T_E1m1, class T_Elm2>

class Zset : virtual public Action <T_LineNo, T_ElmI>, virtual public Control <T_LineNo, T_Elm1>, public DUset <T_LineNo, T_Elm1, T_Elm2>, public TCset <T_LineNo, T_Elm1, T_Elm2>,

protected: public IRset <T_LineNo, T_Elm1, T_Elm2>

MapPair <T_LineNo, T_Elm1, T_Elm2> $z_{i} \quad / /$ create arrays of $z$ set public:

Zset(const T_LineNo \&Size) : Z(Size), Action(Size), Control(Size),

void Computez (void)

void Printz(void);

;

template < class T_LineNo, class T_Elm1, class T_Elm2>

void Zset< T_LineNo, T_Elm1, T_Elm2>

: : Printz(void) 1

cout $<<"<<<~ Z$ sets $\gg>"<<e n d l$;

\}

Z.PrintSet (Act);

template < class T_LineNo, class T_Elm1, class T_Elm2>

void zset< T_LineNo, T_Elm1, T_Elm2>

: : Computez (void) [

map $<$ T_Elm1, T_Elm2>: : iterator pDU;

map $<T_{-} E 1 \mathrm{~m} 1, T \_E 1 \mathrm{~m} 2>\mathrm{xDU}$;

map<T_Elm1, T_Elm2>: : iterator pIR; // point to IR sets

map<T_Elm1, T_Elm2> xIR; 


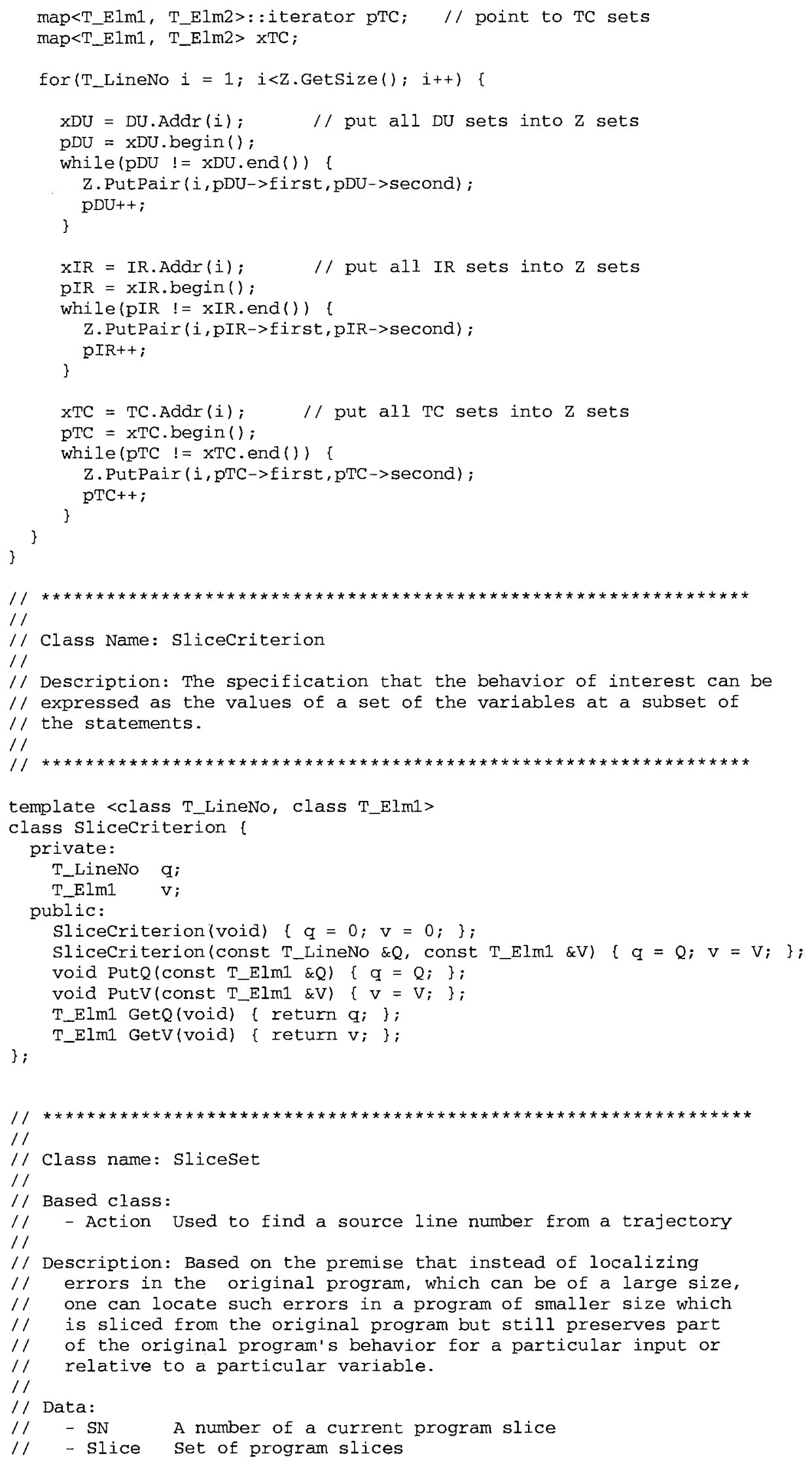


$1 /$

$1 /$ Methods:

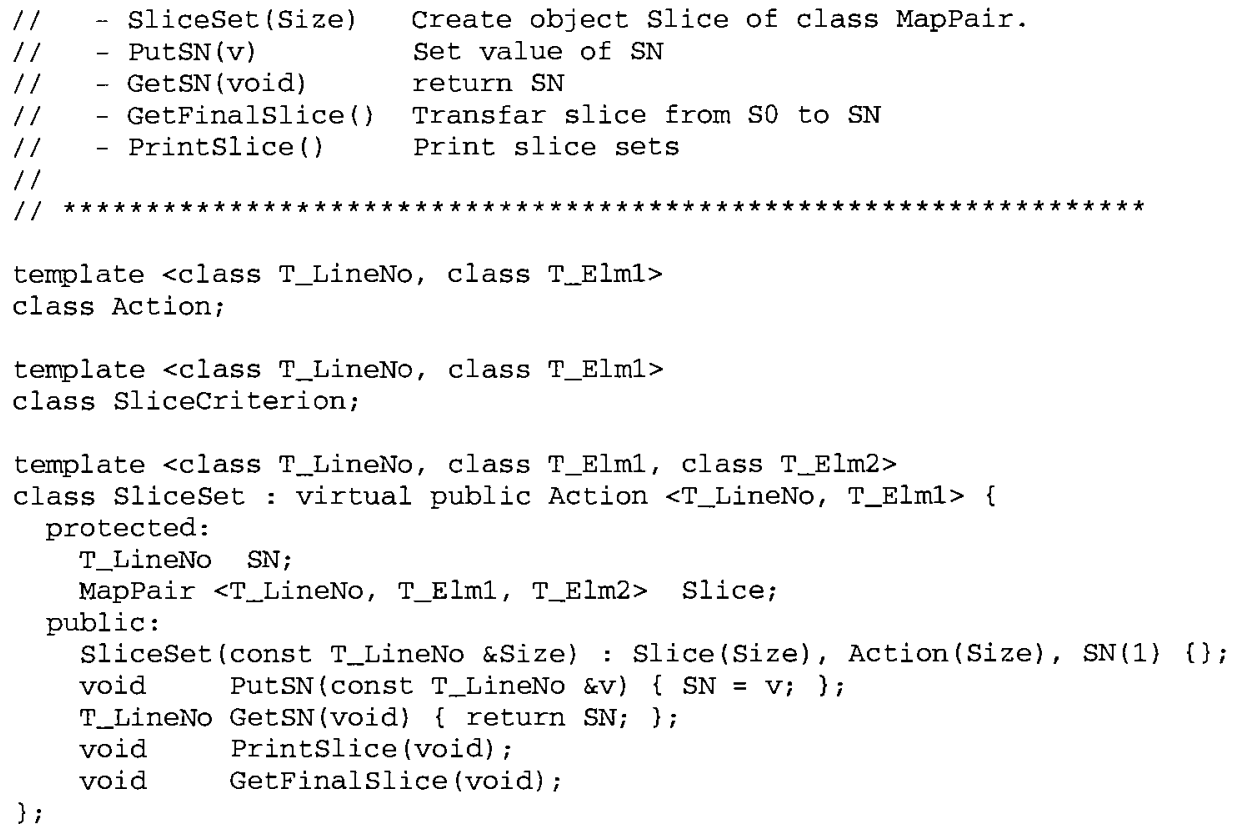




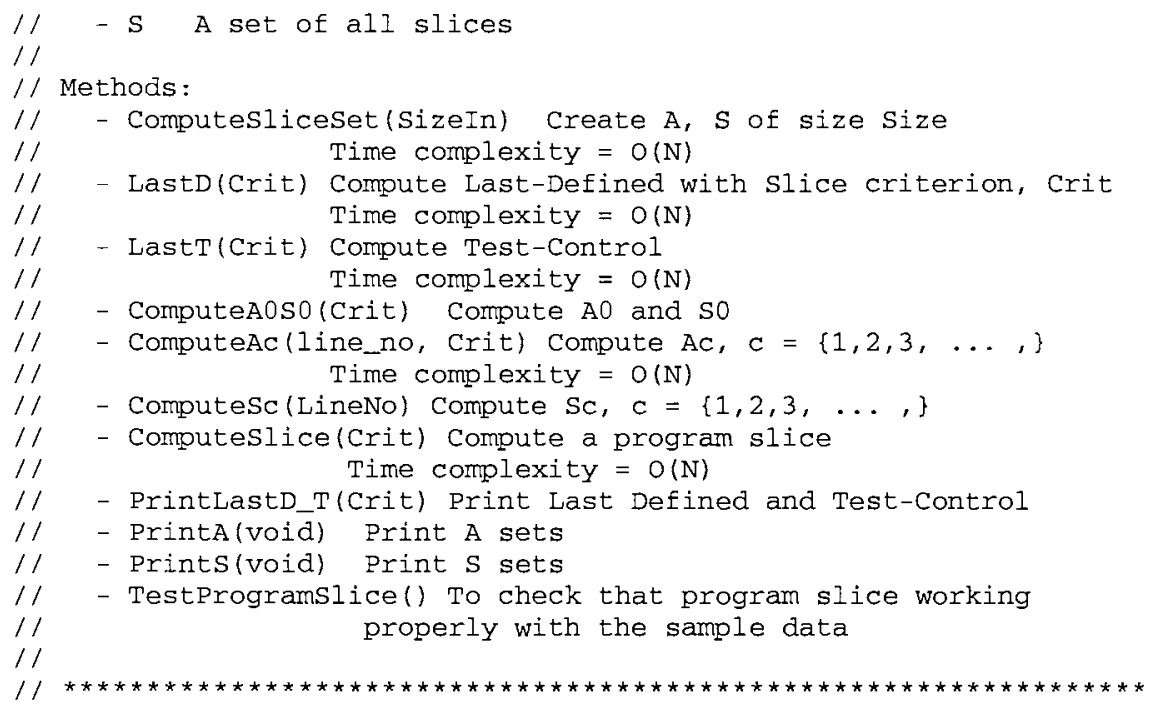

template <class T_LineNo, class T_Elm1>

class Action;

template <class T_LineNo, class T_Elm1>

class Control;

template <class T_LineNo, class T_Elm1, class T_Elm2>

class Zset;

template <class T_LineNo, class T_Elm1, class T_Elm2>

class Sliceset;

template <class T_LineNo, class T_Elm1, class T_Elm2>

class Computesliceset : virtual public Action <T_LineNo, T_Elm1> virtual public Control <T_LineNo, T_Elm1>, public zset <T_LineNo, T_Elm1, T_Elm2>, public Sliceset <T_LineNo, T_Elm1, T_Elm2> \{

private:

MapPair <T_LineNo, T_Elm1, T_E1m2> A; // create arrays of A sets

MapPair <T_LineNo, T_Elm1, T_Elm2> $S ; \quad / /$ create arrays of $S$ sets public:

ComputeSliceSet (const T_LineNo \&SizeIn);

void Printa(void);

void Prints(void);

void PrintLastD $T$ (SliceCriterion $<T$ LineNo, T_Elm1 $>\&$ Crit)

T_Elm2 LastD(SIiceCriterion <T_LineNo, T_Elm1> \&Crit);

T_E1m2 LastT (SliceCriterion <T_LineNo, T_Elm1> \&Crit);

void ComputeAoso(Slicecriterion <T_LineNo, T_Elml> \&Crit);

bool ComputeAc (const T_LineNo \&LineNo, SliceCriterion <T_LineNo, T_Elm2> \&Crit);

void Computesc (const T_LineNo \&LineNo);

void Computeslice(SliceCriterion <T_LineNo, T_Elm1> \&Crit);

\} ;

void TestProgramslice(void);

template < class T_LineNo, class T_Elm1, class T_Elm2>

Computes1iceSet<T_LineNo, T_Elm1, T_Elm2>

: : ComputeSliceSet (const T_LineNo \&Size): A(Size), S(Size),

Action (Size)

Control (Size),

Zset (Size),

Sliceset (Size) \{ \};

template < class T_LineNo, class T_Elm1, class T_Elm2>

void Computesliceset< T_LineNo, T_Elm1, T_Elm2>

: :PrintLastD_T(SliceCriterion <T_LineNo, T_Elm1> \&Crit) \{

cout $<"$ Slice Criterion at $V="<<$ Crit.GetV() $<", Q="<<$ Crit.GetQ ()$<<$ endl $<$ endl;

cout $<<"$ Last Def $=$ "<<LastD(Crit) $<<$ endl; 


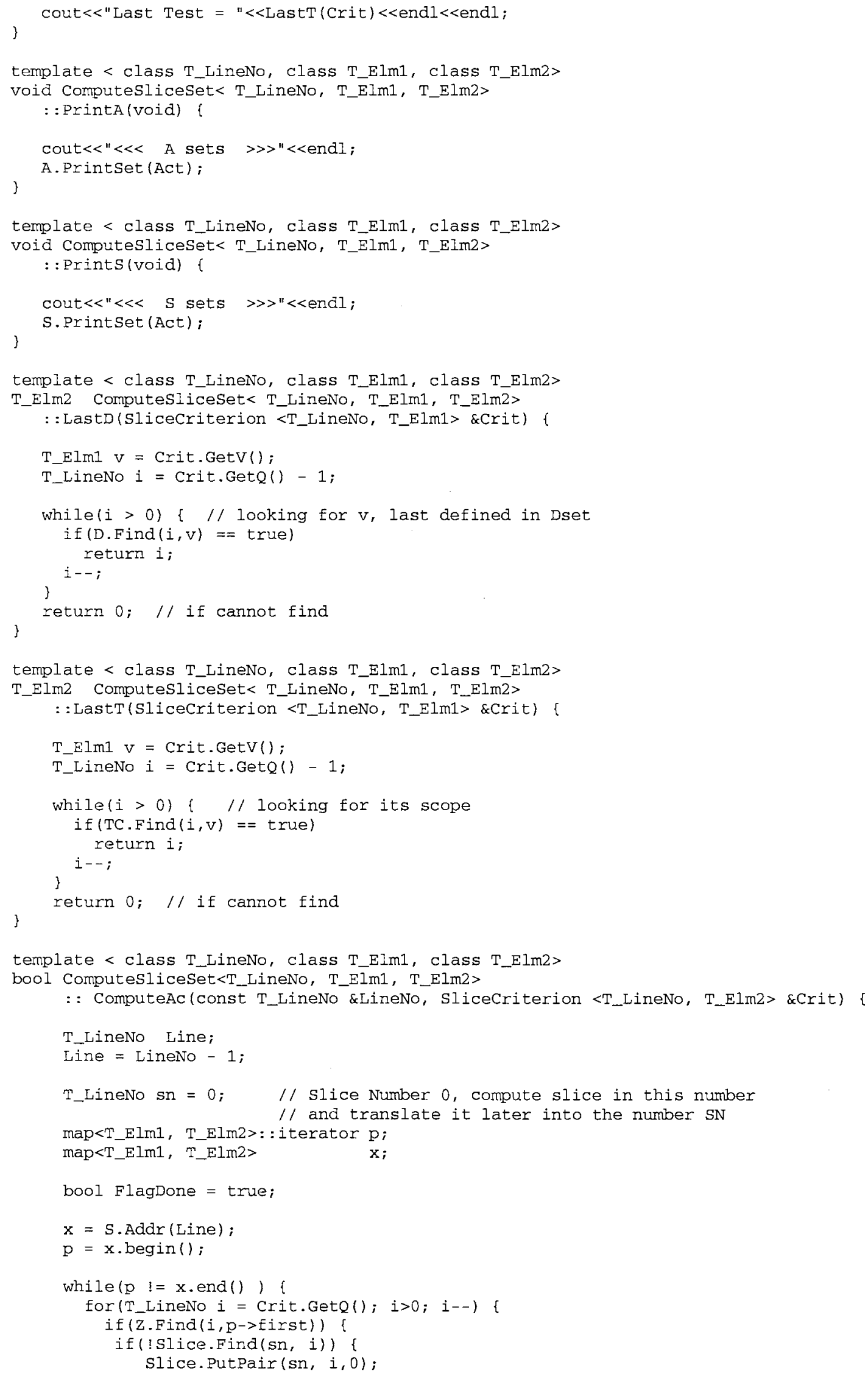




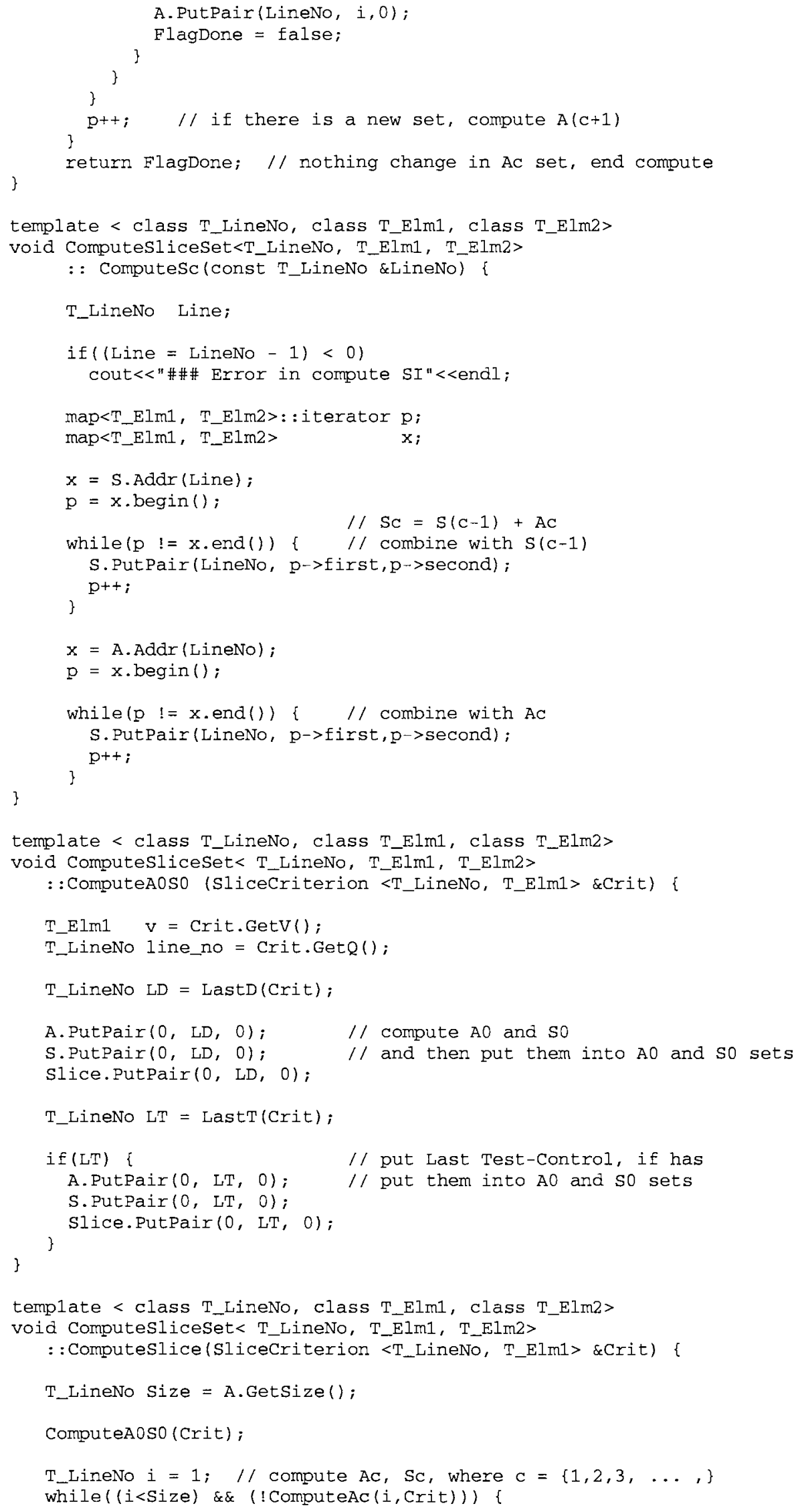




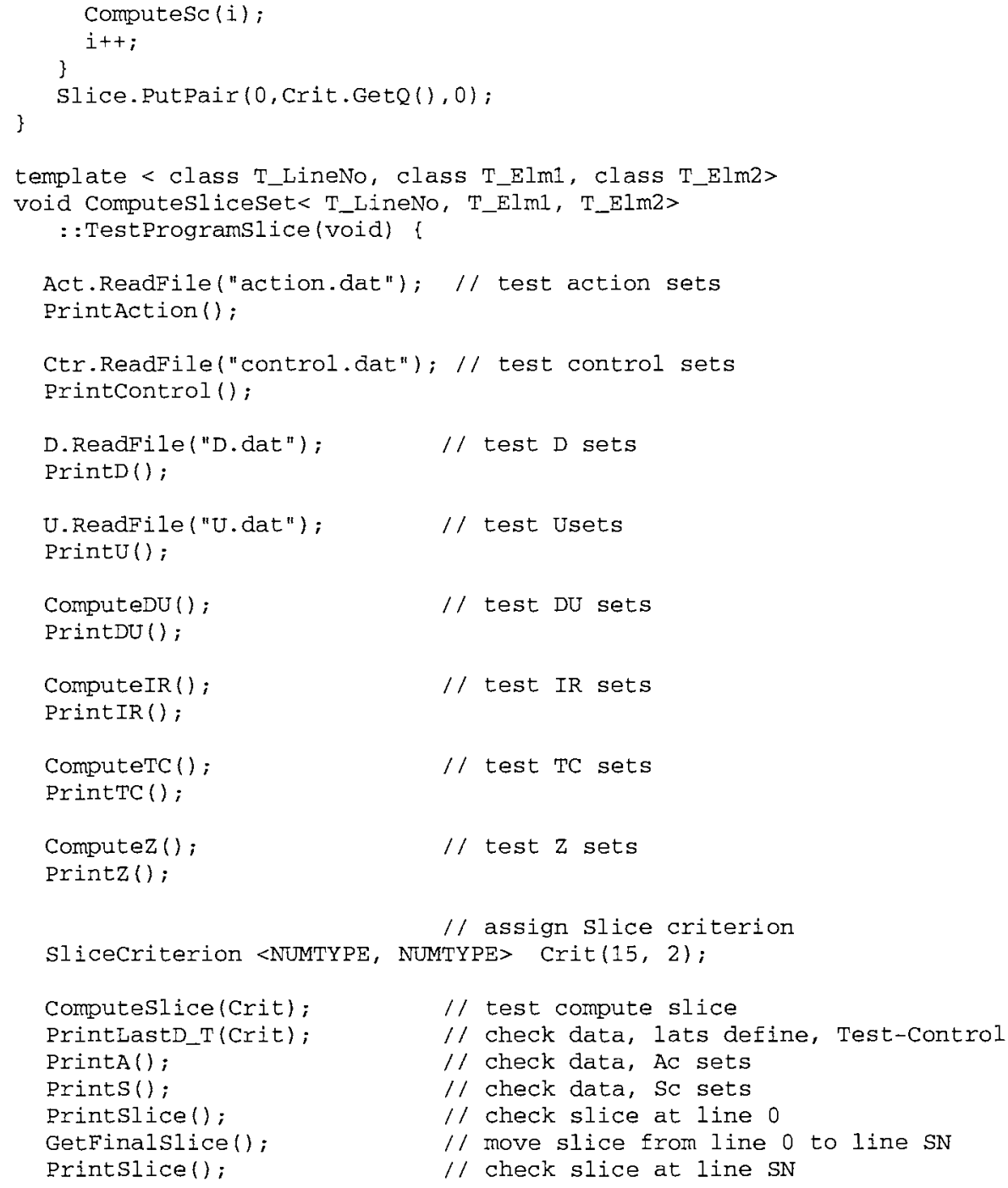




\author{
VITA 2 \\ Winai Wichaipanitch \\ Candidate for the Degree of \\ Doctor of Philosophy
}

\title{
Thesis: AN INTERACTIVE DEBUGGING TOOL FOR C++ BASED ON DYNAMIC SLICING AND DICING
}

\author{
Major Field: Computer Science
}

Biographical:

Personal Data: Born in Lopburi, Thailand, October 23, 1958, the son of Arun and Tonghaw. Married to Cholada Singhasurasakdhi on March 9, 1984.

Education: Graduated from Lopburi Vocational College, Lopburi, Thailand, in May 1977; received the Bachelor of Science in Electrical Engineering degree with a Major in Electronics from the Rajamangala Institute of Technology, Bangkok, Thailand in April 1984; received the Master of Science degree in Computer Science at the Computer Science Department of Oklahoma State University in December 1992; completed the requirements for the Doctor of Philosophy degree in Computer Science at the Computer Science Department of Oklahoma State University in August 2003.

Professional Experience: Instructor, Department of Electrical Engineering, Rajamangala Institute of Technology, 1979 to present. 